\title{
VAPOR GROWTH OF MERCURIC IODIDE TETRAGONAL PRISMATIC CRYSTALS \\ by
}

\author{
ELSA ARIESANTI
}

B.S., University of Virginia, 1999

M.S., University of Michigan, 2001

\section{AN ABSTRACT OF A DISSERTATION \\ submitted in partial fulfillment of the requirements for the degree DOCTOR OF PHILOSOPHY}

Department of Mechanical and Nuclear Engineering

College of Engineering

KANSAS STATE UNIVERSITY

Manhattan, Kansas

2011 


\section{ABSTRACT}

The effect of polyethylene addition on the growth of mercuric iodide $\left(\mathrm{HgI}_{2}\right)$ tetragonal prismatic crystals is examined. Three types of polyethylene powder are utilized: low molecular weight $\left(\overline{\mathrm{M}}_{\mathrm{w}} \sim 4 \times 10^{3}\right)$, ultra high molecular weight $\left(\overline{\mathrm{M}}_{\mathrm{w}} \sim 3-6 \times 10^{6}\right)$, and spectrophotometric grade polyethylenes. Among these types of polyethylene, the low molecular weight polyethylene produces the most significant change in $\mathrm{HgI}_{2}$ morphology, with $\{110\}$ being the most prominent crystal faces. Thermal desorption - gas chromatography/mass spectroscopy (TD-GC/MS) studies show that thermal desorption of the low molecular weight polyethylene at $100^{\circ} \mathrm{C}$ and $150^{\circ} \mathrm{C}$ produce isomers of alkynes, odd nalkanes, and methyl (even-n) alkyl ketones. $\mathrm{HgI}_{2}$ growth runs with n-alkanes, with either neicosane, $\mathrm{n}$-tetracosane, or n-hexatriacontane, cannot replicate the crystal shapes produced during growth with the low molecular weight polyethylene, whereas $\mathrm{HgI}_{2}$ growth runs with ketones, with either 3-hexadecanone or 14-heptacosanone, produce $\mathrm{HgI}_{2}$ tetragonal prismatic crystals, similar to the crystals grown with the low molecular weight polyethylene. C-O double bond contained in any ketone is a polar bond and this polar bond may be attracted to the mercury atoms on the top-most layer of the $\{110\}$ faces through dipoledipole interaction. As a result, the growth of the $\{110\}$ faces is impeded, with the crystals elongated in the [001] direction and bounded by the $\{001\}$ faces along with large, prismatic $\{110\}$ faces. 


\title{
VAPOR GROWTH OF MERCURIC IODIDE TETRAGONAL PRISMATIC CRYSTALS \\ by
}

Elsa Ariesanti

B.S., University of Virginia, 1999

M.S., University of Michigan, 2001

\author{
A DISSERTATION \\ submitted in partial fulfillment of the \\ requirements for the degree \\ DOCTOR OF PHILOSOPHY \\ Department of Mechanical and Nuclear Engineering \\ College of Engineering \\ KANSAS STATE UNIVERSITY \\ Manhattan, Kansas \\ 2011
}

Approved by:

Major Professor

Douglas McGregor 


\title{
Copyright
}

\author{
Elsa Ariesanti
}

2011 


\section{TABLE OF CONTENTS}

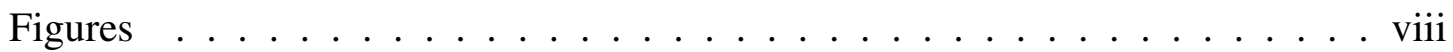

Tables ............................

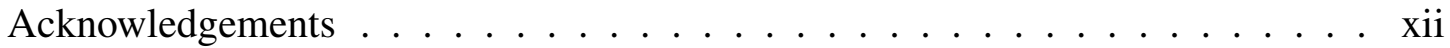

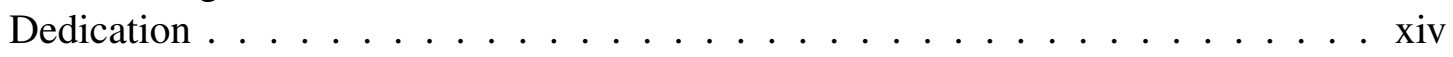

1 Introduction 1

2 Background 5

2.1 Mercuric Iodide Polymorphs . . . . . . . . . . . . . . . . . 5

2.2 Vapor Growth of $\mathrm{HgI}_{2}$ in the Vertical Furnace . . . . . . . . . . . . . . 7

2.3 Vapor Growth of $\mathrm{HgI}_{2}$ in the Horizontal Furnace . . . . . . . . . . . . 10

$2.4 \mathrm{HgI}_{2}$ growth in the SMART Laboratory . . . . . . . . . . . . . . . 13

3 Theory 15

3.1 Crystal structure of $\alpha-\mathrm{HgI}_{2} \ldots \ldots \ldots \ldots \ldots \ldots$

3.2 Prediction of Crystal Morphology . . . . . . . . . . . . . 19

3.2.1 Morphology of Crystal according to the BFDH Rule . . . . . . . . 20

3.3 Morphology of $\mathrm{HgI}_{2} \ldots \ldots \ldots \ldots \ldots \ldots$

3.3 .1 According to BFDH rule . . . . . . . . . . . 22

4 Experimental Methods and Procedures 24

$4.1 \mathrm{HgI}_{2}$ Purification Procedures . . . . . . . . . . . . . . . . . 24

4.1.1 Purification by Sublimation in Dynamic Vacuum . . . . . . . . . . 24

4.1.2 Purification by Melting . . . . . . . . . . . . . . . 26

4.1.3 Purification by Sublimation in Static Vacuum . . . . . . . . . . . 28

4.1.4 Impurity characterization of $\mathrm{HgI}_{2}$ starting material . . . . . . . . . . 29

4.2 Ampoule Preparation and Sealing Procedures . . . . . . . . . . . . . . 30

4.2.1 Ampoule Preparation . . . . . . . . . . . . . . . 31

4.2.2 Ampoule Station . . . . . . . . . . . . . . . . . 32

4.2.3 Sealing Procedures . . . . . . . . . . . . . 33

4.3 Horizontal Furnaces . . . . . . . . . . . . . . . . . . 33

4.4 Growth With No Additive . . . . . . . . . . . . . . . . . . . . . . . . . . . . . . . . 37

4.5 Growth With Polyethylene . . . . . . . . . . . . . . . 37

4.5.1 Pre-growth polyethylene processing step . . . . . . . . . . 38

4.5.2 Growth with the pre-growth polyethylene processing step . . . . . . 39 
4.5.3 Growth without the polyethylene processing step . . . . . . . . 39

4.6 Growth with n-Alkanes . . . . . . . . . . . . . . . . . . 40

4.7 Growth with Ketones . . . . . . . . . . . . . . . . . . . . . . 40

4.8 Material Characterization . . . . . . . . . . . . . . . . 41

4.8.1 TD-GC/MS study polyetylene and n-alkanes . . . . . . . . . . 41

4.8.2 ATR-IR spectroscopy . . . . . . . . . . . . . . . . 42

$4.9 \mathrm{HgI}_{2}$ Frisch Collar Detectors . . . . . . . . . . . . . . . . . 42

4.9.1 Fabrication of $\mathrm{HgI}_{2}$ Frisch collar detectors . . . . . . . . . . . 43

4.9.2 $\gamma$-Spectroscopy with $\mathrm{HgI}_{2}$ Frisch collar detectors . . . . . . . . . . . 45

4.9.3 Charge collection efficiency measurements . . . . . . . . . 45

5 Results and Analysis $\quad 48$

5.1 Purification results and characterization by GDMS . . . . . . . . . . . 48

5.2 Growth With No Additive . . . . . . . . . . . . . . . . . . . . . . . . . . . . . 52

5.2 .1 The effect of source mass . . . . . . . . . . . . 52

5.2.2 The effect of residual pressure . . . . . . . . . . . . 53

5.3 Growth With Pre-Growth Polyethylene Processing . . . . . . . . . . 56

5.3.1 Growth with spectrophotometric grade polyethylene . . . . . . 56

5.3.1.1 Growth results . . . . . . . . . . . . 56

5.3.1.2 Factorial analysis .................. 60

5.3.1.2.1 Normal probability plot ......... 61

5.3.1.2.2 Lenth's plot . . . . . . . . . . . 62

5.3.2 Growth with low $\overline{\mathrm{M}}_{\mathrm{w}}$ polyethylene . . . . . . . . . . . . 63

5.3.3 Growth with ultra high $\overline{\mathrm{M}}_{\mathrm{w}}$ polyethylene . . . . . . . . . . 66

5.3.4 Growth ampoule observations . . . . . . . . . . . . 67

5.3.5 Growth with unmixed $\mathrm{HgI}_{2}$ and polyethylene source . . . . . . . 70

5.4 Growth Without Pre-Growth Polyethylene Processing . . . . . . . . . . 73

5.5 Growth With n-Alkanes . . . . . . . . . . . . . . . . . . 77

5.5.1 Growth with $\mathrm{n}-\mathrm{C}_{20} \mathrm{H}_{42} \ldots \ldots \ldots \ldots 77$

5.5 .2 Growth with $n-\mathrm{C}_{24} \mathrm{H}_{50} \ldots \ldots \ldots \ldots$

5.5 .3 Growth with $n-\mathrm{C}_{36} \mathrm{H}_{74} \ldots \ldots \ldots . \ldots . \ldots$

5.5.4 Summary of growth with alkanes . . . . . . . . . . . 81

5.6 Growth with Ketones . . . . . . . . . . . . . . . . . . . . . . . . . . . 83

5.6.1 Growth with 3-hexadecanone . . . . . . . . . . . . 83

5.6.2 Growth with 14-heptacosanone . . . . . . . . . . . . 85

5.7 Characterization of Polyethylene and n-Alkanes . . . . . . . . . . . 88

5.7.1 TD-GC/MS study of polyetylene and n-alkanes . . . . . . . . 88

5.7.2 ATR-IR spectroscopy . . . . . . . . . . . . . . 93

$5.8 \mathrm{HgI}_{2}$ Frisch Collar Detector . . . . . . . . . . . . . . . . . . 95

5.9 Observations of the Grown Crystals . . . . . . . . . . . . . 103

5.9.1 Growth layer: $\mathrm{HgI}_{2}$ grown without additive . . . . . . . . . . 103

5.9.2 Growth layer: $\mathrm{HgI}_{2}$ grown with low $\overline{\mathrm{M}}_{\mathrm{w}}$ polyethylene . . . . . . . 106

5.9.3 Crystal morphology: computer-generated models . . . . . . . . . 108

5.9.4 Crystal morphology: observations on crystals . . . . . . . . . . . 116

5.9.5 Proposed hypothesis . . . . . . . . . . . . . 126 
6 Conclusions 134

6.1 Future works . . . . . . . . . . . . . . . . 136

6.1.1 Examinations on crystal properties . . . . . . . . . . . 137

6.1.1.1 X-Ray diffraction by Laue back-reflection method . . . . 137

6.1.1.2 Material analysis with photoluminescence . . . . . . . 138

6.1.2 Purity of the starting organic materials . . . . . . . . . . . . 138

6.1.3 Energy consideration for crystal models . . . . . . . . . . . . . 139

A Factorial Analysis $\quad 140$

A.1 $2^{k}$ factorial design . . . . . . . . . . . . . . . . . . . . 140

A.2 Methods of analysis . . . . . . . . . . . . . . . . . 141

A.2.1 Normal probability plot . . . . . . . . . . . . . 141

A.2.2 Lenth's plot . . . . . . . . . . . . . . . . . . . 142 


\section{FIGURES}

1.1 Mass absorption coefficient . . . . . . . . . . . . . . 2

2.1 Graphical representation of the Scholz method . . . . . . . . . . . . 8

$3.1 \mathrm{HgI}_{2}$ unit cell . . . . . . . . . . . . . . . . 16

3.2 Space-group $\mathrm{P}_{2} / n m c \ldots \ldots \ldots \ldots \ldots$

$3.3 \mathrm{HgI}_{2}$ unit cell with angles and distances . . . . . . . . . . . . . . 18

3.4 Crystals with the same habit . . . . . . . . . . . . . . . 19

3.5 Crystals with the same forms . . . . . . . . . . . . . . . 19

$3.6 \mathrm{HgI}_{2}$ crystal morphology according to the $\mathrm{BFDH}$ rule . . . . . . . . . 23

4.1 Experimental Procedures . . . . . . . . . . . . . . . 25

4.2 The equipment used for purifying $\mathrm{HgI}_{2}$ by dynamic sublimation . . . . . . 26

4.3 Purification by Dynamic Sublimation . . . . . . . . . . . . . . 27

4.4 Double chamber ampoule . . . . . . . . . . . . . . . . 27

4.5 A sealed 2-chamber ampoule prior and after the melting procedure . . . . . 28

4.6 A double-chamber ampoule in the static sublimation furnace . . . . . . . . 29

4.7 Double chamber ampoule in static sublimation furnace . . . . . . . . . . 29

4.8 The double chamber ampoule (also seen in Fig. 4.5, after $\mathrm{HgI}_{2}$ sublimation in static vacuum . . . . . . . . . . . . . . . . . 30

4.9 $\mathrm{HgI}_{2}$ retrieval from melting ampoules by sublimation in dynamic vacuum . 30

4.10 Schematics of a growth ampoule and a plug . . . . . . . . . . . . 31

4.11 Ampoule sealing station . . . . . . . . . . . . . . 32

4.12 A sealed ampoule after the ampoule sealing procedure . . . . . . . . 33

4.13 Schematics of a horizontal growth furnace . . . . . . . . . . . . 35

4.14 Horizontal furnaces as set up in Ward Hall room 10A . . . . . . . . . . . . 36

4.15 Types of ketones used in $\mathrm{HgI}_{2}$ growth . . . . . . . . . . . . . . . . . . . . . . 41

$4.16 \mathrm{HgI}_{2}$ Frisch collar detector cross section . . . . . . . . . . . . . . . . . 44

4.17 Setup for spectroscopy measurement using a $\mathrm{HgI}_{2}$ Frisch collar detector . . 46

4.18 The irradiated points on a $\mathrm{HgI}_{2}$ Frisch collar device for the CCE measurement 47

5.1 Retrieval of melted charge in one-chamber melting ampoules by sublimation 49

$5.2 \mathrm{HgI}_{2}$ growth results with no additive, with $80 \mathrm{~g}$ of starting $\mathrm{HgI}_{2}$. . . . . . 52

$5.3 \mathrm{HgI}_{2}$ growth results with no additive, with $40 \mathrm{~g}$ of starting $\mathrm{HgI}_{2}$. . . . . . . 53

$5.4 \quad \mathrm{HgI}_{2}$ growth results with no organic additive and $\mathrm{P}(\mathrm{RT}) \sim 4.2$ Torr . . . . . . 54

5.5 More $\mathrm{HgI}_{2}$ growth results with no additive and $\mathrm{P}(\mathrm{RT}) \sim 4.2$ Torr . . . . . . 55 
5.6 Results of the pre-growth processing stage with spectrophotometric grade polyethylene . . . . . . . . . . . . . . . 56 56

5.7 Growth results with spectrophotometric grade polyethylene . . . . . . . 58

$5.8 \mathrm{HgI}_{2}$ crystals grown with spectrophotometric grade polyethylene . . . . . 59

5.9 Normal probability plot . . . . . . . . . . . . . . . . . . 62

5.10 Lenth's plot . . . . . . . . . . . . . . . . . . . 63

5.11 An ampoule after a pre-growth polyethylene processing step with low $\overline{\mathrm{M}}_{\mathrm{w}}$ polyethylene. . . . . . . . . . . . . . . . . . 64

$5.12 \mathrm{HgI}_{2}$ crystals grown with low $\overline{\mathrm{M}}_{\mathrm{w}}$ polyethylene $\ldots \ldots \ldots \ldots . \ldots 64$

5.13 Growth with low $\overline{\mathrm{M}}_{\mathrm{w}}$ polyethylene . . . . . . . . . . . . . 65

5.14 Results of the pre-growth processing step with ultra high $\bar{M}_{w}$ polyethylene . 66

5.15 Growth with ultra high $\overline{\mathrm{M}}_{\mathrm{w}}$ polyethylene . . . . . . . . . . . . 66

5.16 Details of growth with ultra high $\overline{\mathrm{M}}_{\mathrm{w}}$ polyethylene $\ldots \ldots \ldots$. . . . 67

5.17 Observations on a growth ampoule with low $\overline{\mathrm{M}}_{\mathrm{w}}$ polyethylene $\ldots . .667$

5.18 Details of the droplets of polymer condensation found on the plug . . . . 68

5.19 Vaporization of low polyethylene in the absence of $\mathrm{HgI}_{2} \ldots \ldots$. . . . . 69

5.20 Pre-growth processing for a growth runs with separated or unmixed starting materials . . . . . . . . . . . . . . . . . . 71

5.21 Growth results with separated source materials . . . . . . . . . 72

5.22 Growth without pre-growth processing with 0.02 wt- $\%$ of low $\overline{\mathrm{M}}_{\mathrm{w}}$ polyethylene . . . . . . . . . . . . . . . . . . . . 74

5.23 Growth without pre-growth processing with $0.5 \mathrm{wt}-\%$ of low $\overline{\mathrm{M}}_{\mathrm{w}}$ polyethy-

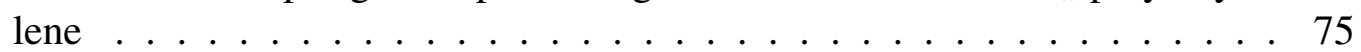

5.24 Growth without pre-growth processing $5 \mathrm{wt}-\%$ of low $\overline{\mathrm{M}}_{\mathrm{w}}$ polyethylene . . 76

5.25 Growth with $\mathrm{n}-\mathrm{C}_{20} \mathrm{H}_{42} \ldots \ldots \ldots \ldots \ldots \ldots$

$5.26 \mathrm{HgI}_{2}$ crystals grown with $\mathrm{n}-\mathrm{C}_{20} \mathrm{H}_{42} \ldots \ldots \ldots \ldots \ldots \ldots \ldots$

5.27 More $\mathrm{HgI}_{2}$ crystals grown with $\mathrm{n}^{-} \mathrm{C}_{20} \mathrm{H}_{42} \quad \ldots \ldots \ldots \ldots$

5.28 Growth with $\mathrm{n}^{-} \mathrm{C}_{24} \mathrm{H}_{50} \ldots \ldots \ldots \ldots \ldots$

5.29 More on growth with $\mathrm{n}-\mathrm{C}_{24} \mathrm{H}_{50} \ldots \ldots \ldots \ldots \ldots \ldots \ldots$

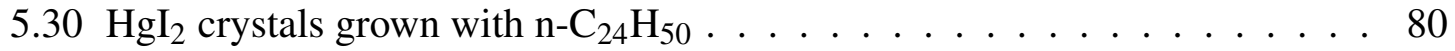

5.31 More on $\mathrm{HgI}_{2}$ crystals grown with $\mathrm{n}-\mathrm{C}_{24} \mathrm{H}_{50} \ldots \ldots \ldots \ldots \ldots$

$5.32 \mathrm{HgI}_{2}$ crystal growth with $\mathrm{n}-\mathrm{C}_{36} \mathrm{H}_{74} \ldots \ldots \ldots \ldots \ldots \ldots$

5.33 More on $\mathrm{HgI}_{2}$ crystal growth with $\mathrm{n}-\mathrm{C}_{36} \mathrm{H}_{74} \ldots \ldots \ldots \ldots . \ldots . \ldots . \ldots 2$

5.34 Growth with 3-hexadecanone $(0.1 \mathrm{wt}-\%) \ldots \ldots \ldots$. . . . . . . 84

5.35 Growth with 14-heptacosanone $(0.1$ wt- $\%) \ldots \ldots \ldots \ldots$

$5.36 \mathrm{HgI}_{2}$ growth with $1 \mathrm{wt}-\%$ 14-heptacosanone . . . . . . . . . . 87

$5.37 \mathrm{Gas}$ chromatograph for thermal desorption of low $\overline{\mathrm{M}}_{\mathrm{w}}$ polyethylene at $100^{\circ} \mathrm{C} 89$

$5.38 \mathrm{Gas}$ chromatograph for thermal desorption of low $\overline{\mathrm{M}}_{\mathrm{w}}$ polyethylene at $150^{\circ} \mathrm{C} 90$

5.39 Gas chromatograph for thermal desorption of ultra high $\overline{\mathrm{M}}_{\mathrm{w}}$ polyethylene . 91

5.40 Gas chromatograph for thermal desorption of n-eicosane $\left(n-\mathrm{C}_{20} \mathrm{H}_{42}\right) \ldots 92$

5.41 Background subtracted ATR-IR spectrum of processed low $\overline{\mathrm{M}}_{\mathrm{w}}$ polyethylene 94

5.42 Background subtracted of ATR-IR spectra of as-grown (110) and cleaved (001) faces of $\mathrm{HgI}_{2}$ crystals . . . . . . . . . . . . . . . . . . . 94

$5.43{ }^{241} \mathrm{Am}$ spectrum collected with a $\mathrm{HgI}_{2}$ Frisch collar detector $\ldots \ldots . .96$

$5.44{ }^{57} \mathrm{Co}$ spectrum collected with a $\mathrm{HgI}_{2}$ Frisch collar detector $\ldots \ldots$. . . 97 
$5.45{ }^{133} \mathrm{Ba}$ spectrum collected with a $\mathrm{HgI}_{2}$ Frisch collar detector . . . . . . . . . 98

5.46 Expanded view of Fig. $5.45 \ldots$. . . . . . . . . . . . . . . . 98

$5.47{ }^{198} \mathrm{Au}$ spectrum collected by a $\mathrm{HgI}_{2}$ Frisch collar detector . . . . . . . . . . 99

$5.48{ }^{137} \mathrm{Cs}$ spectra collected by a $\mathrm{HgI}_{2}$ detector in both planar and Frisch collar configurations . . . . . . . . . . . . . . . . . . . 99

$5.49{ }^{60}$ Co spectrum collected by a $\mathrm{HgI}_{2}$ Frisch collar detector . . . . . . . . . . 100

$5.50{ }^{137} \mathrm{Cs}$ spectra collected by a $\mathrm{HgI}_{2}$ detector in both planar and Frisch collar configurations after being biased for 1,3 , and 8 days . . . . . . . . . . 100

$5.513 \mathrm{D}$ pulse height spectra collected from a collimated ${ }^{137} \mathrm{Cs} \gamma$-ray source along the central line of the detector . . . . . . . . . . . . . 101

5.52 2D pulse height spectra collected from a collimated ${ }^{137} \mathrm{Cs} \gamma$-ray source along the central line of the detector . . . . . . . . . . . . . . . 101

5.53 The CCE profile for the $\mathrm{HgI}_{2}$ Frisch collar detector . . . . . . . . . . . . . 102

$5.54\{110\}$ faces in $\mathrm{HgI}_{2}$ crystal grown in a vertical furnace . . . . . . . . 103

$5.55\{110\}$ faces in $\mathrm{HgI}_{2}$ crystal grown without additive in a horizontal furnace . 104

5.56 Determination of adjacent crystal faces that produce striae on $\{110\}$ in $\mathrm{HgI}_{2}$ grown without additive . . . . . . . . . . . . . . 105

$5.57\{110\}$ faces in $\mathrm{HgI}_{2}$ crystal grown with low $\overline{\mathrm{M}}_{\mathrm{w}}$ polyethylene in a horizontal furnace . . . . . . . . . . . . . . . . 106

5.58 Determination of adjacent crystal faces that produce striae on $\{110\}$ in $\mathrm{HgI}_{2}$ grown with low $\bar{M}_{\mathrm{w}}$ polyethylene . . . . . . . . . . . . . . . . . 107

5.59 Definition of the central distance $r_{(h k l)} \ldots \ldots \ldots$. . . . . . . . . . . . . . . . . . . . . . . . . .

5.60 Notation for crystal face labels in Table 5.8 . . . . . . . . . . . . . 109

5.61 The effect of the ratio between the values of $r_{\{h k l\}}$ in governing crystal shape 111

5.62 Illustration on how $\Delta r_{\{h k l\}}$ may be implied from the presence of $\{\mathrm{hkl}\} \ldots 115$

5.63 Observations on the faces of a crystal in Fig. $5.54 \ldots$. . . . . . . . . 116

5.64 A small single crystal from a growth without any additive in a horizontal

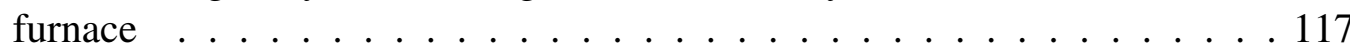

5.65 Another single crystal from a growth without any additive in a horizontal furnace . . . . . . . . . . . . . . . 117

5.66 Crystals in Figs. 5.67 and fig:horzno-twin2 in the growth ampoule . . . . 118

5.67 A twin crystal grown without any additive in a horizontal furnace . . . . . . 118

5.68 Crystal models for the bottom half of the crystal in Fig. 5.67 . . . . . . . 120

5.69 Another twin crystal grown without any additive in a horizontal furnace . . 121

5.70 Single crystals grown with low $\overline{\mathrm{M}}_{\mathrm{w}}$ polyethylene in a horizontal furnace . . 124

5.71 A twin crystal grown with low $\overline{\mathrm{M}}_{\mathrm{w}}$ polyethylene in a horizontal furnace . . 125

5.72 Growth of a (001) plane, $0<c<\frac{1}{2} \ldots \ldots \ldots . \ldots \ldots . \ldots \ldots$

5.73 Growth of a (001) plane, $\frac{1}{2}<c<1 \ldots \ldots \ldots \ldots$. . . . . . . . . . . . . . . . . . . . . . . . . . . .

5.74 Two tetrahedral layers, $0<c<1 \ldots \ldots$. . . . . . . . . . . . . . 129

5.75 Addition of $\mathrm{HgI}^{+}$and $\mathrm{I}^{-}$or $\mathrm{Hg}^{2+}$ and two $\mathrm{I}^{-}$to Fig. 5.72(e) . . . . . . 130

$5.76 \mathrm{Hg}$-I polar covalent bond . . . . . . . . . . . . . . . 130

5.77 Qualitative vector analysis of $\mathrm{Hg}$-I dipoles for a tetrahedral layer in $0<c<\frac{1}{2} 131$

5.78 Qualitative vector analysis of $\mathrm{Hg}$-I dipoles for a tetrahedral layer in $\frac{1}{2}<c<1 \quad 132$

5.79 Combination of Figs. 5.77 and $5.78 \ldots \ldots$. . . . . . . . . . 133 


\section{TABLES}

3.1 Symmetry requirement for $d_{h k l}$ halving for crystal morphology determination 21

$3.2 \mathrm{HgI}_{2}$ growth faces as predicted by the BFDH rule . . . . . . . . 22

$4.1 \mathrm{HgI}_{2}$ samples analyzed by GDMS $\ldots \ldots \ldots \ldots \ldots \ldots$

4.2 Types of polyethylene used in $\mathrm{HgI}_{2}$ growth experiments . . . . . . . . 38

4.3 Variables in factorial analysis of growth with spectrophotometric grade polyethylene . . . . . . . . . . . . . . . . . . 39

4.4 Types of $\mathrm{n}$-alkanes used in $\mathrm{HgI}_{2}$ growth experiments $\ldots \ldots \ldots$

4.5 Types of ketones used in $\mathrm{HgI}_{2}$ growth experiments $\ldots \ldots \ldots \ldots$

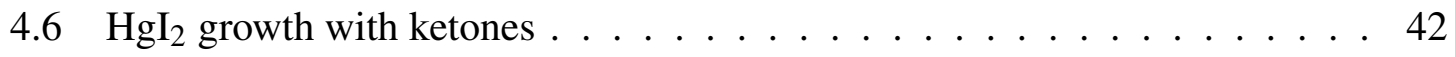

5.1 GDMS results for Group 1 with decreasing impurity concentrations $\ldots . .50$

5.2 GDMS results for Group 1 with increasing impurity concentrations . . . . . 50

5.3 GDMS results for Group $2 \ldots \ldots \ldots \ldots \ldots$

5.4 GDMS results for Group $3 \ldots \ldots \ldots \ldots$

5.5 Factorial analysis of growth with spectrophotometric grade polyethylene . . 57

5.6 The observables and effect calculation . . . . . . . . . . . . . 60

5.7 Probability values and $z$-scores of the effects of growth factors $\ldots \ldots 61$

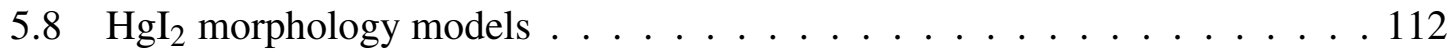

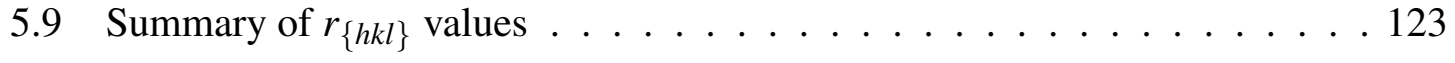

A.1 Yates' algorithm for $2^{3}$ full factorial design $\ldots \ldots \ldots \ldots \ldots$ 


\section{ACKNOWLEDGMENTS}

First and foremost, I thank Jesus Christ, my Lord and Savior, my Life and Joy. All things came to be through Him, and without Him nothing came to be (John 1:3).

I would like to express my gratitute to Dr. Douglas McGregor for being a mentor for all these years. Thank you for teaching me that a good scientist and engineer needs to be a person of integrity. Thank you for your encouragement and patience. Also my sincere thanks to Dr. Kim Kearfott for introducing me to nuclear engineering.

I would also like to thank my committee members, Dr. Christer Aakeröy, Dr. Andrew Rys, and Dr. Ken Shultis, for all your help, comments, and suggestions. Also thanks to my committee chair, Dr. Sanjay Rebello, and a former committee member, Dr. James Edgar, for your comments and suggestions.

This research project would not have been completed without the help from my former and current colleagues in the SMART Laboratory at Kansas State University, as well as those previously at University of Michigan. A special remembrance for the late Holly Gersch, who was taken from us too soon. I am indebted to her, for her example of diligence, humility and tenacity that has inspired me to be a better person and researcher.

My gratitude to former and current administrative staff of the Department of Mechanical and Nuclear Engineering at K-State - Deb Stryker, Erin Carlson, Sarah Buchanan, Sherry Donahey, Carol Konold, and Marcia Chacon - for their help and kindness, and also to Dr. Mo Hosni for his encouragement and kind words.

I would not have been able to pursue a study of $\mathrm{HgI}_{2}$ without the support of funding from the Department of Energy through NEER and DTRA Basic Research (funding no. HDTRA-1-07-1-007) programs. I am grateful for the opportunity afforded by these funds to study a fascinating subject.

Sincere thanks to TN Technologies and Dr. Raj Natarajan for donating the horizontal growth furnaces, and also to MOXTEK for donating the initial starting materials and equipment for growth. 
I am grateful for all the ampoules and glassware created by Jim Hodgson, Master Glassblower at the Department of Chemistry at K-State. Without his expertise, help, and suggestions, those growth runs would not have been possible! I thouroughly enjoyed learning about glassblowing and its intricacies from you.

Special thanks to Drs. Don Nason and Arnold Burger for their comments and suggestions that helped me understand the complexities of growing $\mathrm{HgI}_{2}$.

And finally, my gratitude to my parents, Iwan Trisnawan Santoso and Julianti Boentoro, to my brother, Nico Santoso, and my sister-in-law, Luciana Budiman. Terima kasih atas kasih, doa-doa, dan nasehat. 


\section{Dedication}

To my parents, Iwan Trisnawan Santoso and Julianti Boentoro, and to my brother Nico Santoso,

thank you for your prayers, love and support. 


\section{Chapter 1}

\section{Introduction}

Semiconductor detector technology using single element materials, such as silicon ( $\mathrm{Si}$ ) and germanium (Ge), has been the backbone of the semiconductor detector industry for decades. While the performance of $\mathrm{Si}$ and Ge $\gamma$-ray spectrometers is exceptional, industry needs detectors that exclude cooling. Si and Ge $\gamma$-ray spectrometers are usually cryogenically cooled with liquid nitrogen $\left(\mathrm{LN}_{2}\right)$ to significantly diminish thermal noise. Moreover, $\mathrm{LN}_{2}$ is usually stored in a bulky steel container that can weigh hundreds of pounds when filled. Therefore, most Si or Ge spectrometers commonly are not portable, or, if they are, special arrangements for easy access to $\mathrm{LN}_{2}$ or other types of refrigeration are necessary.

A few other traits besides portability are also sought, such as excellent energy resolution, straightforward material growth, reduction of impurities and defects, and efficient detector production. Accordingly, several compound semiconductors have been proposed and subsequently studied, including cadmium zinc telluride $\left(\mathrm{Cd}_{0.9} \mathrm{Zn}_{0.1} \mathrm{Te}\right.$ or shortened as CdZnTe), mercuric iodide $\left(\mathrm{HgI}_{2}\right)$, and lead iodide $\left(\mathrm{PbI}_{2}\right) . \mathrm{HgI}_{2}$ is considered a candidate for room-temperature $\gamma$-ray detector material, mainly due to its high atomic numbers $\left(\mathrm{Z}_{\mathrm{Hg}}\right.$ $\left.=80, \mathrm{Z}_{\mathrm{I}}=53\right)$ and wide band gap $\left(\mathrm{E}_{\mathrm{g}} \sim 2.13 \mathrm{eV}\right.$ at $\left.300 \mathrm{~K}\right)$. As in Figure 1.1, because of its high $\mathrm{Z}$ constituents, $\mathrm{HgI}_{2}$ has the highest photoelectric absorption coefficient compared to that of $\mathrm{Si}, \mathrm{Ge}$, or $\mathrm{CdZnTe}$, while it is comparable to those of $\mathrm{PbI}_{2}$ and $\mathrm{TlBr}$, for $\gamma$-ray energies in the range of 80 and $1000 \mathrm{keV}$. 


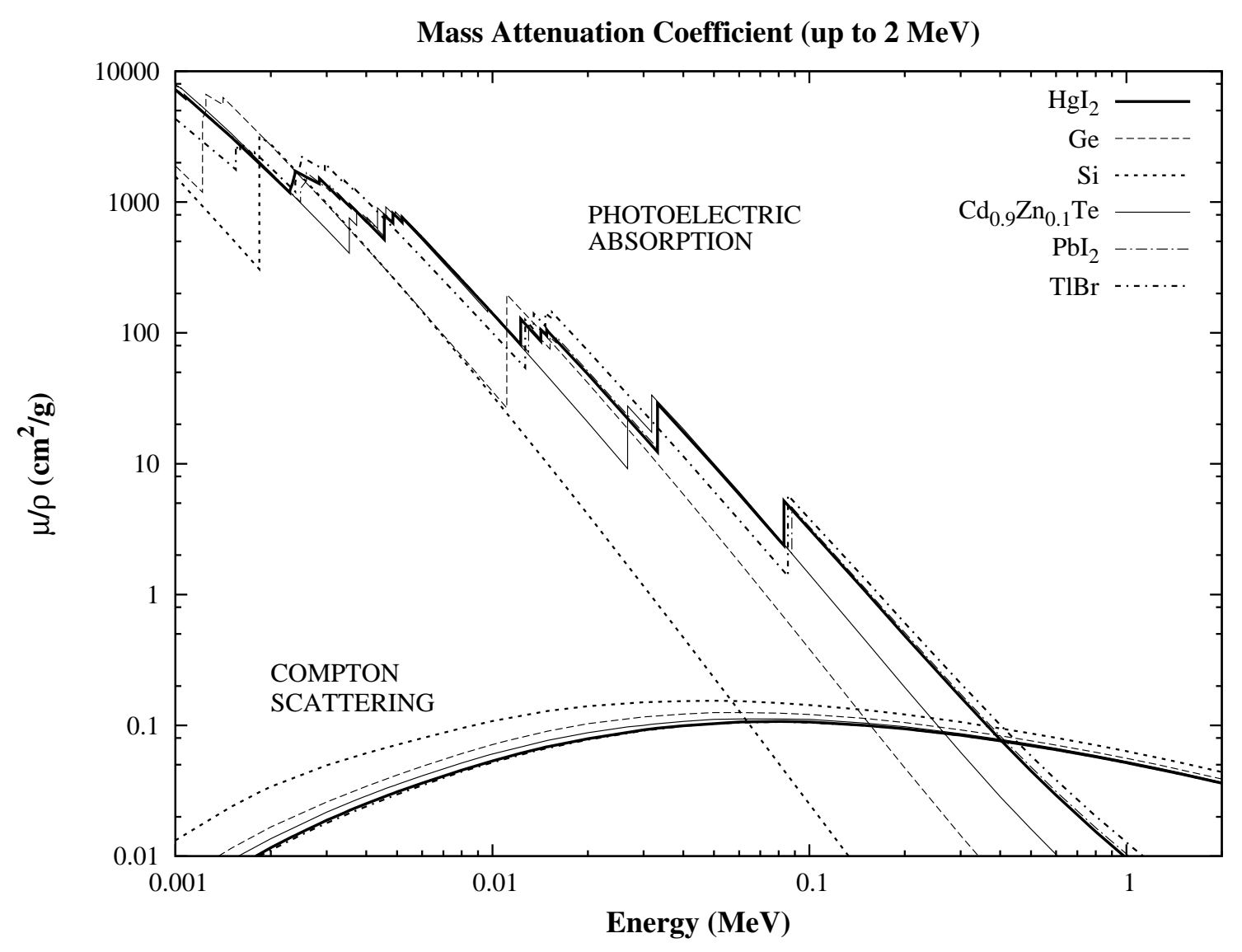

Figure 1.1. Mass absorption coefficients of $\gamma$-ray detector materials. Data were calculated using the online XCOM database [1].

Despite these favorable attributes, $\mathrm{HgI}_{2}$ suffers from several drawbacks, such as low hole mobility [2-4], low yield strength [5-7], and polarization [8-12]. These drawbacks, unfortunately, have prevented a wider use of $\mathrm{HgI}_{2}$ single crystals as room temperature $\gamma$ ray detectors. Charge carriers in $\mathrm{HgI}_{2}$ have relatively low electrical transport properties when compared to other semiconductor materials commonly used for radiation detectors. Similar to these other semiconductors, the hole mobility for $\mathrm{HgI}_{2}$ is lower than the electron mobility. Therefore, the holes will be trapped more easily, which works to reduce the charge collection efficiency (CCE) of the device. Because a large deviation in charge carrier collection translates into poor energy resolution, removal of the hole contribution from the charge induction process and/or the reduction of hole carrier traps will improve energy resolution. 
One design that precludes much of the hole contribution to charge induction is the Frisch collar detector design [13-17]. With this detector design, the device can be designed such that the contribution of the hole motion towards charge induction is inconsequential. In recent years, CdZnTe fabricated Frisch grid detectors have achieved sub-1\% energy resolution, at $662 \mathrm{keV}$, consistently [18]. The simplest Frisch collar detector utilizes a tetragonal prismatic crystal with an aspect ratio of two [19]. Therefore, ordinarily, a bulk crystal must be cut into this same shape. However, cutting a $\mathrm{HgI}_{2}$ crystal into the tetragonal prismatic shape risks damaging the crystal because of its low yield strength. Moreover, subsequently polishing the crystal may introduce additional impurities.

Impurities can also be incorporated through the pre-growth and crystallization processes. Conversely, the appropriate growth method can reduce impurities and diminish the effects of crystal dislocations. First, impurity measurements revealed the inclusion of solution and solvent molecules in $\mathrm{HgI}_{2}$ crystals grown by solution and solvent methods [20-22]. These contaminant molecules included impurities that work to degrade the material properties. Second, growth by melt cannot be employed due to irreversible phase transformation near $130^{\circ} \mathrm{C}$. Third, vapor growth is a more suitable method for growing $\mathrm{HgI}_{2}$, which is known for its appreciable vapor pressure at room temperature. The most common methods of growing $\mathrm{HgI}_{2}$ by vapor are bulk crystal growth in a vertical furnace and polyethylene-aided crystal growth in a horizontal furnace.

The vertical furnace method, developed by EG\&G Santa Barbara [23-25], is based on the modified Scholz method [26]. This method has been used to routinely grow 100-200 gram crystals of $\mathrm{HgI}_{2}$ within a one to two month period. Although much material is harvested, not all parts of the grown crystal are suitable for detector fabrication. Therefore, extensive cutting and etching must be used to shape and prepare the surfaces of the crystals, but these post-growth processes predispose $\mathrm{HgI}_{2}$ to extrinsic impurities and plastic deformation, ultimately threatening crystal integrity. For these reasons, $\mathrm{HgI}_{2}$ crystals cut into various shapes have been fabricated into a Frisch collar detector with limited success [27].

Another promising growth method by vapor for $\mathrm{HgI}_{2}$ is the horizontal method first introduced by Faile in 1980 [28]. This method produces platelet or tetragonal prismatic $\mathrm{HgI}_{2}$ 
crystals in a horizontal furnace in the presence of organic additives. Previous work indicated that $\mathrm{HgI}_{2}$ grows into platelet shaped crystals when unpurified $\mathrm{HgI}_{2}$ was mixed with polyetheylene and subjected to temperatures higher than the $\alpha$-to- $\beta$ - $\mathrm{HgI}_{2}$ phase transformation temperature at $130^{\circ} \mathrm{C}$ [28]. $\mathrm{HgI}_{2}$ grown with purified $\mathrm{HgI}_{2}$ mixed with low $\overline{\mathrm{M}}_{\mathrm{W}}$ polyethylene and subjected to lower temperatures, however, fortuitously yield tetragonal prismatic crystals with adequate dimensions needed to fabricate Frisch collar devices. This advantageous growth feature means that fewer post-growth processes are needed to produce detectors. Unlike the $\mathrm{HgI}_{2}$ vapor growth in a vertical furnace, $\mathrm{HgI}_{2}$ growth with the Faile method has not been fully understood. In the past, efforts to determine the causes for this habit modification focused on the effect of added polymer on mass transport, purification, and $\mathrm{HgI}_{2}$ stoichiometry [29-32]. To date, however, none of these efforts has satisfactorily explained the effect of polymer addition in modifying $\mathrm{HgI}_{2}$ crystal habit.

Previous studies on $\mathrm{HgI}_{2}$ vapor growth by the Faile method are summarized in Chapter 2. Descriptions of the $\alpha-\mathrm{HgI}_{2}$ crystal structure and the Bravais-Friedel-Donnay-Harker law of crystal morphology are explained in Chapter 3. Purification of the $\mathrm{HgI}_{2}$ material and procedures for the growth experiments, completed in the S.M.A.R.T. Laboratory, as well as $\mathrm{HgI}_{2}$ Frisch collar fabrication and spectrometer chracterization are described in Chapter 4. Growth results and analysis, along with $\mathrm{HgI}_{2}$ spectroscopy and characterization results are summarized in Chapter 5. Conclusions and future works are detailed in Chapter 6. 


\section{Chapter 2}

\section{Background}

Choosing a method for growing a specific material mostly depends on its properties. $\mathrm{HgI}_{2}$, with a destructive phase transformation at about $130^{\circ} \mathrm{C}$, is not an ideal material for melt growth; it is generally grown by either solution or vapor growth methods at temperatures below the transformation point. Although $\mathrm{HgI}_{2}$ solution growth may produce large single crystals, however, because of possible inclusion of solvent into the crystal lattice, detectors fabricated from solution grown $\mathrm{HgI}_{2}$ did not perform as well as detectors fabricated from vapor grown crystals. Therefore, more efforts have been spent on $\mathrm{HgI}_{2}$ vapor growth, which is a very viable growth method for $\mathrm{HgI}_{2}$ due to its high vapor pressure. A review of the literature on $\mathrm{HgI}_{2}$ polymorphs is presented in Section 2.1, while a review of the literature on $\mathrm{HgI}_{2}$ horizontal growth, specifically with the aid of organics, is found in Section 2.3.

\subsection{Mercuric Iodide Polymorphs}

$\mathrm{HgI}_{2}$ is known to have several crystal structures at different temperature and pressure ranges. In vapor phase, $\mathrm{HgI}_{2}$ exists as a linear molecule, which disassociate into I and $\mathrm{Hg}$ or $\mathrm{HgI}$ at temperatures beyond $1000^{\circ} \mathrm{C}$ [33]. A thermodynamic study on the composition of the $\mathrm{HgI}_{2}$ vapor calculated that, between $50^{\circ} \mathrm{C}$ to $175^{\circ} \mathrm{C}$ and between $2.6 \times 10^{-4}$ 
to $2.6 \times 10^{-4}$, decomposition of $\mathrm{HgI}_{2}(\mathrm{~g})$ to $\mathrm{I}_{2}(\mathrm{~g})$ and $\mathrm{Hg}(\mathrm{g})$ was possible but only amount to $1-\%$ of the vapor composition. Therefore, for all intense and purposes, the assumption that $\mathrm{HgI}_{2}(\mathrm{~g})$ transports as molecules or analysis of $\mathrm{HgI}_{2}$ vapor transport with a single component, was acceptable [34].

Several polymorphs of mercuric iodide, usually referred to by their apparent colors of red, orange, and yellow, have been found. Solid crystals of $\mathrm{HgI}_{2}$ are usually referred to by their apparent colors, however, the crystal structure depends on the temperature and pressure ranges. In ambient conditions, $\mathrm{HgI}_{2}$ exists as a red crystal (also known as the $\alpha$-phase), which has a tetragonal crystal structure, where the mercury $(\mathrm{Hg})$ atom forms a tetrahedral coordination with the iodine (I) atom [35, 36] (more information on the crystal structure of $\alpha-\mathrm{HgI}_{2}$ can be found in Section 3). Orange and yellow crystals can also be found at ambient conditions, however, these crystals are metastable, and their colors readily change to red when touched. Three structures of orange $\mathrm{HgI}_{2}$ have been discovered $[37,38]$, with all three are different tetragonal structures, each consists of $\mathrm{Hg}_{4} \mathrm{I}_{10}$ supertetrahedra. The metastable yellow crystal is molecular, with linear I-Hg-I molecules $\left(180.4^{\circ}\right)$ packed into an orthorhombic crystal structure.

The red $\alpha$-phase $\mathrm{HgI}_{2}$ turns yellow (also known as the $\beta$ - $\mathrm{HgI}_{2}$ phase) at about $130^{\circ} \mathrm{C}$ (this temperature was highly dependent on the purity of the $\alpha-\mathrm{HgI}_{2}$ ). This high temperature yellow crystal was initially thought to have the same crystal structure as the metastable yellow crystal. A recent study, however, show that the high temperature yellow crystal actually possesses a monoclinic crystal structure with two bent I-Hg-I molecules $\left(157^{\circ}\right.$ and $163^{\circ}$ ) [37]. According to [39], the transformation from $\alpha$ - to $\beta-\mathrm{HgI}_{2}$ involves surface nucleation, where molecules of red $\mathrm{HgI}_{2}$ sublime and yellow nuclei form on the surface. In-situ high temperature study in [37] suggests that the high temperature yellow crystals were polycrystalline, with individual yellow crystallites deemed larger than the original red crystals. Thus, when the yellow crystal cools down to room temperature, although the color turns to red, the crystal is polycrystalline. Hence, the phase transformation from $\alpha$ to $\beta-\mathrm{HgI}_{2}$ is destructive or irreversible as far as crystalinity is concerned. 


\subsection{Vapor Growth of $\mathbf{H g I}_{2}$ in the Vertical Furnace}

The properties of $\mathrm{HgI}_{2}$ allow the material to be grown by the solution method, and although large single crystals of $\mathrm{HgI}_{2}$ have been grown by this method, detectors fabricated from these crytals performed significantly worse than those fabricated from vapor grown crystals. Inclusion of solvent molecules and impurities in the crystal structure were listed as possible causes. Because of this reason, detector-grade $\mathrm{HgI}_{2}$ crystals were usually grown by vapor.

The most frequently employed method to grow large single crystals of $\mathrm{HgI}_{2}$ from its vapor is the vertical (furnace) growth method. The current $\mathrm{HgI}_{2}$ vertical growth method is based on the temperature gradient reversal method, first employed by Scholz [26], who sucessfully grew a large $\mathrm{HgI}_{2}$ crystal. In this method, the growth rate is assumed to be linearly dependent on the difference between source temperature $\left(T_{S}\right)$ and the crystal surface temperature $\left(T_{C}\right)$. The source temperature is varied between two values, $T_{S}^{\min }$ and $T_{S}^{\max }$, and $T_{C}$ is chosen such that

$$
T_{C}=\frac{T_{S}^{\max }+T_{S}^{\min }}{2}
$$

and thus, $T_{C}-T_{S}^{\min }=T_{S}^{\max }-T_{C}$. When the source temperature is at $T_{S}^{\max }$, growth units are transported from the source to the surface of the crystal, within a growth time $\Delta \tau_{\max }$. At $T_{S}^{\min }$, the transport direction of the growth units is reversed, within an etching or evaporation time $\Delta \tau_{\text {min }}$. The sum of the growth and etching times is called the crystallization time $\tau$, and the difference is called the delay time.

The crystallization process is graphically presented in Fig. 2.1. How much a crystal grows is the area $A^{\max }$ under the curve within $\Delta \tau_{\max }$, while how much a crystal etches is the area $A^{\text {min }}$ under the curve within $\Delta \tau_{\text {min }}$.

The ratio $\frac{A^{\min }}{A^{\max }}$ is equal to $\frac{\Delta \tau_{\min }}{\Delta \tau_{\max }}$, which is called the time ratio. The overall growth occurs when $\frac{\Delta \tau_{\min }}{\Delta \tau_{\max }}<1$, while the overall etching occurs when $\frac{\Delta \tau_{\min }}{\Delta \tau_{\max }}>1$. The general condition for growth, therefore, can be written as 


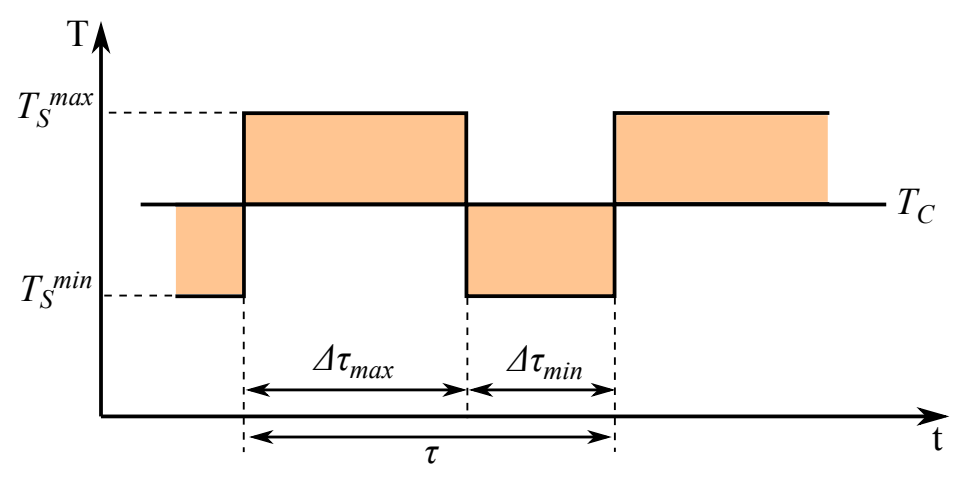

Figure 2.1. Graphical representation of the Scholz method.

$$
\frac{1}{\tau} \int_{0}^{\tau} T_{C}(t) d t<\int_{0}^{\tau} T_{S}(t) d t
$$

with nucleations occur where $\left|T_{C}-T_{S}\right|$ is the largest.

The purpose of the temperature gradient reversal method is nucleating a single crystallite while supressing parasitic nucleations, and these are achieved by careful control of the temperatures, $T_{S}$ and $T_{C}$, as well as the growth and etching times, $\Delta \tau^{\max }$ and $\Delta \tau^{\min }$. Nucleation of a single seed at a specific location is further controlled by adding a heat sink at that location by way of a cold finger, which can either be a thermal conductive metal rod or a stream of sub-room temperature air or gas. Therefore, the temperature gradient between the cold finger, $T_{C F}$, and $T_{C}$ is another constraint in the system. The process is a success, when, by reversal of the temperature gradients, a single crystal nucleates and subsequently grows.

The temperature oscillating method (TOM), which is a modification to the temperature gradient reversal method, besides oscillating the temperature gradient by varying $T_{S}$ between a minimum and a maximum values (a procedure called periodic oscillating source temperature or POST), also employ temperature gradient oscillation by varying $T_{C}$ (periodic oscillating crystal temperature or POCT) [24]. POCT is preferred during the nucleation stage, because the effect of changing $T_{C}$ is more easily observed at shorter $\Delta \tau_{g}$ or $\Delta \tau_{e}$, while POST is preferred during the growth stage, because the growing crystal is held at a 
constant temperature.

The original and modified temperature gradient reversal growth procedures both produce large single crystals of $\mathrm{HgI}_{2}$, however, growth striations occured due to temperature swings. Because growth striations constitute a type of crystal defects, the temperature gradient reversal method has been largely discontinued. The growth furnaces, however, can still be used to grow bulk single crystals, without using of the programmed growth-etching cycles as crystal growth progresses. These cycles, usually not strictly constrained or programmed as in the original or modified procedure, are employed only to nucleate a single crystallite and to avoid parasitic growth of other crystallites at this nucleation stage. The subsequent crystal growth then proceeds by increasing the apparent undercooling, which is $T_{S}-T_{C F}$, by either keeping $T_{S}$ constant while decreasing $T_{C F}$ [40], or keeping $T_{C F}$ constant while increasing $T_{S}$.

As can be gathered from the information above, a close control of temperature and thermal stability is paramount in a succesful nucleation and subsequent growth of a single crystal with the vertical growth method. Variations in thermal settings exist for different pairs of growth ampoule - furnace, thus every growth run has its own individualized settings. Because of parasitic growth of crystallites likely occur during nucleation, a tight temperature control is especially needed in this growth stage. As the growth progresses, morphological stability may become an issue and an overgrowth (a defect) on the crystal surface can happen [40]. Although growth striations caused by temperature swings in the temperature gradient reversal method have been avoided, nevertheless, striations caused by thermal instability are still encountered [24].

The yield of a large single crystal is advantageous, in terms of the large amount of material available for device production. This advantage, however, also comes with several unfortunate consequences. First, because of imperfections or crystal defects, not all parts of the crystal can be utilized, and therefore, the crystal must be cut. Second, $\mathrm{HgI}_{2}$ crystal shapes at the end of growth runs may not be suitable, hence, cutting and shaping the crystal are necessary steps. Because $\mathrm{HgI}_{2}$ has a low yield strength, however, cutting and shaping tend to damage the crystal. Third, along with cutting and shaping, surface polishing is 
also done to render a crystal surface ready for contact deposition. This process, however, exposes the crystal to impurity inclusions.

The second most utilized vapor growth method for $\mathrm{HgI}_{2}$, the growth aided by organics in a horizontal furnace, yields crystals of ideal sizes and shapes to be fabricated directly into devices with no or little post-growth processing [28, 29, 32, 41-46]. The preclusion of much post-growth processing and much shorter crystallization period are the two principal benefits of growing $\mathrm{HgI}_{2}$ by the horizontal method over the vertical method. A considerable amount of comprehension is still lacking, however, especially on how the addition of organics affect $\mathrm{HgI}_{2}$. The main studies on $\mathrm{HgI}_{2}$ growth by the horizontal method are discussed in the next section (Section 2.3)

\subsection{Vapor Growth of $\mathrm{HgI}_{2}$ in the Horizontal Furnace}

$\mathrm{HgI}_{2}$ growth in the horizontal furnace, particulary the growth assisted by organic materials, was first reported by by S. P. Faile, with styrene (used as-received) or cut up (polyethylene) bag used as the organic additives, mixed with the starting $\mathrm{HgI}_{2}[28,47]$. A source temperature of $230^{\circ} \mathrm{C}$ was employed, while the condensation region was at room temperature, where platelets grew in the region below the phase transformation point. Purification of the starting $\mathrm{HgI}_{2}$ was not done because the author believed that $\mathrm{HgI}_{2}$ purification was not needed to produce platelet crystals. Although the formation of $\mathrm{HgI}_{2}$ platelet was not known, and was not attempted to be proven, the author assumed that polymer transport, formation, and/or disassociation might be the factors that influenced the platelet formation.

A few issues in this first study need to be pointed out. First, the use of unpurified $\mathrm{HgI}_{2}$ starting material would allow for undue growth variations, because the amount or type of impurities were not known and subsequently removed. Second, the polyethylene used as an additive in this study could not be quantified and its quality unknown, because it was obtained by cutting up a plastic bag. Third, as mentioned in Section 2.1, the growth temperatures of $150-230^{\circ} \mathrm{C}$ were above the onset temperature for $\alpha$ - to $\beta$-phase transformation, meaning that both $\alpha$ - and $\beta-\mathrm{HgI}_{2}$ crystals can be found in the ampoule. Therefore, not all 
of the crytals can be utilized; as mentioned in Section 2.1, a single crystal $\alpha-\mathrm{HgI}_{2}$ when heated beyond the transformation temperature and then cooled to room temperature, turns to polycrystalline. $\beta-\mathrm{HgI}_{2}$ crystals, which form above the transformation temperature, also turn polycrystalline when cooled to room temperature. Because $\mathrm{HgI}_{2}$, targeted as a roomtemperature radiation detector, is preferred in a single crystal state, growing this material below the phase transformation temperature, hence obtaining strictly $\alpha-\mathrm{HgI}_{2}$, is more ideal.

Following the first study by Faile, a number of studies to solve the question surrounding the effects of organic addition on $\mathrm{HgI}_{2}$ platelet growth were attempted by several groups. A repeat of Faile's study was done using unpurified starting $\mathrm{HgI}_{2}$ and polyethylene as an additive [29], where the observation claimed that although the growth yielded platelets, this type of crystal shapes also occurred even without the addition of polyethylene. Because it was assumed that polyethylene disassociated to molecules with $\overline{\mathrm{M}}_{\mathrm{w}}$ about 700 , the interpretation was that polyethylene did not aid in physical vapor transport, but rather aided chemically in vapor transport. The explanation on the aiding process of the polyethylene in the chemical transport, however, was not given.

The study also looked on the effect of polyethylene addition on the stoichiometry of the grown platelets, as well as the onset temperature for $\alpha-\beta$ transformation. It was noticed that the crystals grown without polyethylene were always mercury rich, and a yelow residue, later identified as mercurous iodide $\left(\mathrm{Hg}_{2} \mathrm{I}_{2}\right)$, was observed in the ampoule where the starting material was placed. It is known, however, that $\mathrm{Hg}_{2} \mathrm{I}_{2}$ is an impurity of $\mathrm{HgI}_{2}$, and that it can be removed by repeated sublimation, either in dynamic or static vacuum (Section 4.1). The study on the onset temperature for $\alpha-\beta$ transformation observed that platelets grown with polyethylene had a slightly higher transformation temperature and more stoichiometric than platelets grown without polyethylene. Open problems identified by this study's author included participation of polyethylene in vapor transport and morphology control by polyethylene. Another study by the same group observed that when the starting material was not purified, the crystal morphology tended towards platelet formation, and when purified $\mathrm{HgI}_{2}$ was used, prismatic crystal habit was obtained. Thus, the study concluded that polyethylene played a role in purifying the starting material by 
separating impurities (for example, $\mathrm{Hg}_{2} \mathrm{I}_{2}$ ) from $\mathrm{HgI}_{2}$ [30].

Another group looked at the growth of $\mathrm{HgI}_{2}$ (purified) with a number of different types of organics additives [41], which produced various crystal shapes. Though the observation indirectly supported the previous conclusions, that $\mathrm{HgI}_{2}$ stoichiometry control and $\mathrm{HgI}_{2}$ purification were accomplished by the organics, nevertheless, no real analysis on the processes was attempted. The study also noticed that some of the organics decomposed during the growth, whether due to high temperature or mixing with $\mathrm{HgI}_{2}$, causing a variation in partial and total pressures were expected, as indicated by an increase in pressure attributed to the residual gases $[31,41]$. The effects of the stoichiometry and purity of the starting $\mathrm{HgI}_{2}$ were deemed inconsequential, unlike claimed previously in [29, 30].

The mass transport calculations determined that $\mathrm{HgI}_{2}$ growth with or without additives were diffusion-limited [31], with a decrease in mass transport or flux attributed to convection $[31,32,48]$. The analysis of the results implies that instead of having the characteristics of a chemical vapor transport, as claimed in [29], the analyzed vapor transport has the characteristics of a physical vapor transport, with sublimation and condensation of $\mathrm{HgI}_{2}$ being the principal crystallization processes [32]. Therefore, the organic materials seemed to behave as inert gases, prompting futher studies in $\mathrm{HgI}_{2}$ mass transport in horizontal furnaces [48-51], as well as $\mathrm{HgI}_{2}$ with inert gases [52, 53]

The presence of residual gases mentioned above and the total pressure inside the ampoule are partially responsible for the shapes of the crystals. The existence of convective flow (causing non-stable growth forms) was implied by the occurrence of needleand dendrite-shaped crystals, while a more stable growth would produce crystallites and platelets. The platelets were obtained when the total pressure inside the ampoule ranged between 2 and 10 Torr [48]. It is worth noting that although the effects of surface processes were recognized [32], however, attempts to explain the surface phenomena were not pursued. 


\section{4 $\quad \mathrm{HgI}_{2}$ growth in the SMART Laboratory}

The $\mathrm{HgI}_{2}$ horizontal growth research in the Semiconductor Materials and Radiation Technologies (SMART) Laboratory at Kansas State University was started based on the prevalent understanding that vapor transport, with factors such as temperature profiles and starting material amounts, had the biggest influence on the growth [53]. For one type of additives, the amount of starting materials and the total pressure in the ampoule did distinguish between stable (denoted by growth of crystallites, tetragonal prismatic crystals, and platelets) and unstable (denoted by growth of needles and dendrites) crystal growth. However, the change in morphology from crystallites, which grow without any additive, to tetragonal prismatic and platelet crystals, both of which grow with certain organics [45, 54], cannot be explained on vapor transport alone.

The focus was thus redirected towards understanding possible crystal surface effects that enabled the formation of tetragonal prismatic crystals during growth with different organics. It is known that impurities (generally speaking, these are ions, atoms, or molecules other than the compounds that constitute the growth units of the crystallizing material) affect growth rates and also growth shapes [55]. Solution growth of $\mathrm{HgI}_{2}$ also show that ions or molecules other than $\mathrm{HgI}_{2}$ did have an effect on the growing crytal surface, hence, on the eventual crystal morphology [20-22].

Therefore, the first objective of the $\mathrm{HgI}_{2}$ research in the Semiconductor Materials and Radiation Technologies (SMART) Laboratory at Kansas State University (KSU) is to determine the effects of organic additives, especially polyethylene, utilized during the horizontal growth of $\mathrm{HgI}_{2}$ on $\mathrm{HgI}_{2}$ crystal morphology. The second objective is to determine an alternative compound that can produce $\mathrm{HgI}_{2}$ tetragonal prismatic crystals in a more predictable

manner. The third objective is to test the suitability of the crystals grown with polyethylene as $\gamma$-ray detectors by fabricating a few crystals as Frisch collar detectors.

Polyethylene was chosen as the primary organic additive, because it was the most often used additive in the horizontal growth of $\mathrm{HgI}_{2}$ by the Faile method. Recognizing that vapor transport depends on molecular weight, also with the assumption that polyethylene vapor- 
izes, different molecular weights of polyethylene were utilized [53,56]. Growth with alkanes and ketones was later pursued, because analysis of the starting materials revealed that these organics were present during $\mathrm{HgI}_{2}$ growth with polyethylene [54]. Crystals grown with alkanes and ketones were qualitatively compared with crystals grown by polyethylene, to determine whether either type of chemicals was a more suitable replacement for polyethylene.

The results and analysis on growth with different types of polyethylene can be found in Sections 5.3 to 5.4, while the growth results with alkanes and ketones can be found in Sections 5.5 and 5.6, respectively. Observations on surfaces and shapes of crystals grown by the horizontal method under various conditions can be found in Sections 5.9.1 to 5.9.4. The effects of adding different types of polyethylene, alkanes, and ketones were judgen on the appearance or disappearance of certain $\mathrm{HgI}_{2}$ crystal faces. The results of a $\mathrm{HgI}_{2}$ Frisch collar detector can be found in Section 5.8. Finally, the proposed hypothesis on the crystal surface phenomena can be found in Section 5.9.5. 


\section{Chapter 3}

\section{Theory}

In ambient growth conditions, at temperatures below $130^{\circ} \mathrm{C}$, the $\mathrm{HgI}_{2}$ crystal forms into a tetragonal crystal structure ( $\alpha$-phase), whereas at temperatures above $130^{\circ} \mathrm{C}$, the crystal forms into a monoclinic crystal structure ( $\beta$-phase). It is the $\alpha$-phase that is of interest for radiation detectors. A review of the $\alpha-\mathrm{HgI}_{2}$ crystal structure is provided in Section 3.1. A method to determine crystal morphology is summarized in Section 3.2, while $\mathrm{HgI}_{2}$ morphology prediction, based on the method, is described in Section 3.3.

\subsection{Crystal structure of $\alpha-\mathrm{HgI}_{2}$}

The $\alpha-\mathrm{HgI}_{2}$ crystal structure, tetragonal with a primitive centering, is stable at room temperature and has a space group designation of $\mathrm{P}_{2} / n m c$, with crystal dimensions of $a=$ $b=4.361 \AA$ and $c=12.450 \AA$ [36]. Fig. 3.1 shows the unit cell, which contains two $\mathrm{HgI}_{2}$ molecules, with two mercury ions at $\left(\frac{1}{4}, \frac{3}{4}, \frac{1}{4}\right)$ and $\left(\frac{3}{4}, \frac{1}{4}, \frac{3}{4}\right)$ and four iodine ions at $\left(\frac{1}{4}, \frac{1}{4}, u\right)$, $\left(\frac{3}{4}, \frac{3}{4}, \frac{1}{2}-u\right),\left(\frac{3}{4}, \frac{3}{4}, \frac{1}{2}+u\right)$, and $\left(\frac{1}{4}, \frac{1}{4}, 1-u\right)$, where $u=0.1107 c$ [36]. The center of symmetry (i.e., inversion center) of the unit cell in Fig. 3.1 is at (000), using the origin choice number 2 described in the International Tables for Crystallography [57]. The $c$-plane projection of the space-group $\mathrm{P} 4_{2} / n m c$ with the origin choice number 2 , as well as symmetry symbols 
and their definitions, are found in Fig. 3.2. ${ }^{1}$

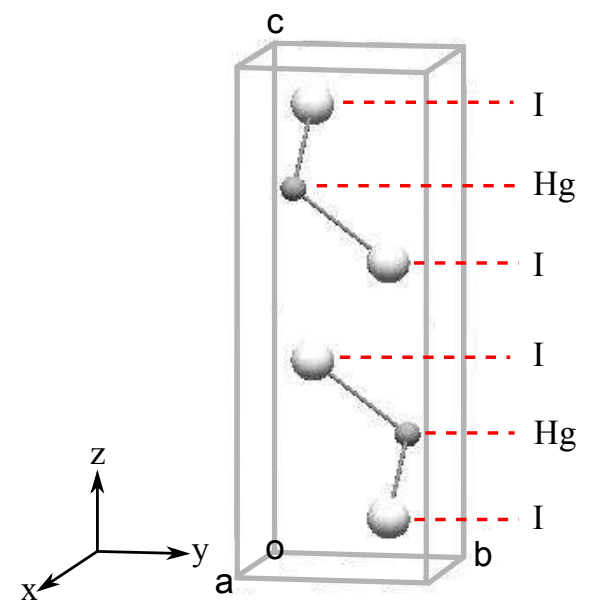

Figure 3.1. $\alpha-\mathrm{HgI}_{2}$ crystal structure containing two $\mathrm{HgI}_{2}$ molecules. The structure was created using DRAWxtl [58].
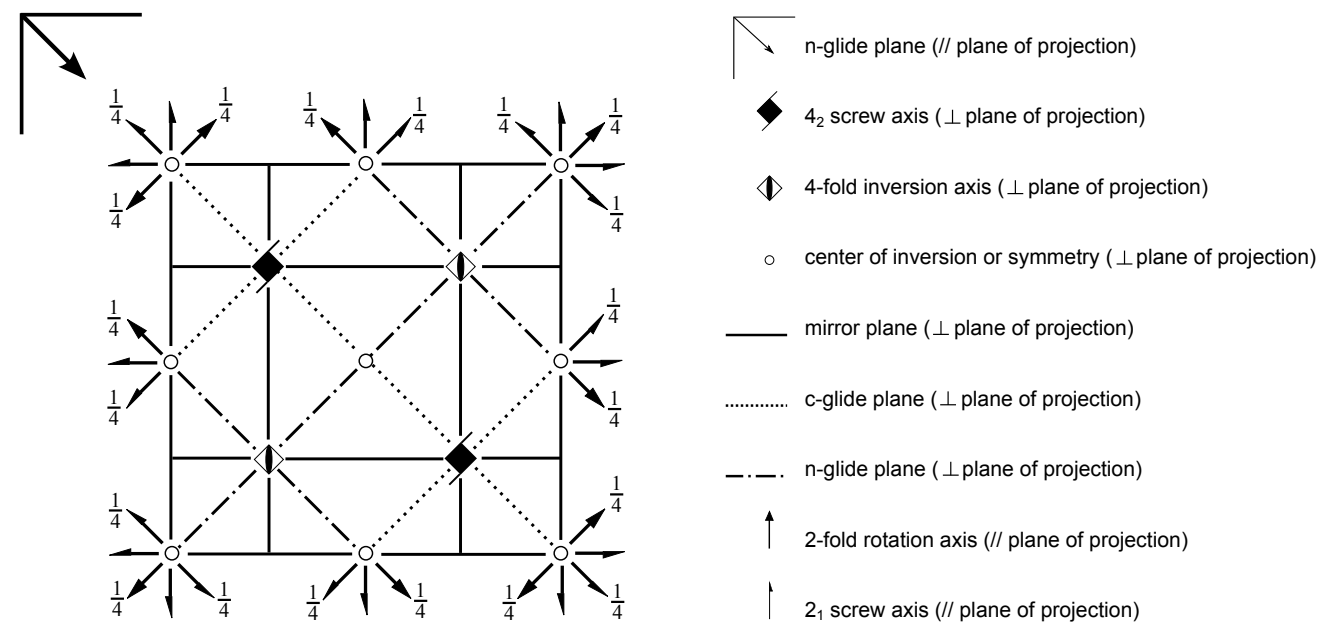

Figure 3.2. Space-group $\mathrm{P} 4_{2} / n m c$ with the origin choice 2 (reproduced from the International Tables for Crystallography [57]).

In $\alpha-\mathrm{HgI}_{2}$ crystal, a mercury ion forms covalent bonds with four iodine bonds in the first coordination sphere in tetrahedral coordination. $\mathrm{HgI}_{2}$ exists as a linear molecule when it is in the vapor state at the practical range of temperatures employed for growth (between

\footnotetext{
${ }^{1}$ The longer Hermann Mauguin notation for this space group is $\mathrm{P} 44_{2} / n 2_{1} / m 2 / c$. This notation indicates the following: $4_{2} / n$ indicates a $4_{2}$ screw axis is perpendicular to an $n$-glide plane, $2_{1} / m$ indicates a $2_{1}$ screw axis is perpendicular to a mirror plane, and $2 / c$ indicates a 2 -fold rotation axis is perpendicular to a $c$-glide plane.
} 
room temperature and $120^{\circ} \mathrm{C}$ ). When the molecule is incorporated into a $\alpha-\mathrm{HgI}_{2}$ crystal, the $\mathrm{Hg}-\mathrm{I}$ bonds are bent ${ }^{2}$ to accommodate the tetrahedral coordination. The tetrahedral configuration is the stable configuration for mercury and iodine in the $\mathrm{HgI}_{2}$ crystal, with the mercury ion in the center of the tetrahedron and one iodine ion at each tetrahedral corner. Therefore, to complete the tetrahedral configuration, the mercury or iodine ions for the added $\mathrm{HgI}_{2}$ molecules covalently bond with the available iodine or mercury ions in the solid, respectively.

Within a $\mathrm{HgI}_{4}^{-2}$ tetrahedron, the $\mathrm{Hg}$-I bonds are all equal by symmetry, but two $\mathrm{I}-\mathrm{Hg}-\mathrm{I}$ angles, $103.14^{\circ}$ and $112.72^{\circ}$, exist [36], as well as three different I-I distances, as shown in Fig. 3.3(a). Each $\mathrm{HgI}_{2}$ molecule in Fig. 3.3(a) belongs to a different layer, and when the tetrahedral coordination is completed for each $\mathrm{Hg}$ center ion (by addition of two more I ions from neighboring molecules), $\mathrm{HgI}_{4}^{-2}$ tetrahedra, one in each layer as shown in Fig. 3.3(b), are formed. Due to the $4_{2}$ screw axis that goes through $\left(\frac{1}{2}, \frac{1}{2}, 0\right)$ and that is parallel to the [001] direction, the tetrahedron in the second layer is rotated $90^{\circ}$ about the screw axis and is translated into the upper half of the unit cell. The tetrahedral layers along the [001] direction are held together by van der Waals forces.

\footnotetext{
${ }^{2}$ According to [37], the formation of $\mathrm{HgI}_{4}^{-2}$ tetrahedra involves breaking and creating the bonds to accomodate the tetrahedral coordination.
} 


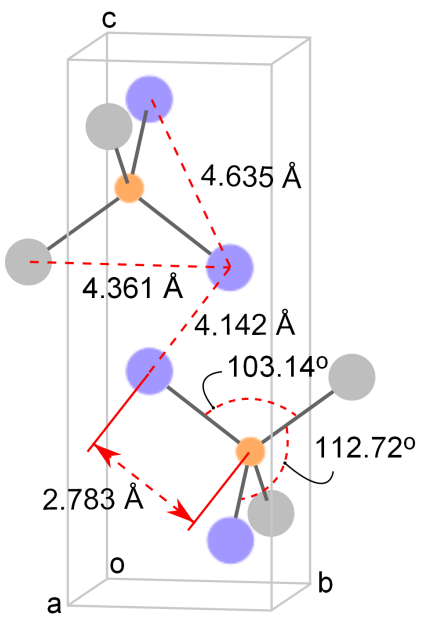

(a)

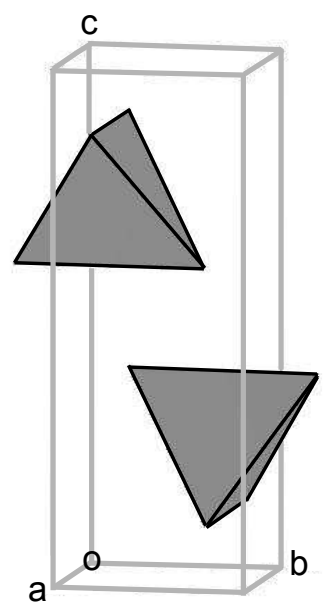

(b)

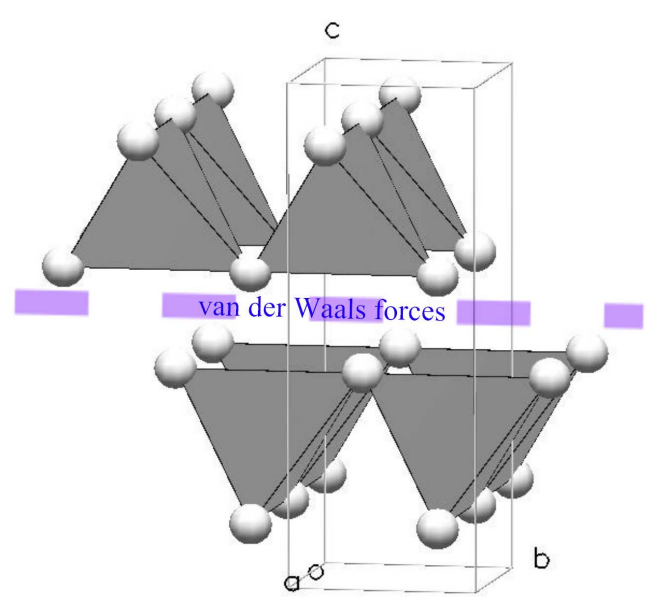

(c)

Figure 3.3. (a) $\mathrm{HgI}_{2}$ unit cell, with the colored mercury ions in orange and iodine ions in purple constituting the ions inside the unit cell. (b) When the tetrahedral coordination about a mercury ion is completed, two tetrahedra, one for each layer, are formed. The structures shown in Figs. (a)-(b), except the highlights, were created using DRAWxtl [58]. (c) The tetrahedral layers are held together by van der Waals forces. 


\subsection{Prediction of Crystal Morphology}

Crystal habit is a qualitative classification of the overall shape of a crystal. Terms such as platelet, tabular, columnar, dendrite, and so forth, are references to the overall shapes of crystals, hence their crystal habits. A collection of faces (or planes) of equivalent symmetry is called a crystal form. For example, in Fig. 3.4, crystals (a) and (b) have the same crystal habit (tabular), but do not share the same crystal forms, whereas in Fig. 3.5 crystals (a) and (b) have the same crystal forms, but do not have the same crystal habit. The habit of the crystal in Fig. 3.5 (a) is tabular, which the same as the habit of crystals in Fig. 3.4, while the habit of the crystal in Fig. 3.5 (b) is blocky.

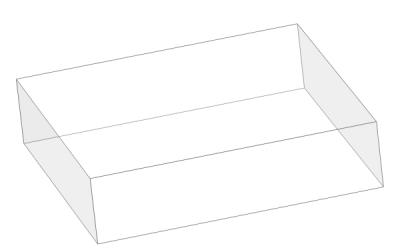

(a)

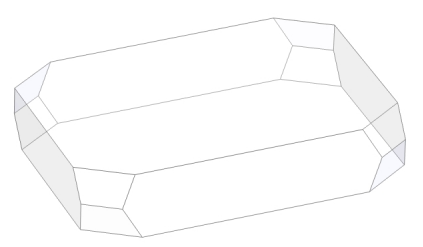

(b)

Figure 3.4. Crystals (a) and (b) have the same habit but not the same forms (or faces).

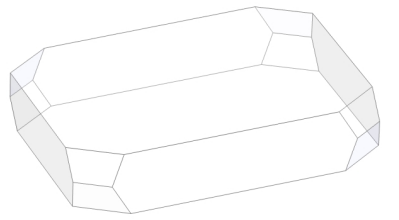

(a)

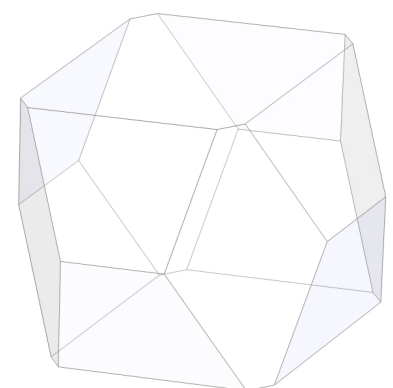

(b)

Figure 3.5. Crystals (a) and (b) have the same forms but not the same crystal habit.

Crystal shape is constructed from or bounded by the slowest growing faces, and generally, morphology is determined by both internal and external influences [59]. Internal influences include crystal structure, dislocations, and internal twin boundaries, while external influences are factors employed in the growth system, such as temperature, pressure, supersaturation, and the presence of impurities. The procedure to determine the influence 
of an external factor on the shape of a grown crystal starts by predicting the theoretical (in many cases, equilibrium) crystal morphology. A summary of methods to determine crystal morphology can be found in many literatures, for example [60]. One method, the Bravais-Friedel-Donnay-Harker (BFDH) method, is reviewed in this study.

\subsubsection{Morphology of Crystal according to the BFDH Rule}

The BFDH method determines the morphology of a crystal based on its structure and symmetry only, not crystal chemistry or surface thermodynamics. Using their method, Bravais and Friedel observed that crystal forms or faces occurring in grown crystals were those with the biggest interplanar distances or $d_{h k l}$ [59]. These crystal faces are said to have the largest morphological importance (MI), determined by the face area and the rate of occurrence in grown crystals. Therefore, the MI of a crystal face $\left(h_{1} k_{1} l_{1}\right)$ when compared to the MI of another crystal face $\left(h_{2} k_{2} l_{2}\right)$ can be written as:

$$
\mathrm{MI}_{h_{1} k_{1} l_{1}}>\mathrm{MI}_{h_{2} k_{2} l_{2}}>\cdots=d_{h_{1} k_{1} l_{1}}>d_{h_{2} k_{2} l_{2}}>\cdots
$$

BFDH-predicted crystal morphology is determined by establishing an inverse proportionality relationship (Eq. (3.2)) between the central distance $r$, which is the distance from the crystal face to the center of the crystal along a path parallel to the normal of the face, and the respective interplanar distance $d_{h k l}$.

$$
r_{h_{1} k_{1} l_{1}}<r_{h_{2} k_{2} l_{2}}<\cdots \propto \frac{1}{d_{h_{1} k_{1} l_{1}}}<\frac{1}{d_{h_{2} k_{2} l_{2}}}<\cdots
$$

In the predicted crystal morphology, the growth rate of a face $(h k l), R_{h k l}$, can be defined as:

$$
R_{h_{1} k_{1} l_{1}}<R_{h_{2} k_{2} l_{2}}<\cdots \propto r_{h_{1} k_{1} l_{1}}<r_{h_{2} k_{2} l_{2}}<\cdots
$$

Therefore, the slower the growth rate of a face, the more significant its morphological importance. 
Furthermore, centering of the unit cell ${ }^{3}$ may halve certain $d_{h k l}$, for which the list is shown in Table 3.1. Moreover, Donnay and Harker [59] observed violations to the BravaisFriedel rule in real crystals, concluding that when screw axes and glide planes existed in a crystal symmetry, they had to be taken into account. According to the Donnay-Harker rules, the interplanar distance, $d_{h k l}$, of a face that is perpendicular to a $2_{1}$ screw axis, or a screw diad axis, is halved. A screw diad axis exists when the crystal configuration is twofold repeated after a translation parallel to the axis and a $180^{\circ}$ rotation. The same rule of $d_{h k l}$ halving exists for $d_{h k l}$ perpendicular to a glide plane, because the crystal configuration is repeated after every $\frac{1}{2} d_{h k l}$. Donnay and Donnay [59] also observed the influence of pseudosymmetry in crystal geometry, which again leads to further sectioning of $d_{h k l}$. Thus, the conditions for halving, trisecting, quartering, and so forth are equal to the conditions that apply to the systematic extinction conditions for x-ray diffraction.

Table 3.1. Symmetry requirement for halving of the interplanar distance $d_{h k l}$ in crystal morphology determination using the BFDH rule.

\begin{tabular}{cc}
\hline Unit cell centering & Condition for $d_{h k l}$-halving \\
\hline$P$ & none \\
$I$ & $h+k+l$ is odd \\
$F$ & $h, k$, and $l$ are not all even or odd \\
$C$ & $h+k$ is odd \\
$B$ & $h+l$ is odd \\
$A$ & $k+l$ is odd \\
\hline
\end{tabular}

\subsection{Morphology of $\mathbf{H g I}_{2}$}

In this section, the BFDH rule is described further, using the derivation of the $\mathrm{HgI}_{2}$ morphology as an example. Because of the primitive centering in the $\mathrm{HgI}_{2}$ unit cell (see Sec. 3.1), according to Table 3.1, that centering does not render any condition for $d_{h k l}$ halving.

\footnotetext{
${ }^{3}$ Centering of a unit cell refers to the position of the lattice points. $P=$ primitive, $I=$ body centered, $F=$ (all-)face centered, $A=A$-face centered, $B=B$-face centered, $C=C$-face centered [57]. $A$-, $B$-, and $C$-faces are faces perpendicular to the $a-, b$-, and $c$-axes, respectively.
} 


\subsubsection{According to BFDH rule}

For the tetragonal crystal structure, the interplanar distance $d_{h k l}$ can be found by using Eq. 3.4,

$$
\frac{1}{d_{h k l}^{2}}=\frac{h^{2}+k^{2}}{a^{2}}+\frac{l^{2}}{c^{2}} .
$$

Table 3.2 lists the first seven crystal forms in order from the largest $d_{h k l}$ (for $h, k, l$ $\leq 3$ ). According to Fig. 3.2, a 42 screw axis, n- and c-glide planes are perpendicular to the $\{001\}$ faces, while a $2{ }_{1}$ screw axis is perpendicular to the 100 faces, and when the screw axes and glide planes are taken into account, $d_{001}$ and $d_{100}$ are halved. The order of the morphological importance (MI) according to the BFDH rule is listed in the second to last column in Table 3.2. Accordingly, the list shows that the expected $\mathrm{HgI}_{2}$ crystal faces ordered from the most significant MI are $\{001\},\{101\},\{102\},\{110\},\{103\},\{100\}$, and $\{201\}$.

Table 3.2. The list of $\mathrm{HgI}_{2}$ growth faces as predicted by the BFDH rule along with their corresponding interplanar spacing $d_{h k l}$.

\begin{tabular}{rcccc}
\hline face & $d_{h k l}(\AA)$ & $\frac{1}{2} d_{h k l}(\AA)$ & Order of MI & $r_{h k l}{ }^{4}$ \\
\hline$\{001\}$ & 12.45 & 6.23 & 1 & 1.04 \\
$\{100\}$ & 4.36 & 2.18 & 6 & 2.96 \\
$\{101\}$ & 4.12 & - & 2 & 1.57 \\
$\{102\}$ & 3.57 & - & 3 & 1.80 \\
$\{110\}$ & 3.08 & - & 4 & 2.09 \\
$\{103\}$ & 3.01 & - & 5 & 2.14 \\
$\{201\}$ & 2.15 & - & 7 & 3.00 \\
\hline
\end{tabular}

Using Eq. 3.2, a BFDH-predicted $\mathrm{HgI}_{2}$ morphology can be obtained by taking the inverse of a respective $d_{h k l}$, which is proportional to the central distance $r_{h k l}$, whose values are in the last column of Table 3.2. Thus, the predicted morphology for $\mathrm{HgI}_{2}$ in Fig. 3.6 shows that the crystal habit is tabular, and the visible crystal forms are $\{001\},\{101\}$, and

\footnotetext{
${ }^{4} \mathrm{r}$ is proportional to the inverse of the respective $d_{h k l}$. To predict a $\mathrm{HgI}_{2}$ crystal morphology according to the BFDH rule, these values were normalized by the largest value, then multiplied by 3 to result in the values listed in the last column of Table 3.2
} 
$\{110\}$. Although according to the BFDH rule the $\{102\}$ faces are morphologically more significant than the $\{110\}$ faces, nonetheless, the $\{102\}$ faces are not visible. Ultimately, the combined effects of the predicted value of $r_{h k l}$ and the angle between crystal faces determine certain crystal forms in the final morphology.

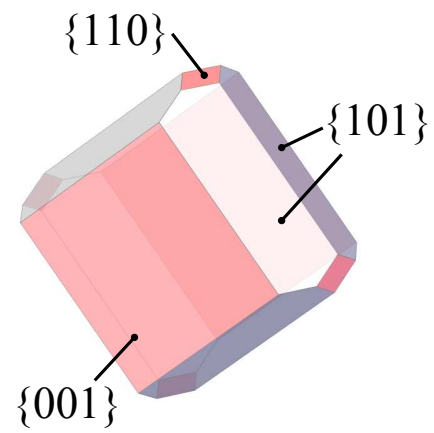

Figure 3.6. The morphology of $\mathrm{HgI}_{2}$ crystal according to the $\mathrm{BFDH}$ rule, showing a tabular crystal habit with the $\{001\},\{101\}$, and $\{110\}$ crystal faces.

The shapes of $\mathrm{HgI}_{2}$ crystals grown in a vertical furnace, as well as $\mathrm{HgI}_{2}$ small single crystals grown in a horizontal furnace without any additive, follow the crystal shape predicted by the BFDH rule, with frequent occurrences of the $\{10 l\}$ faces or crystal forms, and slight variations on the values of the central distances $r_{h k l}$ (hence, variations in the sizes of crystal faces; see Section 5.9.4). Instead of the $\{101\}$ faces predicted by the BFDH rule, it is the $\{102\}$ faces that are usually observed and with large sizes. Although the $\{001\}$, $\{110\}$, and $\{101\}$ crystal forms are observed in $\mathrm{HgI}_{2}$ crystals grown with low molecular weight polyethylene or ketones, the crystals assume the shape of tetragonal prismatic crystals, elongated in the [001] direction and with large $\{110\}$ faces. Explanations on how the low molecular weight polyethylene and ketone modify $\mathrm{HgI}_{2}$ crystal shape are detailed in Section 5.9.5. 


\section{Chapter 4}

\section{Experimental Methods and Procedures}

Fig. 4.1 offers a flow chart of the experimental purification and growth procedures done in-house in the SMART laboratory at Kansas State University. Each step of purification, growth, and material characterization for $\mathrm{HgI}_{2}$ growth by the horizontal furnace will be explained in the following sections.

\section{1 $\quad \mathrm{HgI}_{2}$ Purification Procedures}

$\mathrm{HgI}_{2}$ starting material was purified, primarily, by sublimation in dynamic vacuum, melting, and sublimation in static vacuum (Fig. 4.1). In order to eliminate the probability of undue variations in growth results caused by material quality, all $\mathrm{HgI}_{2}$ starting material had to undergo purification. $\mathrm{HgI}_{2}$ purification methods are an established set of procedures, which were developed in conjuction with $\mathrm{HgI}_{2}$ growth in a vertical furnace [25], and these purification procedures $\mathrm{HgI}_{2}$ are described, in order, in the following sections.

\subsubsection{Purification by Sublimation in Dynamic Vacuum}

As Fig. 4.1 shows, the $\mathrm{HgI}_{2}$ starting material was purified using the established procedures at the former EG\&G Laboratory in Santa Barbara, CA [23]. The starting material was purified through sublimation in dynamic vacuum to gradually remove volatile impu- 


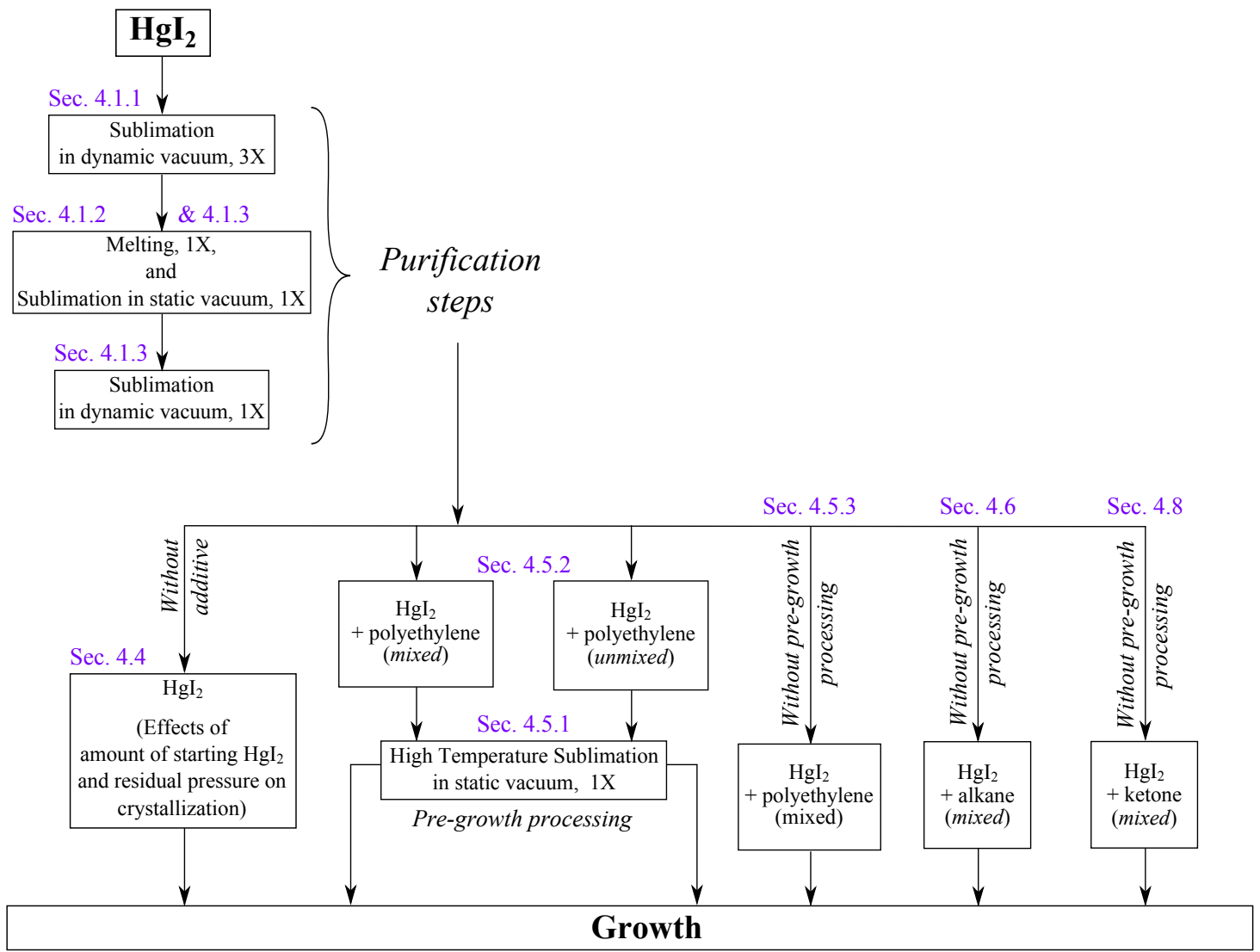

Figure 4.1. Chart of the experimental procedures of the purification steps followed by the growth steps, with the respective sections in the text labeled in purple.

rities with higher vapor pressures than that of $\mathrm{HgI}_{2}$ [23]. Fig. 4.2 details the equipment used in the process and Fig. 4.3 diagrams the process of sublimation by dynamic vacuum schematically. A Pfeiffer turbo molecular vacuum pump, backed by a Pfeiffer rotary vane pump, maintained a dynamic vacuum of $<5 \times 10^{-3}$ Torr in the ampoule. The sublimation chamber consisted of a Pyrex glass ampoule connected through an O-ring joint to a bell jar. A Teflon sleeve was located in the bell jar and extended into the ampoule. During a sublimation run, most of the ampoule's length was in a one-zone furnace (Fig. 4.3), acting as the hot zone. The O-ring part of the ampoule and the bell jar were located outside of the furnace, hence the cold zone. Finally, the bell jar was connected through a ball joint to a Pyrex glass vapor trap, which was immersed in $\mathrm{LN}_{2}$ cooled water during a sublimation run. Depending on the amount of $\mathrm{HgI}_{2}$ being sublimed, a single run can last between 
three and five days. After every sublimation run, $\mathrm{HgI}_{2}$ that condensed in the Teflon sleeve was retrieved for subsequent sublimation or for melting. Otherwise $\mathrm{HgI}_{2}$ was retrieved and recycled for another sublimation run.

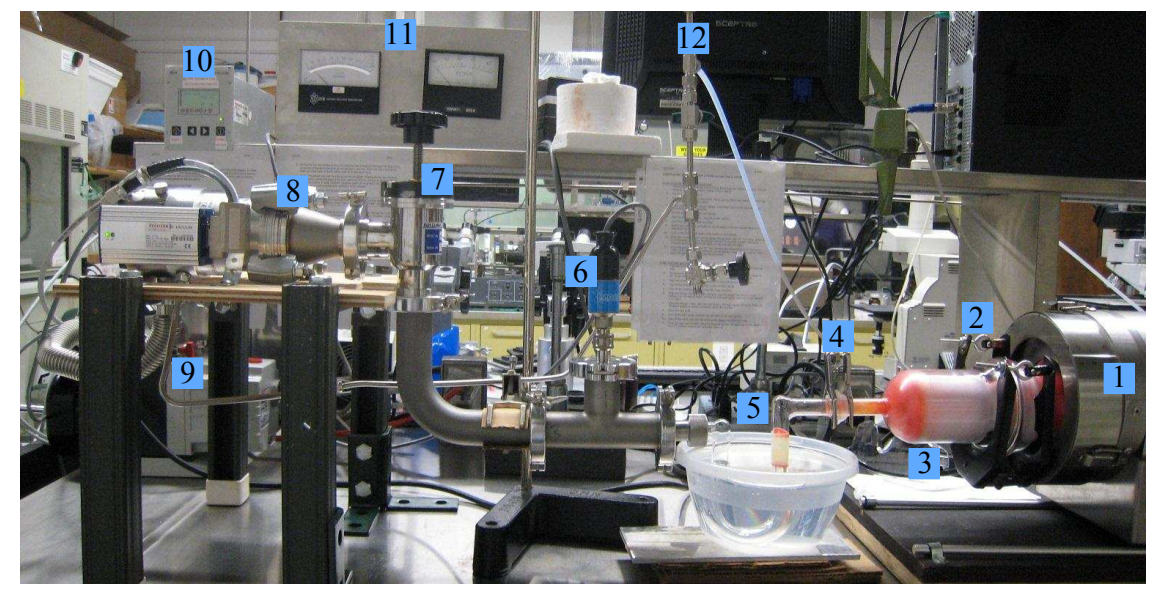

Figure 4.2. The equipment used for purifying $\mathrm{HgI}_{2}$ by dynamic sublimation: (1) one zone furnace, (2) clamp, (3) Teflon sleeve, (4) clamp, (5) cold trap, (6) pressure gauge, (7) valve, (8) turbomolecular pump, (9) mechanical pump, (10) turbomolecular pump controller, (11) pressure gauge meter, (12) $\mathrm{N}_{2}$ line.

After material retrieval, the glass ampoule, the bell jar, the Teflon sleeve, and the glass cold trap were rinsed with acetone and then isopropanol. These steps were followed by immersion in $1 \%$ potassium iodide $(\mathrm{KI})$ solution. The apparatus were then cleaned and rinsed thoroughly with deionized (DI) water. Next, the glass ampoule and the bell jar were etched in $1 \%$ hydrofluoric acid (HF) solution for 20 minutes, followed by a thorough repeated rinsing with DI water. Then, the glass ampoule was annealed and dried at $550^{\circ} \mathrm{C}$ in a dedicated furnace tube while the bell jar was dried in an oven set to at least $105^{\circ} \mathrm{C}$. Finally, the glass cold trap was air dried.

\subsubsection{Purification by Melting}

The product of the third sublimation run was retrieved and ground with a glass mortar and pestle (cleaned by the same procedure as other glass apparatus in contact with $\mathrm{HgI}_{2}$ ). Using a glass funnel, the pulverized $\mathrm{HgI}_{2}$ was poured into the first chamber in a doublechamber melting ampoule (see Fig. 4.4). For sealing, the melting ampoule was mounted at 


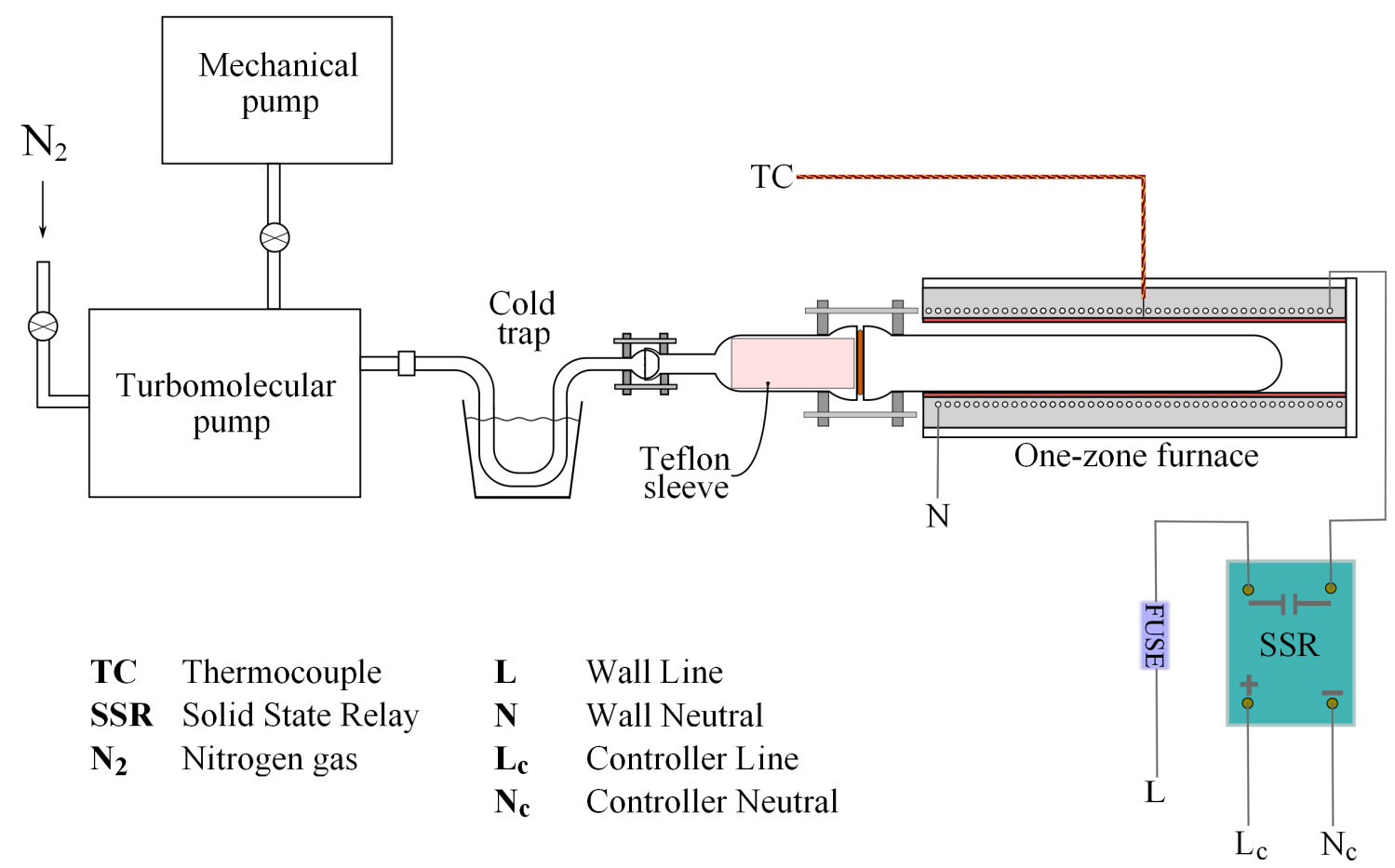

Figure 4.3. Schematics of the equipment used in purification by dynamic sublimation.

the ampoule station, which was equipped with a mechanical rotary pump and a two-stage sorption pumping system. This system is able to evacuate an ampoule to a vacuum $<1 \mathrm{x}$ $10^{-3}$ Torr. After the desired vacuum level was reached, the ampoule was sealed using a natural gas and oxygen hand torch.

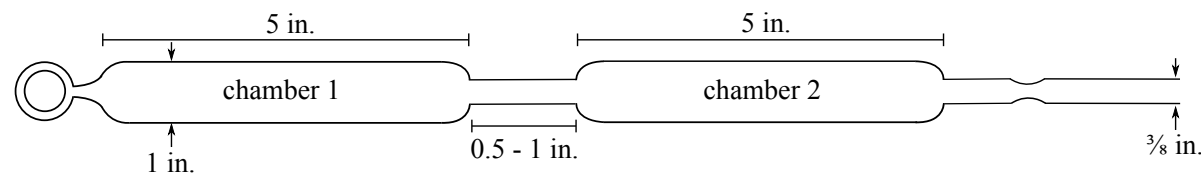

Figure 4.4. Length-wise cross section of a double chamber ampoule, used for $\mathrm{HgI}_{2}$ melting (in chamber 1) and static sublimation (from chambers 1 to 2), following the purification by sublimation in dynamic vacuum. A depression at the tube-mouth indicates the sealing location.

After sealing, the melting ampoule was placed in a two-zone horizontal furnace (also used for growth runs) with the filled, first chamber positioned in Zone 2. This step was completed to sublime residual $\mathrm{HgI}_{2}$ material from the second chamber into the first chamber, where $\mathrm{HgI}_{2}$ melting was to be peformed (Fig. 4.5(a)). Before ampoule insertion, the 
furnace zones had been set at temperatures of $120^{\circ} \mathrm{C}$ and $100^{\circ} \mathrm{C}$ for Zones 1 and 2 , respectively. These temperatures were kept for 12 hours prior to starting the melting procedure to drive all material from the second chamber to the first chamber for melting. Both zones were increased by $1^{\circ} \mathrm{C} /$ minute to $350^{\circ} \mathrm{C}$. After the zones reached $350^{\circ} \mathrm{C}$, the melting ampoule was left at this temperature for 24 hours and then rotated $180^{\circ}$ and left in the furnace for 24 more hours. At the end of the melting procedure, the temperatures were set back to $120^{\circ} \mathrm{C}$ and $100^{\circ} \mathrm{C}$ for Zones 1 and 2 , respectively. This step solidified the melted charge in the first chamber (Fig. 4.5(b)).

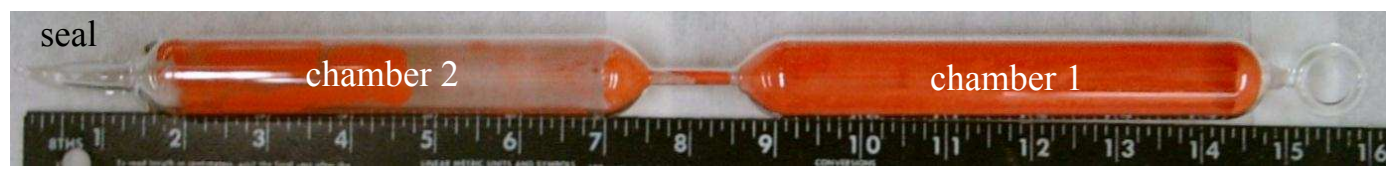

(a)

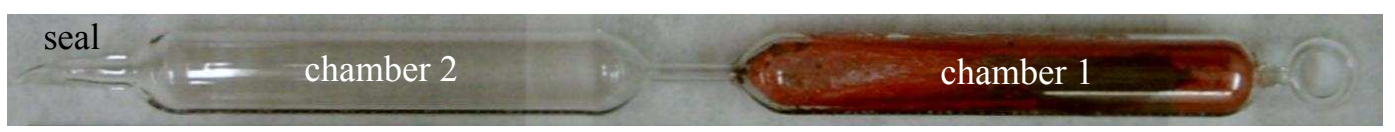

(b)

Figure 4.5. (a) A double chamber ampoule after chamber 2 was filled with pulverized $\mathrm{HgI}_{2}$ and the ampoule subsequently sealed. The sealed ampoule was placed in a two-zone furnace, with chamber 2 placed in the hot zone $\left(\right.$ at $120^{\circ} \mathrm{C}$ ) and chamber 1 in the cold zone (at $100^{\circ} \mathrm{C}$ ), to drive residual pulverized $\mathrm{HgI}_{2}$ from chamber 2 to chamber 1. (b) A double chamber ampoule after completing the melting procedure.

\subsubsection{Purification by Sublimation in Static Vacuum}

The melting ampoule was placed in a one-zone furnace (Fig. 4.6, also shown schematically in Fig. 4.7), to sublime the charge from the first chamber to the second. After all of the charge was moved to the second chamber (Fig. 4.8), the ampoule was removed, and then the ampoule bridge was cut open to separate the first chamber from the second. The material in the second chamber was removed by placing three - four such chambers in the sublimation ampoule (Fig. 4.9) and conducting sublimation in dynamic vacuum, with the same previously described procedures. 


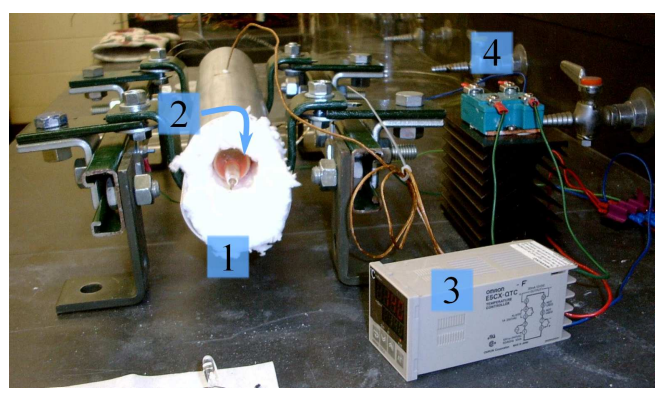

Figure 4.6. (1) Static sublimation ampoule. (2) A double chamber ampoule, with chamber 2 visible. (3) Temperature controller. (4) Solid state relay.

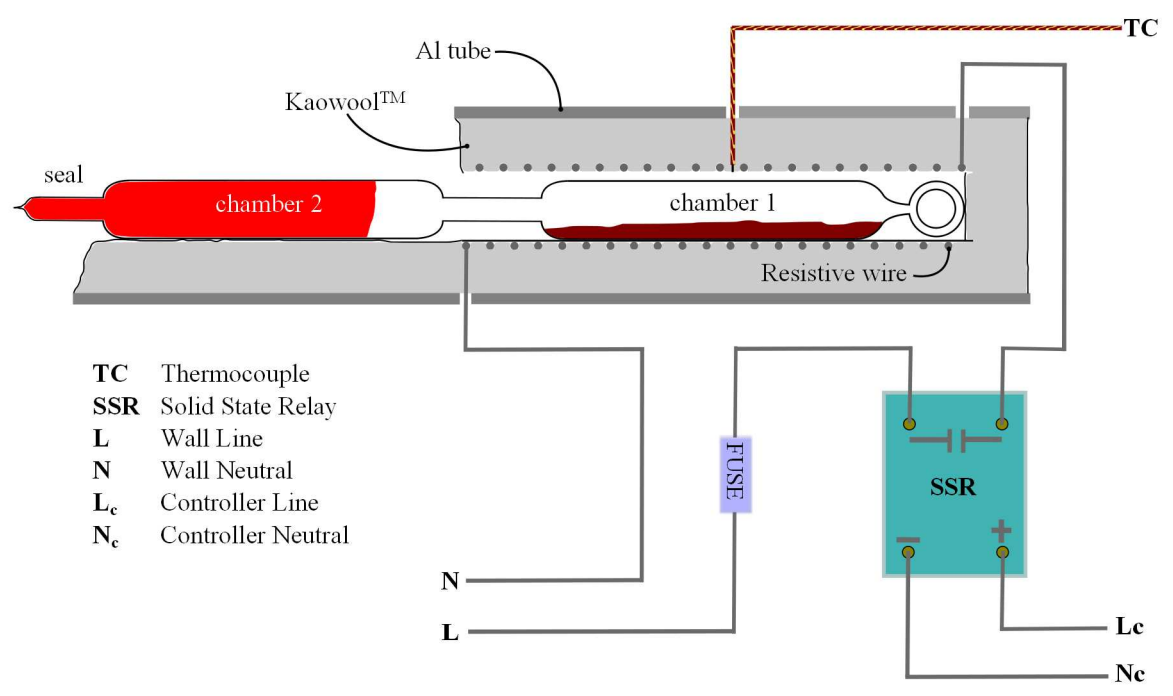

Figure 4.7. The double chamber ampoule in the static sublimation furnace.

\subsubsection{Impurity characterization of $\mathbf{H g I}_{2}$ starting material}

Samples of materials from each purification stage were collected and sent to EAG Laboratories (formerly Shiva Technologies, Inc.) for impurity analysis with glow discharge mass spectroscopy (GDMS). The analyzed sample labels, placed in groups with respect to the starting $\mathrm{HgI}_{2}$ since it was purified in batches, and their information are listed in Table 4.1. 


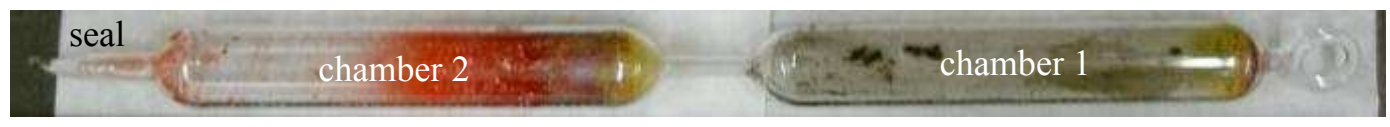

Figure 4.8. The double chamber ampoule (also seen in Fig. 4.5), after $\mathrm{HgI}_{2}$ sublimation in static vacuum, from chamber 1 to chamber2. Left behind in chamber 1 were black carbonaceous impurities.

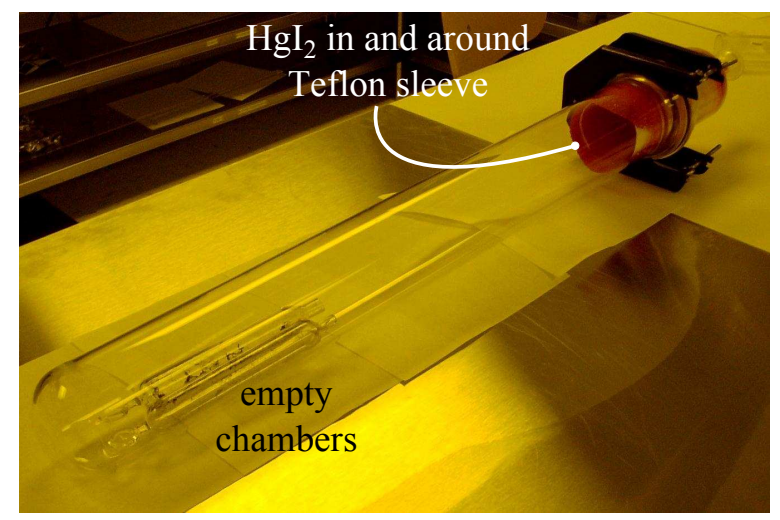

(a)

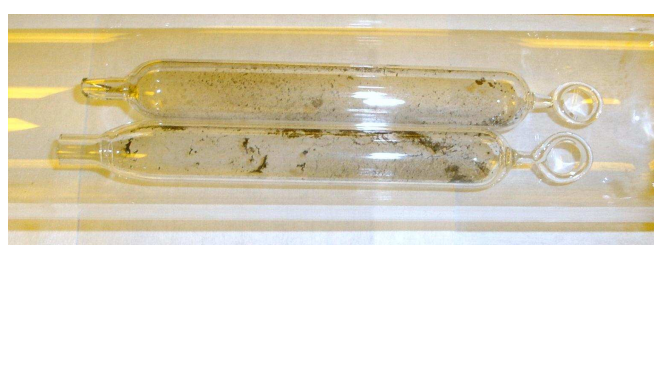

(b)

Figure 4.9. $\mathrm{HgI}_{2}$ retrieval from melting ampoules by sublimation in dynamic vacuum (note that the melting ampoules in (a) and (b) were first generation, one-chamber ampoules, however, the method for $\mathrm{HgI}_{2}$ retrieval from the melting chamber remains the same.): (a) The ampoule used in sublimation in dynamic vacuum, with sublimed $\mathrm{HgI}_{2}$ in or around the teflon sleeve, and empty chambers in the source end. (b) When the $\mathrm{HgI}_{2}$ sublimed from the chambers, carbonaceous impurities were left in the one-chamber ampoules, however, because the impurities did not strongly adhere to the ampoule wall, they were easily included in the sublimed $\mathrm{HgI}_{2}$. The inclusion of impurities in the sublimed $\mathrm{HgI}_{2}$ was the reason behind the utilization of the double-chamber ampoule and the purification procedures described in Figs.4.4 - 4.8 .

\subsection{Ampoule Preparation and Sealing Procedures}

After a considerable amount of time was spent purifying the starting $\mathrm{HgI}_{2}$ material, it was of utmost importance to keep the growth apparatus as free from impurities as possible, moreover to avoid variations in growth results caused by material quality. The practice thus includes glass handling with gloved hands, as well as use of lint-free wipes, DI-water, and high purity solvents. In this section, growth ampoule preparation, which includes cleaning as well as annealing, and its sealing procedures are described. 
Table 4.1. $\mathrm{HgI}_{2}$ samples analyzed by GDMS.

\begin{tabular}{|c|c|}
\hline & Samples \\
\hline Group 1 & $\begin{array}{c}\mathrm{HgI}_{2} \text { starting material } \\
\mathrm{HgI}_{2} \text { after dynamic sublimation no. } 1 \\
\mathrm{HgI}_{2} \text { after melting }\end{array}$ \\
\hline Group 2 & $\begin{array}{l}\mathrm{HgI}_{2} \text { after dynamic sublimation no. } 2 \\
\mathrm{HgI}_{2} \text { after dynamic sublimation no. } 3\end{array}$ \\
\hline Group 3 & $\begin{array}{l}\mathrm{HgI}_{2} \text { before } \mathrm{PE} \text { addition } \\
\mathrm{HgI}_{2} \text { after } \mathrm{PE} \text { addition }\end{array}$ \\
\hline
\end{tabular}

\subsubsection{Ampoule Preparation}

$\mathrm{HgI}_{2}$ growth was conducted in a long Pyrex ampoule, which was sealed using a glass plug (both are schematically illustrated in Fig. 4.10). Before use, both the ampoule and the plug were rinsed with DI water and etched in $1 \%$ HF solution for 15 to 20 minutes. After thorough rinsing with DI water, the glasswares were annealed at $550^{\circ} \mathrm{C}$ for at least six hours in a horizontal furnace. Following subsequent cooling and retrieval from the furnace, to prevent particulate contamination, the mouths of the ampoules were wrapped with parafilm, while the glass plugs were wrapped in lint-free cleanroom wipes.

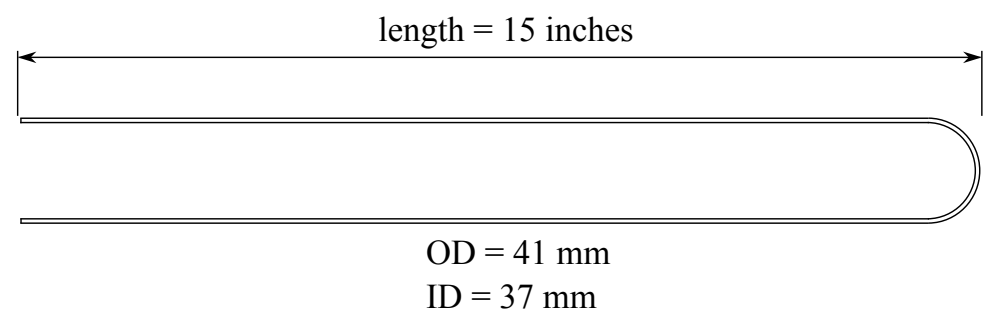

(a)

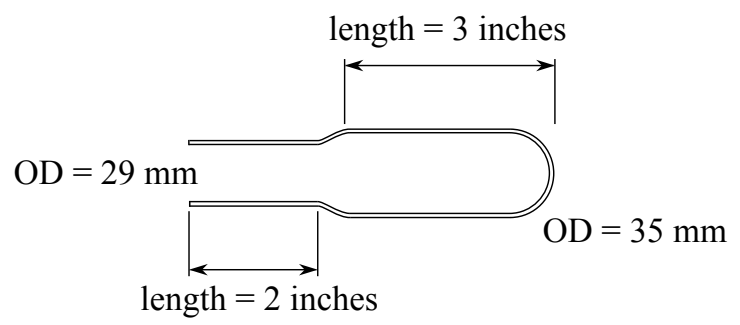

(b)

Figure 4.10. (a) Length-wise cross section of a growth ampoule for $\mathrm{HgI}_{2}$ growth in a horizontal furnace. (b) Length-wise cross section of a plug for use in sealing the growth ampoule. 


\subsubsection{Ampoule Station}

The ampoule station was constructed to combine a gas manifold and several sealing ports to enable high vacuum evacuation capability, as shown in Fig. 4.11. Research grade gas sources were obtained, and then gas-specific purifiers were installed. Quick connect tube fittings secured glass ampoules at the sealing ports accommodating the following outer glass diameters: 3/8", 5/8", and $41 \mathrm{~mm}$.

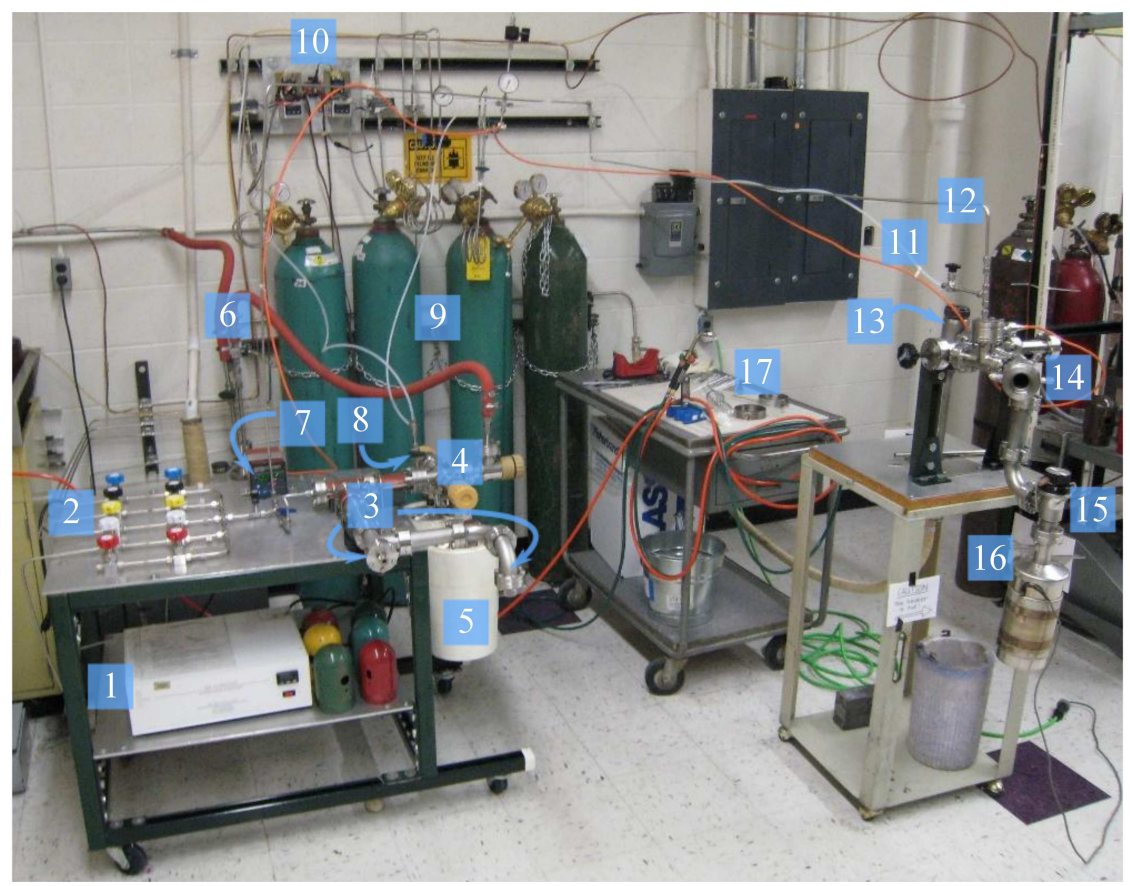

Figure 4.11. (1) $\mathrm{H}_{2}$ gas purifier. (2) Gas manifold. (3) Sealing ports that accomodate at least two ampoule diameters. (4) Valves connected to two sorption pump and a mechanical pump. (5) Sorption pumps in $\mathrm{LN}_{2}$ dewars. (6) Valves and connections to the mechanical pump. (7) Pressure gauge meter. (8) $\mathrm{N}_{2}$ line. (9) Gas cylinders. (10) Temperature controllers for gas purifiers. (11) $\mathrm{N}_{2}$ line. (12) Connection to the gas manifold. (13) Valve to the mechanical pump. (14) Sealing port that accomodates a $41 \mathrm{~mm}$ ampoule. (15) Valve to a sorption pump. (16) Sorption pump with a wrap-around heater. (17) Pyrex glasswork bench.

For chamber evacuation at low vacuum, the ampoule station was connected to an oilsealed mechanical pump. Notably, vacuum level lower than $10^{-3}$ Torr can be achieved with one stage or two-stage sorption pumps. Meanwhile, at the first ampoule station, where the gas manifold is, the vacuum level was measured with a Piezoresistive-Pirani gauge (Teledyne Hastings model no. HPM2002), and at the second ampoule station the vacuum level 
was measured with a Pirani-cold cathode gauge (Pfeiffer model no. PKR251-PTR26002).

\subsubsection{Sealing Procedures}

Ampoules are sealed using a torch with a natural gas and oxygen flame. For sealing a growth ampoule illustrated in Fig. 4.10, pre- and post-sealing flame annealing at and around the sealing area were performed to reduce thermal stress that could lead to cracking. The filled ampoule was evacuated to $\sim 0.2$ Torr and followed by a $\mathrm{N}_{2}$ backfill. This rough vacuum-and- $\mathrm{N}_{2}$ backfill stage was repeated three times and then followed by a final rough vacuum stage and additional evacuation with a sorption pump to a desired vacuum level of $\sim 10^{-4}$ Torr. After being sealed, the ampoule was allowed to cool at the ampoule station for two hours until the glass and fittings could be handled.

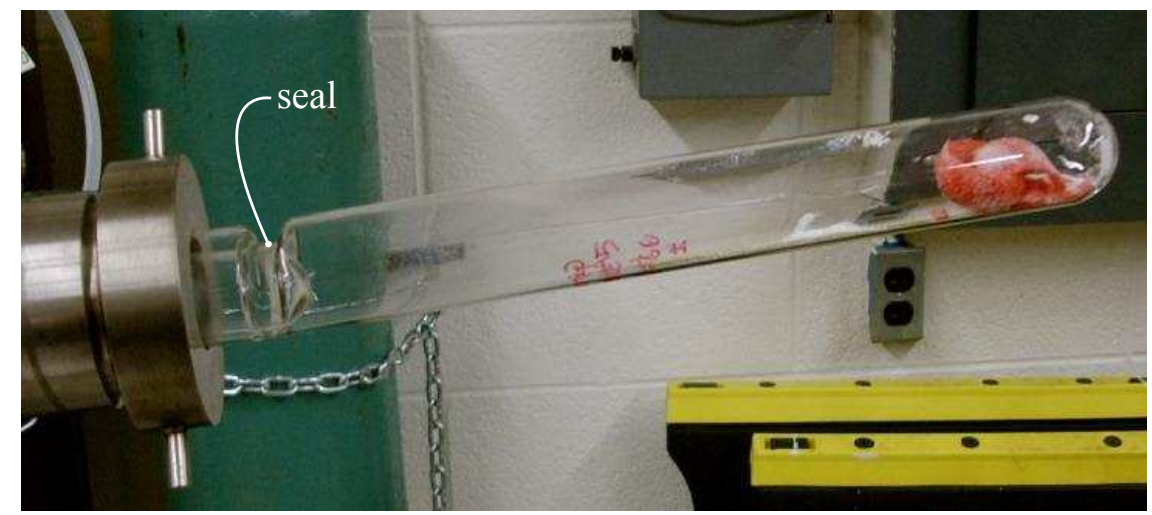

Figure 4.12. A sealed ampoule at the sealing port after the sealing procedure. This particular ampoule was an ampoule for the pre-growth polyethylene processing. The same ampoule-plug combination and sealing procedure were used for growth ampoules.

\subsection{Horizontal Furnaces}

The laboratory has thirty-two two-zone furnaces for growth and additional processes concerning $\mathrm{HgI}_{2}$. These furnaces, along with an additional eight one-zone furnaces with the same total zone length and dimensions as the two-zone furnaces, were donated by TN Technologies (Round Rock, Texas). These forty horizontal furnaces were arranged in 
shelves constructed on-site in the SMART Laboratory, using Unistrut parts and pieces, and aluminum sheets. The one-zone furnaces were not employed for any of the growth runs or processes in this study, nevertheless, they were included in the installation process. The configuration and wiring schematics of every two-zone furnace is shown in Fig. 4.13. The wiring schematics of the one-zone furnaces is not shown but it is equivalent to one set of thermocouple (TC), solid state relay (SSR), and controller employed in the two-zone furnaces.

Analog PID controllers for use with the furnaces were also donated by TN Technologies, however, installation of digital PID controllers were decided upon, for better temperature control and usability. Each zone in ten of the 2-zone furnaces was retrofitted by individual Eurotherm PID $^{1}$ controllers, while each zone in the other thirty furnaces was controlled by ADAPT micro-controller developed by the Mellen Company. Both types of the controllers were purchased from the respective vendors, and they were installed on-site by SMART Laboratory personnel.

The PID settings in the individual Eurotherm conrollers were optimized using the autotune feature in the controllers, while the PID settings in the micro-controllers were received already tuned by the manufacturer, given the specifics of the furnaces to be controlled. One end of the heating coil of each zone in the 2-zone furnaces controlled by Eurotherm PID controllers was connected to the $120 \mathrm{~V}$ wall line $(\mathbf{L})$, through a solid state relay (SSR), while the other end was connected to the neutral line $(\mathbf{N})$. Because the PID settings in the ADAPT micro-controllers were optimized for furnaces operated at $800^{\circ} \mathrm{C}$ or above, the input power to each zone connected to the ADAPT micro-controllers had to be steppeddown using a transformer, from $120 \mathrm{VAC}$ to $24 \mathrm{VAC}$. The power reduction was necessary to tightly control the process temperature within $0.1^{\circ} \mathrm{C}$ of the temperature setpoint.

\footnotetext{
${ }^{1}$ PID stands for proportional-integral-derivative, which refers to the variables used in the controller's algorithm to minimize error between the temperature setpoint and the processed value (i.e., the observed temperature).
} 


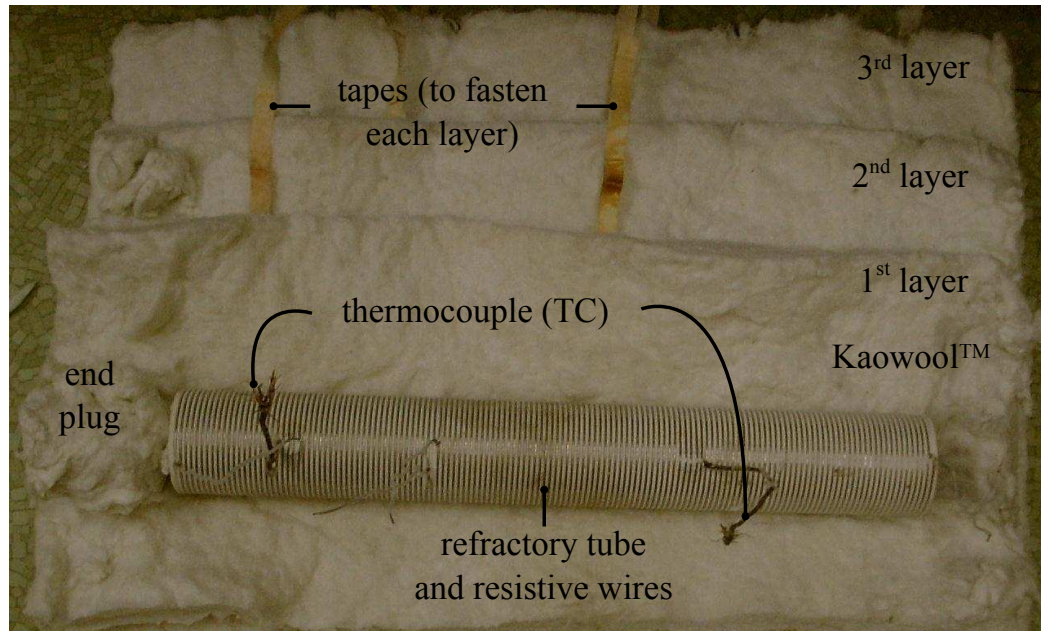

(a)

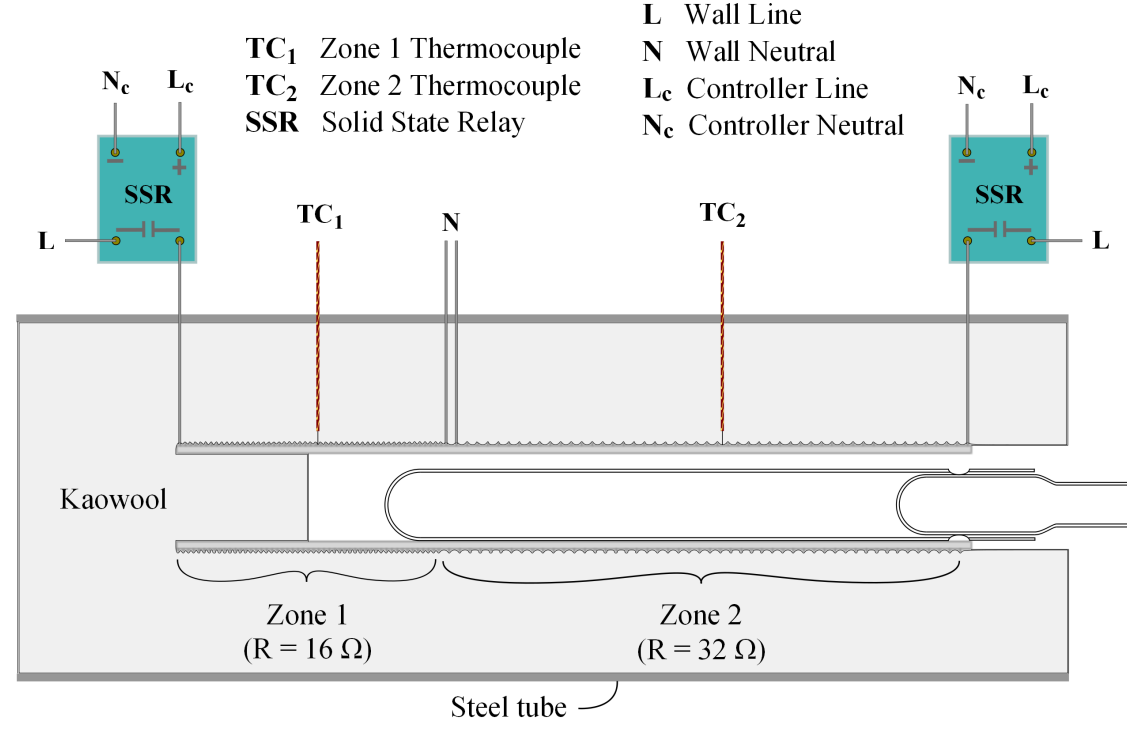

(b)

Figure 4.13. (a) Parts that constitute a two-zone horizontal furnace tube used in the growth experiments. (b) Schematics of a sealed growth ampoule in a two-zone growth furnace. Each zone has its own controller, either by a microcontroller or by a combination of SSRPID controller units. 


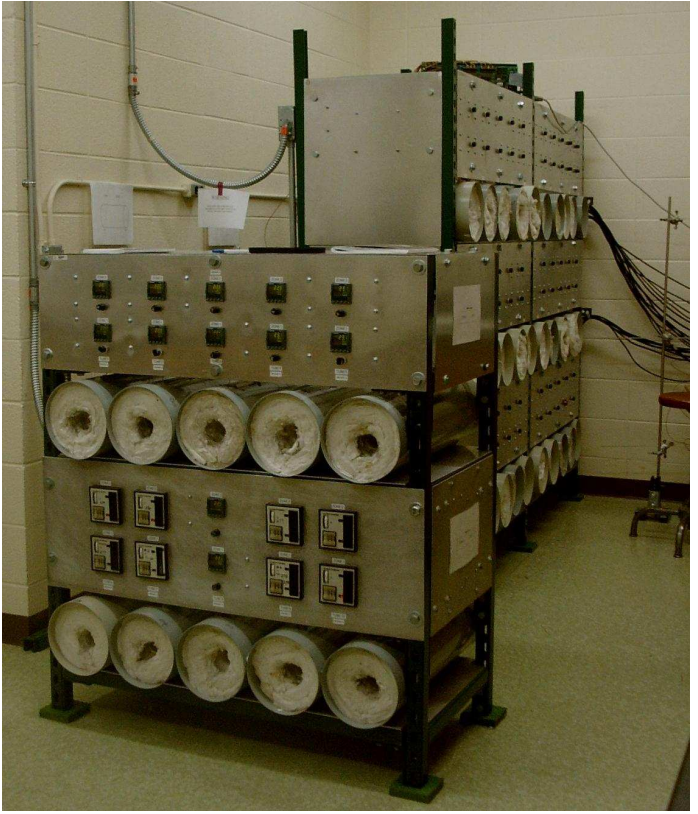

(a)

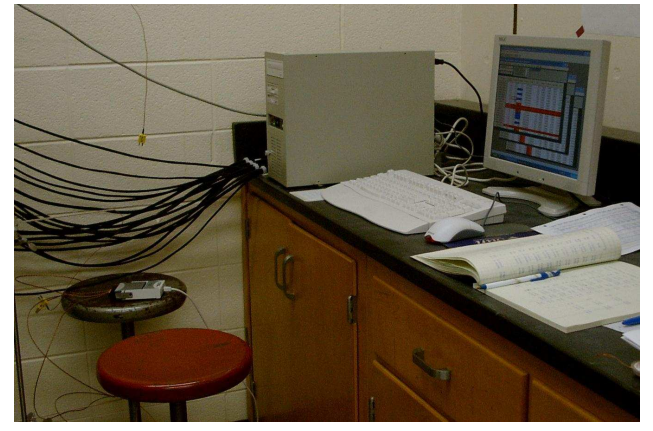

(b)

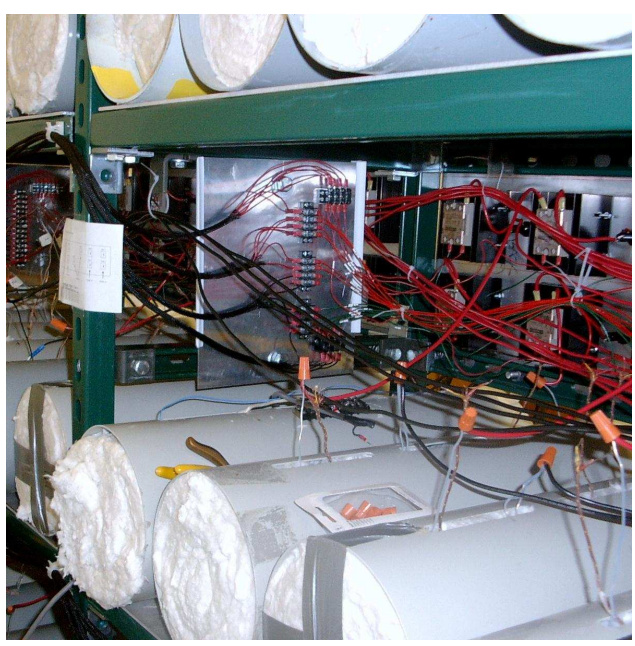

(c)

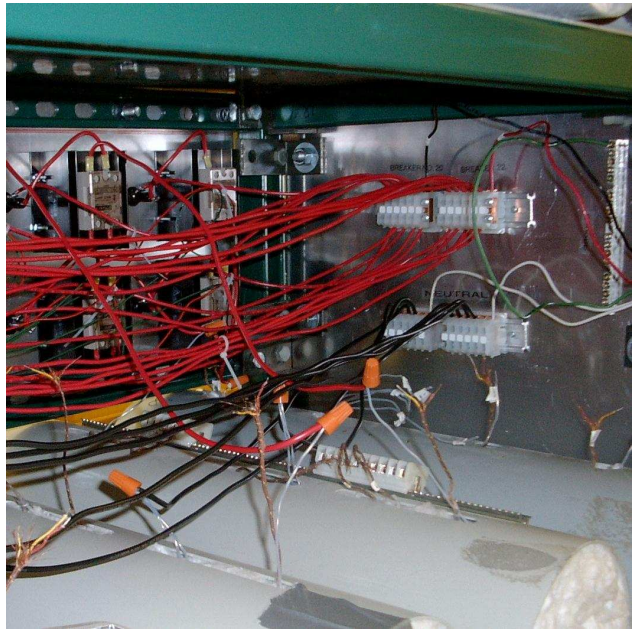

(d)

Figure 4.14. (a) Horizontal furnaces used for pre-growth processing and growth experiments. (b) The computer used to micro-control temperatures in 30 furnaces. (c) \& (d) The wiring setup for the horizontal furnaces. 


\subsection{Growth With No Additive}

Two sets of growth runs without additive, but with purified $\mathrm{HgI}_{2}$ were performed to facilitate a comparison between $\mathrm{HgI}_{2}$ crystals grown with and without additive. In the first set of growth, the amount of the starting $\mathrm{HgI}_{2}$ was varied, and in the second set of growth, a residual pressure (allowing a total pressure inside the ampoule to be between 1 and 10 Torr). These experiments were performed without organic additive to observe the size and the deposition pattern of crystals in the crystallization region during $\mathrm{HgI}_{2}$ growth in a twozone horizontal furnace. The growth with residual pressure was performed specifically to prove claims put forward in [32] that a total pressure between 1 and 10 Torr is required to obtain $\mathrm{HgI}_{2}$ platelets.

For the growth runs in the first set (in high vacuum, i.e., low pressure), 80 grams and 40 grams of the starting $\mathrm{HgI}_{2}$ were used. Each amount of $\mathrm{HgI}_{2}$ was placed into an ampoule and sealed under vacuum and then grown with Zone 1 at $100^{\circ} \mathrm{C}$ and Zone 2 at $80^{\circ} \mathrm{C}$. Meanwhile, the growth run in the residual ampoule used 80 grams of the starting $\mathrm{HgI}_{2}$. After high vacuum was reached, the ampoule was backfilled with $\mathrm{N}_{2}$ gas up to 4.2 Torr (gauge). The ampoule was then sealed as previously described.

\subsection{Growth With Polyethylene}

Previous studies on $\mathrm{HgI}_{2}$ growth with polyethylene [28-30] did not detail what type of polyethylene was employed, hence, definitive conclusions on how polyethylene affects $\mathrm{HgI}_{2}$ crystal morphology could not be drawn. The proceeding sections detail $\mathrm{HgI}_{2}$ growth runs utilizing different types of polyethylene, namely, the low molecular weight polyethylene, the ultra high molecular weight polyethylene, and spectrophotometric grade polyethylene. 


\subsubsection{Pre-growth polyethylene processing step}

Three types of polyethylene were used in the following experiments: spectrophotometric grade polyethylene, low molecular weight polyethylene $\left(\overline{\mathrm{M}}_{\mathrm{w}} \sim 4 \times 10^{3} \mathrm{~g} / \mathrm{mol}\right)$, and ultra high molecular weight polyethylene $\left(\overline{\mathrm{M}}_{\mathrm{w}} \sim 3 \times 10^{6}-6 \times 10^{6} \mathrm{~g} / \mathrm{mol}\right)$. The information on these polyethylene types is listed in Table 4.2. For the polyethylene processing stage (Section 4.5.2), the starting $\mathrm{HgI}_{2}$ and each polyethylene type were either mixed directly or loaded into separate vials.

Following the recommendation of TN Technologies, a pre-growth polyethylene processing was carried out (Fig. 4.1). Although a reason for the inclusion of this step was unclear, thermal desorption (TD)-GC/MS analysis of the low $\overline{\mathrm{M}}_{\mathrm{w}}$ polyethylene (with results described in Section 5.7.1) reveals that there are chemicals desorbed from the polyethylene when heated at temperatures employed during the pre-growth polyethylene processing stage, thus shedding a light on why this step is recommended.

Table 4.2. Types of polyethylene used in $\mathrm{HgI}_{2}$ growth experiments.

\begin{tabular}{ccc}
\hline Type of polyethylene & $\overline{\mathrm{M}}_{\mathrm{w}}\left(\mathrm{g} \mathrm{mol}^{-1}\right)$ & Source \\
\hline Low $\overline{\mathrm{M}}_{\mathrm{w}}$ & $4 \times 10^{3}$ & Sigma Aldrich \\
Ultrahigh $\overline{\mathrm{M}}_{\mathrm{w}}$ & 3 to $6 \times 10^{6}$ & Sigma Aldrich \\
Spectrophotometric grade & N/A & Sigma Aldrich \\
\hline
\end{tabular}

For the pre-growth processing step with mixed starting materials, $\mathrm{HgI}_{2}$ and polyethylene starting materials were simultaneously loaded into an ampoule, which was then sealed under vacuum. The ampoule was placed in a two-zone horizontal furnace, with the hot zone (Zone 1) set at $230^{\circ} \mathrm{C}$ and the deposition zone set at $150^{\circ} \mathrm{C}$. In this step, all the starting $\mathrm{HgI}_{2}$ usually sublimed to the deposition zone within 5 to 10 days, depending on the amount of polyethylene and sealing quality.

For the pre-growth processing step with un-mixed starting materials, $\mathrm{HgI}_{2}$ was placed in a glass vial and polyethylene powder was placed in another vial. Next, both vials were placed in an ampoule subsequently sealed under vacuum. The ampoule was placed in a two-zone horizontal furnace using the same setting as in the process with a mixed source. The rest of the procedure was the same as the one previously mentioned. 
For both cases of source condition, $\mathrm{HgI}_{2}$ was retrieved and placed in a new (growth) ampoule, which was subsequently sealed under vacuum. Prior to the growth stage, the ampoule was placed in reverse (the source end was placed in Zone 2 while the cold end was in Zone 1). This stage was to ensure that all $\mathrm{HgI}_{2}$ sublimed from the entire length of the furnace and condensed in the source end of the ampoule. The growth period was five days. After growth, the ampoule was retrieved and allowed to cool to room temperature through natural convection.

\subsubsection{Growth with the pre-growth polyethylene processing step}

$2^{3}$ factorial analysis was performed on a set of growth runs with spectrophotometric grade polyethylene. The variables were the source temperature $\mathbf{T}$ (Zone 1 temperature), the temperature gradient $\Delta \mathbf{T}$ (the difference between the temperatures of Zones 1 and 2), and the amount of additive $\mathbf{M}$ (in wt- $\%$ with respect to the amount of $\mathrm{HgI}_{2}$ ). The factors kept constant were the amount of starting $\mathrm{HgI}_{2}$ and the type of polyethylene. The length of the deposition zone was the response or measured factor that would be analyzed. The variables are listed in Table 4.3

Table 4.3. List of variables in factorial analysis of growth with spectrophotometric grade polyethylene.

\begin{tabular}{ccc}
\hline Factors & - & + \\
\hline Source temperature $\mathbf{T}\left({ }^{\circ} \mathbf{C}\right)$ & 90 & 100 \\
Temperature gradient $\Delta \mathbf{T}\left({ }^{\circ} \mathbf{C}\right)$ & 10 & 20 \\
Amount of polyethylene $\mathbf{M}(\mathrm{wt}-\%)$ & 1 & 2 \\
\hline
\end{tabular}

\subsubsection{Growth without the polyethylene processing step}

A few ampoules were prepared for growth with polyethylene that bypassed the polyethylene processing step (Section 4.5.1). In this set of growth runs, 40.0 grams of the starting $\mathrm{HgI}_{2}$ was directly mixed with the low $\overline{\mathrm{M}}_{\mathrm{w}}$ polyethylene. Three values of polyethylene amounts were prepared, each for each growth run: $0.02,0.1$, and $5 \mathrm{wt}-\%$. The 
same ampoule sealing procedure was employed, but the ampoule reversal step prior to growth was not done. Then, each ampoule was placed in a two-zone furnace with Zone 1 at $100^{\circ} \mathrm{C}$ and Zone 2 at $80^{\circ} \mathrm{C}$ for five days. After growth, the ampoule was retrieved and let to cool to room temperature through natural convection.

\subsection{Growth with n-Alkanes}

Three types of alkanes were used for various growth runs: n-hexatriacontane $\left(\mathrm{C}_{36} \mathrm{H}_{74}\right)$, n-tetracontane $\left(\mathrm{C}_{24} \mathrm{H}_{50}\right)$, and n-eicosane $\left(\mathrm{C}_{20} \mathrm{H}_{42}\right)$. The information on the alkanes is listed in Table 4.4. The starting $\mathrm{HgI}_{2}$ (each about $40.0 \mathrm{~g}$ ) and each n-alkane type (each about 1 wt-\%) were mixed directly in a growth ampoule. The growth with n-alkanes employed no pre-growth processing step (Section 4.5.1) nor the pre-growth ampoule reversal step, but the same ampoule sealing procedure was employed.

Table 4.4. Types of n-alkanes used in $\mathrm{HgI}_{2}$ growth experiments.

\begin{tabular}{cccc}
\hline Type of alkane & & Purity & Source \\
\hline n-hexatriacontane & $\mathrm{n}-\mathrm{C}_{36} \mathrm{H}_{74}$ & $98 \%$ & Sigma Aldrich \\
n-tetracosane & $\mathrm{n}-\mathrm{C}_{24} \mathrm{H}_{50}$ & $99 \%$ & Sigma Aldrich \\
n-eicosane & $\mathrm{n}-\mathrm{C}_{20} \mathrm{H}_{42}$ & $99 \%$ & Sigma Aldrich \\
\hline
\end{tabular}

\subsection{Growth with Ketones}

$\mathrm{HgI}_{2}$ growth runs with ketones commenced after the TD-GC/MS results were obtained (Section 5.7.1). Two types of ketones were utilized: 3-hexadecanone and 14-heptacosanone. The information on the ketones is listed in Table 4.5. Similar to the growth with nalkanes, the starting $\mathrm{HgI}_{2}$ and each type of ketone were mixed directly in a growth ampoule, and the pre-growth processing step (Section 4.5.1) was not used. About $40.0 \mathrm{~g}$ of starting $\mathrm{HgI}_{2}$ was used in each of the growth runs. The details of the growth runs can be found in Table 4.6. 
Table 4.5. Types of ketones used in $\mathrm{HgI}_{2}$ growth experiments.

\begin{tabular}{ccc}
\hline Type of alkane & Purity & Source \\
\hline 3-hexadecanone & $95 \%$ & Sigma Aldrich \\
14-heptacosanone & $97 \%$ & Sigma Aldrich \\
\hline
\end{tabular}

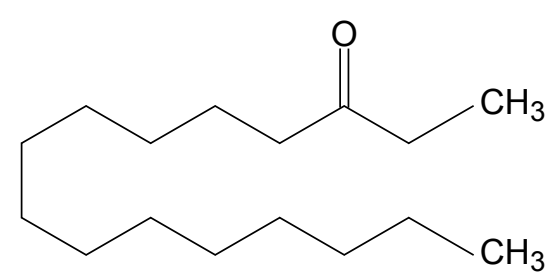

(a) 3-hexadecanone

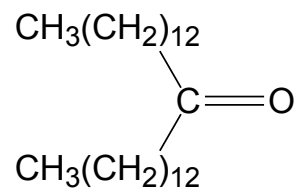

(b) 14-heptacosanone

Figure 4.15. Types of ketones used in $\mathrm{HgI}_{2}$ growth.

\subsection{Material Characterization}

Characterizations were performed on both the starting organic materials and the $\mathrm{HgI}_{2}$ crystals. Thermal desorption followed by gas chromatography coupled with mass spectroscopy (TD-GC/MS) was used to determine the volatiles produced from n-eicosane (n$\mathrm{C}_{20} \mathrm{H}_{42}$ ), low $\overline{\mathrm{M}}_{\mathrm{w}}$ polyethylene, and ultrahigh $\overline{\mathrm{M}}_{\mathrm{w}}$ polyethylene in inert He atmosphere at elevated temperatures up to $150^{\circ} \mathrm{C}$. Attenuated total reflection infrared (ATR-IR) spectrometer was used to examine surfaces of as-grown and freshly cleaved $\mathrm{HgI}_{2}$ crystals grown with low $\overline{\mathrm{M}}_{\mathrm{w}}$ polyethylene.

\subsubsection{TD-GC/MS study polyetylene and n-alkanes}

The TD-GC/MS analysis (the samples were sent for tests conducted by CIBA Corporation) was performed on low $\overline{\mathrm{M}}_{\mathrm{w}}\left(\sim 4 \times 10^{3}\right)$ polyethylene, ultra high $\overline{\mathrm{M}}_{\mathrm{w}}\left(\sim 3 \times 10^{6}-\right.$ $\left.6 \times 10^{6}\right)$ polyethylene, and n-eicosane $\left(\mathrm{C}_{20} \mathrm{H}_{42}\right)$. The polyethylene samples were tested at temperature points of $100^{\circ} \mathrm{C}, 150^{\circ} \mathrm{C}, 200^{\circ} \mathrm{C}$ and $230^{\circ} \mathrm{C}$, while the alkane sample was tested at $100^{\circ} \mathrm{C}$. The low $\overline{\mathrm{M}}_{\mathrm{w}}$ polyethylene was not tested at $200^{\circ} \mathrm{C}$ and $230^{\circ} \mathrm{C}$, because a large amount of volatiles were already detected at lower temperatures. These temperature points were selected in conjunction to the temperatures used during the growth processes (see the 
Table 4.6. $\mathrm{HgI}_{2}$ growth with ketones.

\begin{tabular}{ccc}
\hline Type & Ketone (wt-\%) & $\mathrm{HgI}_{2}(\mathrm{~g})$ \\
\hline 3-hexadecanone & 0.1 & 40.0 \\
14-heptacosanone & 0.1 & 40.1 \\
14-heptacosanone & 1 & 40.2 \\
\hline
\end{tabular}

corresponding temperatures in Section 4.5.2 for the polyethylene pre-processing temperatures and Section 4.6 for growth experiments with alkanes). According to CIBA, for each type of organics, a sample was placed in a thermal desorption tube, where it was subjected to the selected temperatures in succession (the desorption time at each temperature point was 30 minutes). Volatiles produced after a desorption at one temperature point were collected in a cold trap, which was subsequently heated at $20^{\circ} \mathrm{C}$ /minute to $280^{\circ} \mathrm{C}$ to release the volatiles into GC/MS for analysis.

\subsubsection{ATR-IR spectroscopy}

A TravelIR attenuated total reflection infrared (ATR-IR) spectrometer, with a SensIR diamond detector crystal, located at the Chemistry Department in the Nebraska Wesleyan University was used to examine as-grown $\{110\}$ and freshly cleaved $\{001\}$ faces of $\mathrm{HgI}_{2}$ crystals grown with low $\bar{M}_{\mathrm{w}}$ polyethylene, as well as a piece of processed low $\overline{\mathrm{M}}_{\mathrm{w}}$ polyethylene sample (see Section 4.5.2). The crystal surface to be examined was placed upside down and in contact with the diamond detector, with a high pressure clamp used to secure the sample. All spectra were collected at $1 \mathrm{~cm}^{-1}$ spectral resolution. A background spectrum was collected for each sample and was subtracted automatically by the software from the spectrum of the sample.

\section{9 $\mathrm{HgI}_{2}$ Frisch Collar Detectors}

One of the objectives of the $\mathrm{HgI}_{2}$ project at $\mathrm{KSU}$ is to grow tetragonal prismatic $\mathrm{HgI}_{2}$ crystals suitable for room-temperature $\gamma$-ray spectrometers. Compared to $\mathrm{CdZnTe}$, which is another material for room-temperature detectors, $\mathrm{HgI}_{2}$ has lower charge carrier mobilities. 
Yet similar to CdZnTe, the hole mobility is significantly lower than the electron mobility, which leads to much lower hole collection efficiency when either $\mathrm{CdZnTe}$ or $\mathrm{HgI}_{2}$ is operated in a planar (two-terminal) detector configuration.

One design that precludes much of the hole contribution to charge induction is the Frisch collar detector design [13-17]. With this detector design, the device can be built such that the contribution of the hole motion towards charge induction is inconsequential. In recent years, CdZnTe Frisch collar detectors have achieved sub-1\% energy resolution, at $662 \mathrm{keV}$, consistently [18]. The simplest Frisch collar detector utilizes a tetragonal prismatic crystal with an aspect ratio of two [19].

The following sections detail the fabrication of a $\mathrm{HgI}_{2}$ Frisch collar detector from a crystal grown with the low molecular weight polyethylene and its characterization. The Frisch collar detector was characterized by comparing the spectra collected by the planar and Frisch collar configurations, employing the Frisch collar detector to collect spectra from various radioactive sources, and examining its charge collection efficiency (CCE).

The spectra and the CCE calculation (Section 5.8), will show that, not only the tetragonal prismatic $\mathrm{HgI}_{2}$ crystals are excellent materials for room-temperature $\gamma$-ray detectors, but the $\mathrm{HgI}_{2}$ Frisch collar detector described in this study also achieved a $1.8 \%$ energy resolution for $662 \mathrm{keV} \gamma$-rays without further electronic corrections, hence, it is considered to be one of the best energy energy resolution for room-temperature $\mathrm{HgI}_{2}$ detetectors $[42,43,45,46]$.

\subsubsection{Fabrication of $\mathrm{HgI}_{2}$ Frisch collar detectors}

As previously mentioned in Sec. 3.1, the tetrahedral layers along the [001] direction are held together by weak van der Waals forces. Therefore, for device fabrication, a $\mathrm{HgI}_{2}$ tetragonal prismatic crystal grown by either low molecular weight polyethylene or ketone could be cleaved easily, parallel to the (001) plane, to a desired size just by using a razor blade. While sectioning the crystal, the aspect ratio was kept at a value between 1.5 and 2.0 [19]. Aquadag E (colloidal carbon) was used as the contact material, applied to the 
crystal ends immediately after the crystal was sectioned, and palladium wires were attached to the carbon contacts for electrical connection. Note that no etching was performed during the planar device fabrication. The tetragonal prismatic shape of the as-grown $\mathrm{HgI}_{2}$ crystals significantly help with the Frisch collar device fabrication, and the fortuitous crystal shape becomes more important for applications requiring numerous detectors (such as arrays).

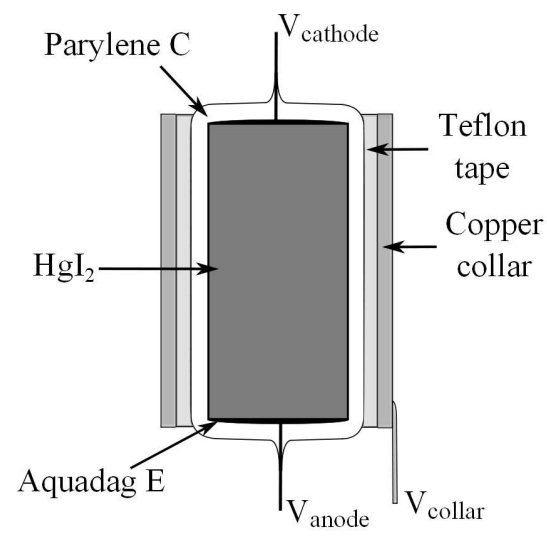

Figure 4.16. $\mathrm{HgI}_{2}$ Frisch collar detector cross section.

Parylene-C, commercially available in many variants [61], was employed as the first dielectric layer. Parylene-C was determined to be the best choice between the commercially available options because of its fast deposition time, high resistivity $\left(8.8 \times 10^{10} \Omega \cdot \mathrm{cm}\right)$, high dielectric breakdown strength $(220 \mathrm{kV} / \mathrm{mm})$, and low dielectric constant $(\kappa \sim 2.95)$ [62]. Moreover, Parylene-C does not react with $\mathrm{HgI}_{2}$, and thus, it was used to encapsulate the planar detector for passivation and stabilization. Parylene coating also reinforced Aquadag contacts for additional mechanical support and easy handling of the device.

Only a thin layer of Parylene-C was deposited as the first insulator layer on the surfaces of the $\mathrm{HgI}_{2}$ planar device, due to the fact that Parylene could not be easily removed, if required, when the deposited layer was thick. Because there is always an optimum dielectric layer thickness for Frisch collar devices [63], other techniques, such as wrapping the device with Teflon tape, was employed to add additional dielectric layer thickness. In this study, $37 \mathrm{mg}$ of Parylene-C dimer was used, resulting in deposition of $50 \mu \mathrm{m}$ Parylene-C as the first layer of dielectric material, followed by two layers of $0.07 \mathrm{~mm}$ thick Teflon 
tape. Afterwards, a thin copper shim was cut to size and used as the Frisch collar, which extended the length of the device and was connected to the device cathode. In this manner, the Frisch collar was held at the cathode potential, and both conductors were grounded.

\subsection{2 $\gamma$-Spectroscopy with $\mathrm{HgI}_{2}$ Frisch collar detectors}

The fabricated $\mathrm{HgI}_{2}$ detector, either in a planar and a Frisch collar configuration, was positioned in an aluminum test box for spectral collection of various radionuclides. The detector within the box was connected to an eV-550 preamplifier and both were placed inside a copper Faraday cage to minimize electronic noise. The measurement system consisted of an amplifier, an oscilloscope, a multi-channel analyzer (MCA), a high voltage supply, a pulse generator, and a personal computer, all positioned outside the Faraday cage. Gammaray sources $\left({ }^{241} \mathrm{Am},{ }^{57} \mathrm{Co},{ }^{133} \mathrm{Ba},{ }^{137} \mathrm{Cs},{ }^{198} \mathrm{Au}\right.$, and $\left.{ }^{60} \mathrm{Co}\right)$ were always placed outside the aluminum test box, in the same position, such that the detector side faced the source.

During the experiment, the temperature and the relative humidity were recorded to be $21^{\circ} \mathrm{C}$ and $75 \%$, respectively. All operating parameters and settings were held constant for all measurements, with the amplifier shaping time set to $1 \mu \mathrm{s}$ and the amplifier gain set to 69X. The detector was biased at $+1500 \mathrm{~V}$, which was applied to the anode while holding the cathode grounded, for more than 16 hours before taking the pulse-height spectra.

\subsubsection{Charge collection efficiency measurements}

The charge collection efficiency (CCE) characterization for the $\mathrm{HgI}_{2}$ Frisch collar detector was performed using a $43.0 \mathrm{~mm}$ long $\mathrm{Pb}$-collimator. The collimator had a $0.6 \mathrm{~mm}$ hole allowing for the detectors to be fully irradiated with a ${ }^{137} \mathrm{Cs}$ gamma ray source over a $0.72 \mathrm{~mm}$ diameter circular area (see Fig. 4.18). The Pb-collimator was mounted on a linear stage with two degrees of freedom, such that the Frisch collar device could be probed by the highly collimated ${ }^{137} \mathrm{Cs}$ gamma-ray source at $0.65 \mathrm{~mm}$ increments along the device length. The Frisch collar detector was placed $8.7 \mathrm{~mm}$ away from the $\mathrm{Pb}$-collimator and was held in place for the entire experiment, while the ${ }^{137} \mathrm{Cs}$ gamma-ray source was aligned 


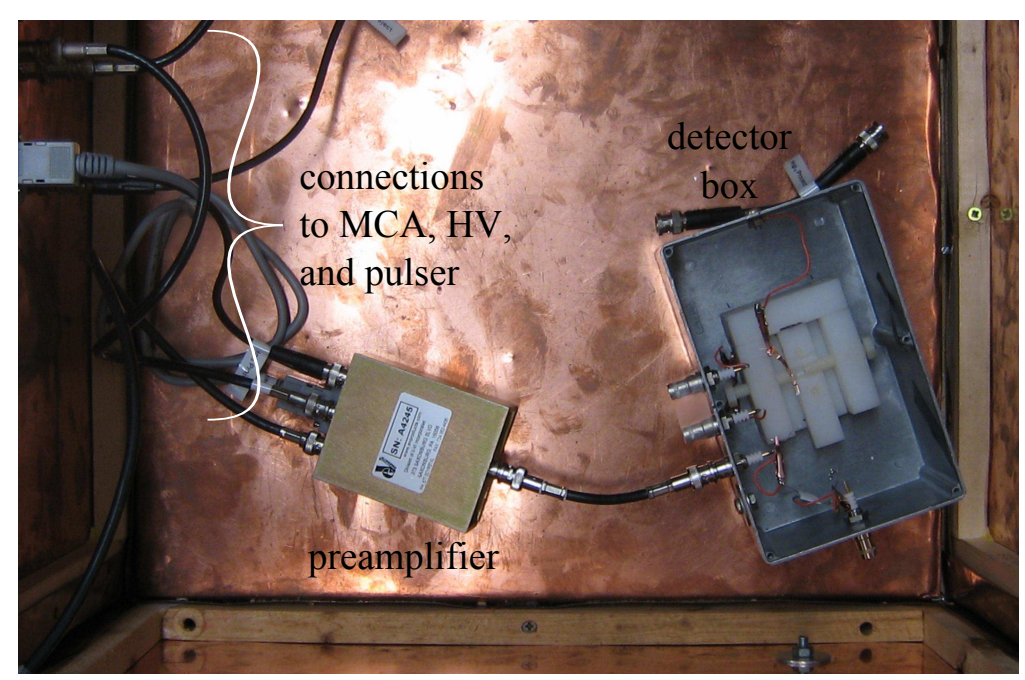

(a)

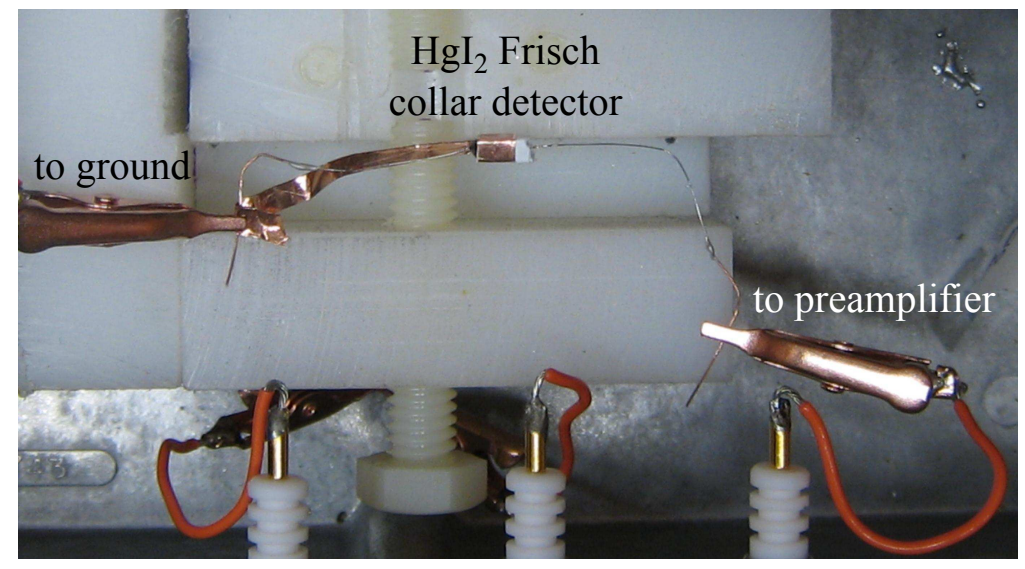

(b)

Figure 4.17. Setup for spectroscopy measurement using a $\mathrm{HgI}_{2}$ Frisch collar detector. (a) The detector box and amplifier were placed inside a Faraday cage. (b) Details of the detector position in the detector box.

with the collimator hole. The detector, gamma-ray source, Pb-collimator, and linear stage were placed inside an aluminum test box.

The $\mathrm{HgI}_{2}$ collar device was then probed with a highly collimated ${ }^{137} \mathrm{Cs}$ gamma-array source to investigate the uniformity of the Frisch collar device's response to high energy gamma-rays. First, the Frisch collar detector and the linear stage were placed inside an aluminum test box, while the detector was connected to an eV-550 preamplifier through an SHV connector. The aluminum test box and the preamplifier were then placed inside a copper Faraday cage, and the preamplifier was connected to a high-voltage supply, ampli- 


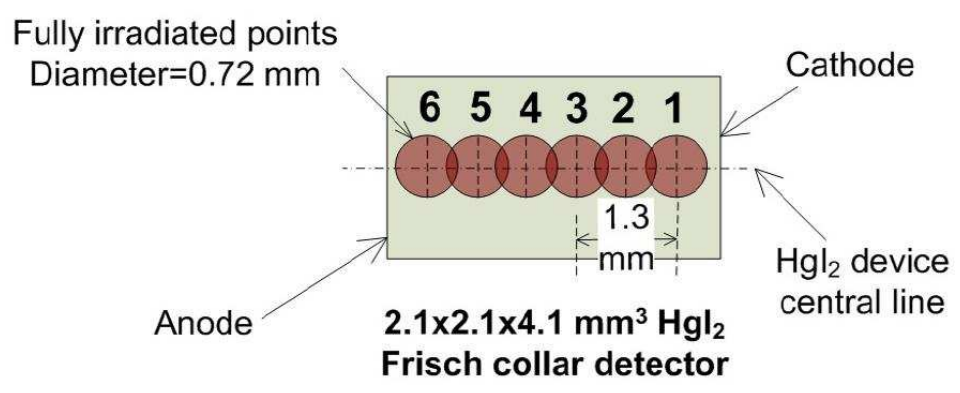

Figure 4.18. The irradiated points on the $2.1 \times 2.1 \times 4.1 \mathrm{~mm}^{3} \mathrm{HgI}_{2}$ Frisch collar device for the CCE measurement. The collimated gamma-ray source on a linear stage allows for irradiating the desired points shown. Here, the $\mathrm{HgI}_{2}$ Frisch collar device was irradiated in increments of $0.65 \mathrm{~mm}$ along the device's central line.

fier and a pulse generator. An oscilloscope, a multichannel analyzer (MCA), and a personal computer were used to monitor and acquire the data.

To conduct the CCE experiment, the device's lateral sides were probed with collimated $662 \mathrm{keV}$ gamma-rays, and a pulse height spectrum was taken (four hours real time at 1500 $\mathrm{V}$ bias for $\mathrm{HgI}_{2}$ detector). The amplifier gain was set to $69 \mathrm{X}$ for the all measurements. Displacement of the source was performed by moving the collimated source using the two-dimensional linear stage in increments of $0.65 \mathrm{~mm}$ along the device length (points 1 through 6) for $\mathrm{HgI}_{2}$ detector as shown on Fig. 4.18 [42]. 


\section{Chapter 5}

\section{Results and Analysis}

The purification and growth results are presented in this chapter. In the discussion on grown crystals, the identification of the crystal faces is as follows. Because the angle between two crystal planes (hence, between two crystal faces) is constant (referred to as the law of constant angle), the position of one crystal face, with respect to the other, identifies the crystal faces. Each crystal face also has a unique "pattern", due in part to its growth mechanism. Therefore, the procedure to determine a face on a grown crystal takes into account the position of the face and the observed pattern (or the lack of it, in the case of $\left.\mathrm{HgI}_{2}\right)$. The three most prominent crystal faces in $\mathrm{HgI}_{2}(001),\{110\}$, and $\{h 0 l\}$, are all identified visually as such.

\subsection{Purification results and characterization by GDMS}

In purification by sublimation in dynamic vacuum, sublimed $\mathrm{HgI}_{2}$ was collected in a Teflon sleeve positioned in the bell jar, as shown in Fig. 4.2. After the third sublimation, the accumulated $\mathrm{HgI}_{2}$ was retrieved, pulverized, and filled in a melting ampoule (Fig. 4.4). The first generation melting ampoule was a one-chamber ampoule, that is, a sealed one with melted charge shown in Fig. 5.1(a). Retrieving the melted charge by breaking open the ampoule risks the chance of including carbonaceous impurities produced during melting 
(Fig. 5.1(b)). Subliming the melted charge in dynamic vacuum by putting opened melting ampoules inside a sublimation ampoule was attempted (Fig. 4.9), however, carbonaceous impurities were often found mixed with $\mathrm{HgI}_{2}$ in the Teflon sleeve. With a two-chamber ampoule design (Fig. 4.4), melting was performed in the first chamber (Fig. 4.5), and the melted charge was moved to the second chamber by sublimation in a compact furnace (Fig. 4.7).

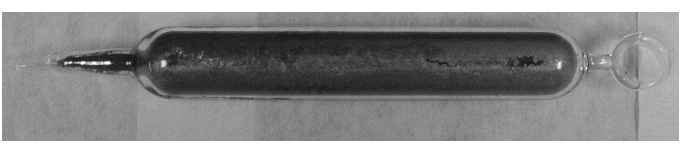

(a)

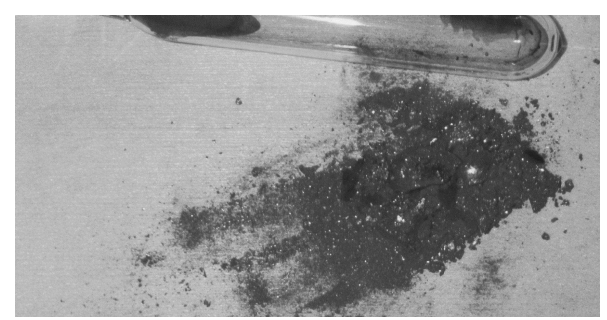

(b)

Figure 5.1. The first generation melting ampoule. (a) One chamber ampoule utilized for melting. (b) Retrieval of melted charge from a one-chamber melting ampoules was first performed by breaking open the ampoule, however, this procedure proved problematic as carboneceous impurities could be included in the retrieved $\mathrm{HgI}_{2}$.

The GDMS trace impurity analysis results are found in Tables 5.1 to 5.4. Table 5.1 shows that the concentrations of impurities found in the stock/starting $\mathrm{HgI}_{2}$ decreased as it underwent the first sublimation in dynamic vacuum (shortened as sublimation for the discussion in this section) and melting (Table 5.1). For a few impurities, their concentrations decreased after the first sublimation, but increased after melting (Table 5.2). This melted sample was retrieved from a purification run performed in a one-chamber melting ampoule, therefore the material was retrieved by breaking the ampoule open (Fig. 5.1(b)). It is believed that some impurities from the carbonaceous impurities were included in the sample. Before being filled into a melting ampoule, $\mathrm{HgI}_{2}$ retrieved from the last step of sublimation also had to be pulverized, a step that could have increased these impurities.

The GDMS results comparing the impurity contents between the second and third sublimation samples are listed in Table 5.3. Elemental impurities examined in Group 1 were also examined in Group 2, however, the impurities that did not change in concentrations after the second and third sublimation runs were not included in the table. The rest of the 
Table 5.1. GDMS results for Group 1 with decreasing impurity concentrations.

\begin{tabular}{|c|c|c|c|}
\hline \multirow[b]{2}{*}{ Element } & \multicolumn{3}{|c|}{$\mathrm{HgI}_{2}$} \\
\hline & $\begin{array}{l}\text { Stock Material } \\
\text { [ppm wt. ] }\end{array}$ & $\begin{array}{c}1^{\text {st }} \text { Sublimation } \\
\text { [ppm wt. ] }\end{array}$ & $\begin{array}{c}\text { Melting } \\
\text { [ ppm wt. ] }\end{array}$ \\
\hline $\mathrm{N}$ & $\sim 200$ & $\sim 25$ & $\sim 20$ \\
\hline $\mathrm{O}$ & $\sim 20$ & $\sim 2$ & $\sim 2$ \\
\hline $\mathrm{Al}$ & 0.48 & 0.35 & 0.28 \\
\hline $\mathrm{Si}$ & 2 & 1.8 & 0.38 \\
\hline $\mathrm{P}$ & 0.02 & $<0.005$ & $<0.005$ \\
\hline $\mathrm{S}$ & 8 & 0.35 & 0.35 \\
\hline $\mathrm{Cl}$ & 35 & 14 & 2 \\
\hline $\mathrm{Ca}$ & 0.5 & $<0.05$ & $<0.05$ \\
\hline $\mathrm{Ti}$ & 0.75 & 0.17 & 0.03 \\
\hline $\mathrm{Cr}$ & 0.02 & 0.02 & $<0.01$ \\
\hline $\mathrm{Fe}$ & 1.7 & 0.28 & 0.28 \\
\hline $\mathrm{Ni}$ & 0.06 & $<0.01$ & $<0.01$ \\
\hline $\mathrm{Br}$ & 1.8 & 1.2 & 0.45 \\
\hline $\mathrm{Ag}$ & $\leq 30$ & $\leq 2$ & $\leq 0.7$ \\
\hline In & 0.15 & $<0.005$ & $<0.005$ \\
\hline $\mathrm{Sn}$ & 0.2 & $<0.01$ & $<0.01$ \\
\hline $\mathrm{Sb}$ & 0.75 & $<0.01$ & $<0.01$ \\
\hline W & 0.04 & $<0.01$ & $<0.01$ \\
\hline $\mathrm{Pb}$ & 0.12 & $<0.05$ & $<0.05$ \\
\hline
\end{tabular}

Table 5.2. GDMS results for Group 1 with increasing impurity concentrations.

\begin{tabular}{cccc}
\hline & \multicolumn{3}{c}{$\mathrm{HgI}_{2}$} \\
\cline { 2 - 4 } Element & $\begin{array}{c}\text { Stock Material } \\
\text { [ ppm wt. ] }\end{array}$ & $\begin{array}{c}1^{\text {st }} \text { Sublimation } \\
\text { [ ppm wt. ] }\end{array}$ & $\begin{array}{c}\text { Melting } \\
\text { [ ppm wt. ] }\end{array}$ \\
\hline $\mathrm{B}$ & 0.02 & 0.18 & 0.04 \\
$\mathrm{C}$ & 12 & 2 & 15 \\
$\mathrm{Na}$ & 0.15 & 0.05 & 3 \\
$\mathrm{Mg}$ & 0.04 & $<0.005$ & 0.01 \\
$\mathrm{~K}$ & 20 & $<0.05$ & 0.15 \\
$\mathrm{As}$ & 0.18 & 0.22 & 0.08 \\
\hline
\end{tabular}

elemental impurities also decreased in concentrations within at least one order of magnitude. The only impurity increasing in concentration was that of sodium (Na), although the concentrations after the second and third sublimation runs were still along the same order of magnitude. Therefore, the overall results for Group 2 shows that successive sublimation runs did remove impurities more than one order of magnitude in concentrations.

The GDMS results comparing the impurity contents between samples of $\mathrm{HgI}_{2}$ before and after the polyethylene pre-growth processing step (Group 3) are listed in Table 5.4. Again, the elemental impurities that did not change in concentrations were left out from the 
table. The rest of the elemental impurities increased in concentrations, most notably carbon (C), which increased about twenty-fold. The only slightly decreasing concentration was that of $\mathrm{Na}$. Therefore, the overall results for Group 3 show that a few elemental impurities increased in concentration during the pre-growth processing step.

Table 5.3. GDMS results for Group 2.

\begin{tabular}{ccc}
\hline & \multicolumn{2}{c}{$\mathrm{HgI}_{2}$} \\
\cline { 2 - 3 } Element & $\begin{array}{c}\text { 2nd Sublimation } \\
{[\mathrm{ppm} w \mathrm{wt}]}\end{array}$ & $\begin{array}{c}\text { 3rd Sublimation } \\
{[\mathrm{ppm} w \mathrm{wt}]}\end{array}$ \\
\hline $\mathrm{C}$ & $<30$ & $<10$ \\
$\mathrm{Na}$ & 0.1 & 0.4 \\
$\mathrm{Mg}$ & 0.01 & $<0.001$ \\
$\mathrm{Al}$ & 0.07 & $<0.005$ \\
$\mathrm{Si}$ & 0.2 & $<0.005$ \\
$\mathrm{Cl}$ & 560 & 200 \\
$\mathrm{Fe}$ & 0.04 & 0.01 \\
$\mathrm{As}$ & $\leq 5$ & $\leq 1$ \\
$\mathrm{Br}$ & 7 & 4 \\
$\mathrm{~Pb}$ & 0.2 & 0.08 \\
\hline
\end{tabular}

Table 5.4. GDMS results for Group 3.

\begin{tabular}{ccc}
\hline & \multicolumn{2}{c}{$\mathrm{HgI}_{2}$} \\
\cline { 2 - 3 } Element & $\begin{array}{c}\text { Melting-Sublimation } \\
{[\text { ppm wt }]}\end{array}$ & $\begin{array}{c}\text { PE-added } \\
{[\text { ppm wt ] }}\end{array}$ \\
\hline $\mathrm{C}$ & $<5$ & $<100$ \\
$\mathrm{~N}$ & $<100$ & $<400$ \\
$\mathrm{O}$ & $<60$ & $<200$ \\
$\mathrm{Na}$ & 0.03 & 0.02 \\
$\mathrm{Si}$ & 0.01 & 0.1 \\
$\mathrm{~S}$ & 0.01 & 0.04 \\
$\mathrm{Cl}$ & 60 & 130 \\
$\mathrm{As}$ & $\leq 0.5$ & $\leq 1$ \\
$\mathrm{Br}$ & 2 & 6 \\
$\mathrm{Bi}$ & 0.1 & 1 \\
\hline
\end{tabular}




\subsection{Growth With No Additive}

In this section, observations on how the change in source mass affected $\mathrm{HgI}_{2}$ crystallization in the horizontal furnace without any additive are described in Section 5.2.1, while the effects of residual pressure on $\mathrm{HgI}_{2}$ crystallization are described in Section 5.2.2.

\subsubsection{The effect of source mass}

Fig. 5.2(a) shows the growth result for $39.3 \mathrm{~g}$ of starting $\mathrm{HgI}_{2}$, and 5.3(a) shows the growth results with half the amount of $\mathrm{HgI}_{2}(40 \mathrm{~g})$. For both growth runs, the source temperature was set at $\mathrm{T}=100^{\circ} \mathrm{C}$, with a temperature gradient of $\Delta \mathrm{T}=20^{\circ} \mathrm{C}$, and both growth ampoules were evacuated to $\sim 10^{-4}$ Torr. The same growth period of five days was employed. Finally, each growth ampoule was placed in a different growth furnace, both with similar axial temperature profiles.

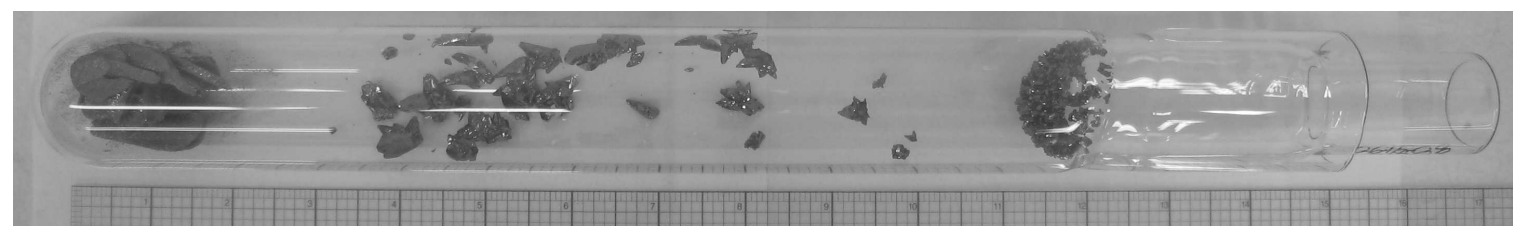

(a)

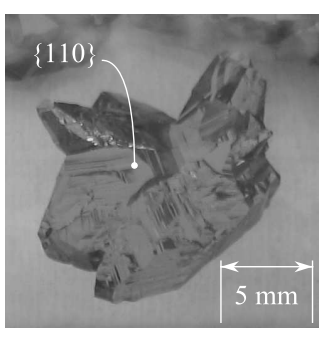

(b)

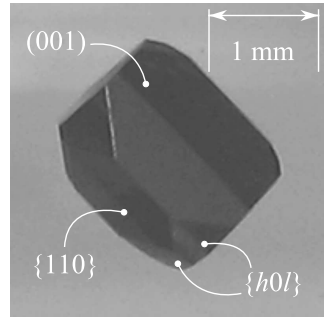

(c)

Figure 5.2. (a) $\mathrm{HgI}_{2}$ growth results with no additive, with $80 \mathrm{~g}$ of starting $\mathrm{HgI}_{2}$. (b) $\mathrm{A}$ cyclic twin $\mathrm{HgI}_{2}$ crystal, where $\{110\}$ crystal faces were observed with square-shaped growth layers. (c) A platelet-shaped single crystal, where (001), $\{110\}$, and $\{h 0 l\}$ could be observed .

$\mathrm{HgI}_{2}$ crystals grown without any additive were usually twinned, as shown in Figs. 5.2(b) and 5.3(b). Single crystals, such as in Fig. 5.2(c), could also be found, however seldomly and in smaller sizes. Furthermore, crystal faces $\{110\}$ were always found in either twinned 
or single crystals, while additionally the (001) and $\{$ h0l $\}$ faces were also observed in single crystals.

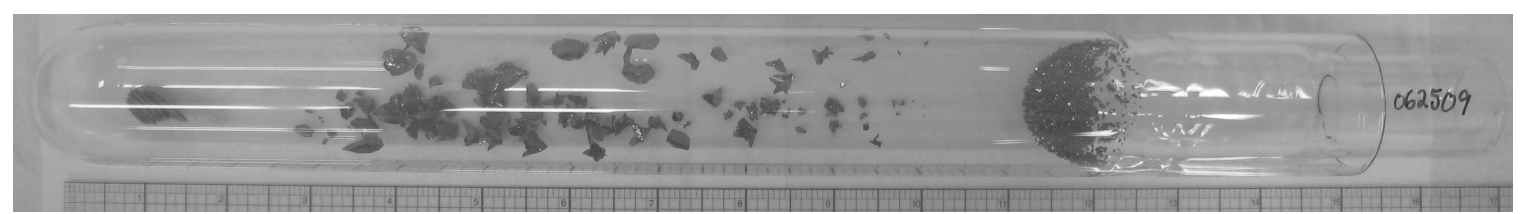

(a)

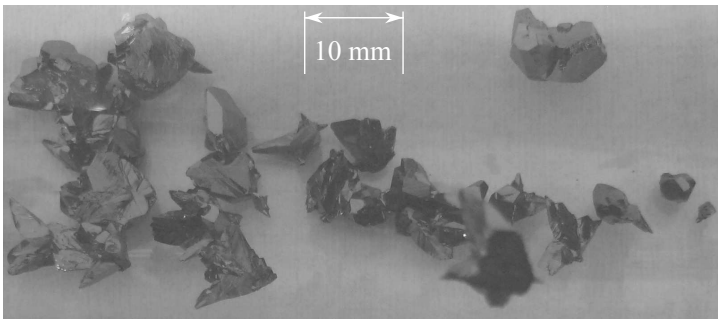

(b)

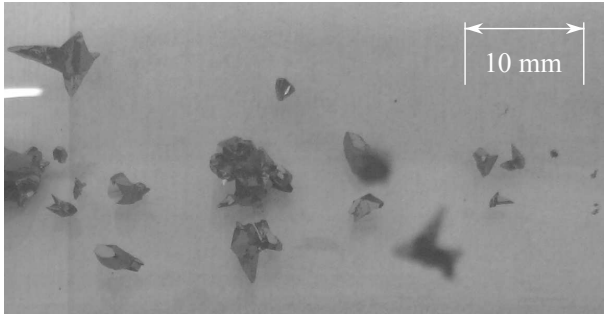

(c)

Figure 5.3. (a) $\mathrm{HgI}_{2}$ growth results with no additive with $40 \mathrm{~g}$ of starting $\mathrm{HgI}_{2}$. (b) Twinned $\mathrm{HgI}_{2}$ crystals found at the beginning of the crystallization zone. (c) Smaller twinned crystals found near the end of the crystallization zone.

Approximately the same crystallization zone lengths were observed in both ampoules in Figs. 5.2(a) and 5.3(a). Crystals in the beginning of the crystallization zone were larger than the ones growing near the end of the zone. This observation shows that the growth nutrients were incorporated more readily into surfaces closer to the growth source. The same explanation addresses the reason for more severe cases of twinning at the beginning of the crystallization zone than toward the end.

\subsubsection{The effect of residual pressure}

After a growth period of five days with source temperature $\mathrm{T}=100^{\circ} \mathrm{C}$, temperature gradient $\Delta \mathrm{T}=20^{\circ} \mathrm{C}$, and starting pressure at room temperature $\mathrm{P}(\mathrm{RT}) \sim 4.2$ Torr, both single and twinned crystals grew in the crystallization zone, with sizes much smaller compared to the crystals obtained in growth with $\mathrm{P}(\mathrm{RT}) \sim 10^{-4}$ Torr (see Figs. $5.2-5.3$ ). As also observed in Section 5.2.1, more nucleation sites were found at the beginning of the crystallization zone than in the end of the zone. 


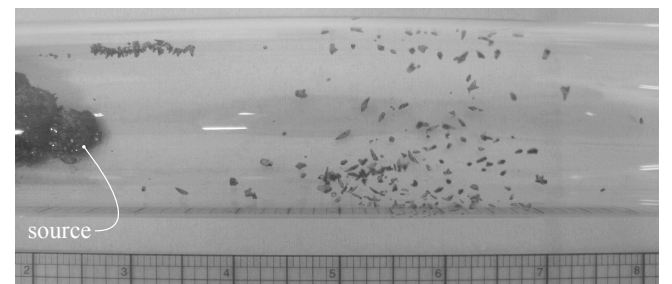

(a)

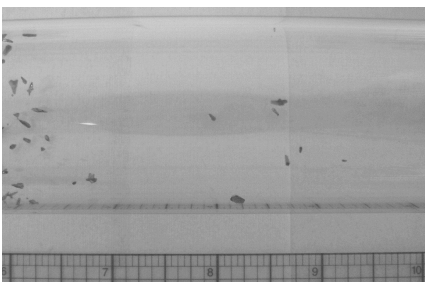

(b)

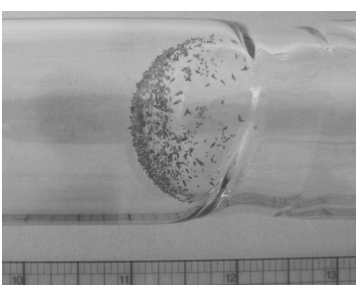

(c)

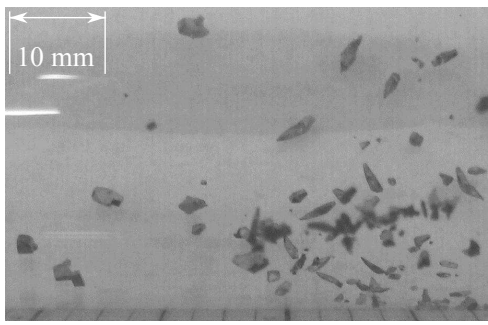

(d)

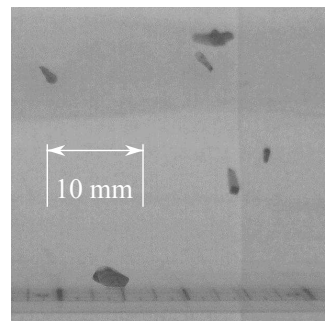

(e)

Figure 5.4. $\mathrm{HgI}_{2}$ growth results with no addition and with a starting pressure of $\sim 4.2$ Torr at room temperature. $\mathrm{T}=100^{\circ} \mathrm{C}, \Delta \mathrm{T}=20^{\circ} \mathrm{C}$. (a) Small crystals grew in the crystallization zone after a growth period of five days. (b) Sparse nucleation sites occurred at the end of the crystallization zone. (c) Crystallites grew on the plug. (d) Details of some crystals in (a). (e) Details of crystals in (b).

Subsequently, another growth run was done with the same ampoule, subjected to $\mathrm{T}=$ $150^{\circ} \mathrm{C}$ and $\Delta \mathrm{T}=10^{\circ} \mathrm{C}$. Before the start of the growth run, the crystals had to be sublimed back to the source end. This resublimation of materials was performed by placing the ampoule in reverse (i.e., the source end was placed at the entrance of the furnace) in a two-zone growth furnace, with temperature of both zones set at $100^{\circ} \mathrm{C}$.

Figs. 5.5(a) and 5.5(c) show the growth results minutes after the ampoule was retrieved from the furnace, in which the crystal color was yellow. As anticipated, only $\beta$ $\mathrm{HgI}_{2}$ formed at temperatures above the phase transformation temperature of approximately $130^{\circ} \mathrm{C}$. While the crystals were cooling down to room temperature, the crystal color transitioned from yellow to red, as shown in Figs. 5.5(b) and 5.5(c).

$\mathrm{HgI}_{2}$ is known to have several crystal phases in a variety of temperature and pressure ranges $[37,38,64]$. These phases are sometimes named according to their corresponding or apparent colors: the $\alpha$-phase is called the red modification, while the $\beta$-phase (the phase above the phase transformation temperature of $130^{\circ} \mathrm{C}$ ) is called the yellow modifi- 


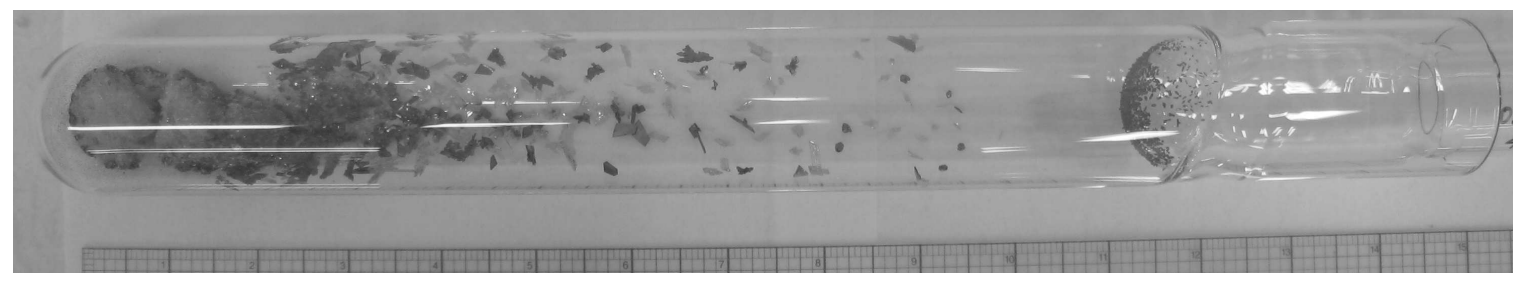

(a)

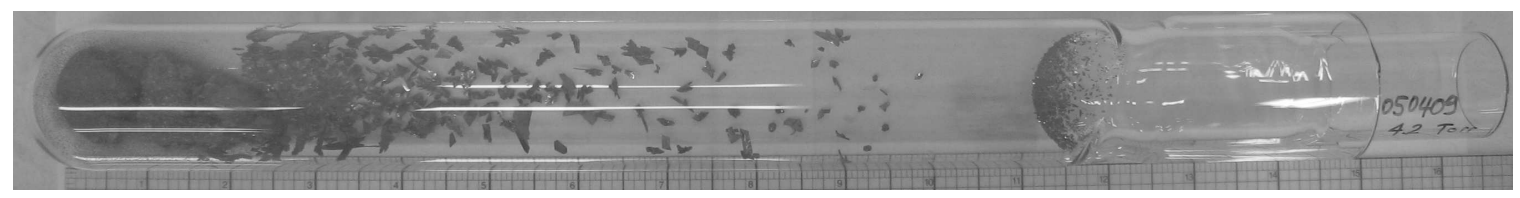

(b)

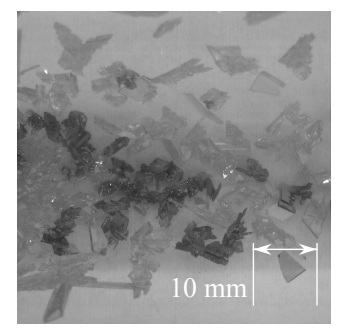

(c)

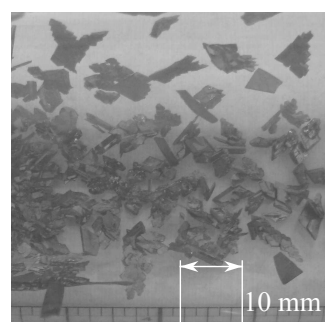

(d)

Figure 5.5. $\mathrm{HgI}_{2}$ growth results with no additive and starting pressure of $\sim 4.2$ Torr at room temperature, $\mathrm{T}=150^{\circ} \mathrm{C}, \Delta \mathrm{T}=10^{\circ} \mathrm{C}$, after a growth period of five days. (a) Growth results after retrieval from the furnace and during cooling down. (b) Growth results in (a) after cooling down. (c) Thin yellow platelets in the growth ampoule, after being retrieved from the furnace. (d) The yellow crystals turned red after cooling down.

cation. These color-designated labels, however, can be misleading, because there can exist different crystal structures and/or symmetrys for the same apparent crystal color.

There is a metastable yellow phase, which can be found growing alongside red crystals at room temperature or at temperatures below $130^{\circ}$, and the high-temperature yellow phase, which is the $\beta$-phase. According to [37], the crystal structure for the metastable yellow is orthorhombic, with a space group designation of $C m c 2_{1}$ (no. 36 in [57]), while the crystal structure for $\mathrm{HgI}_{2} \beta$-phase is monoclinic, with a space group designation of $P 2_{1}$ (no. 4 in [57]). Red crystals and yellow crystals are also found in high pressure conditions [38], therefore information on temperature and/or pressure influencing the formation of the crystals must be added to the color designations. The crystals observed in Fig. 5.5 were obviously high temperature yellow $\beta-\mathrm{HgI}_{2}$ because of the temperatures the ampoule was 
subjected to.

\subsection{Growth With Pre-Growth Polyethylene Processing}

In this section, $\mathrm{HgI}_{2}$ growth that required pre-growth polyethylene processing is described. Growth with spectrophotometric grade polyethylene is described in Section 5.3.1, while growth with low $\overline{\mathrm{M}}_{\mathrm{w}}$ polyethylene and ultra-high $\overline{\mathrm{M}}_{\mathrm{w}}$ polyethylene are described in Sections 5.3.2 and 5.3.3, respectively.

\subsubsection{Growth with spectrophotometric grade polyethylene}

Results of growth with spectrophotometric grade polyethylene are analyzed using the method of factorial analysis (Section 5.3.1.2), which are explained using the aids of a normal probability plot (Section 5.3.1.2.1) and a Lenth's plot (Section 5.3.1.2.2).

\subsubsection{Growth results}

An example of the results of the pre-growth polyethylene processing step, condensing $\mathrm{HgI}_{2}$ on the plug at the cold end, is shown in Fig. 5.6(a). The condensing $\mathrm{HgI}_{2}$ was retrieved by breaking open the ampoule and placing it in a new ampoule for growth. The starting spectrophotometric grade polyethylene did not look melted, as shown in Fig. 5.6(b).

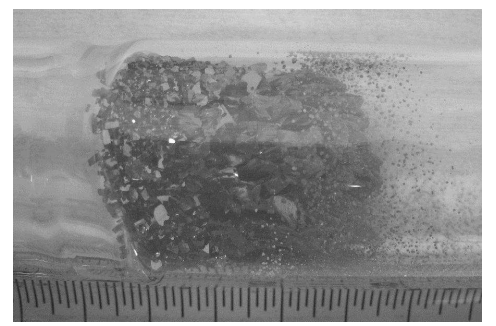

(a)

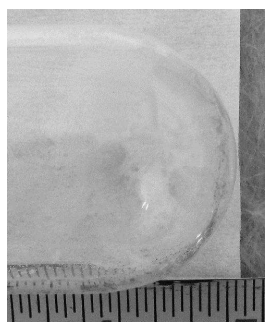

(b)

Figure 5.6. (a) Condensing $\mathrm{HgI}_{2}$ on the plug after a pre-growth polyethylene processing step, with spectrophotometric grade polyethylene. (b) Spectrophotometric grade polyethylene in the round bottom end of the ampoule, after a pre-growth polyethylene processing step. 
Table 5.5 shows the growth ampoules with spectrophotometric grade polyethylene and the respective growth conditions, each condition based on three growth factors: $\mathbf{T}=$ source zone temperature $\left({ }^{\circ} \mathrm{C}\right), \mathbf{G}=\Delta \mathrm{T}\left({ }^{\circ} \mathrm{C}\right)$, the temperature gradient between the source and crystallization zone set temperature points, and $\mathbf{M}=$ additive amount (in wt- $\%$ with respect to the weight of starting $\mathrm{HgI}_{2}$ ). As Table 5.5 shows, ampoules GPE100407 and GPE101107 were used for repeat growth runs. In several growth ampoules, no growth was found in the crystallization zone; hence, the (crystallization) zone length for the respective test condition is zero inches. Analysis by the factorial method is in Section 5.3.1.2.

Table 5.5. Factorial analysis of growth with spectrophotometric grade polyethylene.

\begin{tabular}{ccccccc}
\hline $\begin{array}{c}\text { Test } \\
\text { Condition }\end{array}$ & $\begin{array}{c}\mathbf{T} \\
\left({ }^{\circ} \mathbf{C}\right)\end{array}$ & $\begin{array}{c}\mathbf{G} \\
\left({ }^{\circ} \mathbf{C}\right)\end{array}$ & $\begin{array}{c}\mathbf{M} \\
(\mathbf{w t}-\%)\end{array}$ & Ampoule & Labels & $\begin{array}{c}\text { Zone Length } \\
\text { (inches) }\end{array}$ \\
\hline 1 & 90 & 10 & 1 & GPE100407 & $(1)$ & 0 \\
2 & 100 & 10 & 1 & GPE081007 & $T$ & 3.75 \\
3 & 90 & 20 & 1 & GPE122007 & $G$ & 6.5 \\
4 & 100 & 20 & 1 & GPE100407 & $T G$ & 6.5 \\
5 & 90 & 10 & 2 & GPE101107 & $M$ & 0 \\
6 & 100 & 10 & 2 & GEP101107 & $T M$ & 0 \\
7 & 90 & 20 & 2 & GPE101107 & $G M$ & 5.5 \\
8 & 100 & 20 & 2 & GPE092907 & $T G M$ & 7.5 \\
\hline
\end{tabular}

Crystals in Figs. 5.8(a) - 5.8(e) correspond to the growth ampoules in Figs. 5.7(a) - 5.7(e), respectively. Comparing these crystals with those grown with no additive (Figs. 5.2(a) - 5.3(c)) shows that, along with single crystals, twinned crystals also grew. Unlike the crystals grown with no additive, however, the shape of the crystals grown with spectrophotometric polyethylene was bounded by flat faces, resulting in a tetragonal prismatic crystal habit. Both single and twinned crystals grown with spectrophotometric polyethylene had this habit. 


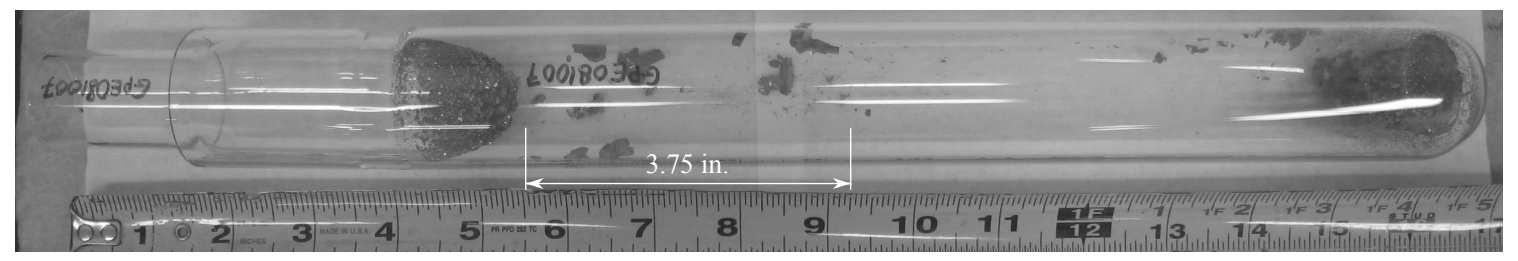

(a) $\mathrm{T}=100^{\circ} \mathrm{C}, \Delta \mathrm{T}=10^{\circ} \mathrm{C}, \mathrm{M}=1 \mathrm{wt}-\%$

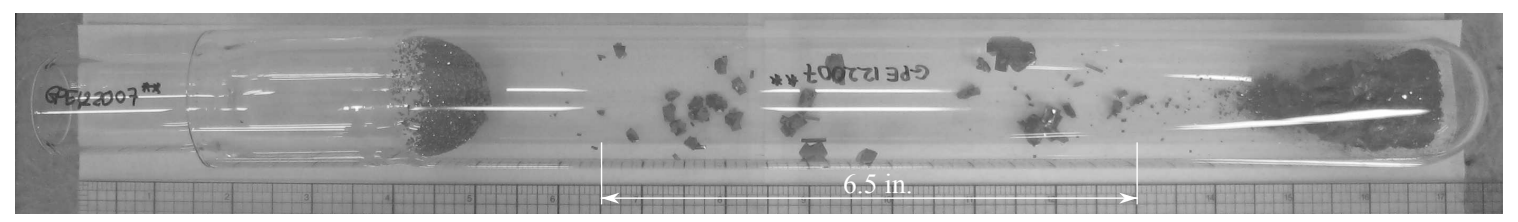

(b) $\mathrm{T}=90^{\circ} \mathrm{C}, \Delta \mathrm{T}=20^{\circ} \mathrm{C}, \mathrm{M}=1 \mathrm{wt}-\%$

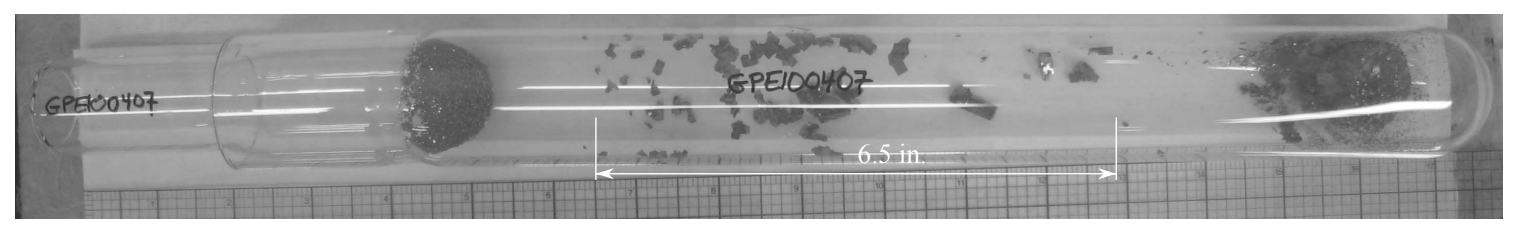

(c) $\mathrm{T}=100^{\circ} \mathrm{C}, \Delta \mathrm{T}=20^{\circ} \mathrm{C}, \mathrm{M}=1 \mathrm{wt}-\%$

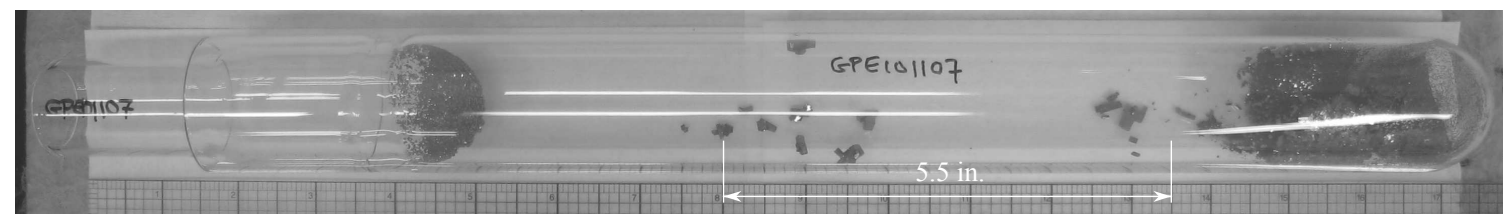

(d) $\mathrm{T}=100^{\circ} \mathrm{C}, \Delta \mathrm{T}=10^{\circ} \mathrm{C}, \mathrm{M}=2 \mathrm{wt}-\%$

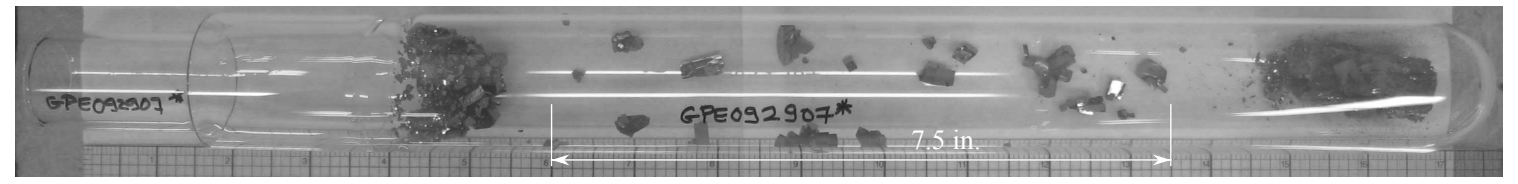

(e) $\mathrm{T}=100^{\circ} \mathrm{C}, \Delta \mathrm{T}=20^{\circ} \mathrm{C}, \mathrm{M}=2$ wt $-\%$

Figure 5.7. Growth results with spectrophotometric grade polyethylene under different growth conditions (see captions). The crystallization growth length in each ampoule is as noted. These values are evaluated by factorial analysis (see Tables 5.6 and 5.7). 


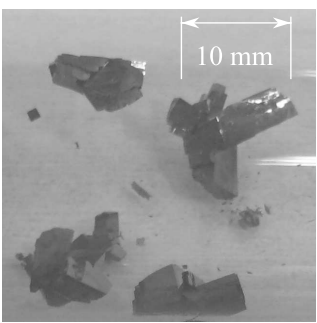

(a)

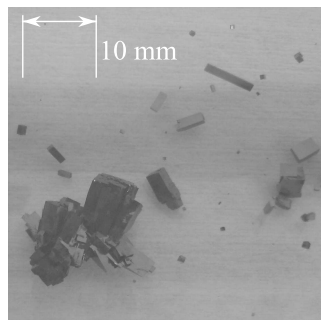

(b)

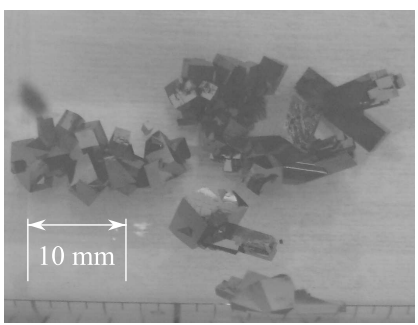

(c)

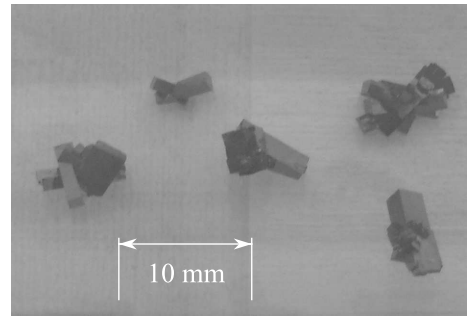

(d)

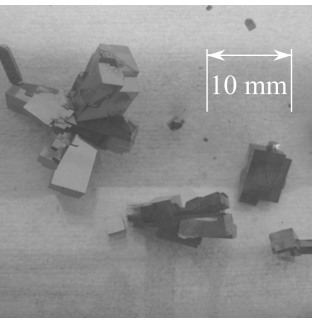

(e)

Figure 5.8. $\mathrm{HgI}_{2}$ crystals grown with spectrophotometric grade polyethylene. Crystals in Figs. 5.8(a) - 5.8(e) correspond to the growth ampoules in Figs. 5.7(a) - 5.7(e), respectively. 


\subsubsection{Factorial analysis}

The $2^{3}$ factorial method (see Appendix A) was used to determine the effects of the growth factors or the variables (see Table 4.3) on the observable, the crystallization zone length. Using this method, three variables were taken into account, and each variable was evaluated at two different values (a minimum and maximum values). Therefore, $2^{3}$ or eight possible growth conditions correspond to eight combinations of the values of the growth factors. The effects are determined by Yates method as explained in Appendix A.

In Table 5.5, each test or growth condition has a label that corresponds to a growth factor or a combination of growth factors evaluated at the maxima. For example, test condition 4, with $\mathbf{T}$ and $\mathbf{G}$ at their maxima, is labeled $T G$, while test condition 1, with each growth factor at its minimum, is labeled (1). The crystallization zone lengths indicated in Table 5.5 are also indicated on Figs. 5.7(a) - 5.7(e). The calculated effects (Table 5.6) are analyzed by a normal probability plot, which is supplemented by a Lenth's plot. Appendix A offers both explanations on how to obtain a normal probability plot and a Lenth's plot. The effects as calculated in Table 5.6 were reordered and their corresponding probability $p$ and $z$-score were calculated and listed in Table 5.7.

Table 5.6. The observables and effect calculations.

\begin{tabular}{|c|c|c|c|c|c|c|c|c|}
\hline $\begin{array}{c}\mathbf{T} \\
\left({ }^{\circ} \mathbf{C}\right)\end{array}$ & $\begin{array}{c}\mathbf{G} \\
\left({ }^{\circ} \mathbf{C}\right)\end{array}$ & $\begin{array}{c}\mathbf{M} \\
\left({ }^{\circ} \mathbf{C}\right)\end{array}$ & $\begin{array}{l}\text { Zone Length } \\
\text { (inches) }\end{array}$ & $\mathbf{X}$ & $\mathbf{Y}$ & $\mathbf{Z}$ & Effect & Label \\
\hline- & - & - & 0 & 3.75 & 16.75 & 29.75 & 3.71875 & (1) \\
\hline+ & - & - & 3.75 & 13 & 13 & 5.75 & 1.4375 & $T$ \\
\hline - & + & - & 6.5 & 0 & 3.75 & 22.25 & 5.5625 & $G$ \\
\hline+ & + & - & 6.5 & 13 & 2 & -1.75 & -0.4375 & $T G$ \\
\hline- & - & + & 0 & 3.75 & 9.25 & -3.75 & -0.9375 & $M$ \\
\hline+ & - & + & 0 & 0 & 13 & -1.75 & -0.4375 & $T M$ \\
\hline - & + & + & 5.5 & 0 & -3.75 & 3.75 & 0.9375 & $G M$ \\
\hline+ & + & + & 7.5 & 2 & 2 & 5.75 & 1.4375 & $T G M$ \\
\hline
\end{tabular}


Table 5.7. Probability values and z-scores of the effects of growth factors on the crystallization zone length.

\begin{tabular}{ccccc}
$\mathbf{i}$ & $\mathbf{p}$ & $\mathbf{z}$ & $\mathbf{E f f e c t}$ & Label \\
\hline 1 & 0.063 & -1.534 & -0.9375 & $M$ \\
2 & 0.188 & -0.887 & -0.4375 & $T G$ \\
3 & 0.313 & -0.489 & -0.4375 & $T M$ \\
4 & 0.438 & -0.157 & 0.9375 & $G M$ \\
5 & 0.563 & 0.157 & 1.4375 & $T G M$ \\
6 & 0.688 & 0.489 & 1.4375 & $T$ \\
7 & 0.813 & 0.887 & 3.71875 & $(1)$ \\
8 & 0.938 & 1.534 & 5.5625 & $G$ \\
\hline
\end{tabular}

\subsection{Normal probability plot}

The normal probability plot is produced by plotting the z-score against its corresponding estimated effect (Table 5.7). When data distribution follows normal distribution, the data presented in a normal probability plot can be fitted in a straight line. Therefore, any estimated effect that falls around a straight line is considered a statistical effect. Any data points that lie far from the straight line, however, are considered to truly influence the experiment.

The effects were fitted to a straight line following the least-square fit line method. The rule of thumb for determining the position of the fit line is to fit the data to 25 th to 75 th percentiles. The data presented in Fig. 5.9 were fitted with a least-squares fit line for five effects ( $T G, T M, G M, T G M, T)$, chosen because the overall $\mathrm{R}^{2}$ value for the fit line was lower than the fit line for six data points, including $T G M$. As a result, $M$ and $G$ were far from the straight line, and therefore these effects (the change in additive's mass and temperature gradient) were real (non-stochastic) effects that influenced the length of the crystallization zone. 


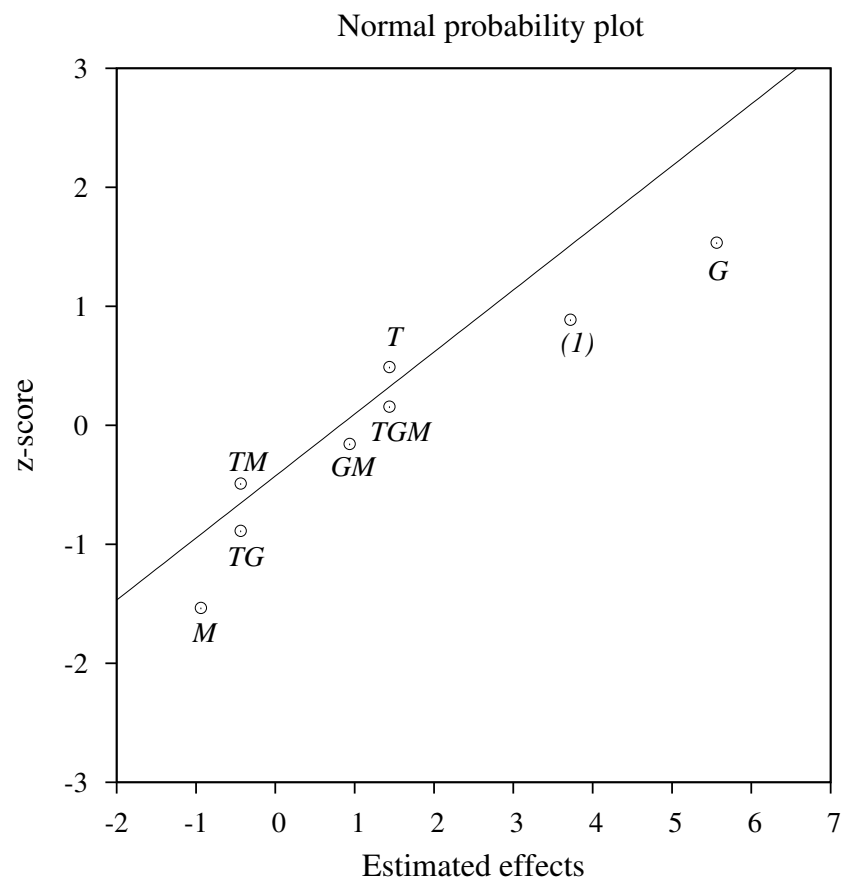

Figure 5.9. The normal probability plot, which is the plot of z-scores vs. the estimated effects. The analysis is done by fitting the data within the 25 th to 75 th percentiles to the least-squares linear fit. The effects that are far from the linear fit are considered real effects. In this case, $G$ and $M$ are real effects. $T G M$ is not a real effect, since all three growth factors were altered.

\subsection{Lenth's plot}

A Lenth's plot can supplement the information obtained from the normal probabilty plot by plotting the contrasts (which is $\mathrm{Z}$ in Table 5.7) against the respective estimated effects. The effects were compared to the margin of error (ME) and simultaneous margin or error (SME) values. These values were plotted as straight lines parallel to the $\mathrm{x}$-axis on either side of the X-axis. Effects that are beyond those values or beyond either $\pm \mathbf{M E}$ are considered possible contributing effects, while the ones beyond either \pm SME are considered strong or real effects.

According to the Fig. 5.10, the effect $T G M$ is beyond $+\mathbf{M E}$, but, because all three growth factors were at their higher set points, the effect did not have any meaning. However, the effect $G$ is beyond $+\mathbf{S M E}$, indicating that the change in temperature gradient 


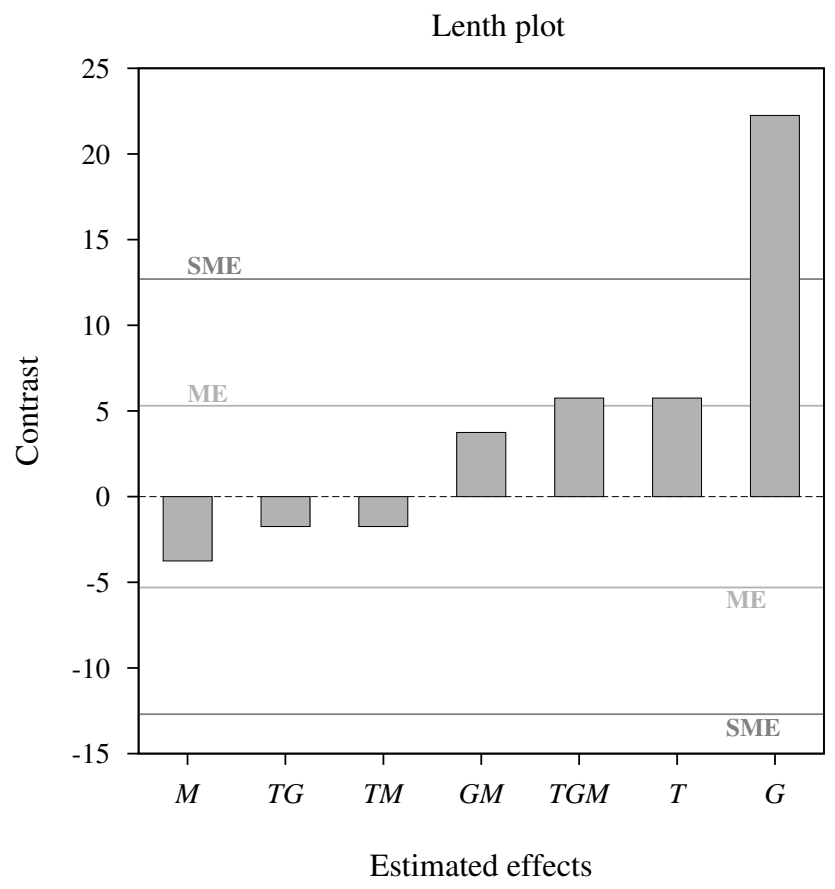

Figure 5.10. Lenth's plot, which is the plot of the contrasts vs. the estimated effects. Shown also are the $\pm \mathbf{M E}$ and \pm SME lines. The effects with contrasts that are beyond \pm SME are considered real. In this case $G$ (temperature gradient) is a real effect that influenced the length of the crystallization zone.

produced a real effect or a real change in the crystallization length. Hence, the Lenth's plot supports the finding of the normal probability plot.

\subsubsection{Growth with low $\bar{M}_{\mathrm{w}}$ polyethylene}

Fig. 5.11 shows an example of a pre-growth polyethylene processing ampoule with low $\overline{\mathrm{M}}_{\mathrm{w}}$ polyethylene, which was typically in solidified melt condition after this pre-growth procedure. As described previously, the $\mathrm{HgI}_{2}$ condensed on the plug was retrieved by breaking the ampoule open, taking care in avoiding inclusion of melted polyethylene. The retrieved $\mathrm{HgI}_{2}$ was loaded into a new ampoule for growth.

After the growth with spectrophotometric polyethylene, growth runs with polyethylene of different molecular weights were conducted, specifically low $\overline{\mathrm{M}}_{\mathrm{w}}$ and ultra high $\overline{\mathrm{M}}_{\mathrm{w}}$. The growth runs with low $\overline{\mathbf{M}}_{\mathrm{w}}$ polyethylene were subjected to the same growth conditions 
Figure 5.11. An ampoule after a pre-growth polyethylene processing step with low $\overline{\mathrm{M}}_{\mathrm{w}}$ polyethylene. Condensed $\mathrm{HgI}_{2}$ was found on the plug, while all of the starting low $\overline{\mathrm{M}}_{\mathrm{w}}$ polyethylene melted.

as those with spectrophotometric grade polyethylene (Table 5.6). Results of the growth runs are shown in Fig. 5.13 and Fig. 5.12 shows the crystals of the growth with low $\overline{\mathrm{M}}_{\mathrm{w}}$ polyethylene. Twinning of crystals (for example, Fig. 5.12(a) and 5.12(d)) is still observed; however, more single crystals with tetragonal prismatic shapes are found as well. These single crystals grow with the [001] axis parallel (for example, Fig. 5.12(b)) or perpendicular to the ampoule's wall (for example, Fig. 5.12(c)).

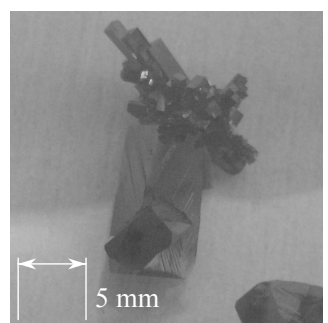

(a)

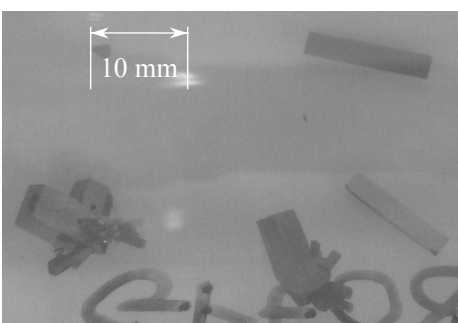

(b)

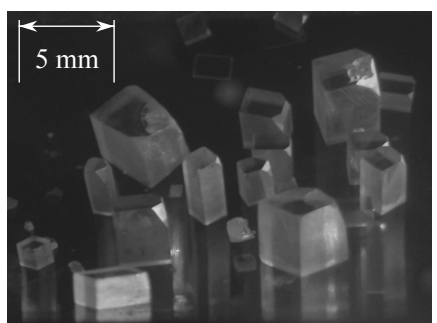

(c)

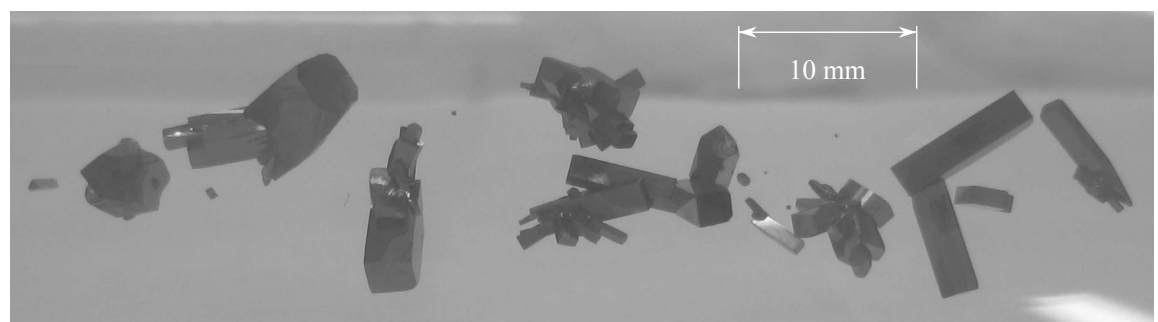

(d)

Figure 5.12. $\mathrm{HgI}_{2}$ crystals grown with low $\overline{\mathrm{M}}_{\mathrm{w}}$ polyethylene. Crystals in Figs. 5.12(a)5.12(d) are crystals from the ampoules in Figs. 5.13(a)-5.13(d), respectively. 


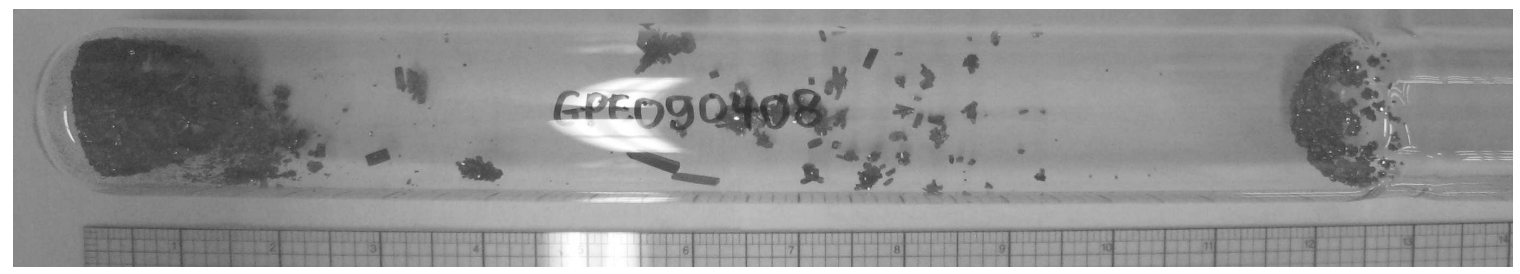

(a) $\mathrm{T}=90^{\circ} \mathrm{C}, \Delta \mathrm{T}=20^{\circ} \mathrm{C}, \mathrm{M}=1 \mathrm{wt}-\%$

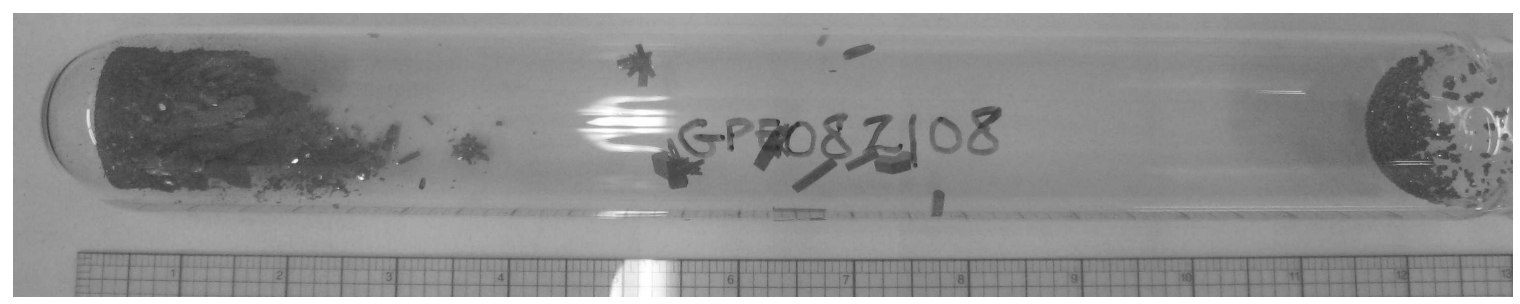

(b) $\mathrm{T}=90^{\circ} \mathrm{C}, \Delta \mathrm{T}=20^{\circ} \mathrm{C}, \mathrm{M}=2 \mathrm{wt}-\%$

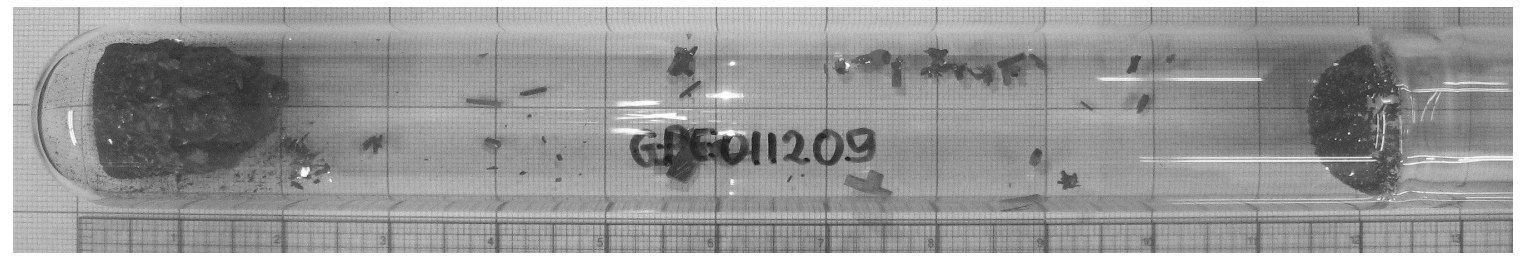

(c) $\mathrm{T}=100^{\circ} \mathrm{C}, \Delta \mathrm{T}=10^{\circ} \mathrm{C}, \mathrm{M}=2 \mathrm{wt}-\%$

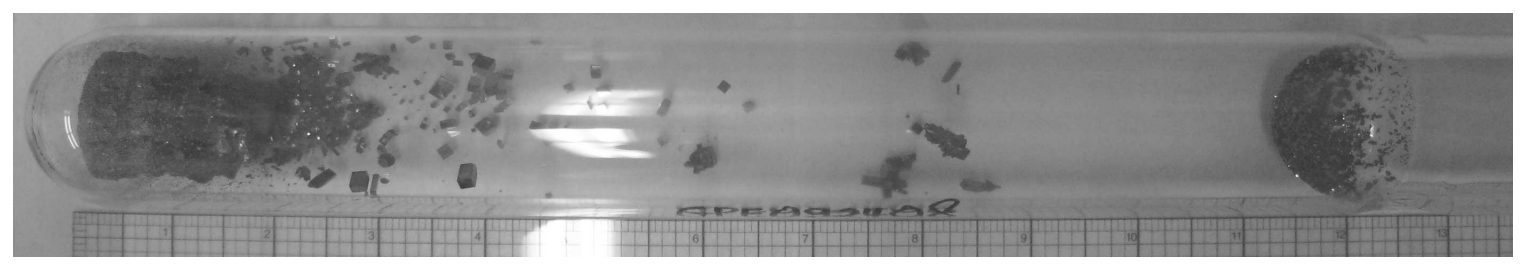

(d) $\mathrm{T}=100^{\circ} \mathrm{C}, \Delta \mathrm{T}=20^{\circ} \mathrm{C}, \mathrm{M}=2 \mathrm{wt}-\%$

Figure 5.13. Growth with low $\bar{M}_{\mathrm{w}}$ polyethylene, showing the growth results under different growth conditions (see captions). 


\subsubsection{Growth with ultra high $\bar{M}_{\mathrm{w}}$ polyethylene}

The results of the pre-growth processing step with ultra high $\overline{\mathrm{M}}_{\mathrm{w}}$ polyethylene are shown in Fig. 5.14. As in the results of previous pre-growth processing step, sublimed $\mathrm{HgI}_{2}$ condensed on the plug at the end of the crystallization zone. The ultra high $\overline{\mathrm{M}}_{\mathrm{w}}$ polyethylene left at the round bottom end of the ampoule, however, did not seem to decompose or evaporate.

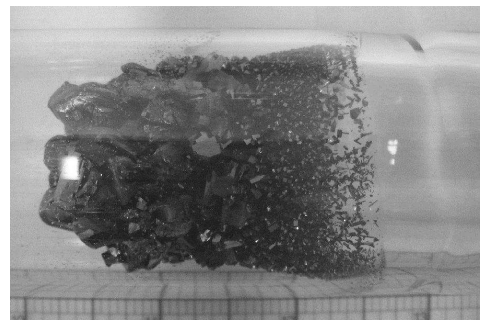

(a)

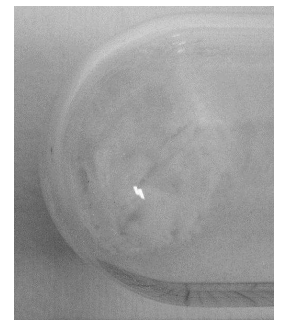

(b)

Figure 5.14. (a) Sublimed $\mathrm{HgI}_{2}$ condensed on the plug at the end of the crystallization zone. (b) The ultra high $\overline{\mathrm{M}}_{\mathrm{w}}$ polyethylene left at the round bottom end of the ampoule did not seem to decompose or evaporate.

The result of the growth run with ultrahigh $\overline{\mathrm{M}}_{\mathrm{w}}$ polyethylene is in Fig. 5.16. Most of the larger crystals in the crystallization zone were twinned and retained the crystallographic faces seen in the crystals grown without any organic addition, but the crystals growing on the plug, although smaller, formed in the prismatic tetragonal shapes (Fig 5.16(b)).

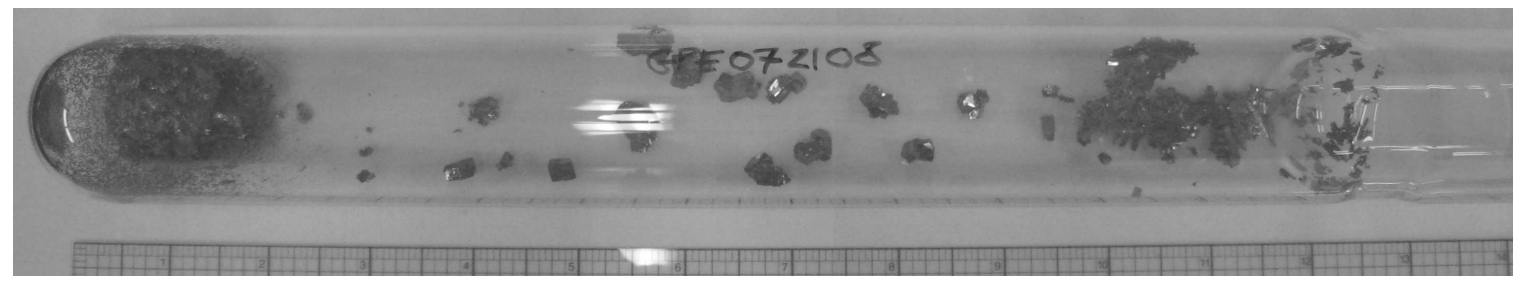

(a)

Figure 5.15. Growth with ultra high $\overline{\mathrm{M}}_{\mathrm{w}}$ polyethylene, with $\mathrm{T}=100^{\circ} \mathrm{C}, \Delta \mathrm{T}=20^{\circ} \mathrm{C}$, and $\mathrm{M}=2 \mathrm{wt}-\%$. 


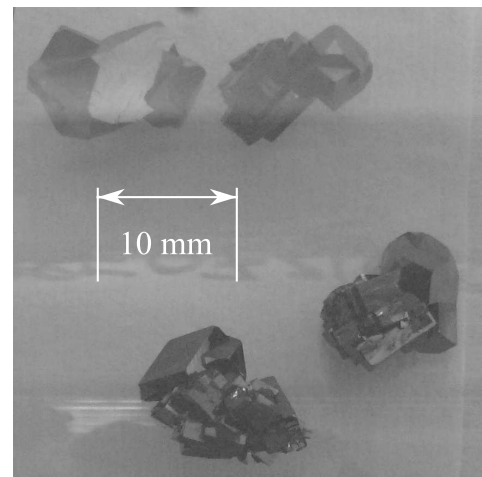

(a)

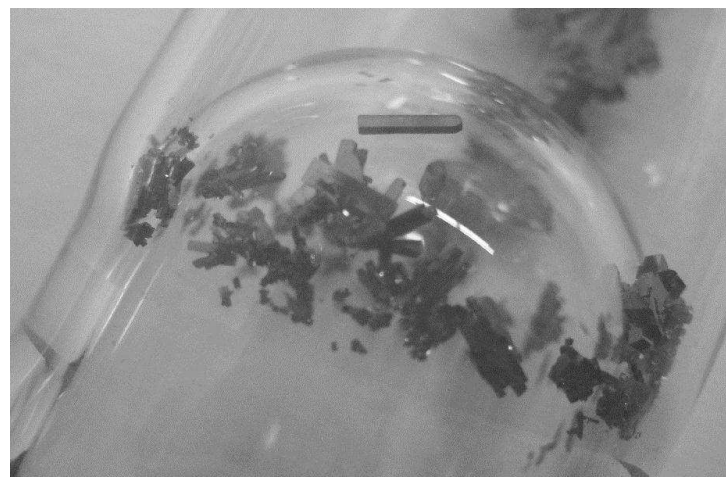

(b)

Figure 5.16. (a) Most of the crystals in the crystallization zone were twinned. (b) Smaller crystals on the plug had prismatic tetragonal shapes (most of the crystals on the plug were inadvertently dislodged upon cooling).

\subsubsection{Growth ampoule observations}

Inspections on the growth ampoules were conducted to further investigate the differences observed in the growth results when different polyethylene types were employed. For all growth runs, the same $\mathrm{HgI}_{2}$ purification procedure (Section 4.1) was followed, and for growth runs proceeded by a pre-growth polyethylene processing step, the same ampoule preparation and sealing procedures were followed (Section 4.5.2). The only difference, therefore, was the type of polyethylene.

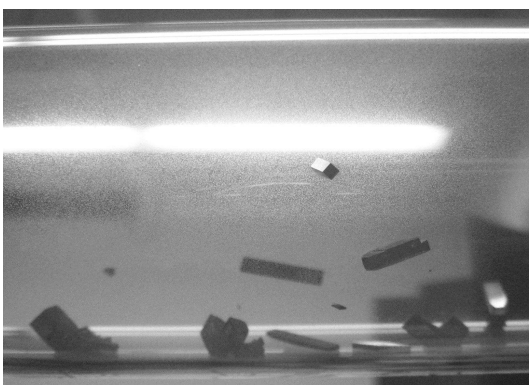

(a)

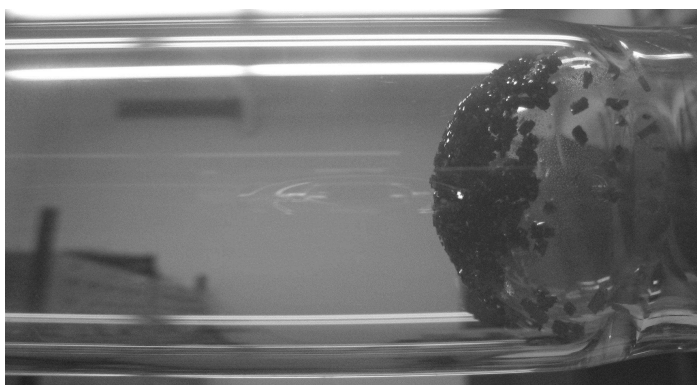

(b)

Figure 5.17. Observations on a growth ampoule with low $\bar{M}_{w}$ polyethylene. (a) The haziness of ampoule wall at the beginning and the middle of the crystallization zone, as it was seen against the light, was caused by tiny droplets of polymer condensation. (b) Nearing the end of the crystallization zone, there was little to no polymer condensation, except for droplets of polymer condensation found on the plug.

Observations on a growth ampoule with low $\overline{\mathrm{M}}_{\mathrm{w}}$ polyethylene are shown in Fig. 5.17. 


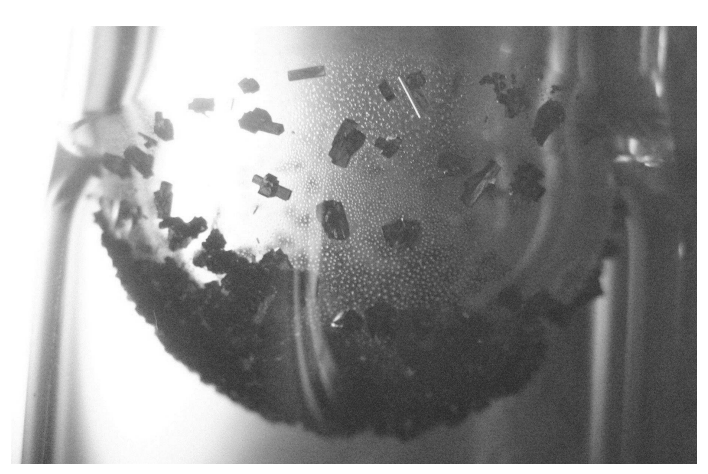

(a)

Figure 5.18. Details of the droplets of polymer condensation found on the plug.

The haziness of ampoule wall at the beginning and the middle of the crystallization zone, as it was seen against the light (Fig. 5.17(a)), was caused by tiny droplets of polymer condensation. Nearing the end of the crystallization zone there was little to no polymer condensation, except for the droplets found on the plug (Figs. 5.17(b) and 5.18(a)). These polymer droplets were not found in either the growth with spectrophotometric grade or ultra high $\overline{\mathrm{M}}_{\mathrm{w}}$ polyethylene.

From the point of view of the growth procedures, there are several possibilities for the origin of the droplets, considering that the procedure for the pre-growth processing step includes placing the starting $\mathrm{HgI}_{2}$ and one type of polyethylene powder together in an ampoule (Section 4.5.2). An experiment was done to determine whether low $\overline{\mathrm{M}}_{\mathrm{w}}$ polyethylene, when subjected to vacuum and elevated temperatures, could produce volatiles in the absence of $\mathrm{HgI}_{2}$. In this experiment, a small glass vial filled with low $\overline{\mathrm{M}}_{\mathrm{w}}$ polyethylene was placed in an ampoule and sealed using the same sealing procedure employed previously. The ampoule was then placed in a two-zone furnace, with Zone 1 temperature set at $230^{\circ} \mathrm{C}$ and Zone 2 at $150^{\circ} \mathrm{C}$ (the temperatures used in the pre-growth polyethylene processing stage - Section 4.5.1), for five days.

Clear droplets could be seen condensing on the plug during the time the ampoule was in the furnace. After the ampoule was removed from the furnace, these clear droplets and the melted polyethylene left behind in the ampoule both turned white upon cooling down (Fig. 5.19). Therefore, in the absence of $\mathrm{HgI}_{2}$, low $\overline{\mathrm{M}}_{\mathrm{w}}$ polyethylene, when subjected to 
vacuum and elevated temperatures, indeed produced volatiles. Whether these volatiles were low $\overline{\mathrm{M}}_{\mathrm{w}}$ polyethylene molecules, shorter chain molecules produced from the scission of longer molecules, or impurities in the starting polyethylene, remain to be explored. These volatile molecules, however, appeared to have condensed on the sublimed $\mathrm{HgI}_{2}$, and hence, were present at the beginning of the growth run, and thus, might affect the change in crystal morphology.

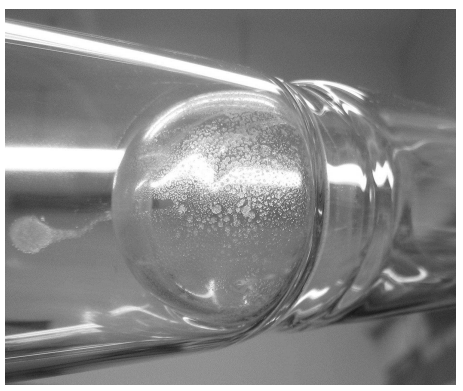

(a)

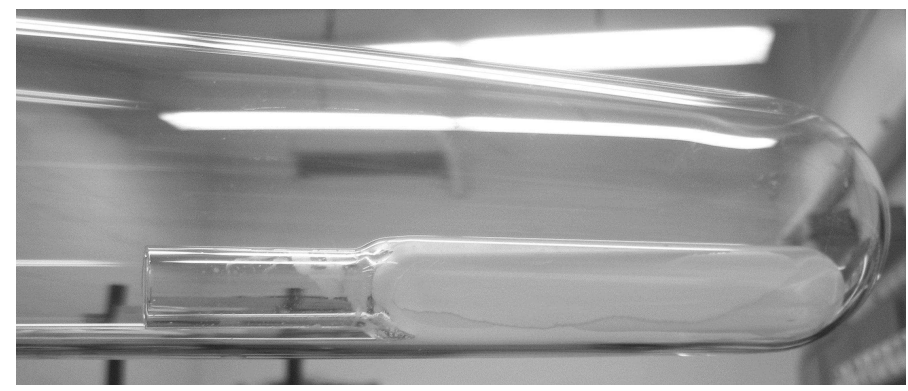

(b)

Figure 5.19. Vaporization of low polyethylene in the absence of $\mathrm{HgI}_{2}$. (a) Droplets of polymer were found on the plug. (b) Melted $\mathrm{HgI}_{2}$, which was clear during the time the ampoule was in the furnace, turned white upon cooling down.

Polyethylene, especially high density polyethylene, is generally stable when heated up to $300^{\circ} \mathrm{C}$ in inert atmosphere, where it begins to degrade through chain breakage and crosslinking. As the temperature increases to $500^{\circ} \mathrm{C}$, still in inert atmosphere, the polyethylene undergoes pyrolysis, which means that it thermally breaks down, to lower molecular weight compounds, such as alkanes, alkenes, and dienes. In the presence of oxygen, the polyethylene undergoes thermo-oxidation starting at $200^{\circ} \mathrm{C}$, which results in oxygen containing low molecular weight compounds, such as water, aldehydes, and ketones [65].

The temperatures used for the pre-growth polyethylene processing step were lower than the onset temperature for pyrolysis, however, they were near the onset temperatures for thermo-oxidation. Care was taken during ampoule preparation and sealing to remove oxygen from the ampoules as much as possible. The pre-growth polyethylene process (Section 4.5.1), as well as the vaporization of the low molecular weight polyethylene described in this section, and the entire growth runs in this study, were completed in vacuum $\left(\sim 10^{-4}\right.$ Torr), with several $\mathrm{N}_{2}$ gas backfill runs prior to ampoule sealing, in order to remove air 
(hence, $\mathrm{O}_{2}$ ) from the ampoules (Section 4.2.3).

Nevertheless, there are factors in the growth procedures, in which oxygen presence cannot be excluded. For example, natural gas $-\mathrm{O}_{2}$ torch was used to seal the melting, pregrowth processing and growth ampoules, at temperatures above $800^{\circ} \mathrm{C}$, higher than both the pyrolysis and thermo-oxidation onset temperatures. However, because the ampoules employed in the growth without any additive were also sealed with the same method, even if or when oxygen was still present, the level was such that it might not permit the creation of a lot of volatile low molecular weight compounds. The polyethylene powders used in the growth runs were stored in their original containers (in a clean room, but in air and not in inert atmosphere), and used as-received, without further purification. Therefore, impurities were present in the starting polyethylene powders.

To determine possible influences of mixing $\mathrm{HgI}_{2}$ and polyethylene starting materials on $\mathrm{HgI}_{2}$ growth shapes, a growth run with separated starting materials are examined in Section 5.3.5. Second, to directly determine that the volatiles produced from heating the low molecular weight polyethylene influenced $\mathrm{HgI}_{2}$ growth shapes, several growth runs with $\mathrm{HgI}_{2}$ mixed with various amounts of low molecular weight polyethylene, without the pre-growth processing step (Section 4.5.1), were described in Section 5.4. Third, to determine the types of the volatile molecules, direct analysis of the volatiles using thermal desorption (TD)-GC/MS is described in Section 5.7.1.

\subsubsection{Growth with unmixed $\mathrm{HgI}_{2}$ and polyethylene source}

To determine whether mixing the starting materials had any consequence on the production of the volatiles, an experiment with separated or unmixed $\mathrm{HgI}_{2}$ and low $\overline{\mathrm{M}}_{\mathrm{w}}$ polyethylene was performed. Based on the observations that volatiles were produced from heating low $\overline{\mathrm{M}}_{\mathrm{w}}$ polyethylene in the absence of $\mathrm{HgI}_{2}$ in vacuum, it was anticipated that the separation of the starting $\mathrm{HgI}_{2}$ and low $\overline{\mathrm{M}}_{\mathrm{w}}$ polyethylene in two small vials (as described in Section 4.5.2) would produce the same type of crystallization as in the growth runs with mixed starting materials. Fig. 5.20(a) shows the vials before the pre-growth processing 
stage. After $\mathrm{HgI}_{2}$ was transported to and condensed at the cold end (Fig. 5.20(b)), it was retrieved by breaking open the ampoule, and then it was placed in a new ampoule for growth.

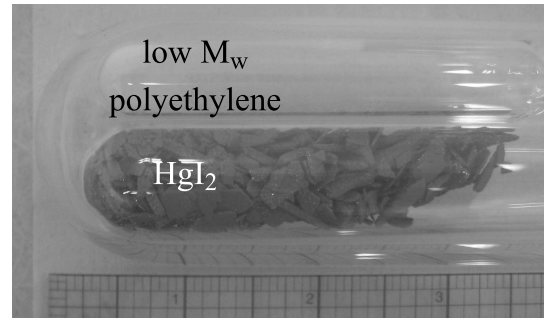

(a)

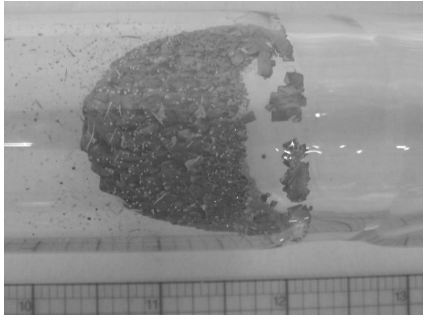

(b)

Figure 5.20. Pre-growth processing for a growth runs with separated starting materials (purified $\mathrm{HgI}_{2}$ and low $\overline{\mathrm{M}}_{\mathrm{w}}$ polyethylene). (a) Before the pre-growth processing step: the starting materials in their own glass vials. (b) After the pre-growth processing step: $\mathrm{HgI}_{2}$ condensing on the plug at the end of the crystallization zone.

Fig. 5.21 shows the growth ampoule, where in the crystallization zone more nucleations appears to have occurred compared to the crystallization with mixed sources (for example, Fig. 5.13), as evidenced by small crystallites dispersed in between the bigger crystals (Fig. 5.21(b)). A detailed inspection of one of the twinned crystal reveals the $\{110\}$ and the (001) faces (Fig. 5.21(d)). Twinning of tetragonal prismatic crystals is also observed (Fig. $5.21(d))$. 


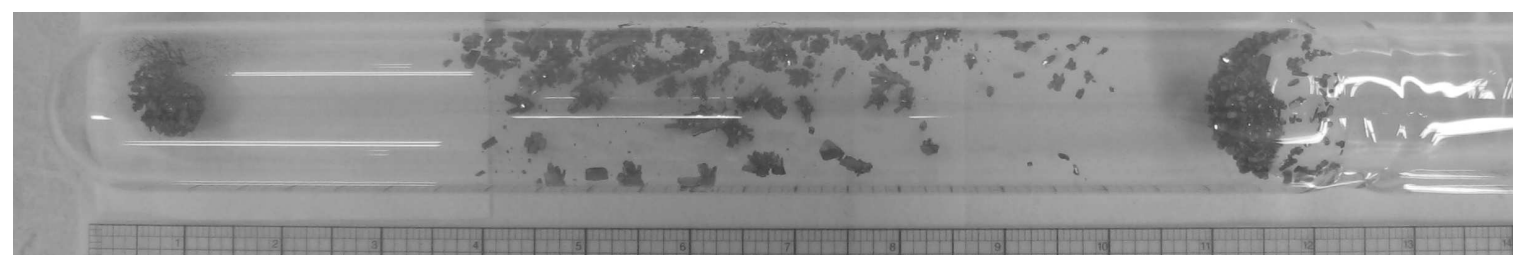

(a)

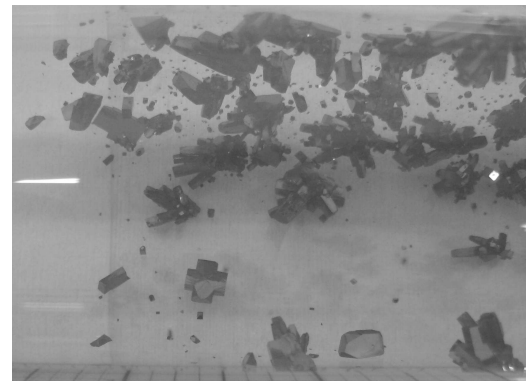

(b)

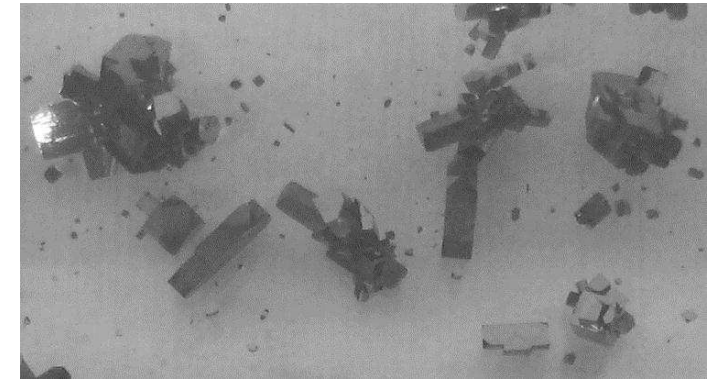

(c)

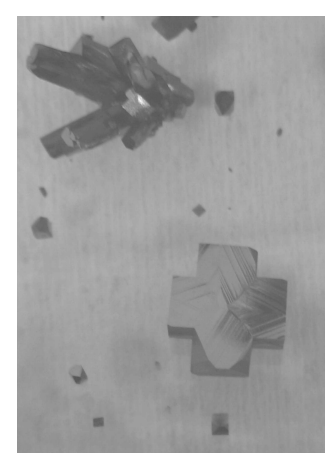

(d)

Figure 5.21. Growth results with separated source materials (purified $\mathrm{HgI}_{2}$ and low $\overline{\mathrm{M}}_{\mathrm{w}}$ polyethylene). (a) The growth ampoule, where (b) an observation on the crystallization zone shows more nucleations occurred as evidenced by crystallites dispersed in between larger crystals. (c) Twinning of tetragonal prismatic crystals also occurred. 


\subsection{Growth Without Pre-Growth Polyethylene Processing}

The growth results with low $\overline{\mathrm{M}}_{\mathrm{w}}$ polyethylene, with the starting materials either mixed (Section 5.3.2) or separated (Section 5.3.5), along with the observations of the growth ampoules (Section 5.3.4), indicate the presence of organic volatiles in the growth ampoules. As also observed when only the low $\overline{\mathbf{M}}_{\mathrm{w}}$ polyethylene was subjected to a high temperature (Fig. 5.19), organic volatiles were still produced without being in contact with $\mathrm{HgI}_{2}$, suggesting that these organic volatiles originated from the starting low $\overline{\mathrm{M}}_{\mathrm{w}}$ polyethylene. At present, how these volatiles were produced is not as consequential as what these compounds were, and knowing what chemicals were produced could eventually answer how they affected the crystallization of $\mathrm{HgI}_{2}$ in the horizontal growth.

A more pronounced change in $\mathrm{HgI}_{2}$ crystal morphology obtained when the low $\overline{\mathrm{M}}_{\mathrm{w}}$ polyethylene was utilized (as compared to when other types of polyethylene were employed), along with the presence of the organic volatiles when the polyethylene was subjected to higher temperatures (even in the absence of $\mathrm{HgI}_{2}$ ), implies that the pre-growth polyethylene processing step (Section 4.5.1) may not be necessary. Additional growth runs with mixed starting materials, and with none going through the pre-growth processing step, were completed to ascertain whether a change in crystal morphology would still occur. Three growth ampoules were prepared with three different amounts of low $\overline{\mathrm{M}}_{\mathrm{w}}$ polyethylene: 0.02 wt- $\%$ (with $80 \mathrm{~g}$ of $\mathrm{HgI}_{2}$ ), $0.5 \mathrm{wt}-\%$ (with $50 \mathrm{~g}$ of $\mathrm{HgI}_{2}$ ) and $5 \mathrm{wt}-\%$

(with $50 \mathrm{~g}$ of $\left.\mathrm{HgI}_{2}\right)$. After each ampoule was sealed under vacuum $\left(\sim 10^{-4}\right.$ Torr), it was placed in a two-zone growth furnace with the source zone temperature set at $100^{\circ} \mathrm{C}$ and the crystallization zone temperature set at $80^{\circ} \mathrm{C}$, for a growth time of five days .

Figs. $5.22-5.24$ show the three ampoules after growth and their corresponding crystals. In the growth with $0.02 \mathrm{wt}-\%$ polyethylene, most of the crystals had the shape of those grown without polyethylene, as indicated by the $\{110\}$ faces showing the squareshaped growth layers, similar to the $\{110\}$ faces in crystals grown without polyethylene (Figs. 5.22(b) and (c)). These crystals grew mostly in the beginning of the crystallization zone. Near the end of the crystallization zone, prismatic tetragonal crystals grew, though 
not as numerous (Figs. 5.22(d) and (e)). In the growth with $0.5 \mathrm{wt}-\%$ polyethylene, more crystals growing with tetragonal prismatic shapes were found (again, the crystal faces were us as indicators of the shapes), however, their apparent sizes were smaller than the crystals grown with 0.02 wt-\% polyethylene. Much fewer crystals, all appeared to acquire the tetragonal prismatic crystal shapes, grew in the growth with 5 wt- $\%$ polyethylene. Twinning, which occurred in every growth, was still found, but single prismatic crystals were also found, as well as a yellow $\mathrm{HgI}_{2}$ platelet that remained yellow even upon cooling down.

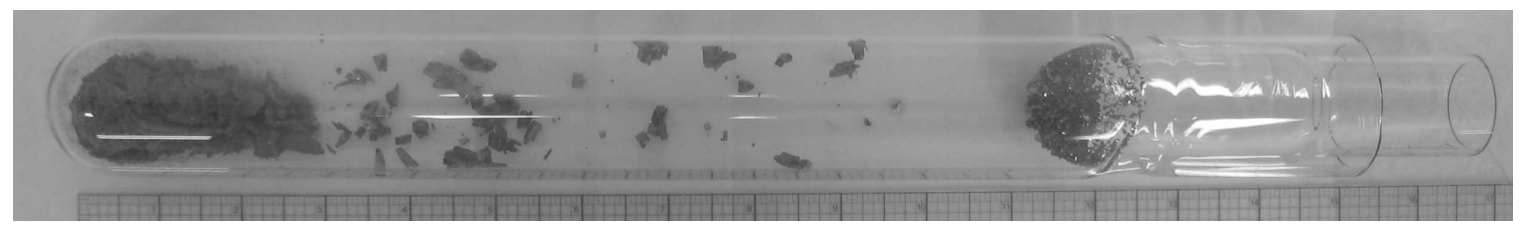

(a)

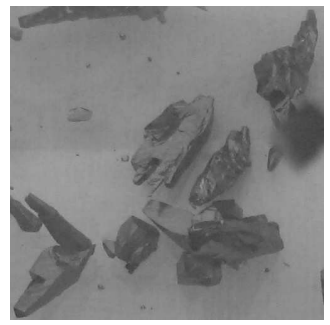

(b)

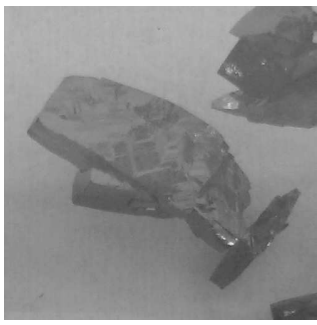

(c)

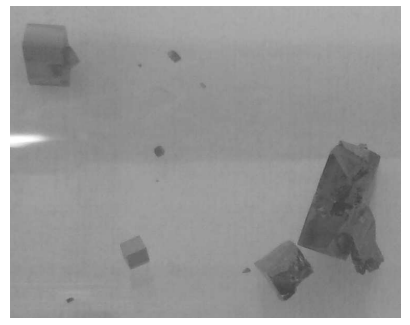

(d)

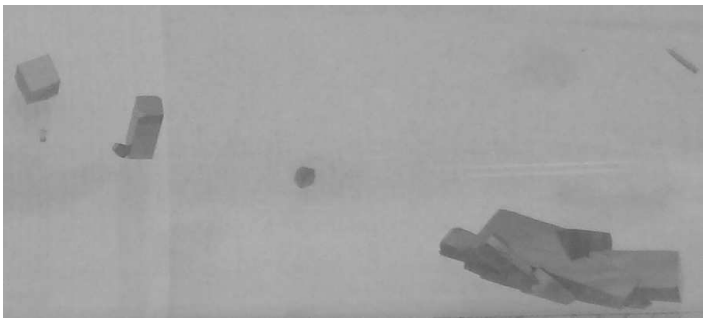

(e)

Figure 5.22. (a) Growth without pre-growth processing, with 0.02 wt- $\%$ of low $\overline{\mathbf{M}}_{\mathrm{w}}$ polyethylene mixed with the starting $\mathrm{HgI}_{2}$. (b)-(c) Twinned crystals, with the crystals acquiring the shapes most frequently found in the growth without polyethylene, grew in the beginning of the crystallization zone. (d)-(e) Smaller crystals with tetragonal prismatic shapes were found near the end of the crystallization zone. Nucleation in the area near the end was not as numerous as in the beginning of the zone.

A few observations can be made: first, halving the amount of starting $\mathrm{HgI}_{2}$ (from 80.0 $\mathrm{g}$ to $40.0 \mathrm{~g}$ ) did not exert a strong influence on $\mathrm{HgI}_{2}$ crystallization or nucleation. The same observation was also made in the growth runs without any additive (Section 5.2). Sec- 


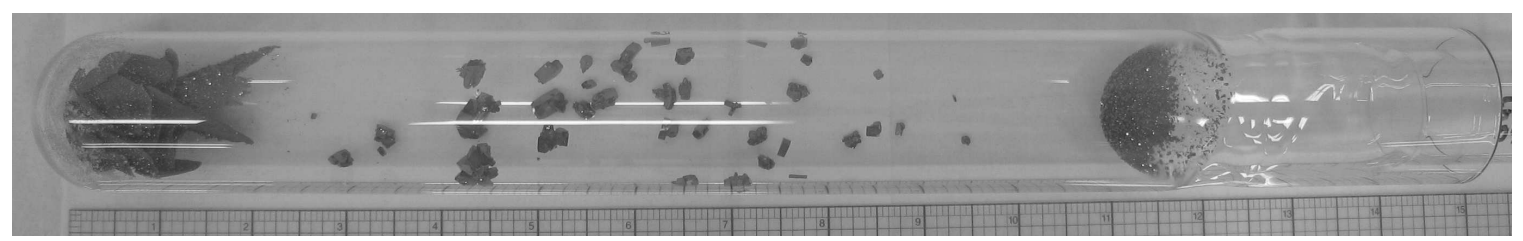

(a)

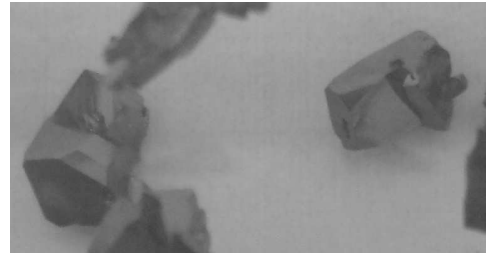

(b)

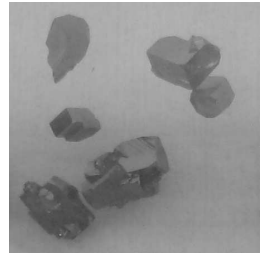

(c)

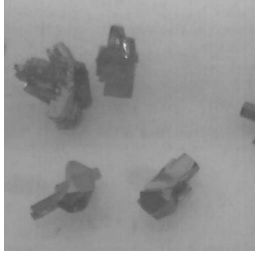

(d)

Figure 5.23. (a) Growth without pre-growth processing, with 0.5 wt- $\%$ of low $\bar{M}_{w}$ polyethylene mixed with the starting $\mathrm{HgI}_{2}$. (b)-(d) More crystals with tetragonal prismatic shapes grew, and twinning still occurred.

ond, the amount of polyethylene in the ampoule did affect crystal size, crystal shape, and frequency of nucleation. Presumably, the more polyethylene powder was present in the ampoule, the more volatiles were produced to a degree that would hinder $\mathrm{HgI}_{2}$ transport from the source to the crystallizatio sites, hence impeding nucleation and crystallization. Third, volatiles were also produced at temperatures lower than the ones used for the pre-growth processing stage. Though not numerous, droplets of organic materials were still found at the plugs, as evidenced by Fig. 5.24(c). As mentioned in the preceeding paragraphs, these growth runs were done with the source materials subjected to $100^{\circ} \mathrm{C}$, and yet the highest temperature employed during the pre-growth polyethylene processing was $230^{\circ} \mathrm{C}$ (Section 4.5.1). Whether the droplets observed in Fig. 5.24(c) were of the same types produced at higher temperatures was not known, however, these were revealed by further analysis using TD-GC/MS, as described in Section 5.7.1. 


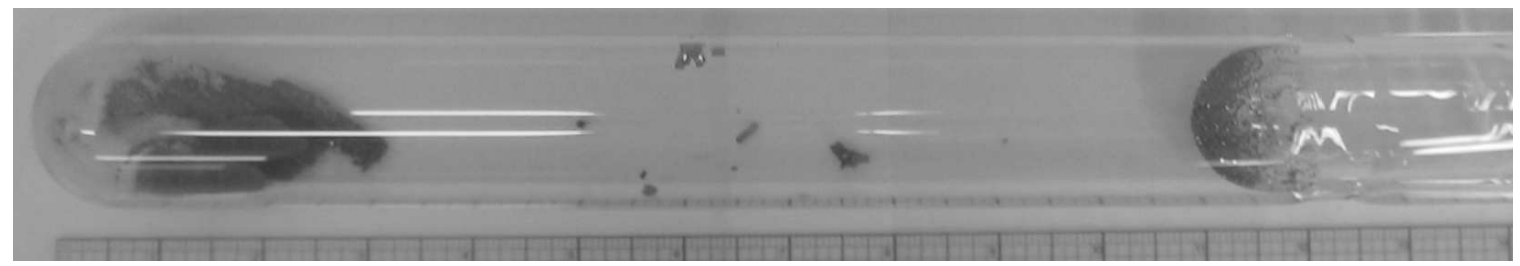

(a)

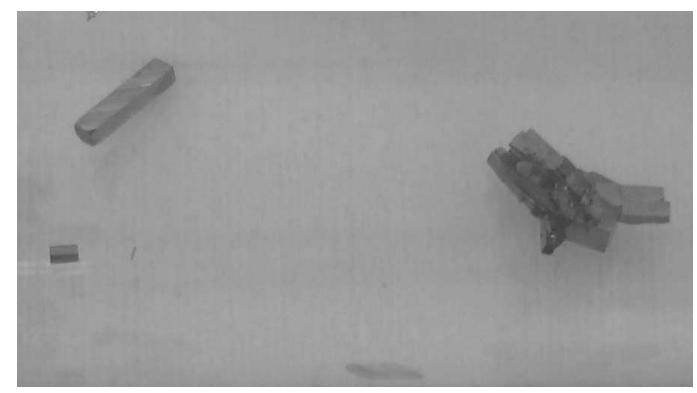

(b)

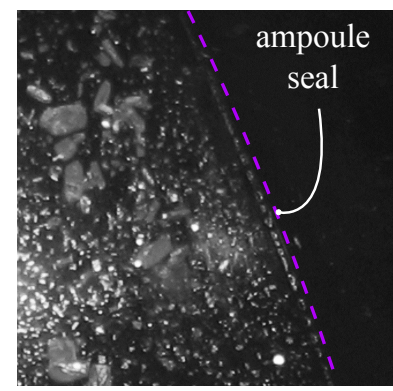

(c)

Figure 5.24. (a) Growth without pre-growth processing, with 5 wt- $\%$ of low $\overline{\mathbf{M}}_{\mathrm{w}}$ polyethylene mixed with the starting $\mathrm{HgI}_{2}$. (b) Less frequent nucleation occurred in this growth, with the crystals acquiring the tetragonal prismatic crystal shapes. Although twinning still occurred, single crystals were also found. A yellow $\mathrm{HgI}_{2}$ platelet, not found in the other ampoules, was also found. (c) Condensed organic materials at the end of the cold end of the ampoule in the vicinity of the seal (identified by a purple dotted line). 


\subsection{Growth With n-Alkanes}

Alkane homologues have been used to predict the properties of longer chain hydrocarbons, especially polyethylene $[65,66]$. Using this reasoning as a precedent, $\mathrm{HgI}_{2}$ growth runs utilizing n-alkanes were carried out. The $\mathrm{HgI}_{2}$ growth results with three different types of n-alkanes are described in this section. Section 5.5.1 details the results of growth with n-eicosane $\left(\mathrm{C}_{20} \mathrm{H}_{42}\right)$, while Sections 5.5.2 and 5.5.3 describe the results of growths with n-tetracosane $\left(\mathrm{C}_{24} \mathrm{H}_{50}\right)$ and n-hexatriacontane $\left(\mathrm{C}_{36} \mathrm{H}_{74}\right)$, respectively.

Following the observation that, in $\mathrm{HgI}_{2}$ growth ampoules with low $\overline{\mathrm{M}}_{\mathrm{w}}$ polyethylene, organic material was found condensed in the crystallization zone and on the plug, one of the hypotheses was that polyethylene degraded into shorter chain molecules, like alkanes, that subsequently volatilized and might have deposited on the condensed $\mathrm{HgI}_{2}$ on the plug. Therefore, a pre-growth polyethylene processing stage was not completed prior to the growth runs with n-alkanes.

\subsubsection{Growth with n- $\mathrm{C}_{20} \mathrm{H}_{42}$}

After a growth period of five days, almost all of the starting $\mathrm{HgI}_{2}$ crystallized, either in the crystallization zone or on the plug. The starting $n-\mathrm{C}_{20} \mathrm{H}_{42}$ was found neither in the starting end of the ampoule (Fig. 5.25(b)) nor in the considerable length of the crystallization zone (Fig. 5.25(c)). A film of condensed organic material (presumably of volatilized $n-\mathrm{C}_{20} \mathrm{H}_{42}$ ) was found near the end of the crystallization zone around the plug.

The crystal grown with $n-\mathrm{C}_{20} \mathrm{H}_{42}$ were mostly twinned, with cyclic twinning occurred frequently. A twinned platelet, with $\{110\}$ as the most prominent (hence, the largest crystal faces), can be seen in Fig. 5.26(a). Also in Fig. 5.26(a) was a smaller single crystal, with the $\{h 0 l\}$ observed. Another single crystal (Fig. 5.26(b)) had a tetragonal prismatic shape, bounded by $\{110\},(001)$, and $\{h 0 l\}$ faces. A twinned crystal in Fig. 5.27(a) also showed that $\{110\}$ were the most prominent crystal faces. The direction of growth of each twin appeared to be in the [001] direction, with the (001) face at the advancing end smaller than the (001) face at the base of the twin. In some cases (Fig. 5.27(b)), the (001) face at the 


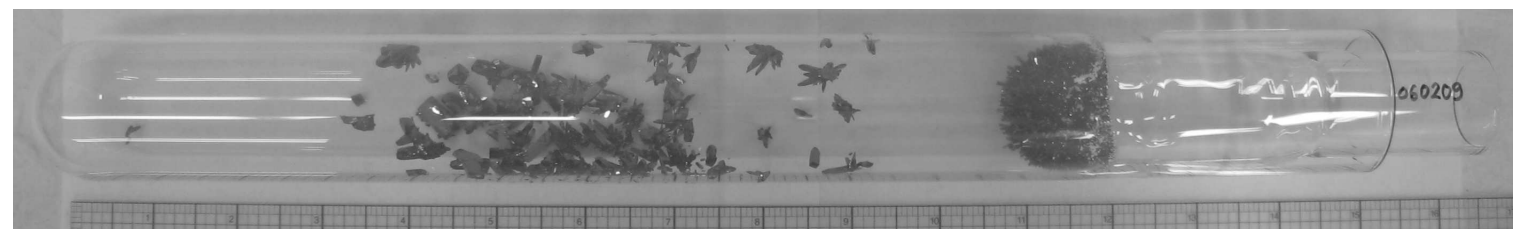

(a)

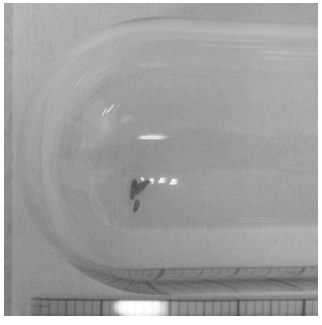

(b)

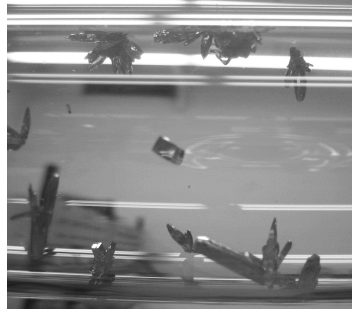

(c)

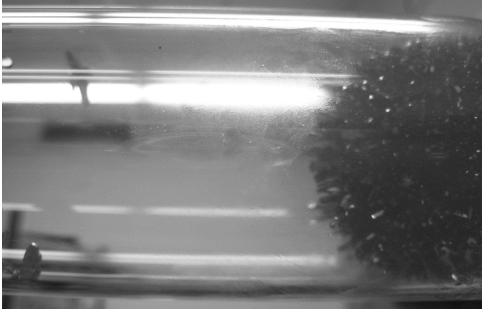

(d)

Figure 5.25. (a) $\mathrm{HgI}_{2}$ crystal growth with $n-\mathrm{C}_{20} \mathrm{H}_{42}$. (b) After a growth period of five days, most of the $\mathrm{HgI}_{2}$ crystallized in the crystallization zone and on the plug. No $\mathrm{n}-\mathrm{C}_{20} \mathrm{H}_{42}$ powder, melted or otherwise, was found in the starting end of the ampoule. (c) No organic condensation was found in a larger length of the crystallization zone. (d) Condensation of organic material was found at the end of the crystallization zone.

advancing end was very small or disappeared completely, indicating that the growth rate for (001) was faster than that for $\{110\}$. Overall, crystals growth with $n-\mathrm{C}_{20} \mathrm{H}_{42}$ had similar crystal shapes to those grown with no additive (see Section 5.2).

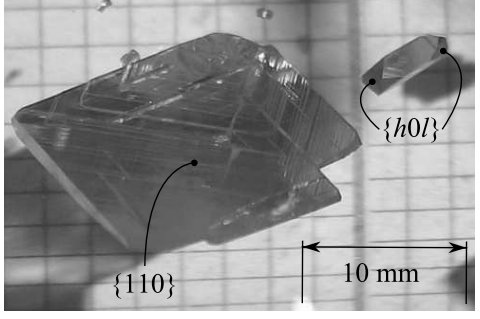

(a)

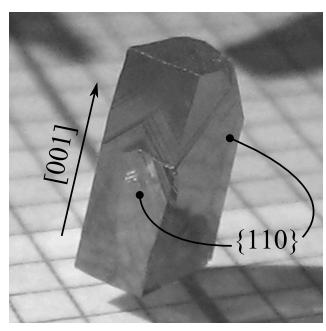

(b)

Figure 5.26. (a) A twinned platelet (left) with $\{110\}$ as the more prominent faces, which were identifiable due to the square-shaped layers. A smaller crystal (right) showing the $\{h 0 l\}$ corner faces. (b) A single tetragonal prismatic crystal.

\subsubsection{Growth with $\mathbf{n}-\mathrm{C}_{24} \mathrm{H}_{50}$}

After a growth period of five days, twinned crystals grew in the crystallization zone, in both top and bottom halves of the ampoule (Fig. 5.28(a)). Smaller twinned crystals grew 


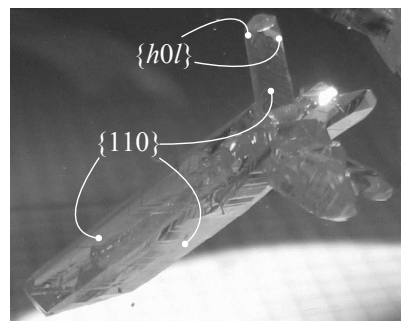

(a)

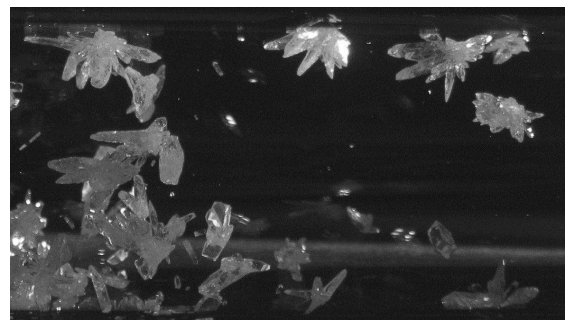

(b)

Figure 5.27. (a) Each twin in this twinned crystal had tetragonal prismatic shapes, with the advancing (001) face smaller than the (001) face at the base of the twin. (b) In some cases, the (001) face at the advancing end was either very small or disappeared completely.

in the bottom half of the ampoule, where they grew either on or surrounded by, presumably, melted $n-\mathrm{C}_{24} \mathrm{H}_{50}$. The starting $\mathrm{n}-\mathrm{C}_{24} \mathrm{H}_{50}$ was transported from the starting end of the ampoule, where it either melted and flowed into the crystallization zone, or volatilized and then condensed in the crystallization zone and around the plug area (Fig. 5.29(a)).

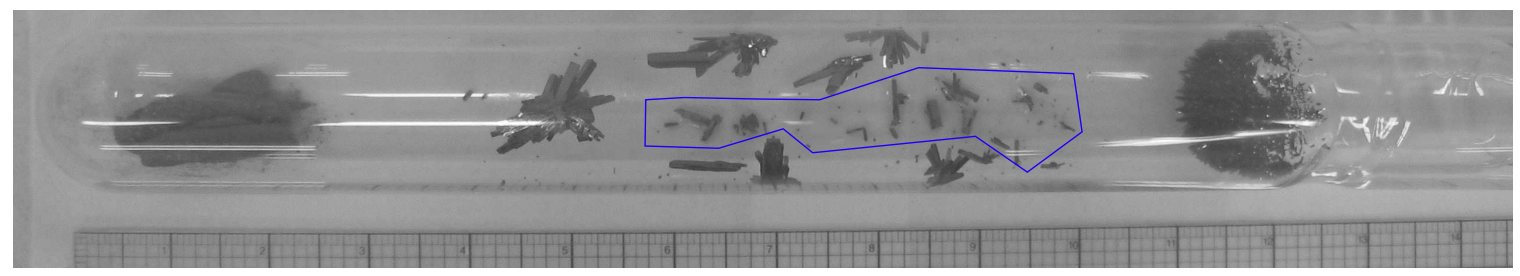

(a)

Figure 5.28. $\mathrm{HgI}_{2}$ crystal growth with $\mathrm{n}-\mathrm{C}_{24} \mathrm{H}_{50}$. Crystals enclosed in blue line were crystals growing either on or surrounded by melted $n-\mathrm{C}_{24} \mathrm{H}_{50}$ in the bottom half of the ampoule.

Crystals that grew in the bottom half of the ampoule, on or surrounded by melted n$\mathrm{C}_{24} \mathrm{H}_{50}$, were smaller in size compared to the crystals that grew away from the melted n- $\mathrm{C}_{24} \mathrm{H}_{50}$ and/or in the top half of the ampoule (Fig. 5.30(a)). Most crystals larger than 3 mm were twinned, with each twin shaped as platelet, with $\{110\}$ as the most prominent faces. Details on the surface of a crystal growing in the bottom half of the ampoule showed rhombohedral-shaped growth layers (Fig. 5.30(b)).

Fig. 5.31(a) shows a twinned crystal, with what it seemed to be a butterfly-type of twinning, growing in the top half of the ampoule (hence, it was not affected by the melted n$\mathrm{C}_{24} \mathrm{H}_{50}$ ). Each twin grew in the shape of tetragonal prisms, with one twin growing against the ampoule wall grew to about $12 \mathrm{~mm}$ in length (Fig. 5.31(a) inset). Observations on 


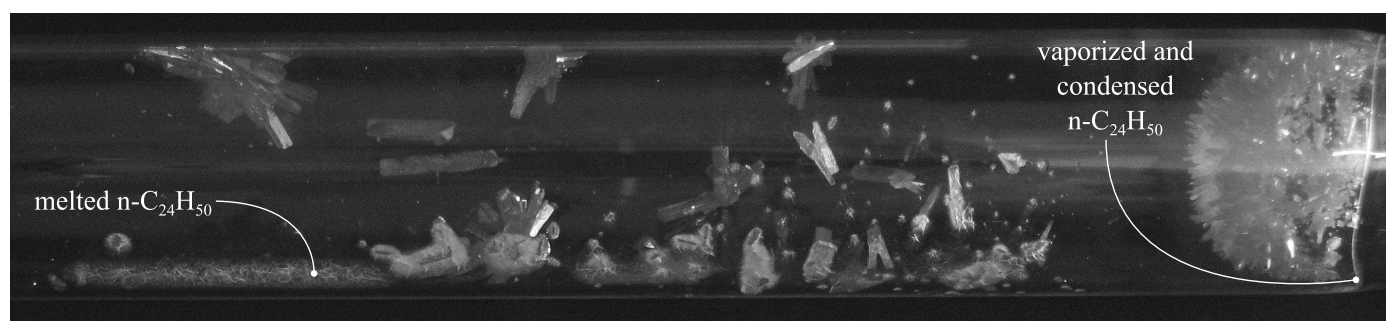

(a)

Figure 5.29. After a growth period of five days, condensing organic material (presumably $n-\mathrm{C}_{24} \mathrm{H}_{50}$ ) was found in the crystallization zone with none left in the starting end of the ampoule. Droplets of organic material were also found on the plug and around the ampoule seal.

the growth layers of this twin (Fig. 5.31(b)) revealed that the layers formed $45^{\circ}$ angle with the (001) plane for the edges growing toward the twin boundary and away from the ampoule wall. The edges growing away from the twin boundary and towards the ampoule wall formed approximately $60^{\circ}$ angle with the (001) plane. The $63.65^{\circ}$ angle indicated in Fig. 5.31(b) is the angle form between the (001) plane and rows of coplanar mercury ions in the $\{110\}$ plane (more explanation will be given in Section 5.9.5).

\subsubsection{Growth with n- $\mathrm{C}_{36} \mathrm{H}_{74}$}

After a growth period of five days, most, if not all, of the starting $\mathrm{n}-\mathrm{C}_{36} \mathrm{H}_{74}$ remained in the starting end of the ampoule, melted surrounding the starting $\mathrm{HgI}_{2}$. No visible droplets

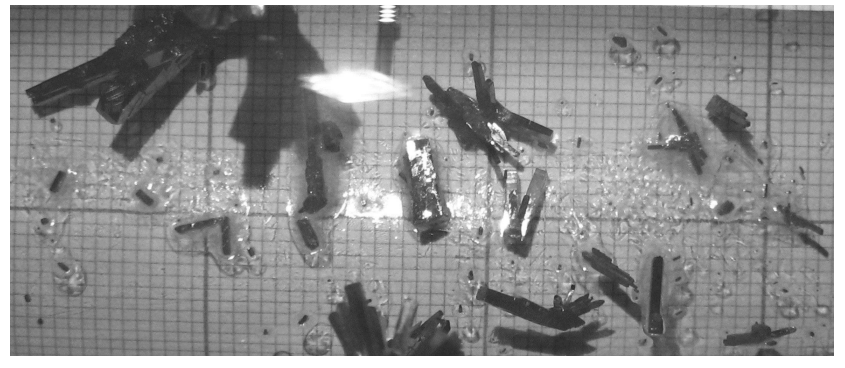

(a)

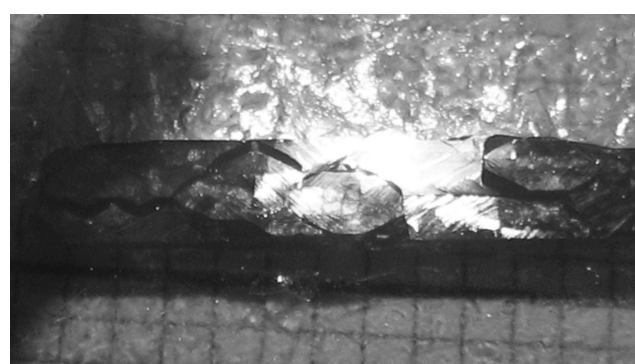

(b)

Figure 5.30. (a) Crystals growing in the bottom half of the ampoule, on or surrounded by melted $n-\mathrm{C}_{24} \mathrm{H}_{50}$, were smaller than crystals growing in the top half of the ampoule (such as crystal on the top right). (b) Details of the surface of a crystal growing in the bottom half of the ampoule. Rhombohedral-shaped layers were observed. 


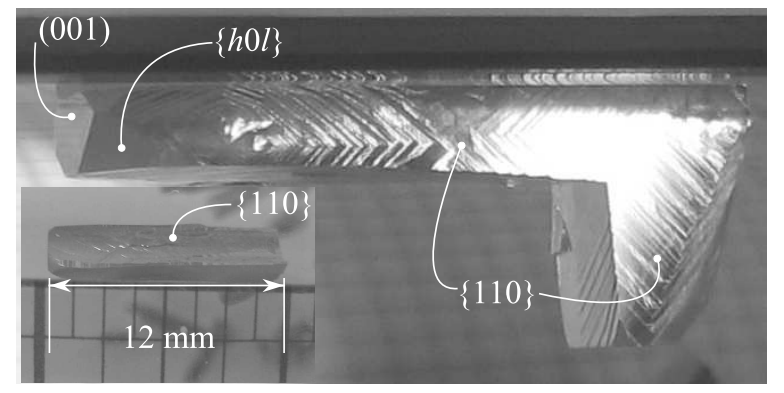

(a)

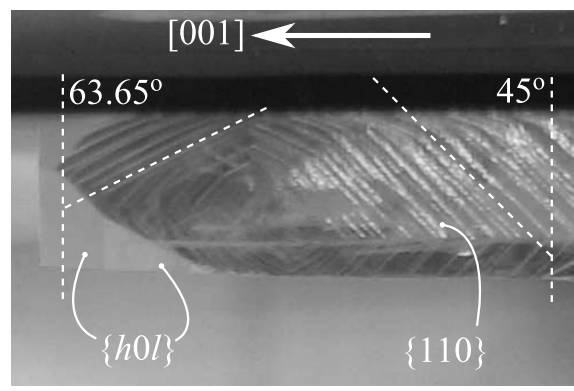

(b)

Figure 5.31. (a) A twinned crystal, growing in the upper half of the ampoule (i.e., it would have not been affected by the melted $n-\mathrm{C}_{24} \mathrm{H}_{50}$ in the crystallization zone), with one of the twin sized $\sim 12 \mathrm{~mm}$. (b) Layers on the twinned crystal, seen in (d), with the layers indicative of the $\{110\}$ faces that formed either a $63.65^{\circ}$ or $45^{\circ}$ angle with the (001) planes.

of organic material could be found either in the crystallization zone or on the plug.

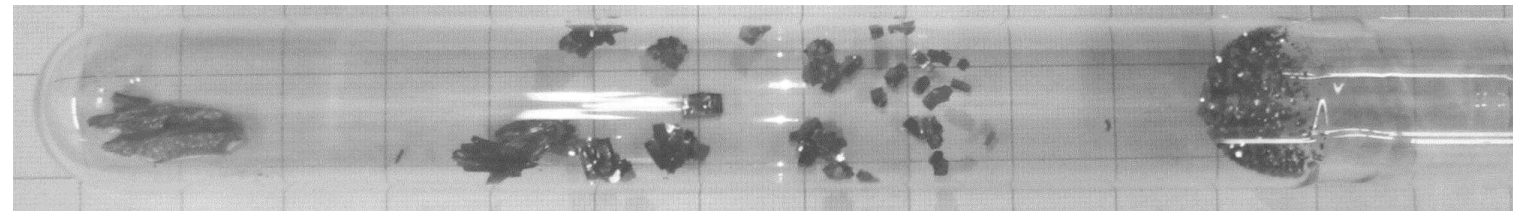

(a)

Figure 5.32. $\mathrm{HgI}_{2}$ crystal growth with $\mathrm{n}-\mathrm{C}_{36} \mathrm{H}_{74}$.

Most of the crystals were twinned, and two representatives of such crystals shown in Figs. 5.33(b) and 5.33(c). A penetration twin and a twin boundary (which might indicate the existence of a butterfly twin) were seen in a twin crystal in Fig. 5.33(b). Another twin crystal in Fig. 5.33(c) showed one of the twins growing in the direction of [001], as indicated by the cleavage (001) planes.

\subsubsection{Summary of growth with alkanes}

As mentioned in Section 5.4, finding the types of organic volatiles present during $\mathrm{HgI}_{2}$ growth with polyethylene, specifically with the low $\overline{\mathrm{M}}_{\mathrm{w}}$ polyethylene, is currently more pertinent than determining the origins of these volatiles. Nevertheless, one can suppose because normal alkanes are used as homologues of polyethylene, the presence of alkanes is a logical guess. As previously mentioned in Section 5.3.4, alkanes can be produced during 


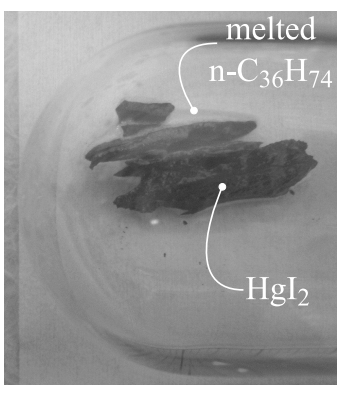

(a)

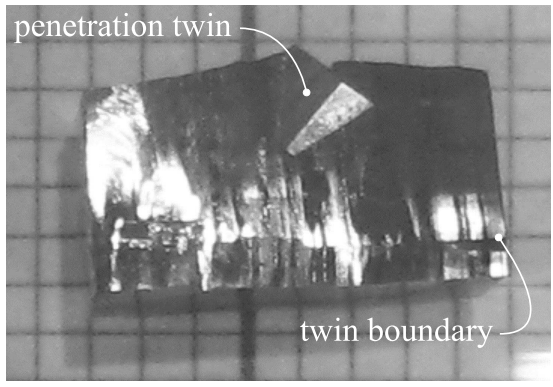

(b)

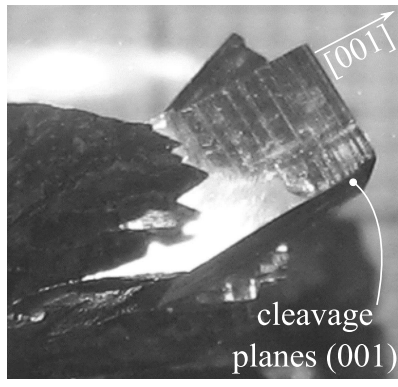

(c)

Figure 5.33. (a) After a growth period of five days, melted $n-\mathrm{C}_{36} \mathrm{H}_{74}$ was found in the starting end of the ampoule, where most of the starting $\mathrm{HgI}_{2}$ also remained. (b) A twinned crystal, with two types of twinning: butterfly or polysynthetic (with the twin boundary indicated) and penetration twins. The overall crystal shape was tetragonal prismatic. (c) Another twinned crystal, where the layers observed on one of the twins signified that the growth direction was [001].

pyrolisis of polyethylene but at temperatures above $500^{\circ} \mathrm{C}$, i.e., higher than temperatures employed during these growth procedures.

A more likely explanation for the probable presence of alkanes is that they may be present as impurities in the starting polyethylene. One method to produce low $\overline{\mathbf{M}}_{\mathrm{w}}$ polyethylene is by direct polymerization of ethylene, with either a high or low pressure polymerization (which produces low or high density polyethylene, respectively), while maintaining certain conditions that control the molecular weight [67], i.e., chain length. Therefore, it is likely that during this synthesis of low $\overline{\mathrm{M}}_{\mathrm{w}}$ polyethylene, alkanes or shorter chains of polyethylene are produced inadvertantly as impurities.

Alkanes used during the growth were chosen prior to the analysis of the low molecular weight polyethylene by thermal desorption (TD)-GC/MS, which later reveals that, indeed, various alkanes were among the chemicals desorped from heating low molecular weight polyethylene at $100^{\circ} \mathrm{C}$ and $150^{\circ} \mathrm{C}$ (Sections 4.8.1 and 5.7.1). These alkanes were chosen primarily to observe the effect of chain length or molecular weight on $\mathrm{HgI}_{2}$ growth in the horizontal furnace by the Faile method. The premise that the $\mathrm{HgI}_{2}$ crystal morphology obtained by the Faile method was contingent on mass transport was again the reason behind choosing these alkanes. 


\subsection{Growth with Ketones}

$\mathrm{HgI}_{2}$ growth runs with ketones were commenced after the TD-GC/MS results were obtained (Section 5.7.1). Section 5.6.1 describes the results of $\mathrm{HgI}_{2}$ growth with 3-hexadecanone, while Section 5.6.2 describes the results of $\mathrm{HgI}_{2}$ growth with 14-heptacosanone.

\subsubsection{Growth with 3-hexadecanone}

After a growth period of five days, single and twin crystals were obtained in $\mathrm{HgI}_{2}$ growth with 0.1 wt- $\%$ of 3 -hexadecanone (Fig. 5.34). The single crystals had platelet shapes, with (001) as the most prominent faces (Fig. 5.34(b)). The growth direction of these platelets (i.e., direction perpendicular to ampoule wall) was the [001] direction (Figs. 5.34(b) - 5.34(c)). Very small $\{h 0 l\}$ faces could be observed, however, not large enough to alter the shape of the platelets (Fig. 5.34(b)). Twin crystals were also obtained, with visible (001), $\{110\}$, and $\{h 0 l\}$ faces. The position of (001) indicated that the growth direction of each twin was also the [001] direction (Fig.5.34(d)). No condensation of organic material was found in the crystallization zone. 


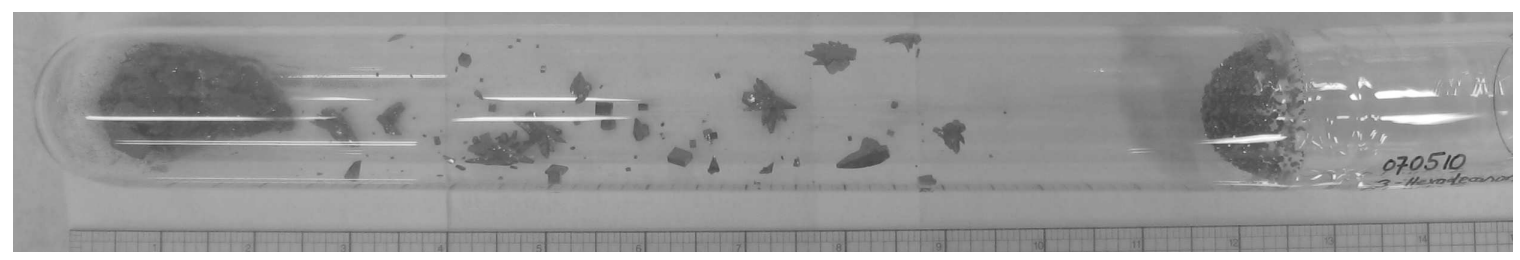

(a)

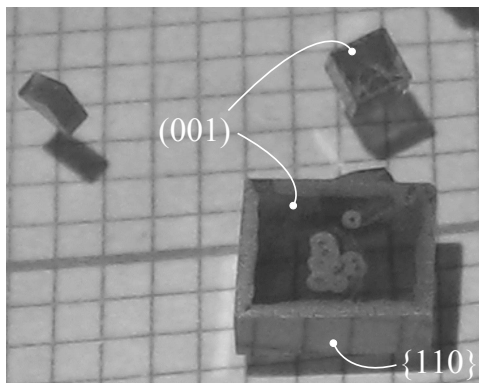

(b)

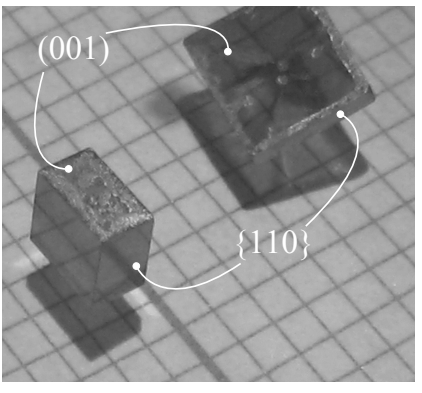

(c)

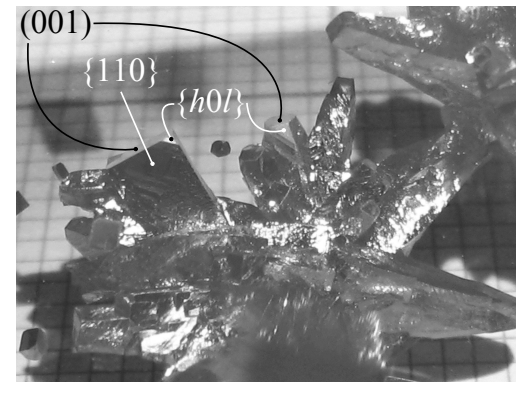

(d)

Figure 5.34. (a) $\mathrm{HgI}_{2}$ growth with $0.1 \mathrm{wt}-\%$ of 3 -hexadecanone. (b) Single crystals mostly grew as platelets, with (001) as the most prominent face. The growth direction for both platelets was [001]. (c) Either platelet (top right) or tetragonal prismatic-shaped (bottom left) crystals grew, with [001] as the growth direction, perpendicular to the ampoule wall. (d) Twin crystals also grew and were larger than the single crystals. Tetragonal prismatic twins were observed, with square-shaped growth layers observed on $\{110\}$. 


\subsubsection{Growth with 14-heptacosanone}

Single and twinned $\mathrm{HgI}_{2}$ crystals were obtained in growth with $0.1 \mathrm{wt}-\%$ of 14-heptacosanone after a growth period of five days (Fig. 5.35(a)). As in the growth run with 3-hexadecanone, the twin crysals were larger compared to the single crystals. The single crystals grew in tetragonal prismatic shapes, with growth direction [001] perpendicular to the ampoule wall (Figs. 5.35(b) - 5.35(c)). A detailed look at one of the twin crystals shows that the growth direction [001] was parallel to the ampoule wall and the $\{110\}$ face featured rhombohedral-shaped growth layers (Fig. 5.35(e)). The angle between the edge of the growth layers and the (001) face could be fitted to a $63.65^{\circ}$ angle. The crystal shapes were indeed similar to the shapes of crystals grown with the low $\overline{\mathrm{M}}_{\mathrm{w}}$ polyethylene (for example, Fig. 5.12). Condensed organic material, presumably volatilized 14-heptacosanone, was found in throughout the length of the crystallization zone.

As mentioned in Table 4.6, only $0.1 \mathrm{wt}-\%$ of 14 -heptacosanone was used in the growth ampoule. When 1 wt- $\%$ of 14-heptacosanone was used, much smaller crystals, mostly twinned, were obtained after a growth period of five days (Fig. 5.36(a), a more detailed view in Fig. 5.36(b)). This growth result is similar to $\mathrm{HgI}_{2}$ growth without addition, but with approximately 4.2 Torr $\mathrm{N}_{2}$ back-fill pressure after ampoule evacuation (Section 5.2.2). One of the single crystals (Fig. 5.36(c)) featured $\{110\}$ as the most prominent faces. The [001] direction, again, was the faster growth direction, and it was perpendicular to the ampoule wall. 


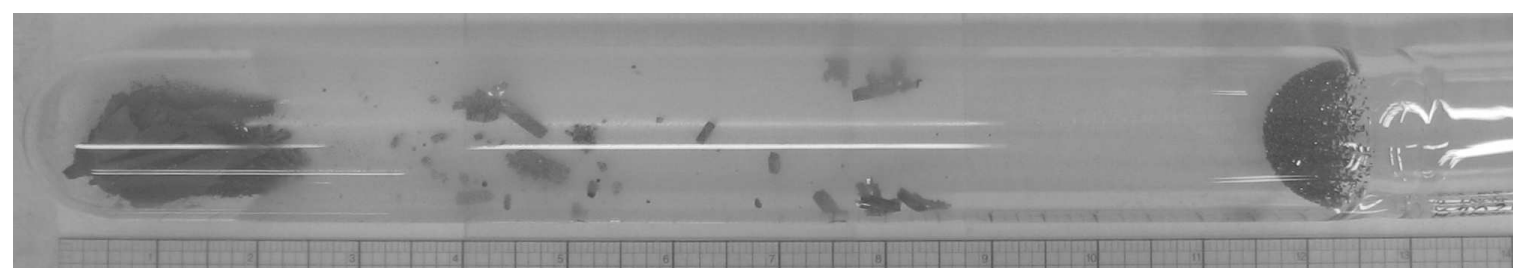

(a)

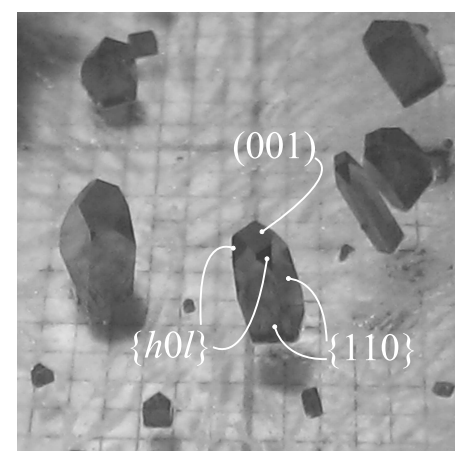

(b)

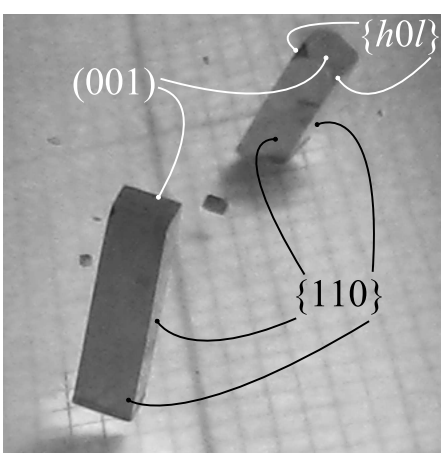

(c)

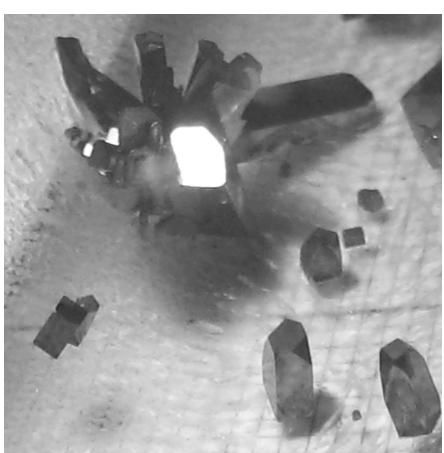

(d)

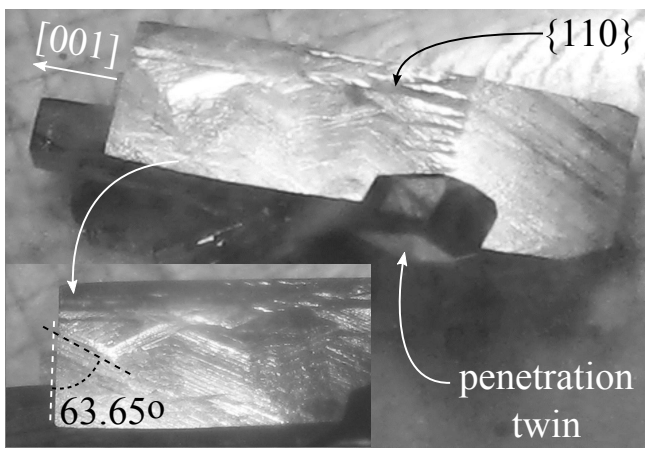

(e)

Figure 5.35. (a) $\mathrm{HgI}_{2}$ growth with $0.1 \mathrm{wt}-\%$ 14-heptacosanone. (b) Tetragonal prismatic crystals, with (001), $\{110\}$, and $\{h 0 l\}$ faces observed. The growth direction was [001], which was perpendicular to the wall. (c) Two tetragonal prismatic crystals, with much smaller $\{h 0 l\}$ faces than the crystals in (a). (d) Twin crystals still grew, with sizes larger than the single crystals. (e) A twin crystal with a penetration twin. The [001] axis appears to be parallel to the ampoule wall. Details of the $\{110\}$ top face (inset) show that the angle between the edge of growth layers and the (001) face could be fit by a $63.65^{\circ}$ angle. 


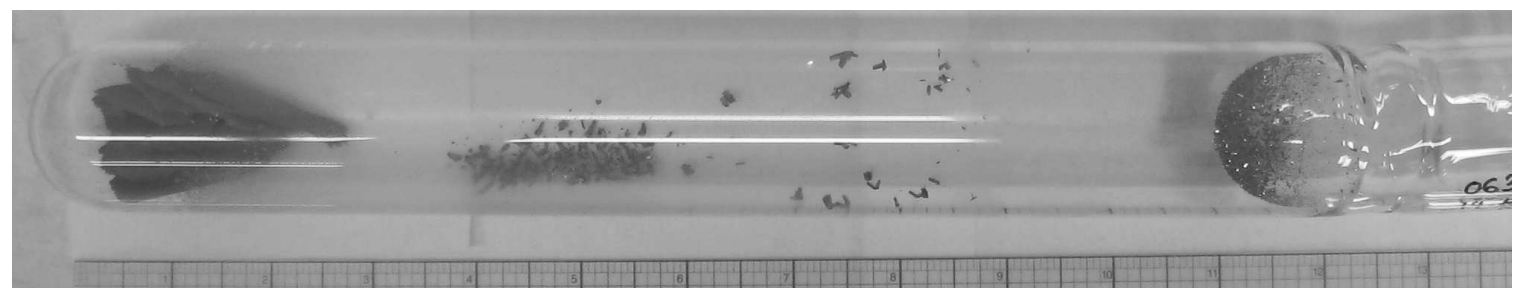

(a)

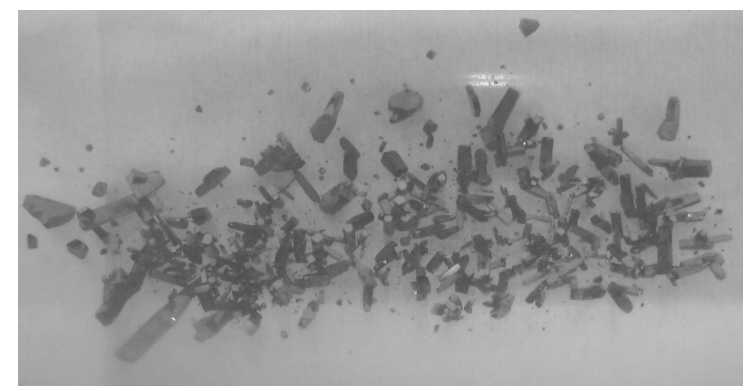

(b)

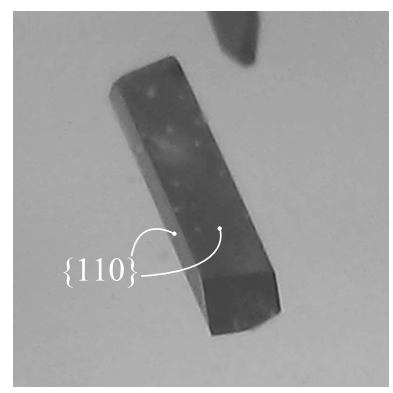

(c)

Figure 5.36. (a) $\mathrm{HgI}_{2}$ growth with $1 \mathrm{wt}-\%$ of 14-heptacosanone. (b) Much smaller and thinner crystals, mostly twinned, grew in the presence of a large amount of 14-heptacosanone. (c) One of the single crystals, with $\{110\}$ as the most prominent faces. The white dots seen were condensed organic material on the ampoule wall. 


\subsection{Characterization of Polyethylene and n-Alkanes}

The results of the TD-GC/MS studies on the low $\overline{\mathrm{M}}_{\mathrm{w}}$ polyethylene, the ultra high $\overline{\mathrm{M}}_{\mathrm{w}}$ polyethylene and n-eicosane $\left(\mathrm{C}_{20} \mathrm{H}_{42}\right)$ are described in Section 5.7.1. Examination results on the $\mathrm{HgI}_{2}$ as-grown and cleaved surfaces, as well as processed low $\overline{\mathrm{M}}_{\mathrm{w}}$ polyethylene, are shown in Section 5.7.2.

\subsubsection{TD-GC/MS study of polyetylene and n-alkanes}

Fig. 5.37 shows a gas chromatograph with the results of thermal desorption of low $\overline{\mathrm{M}}_{\mathrm{w}}$ polyethylene at $100^{\circ} \mathrm{C}$, where peaks corresponding to dodecene $\left(\mathrm{C}_{12} \mathrm{H}_{24}\right)$ isomers, methyl (even-n) alkyl ketones, and odd n-alkanes were observed (Crossed out peaks are background). At $150^{\circ} \mathrm{C}$, peaks associated to longer methyl (even-n) alkyl ketones and odd n-alkanes were observed (Fig. 5.38 ). The absence and/or the height reduction of several peaks observed in the $100^{\circ} \mathrm{C}$ thermal desorption (Fig. 5.37) imply that molecules associated with those peaks no longer present at higher temperatures, rather longer chain molecules were produced.

The TD-GC/MS test on the ultra high $\overline{\mathrm{M}}_{\mathrm{w}}$ polyethylene (Fig. 5.39) shows that peaks other than background were considerably smaller at $100^{\circ} \mathrm{C}$, and tests performed at $150^{\circ} \mathrm{C}$ (not shown), $200^{\circ} \mathrm{C}$ (not shown), and $230^{\circ} \mathrm{C}$ virtually did not show any peak associated with

the ultra high $\overline{\mathrm{M}}_{\mathrm{w}}$ polyethylene or any other organics. This result means that either ultra high $\overline{\mathrm{M}}_{\mathrm{w}}$ polyethylene did not volatilize or the kinds and amounts of molecules produced were below the detection limit. The TD-GC/MS test on n-eicosane $\left(n-\mathrm{C}_{20} \mathrm{H}_{42}\right)$ was done at $100^{\circ} \mathrm{C}$ and the chromatograph is shown in Fig. 5.40. Other than the background peaks, only one peak associated with $n-\mathrm{C}_{20} \mathrm{H}_{42}$ was observed, which means that $\mathrm{n}-\mathrm{C}_{20} \mathrm{H}_{42}$ did volatilize and yet did not undergo scission or produce shorter chain molecules. 


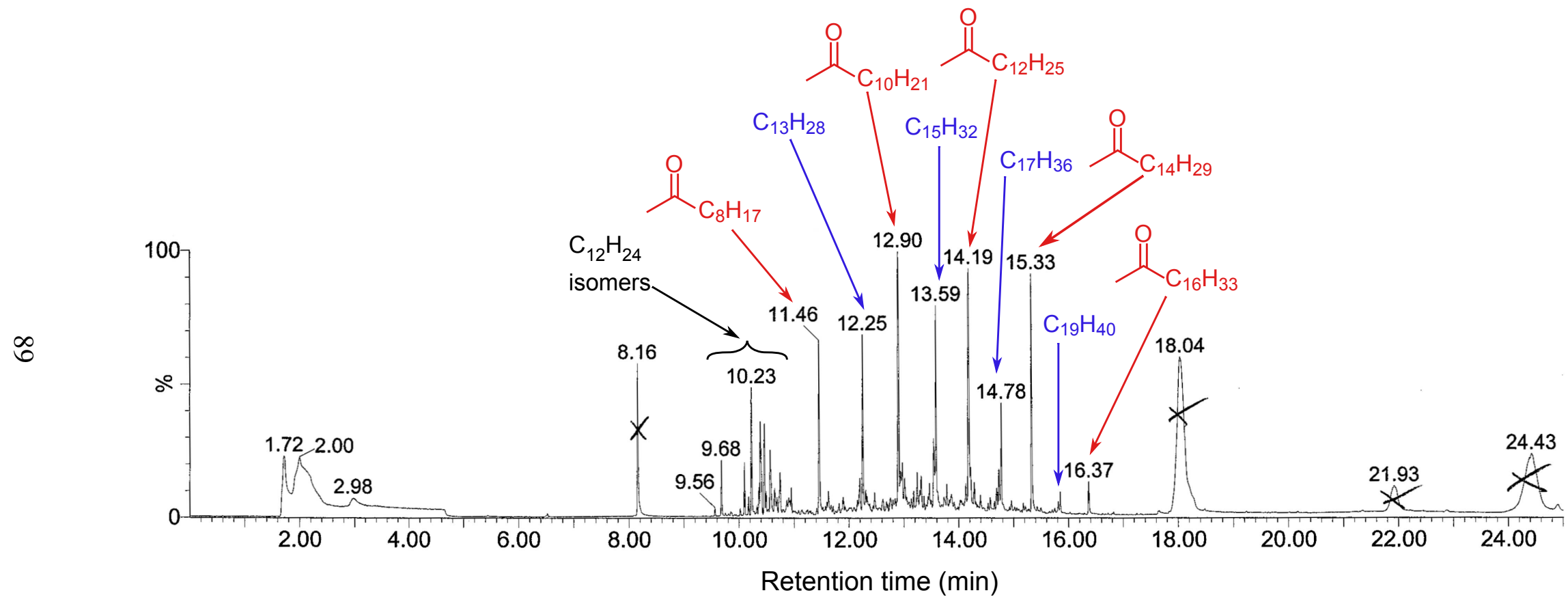

Figure 5.37. Gas chromatograph for thermal desorption of low $\overline{\mathrm{M}}_{\mathrm{w}}$ polyethylene at $100^{\circ} \mathrm{C}$. Peaks corresponding to dodecene $\left(\mathrm{C}_{12} \mathrm{H}_{24}\right)$ isomers, methyl (even-n) alkyl ketones, and odd n-alkanes were observed. Crossed out peaks are background. 


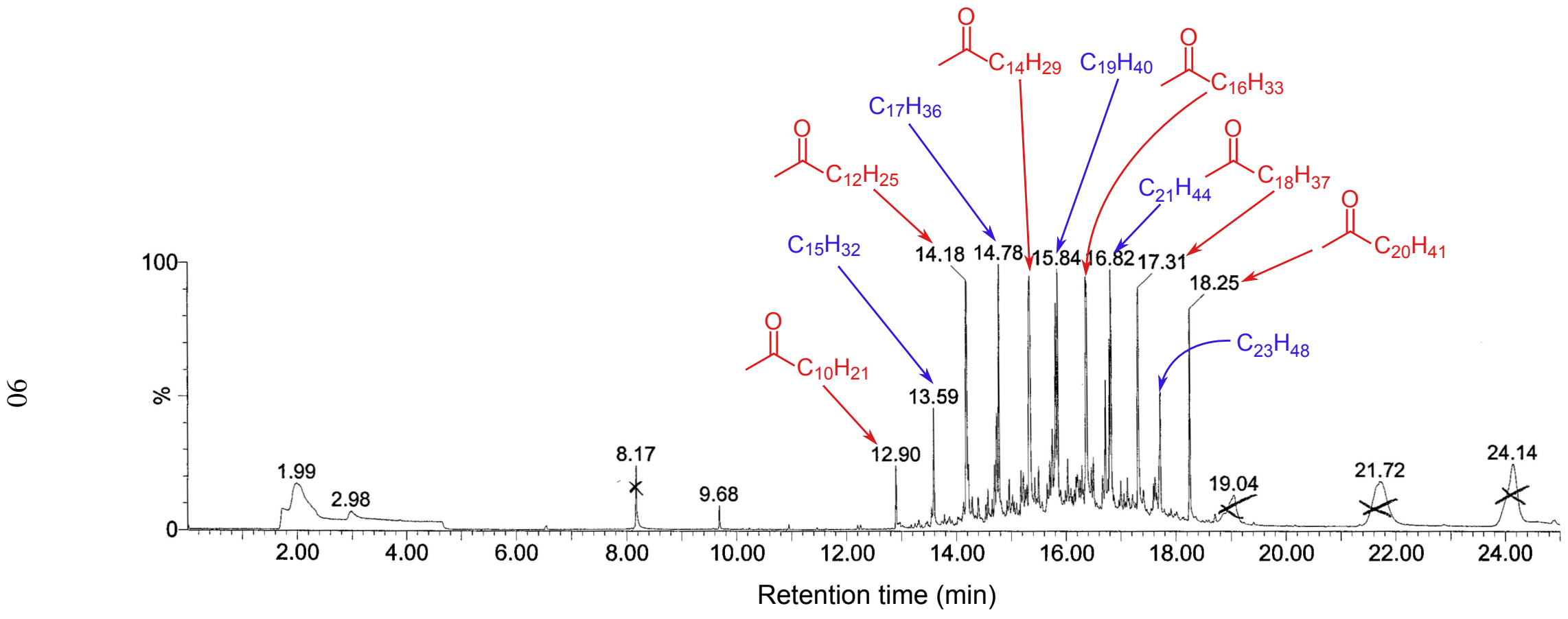

Figure 5.38. Gas chromatograph for thermal desorption of low $\overline{\mathrm{M}}_{\mathrm{w}}$ polyethylene at $150^{\circ} \mathrm{C}$. Peaks associated to longer methyl (even-n) alkyl ketones and odd n-alkanes were observed. The absence and/or the height reduction of several peaks observed in the $100^{\circ} \mathrm{C}$ thermal desorption (Fig. 5.37) imply that molecules associated with those peaks no longer present at higher temperatures, rather longer chain molecules were produced. 


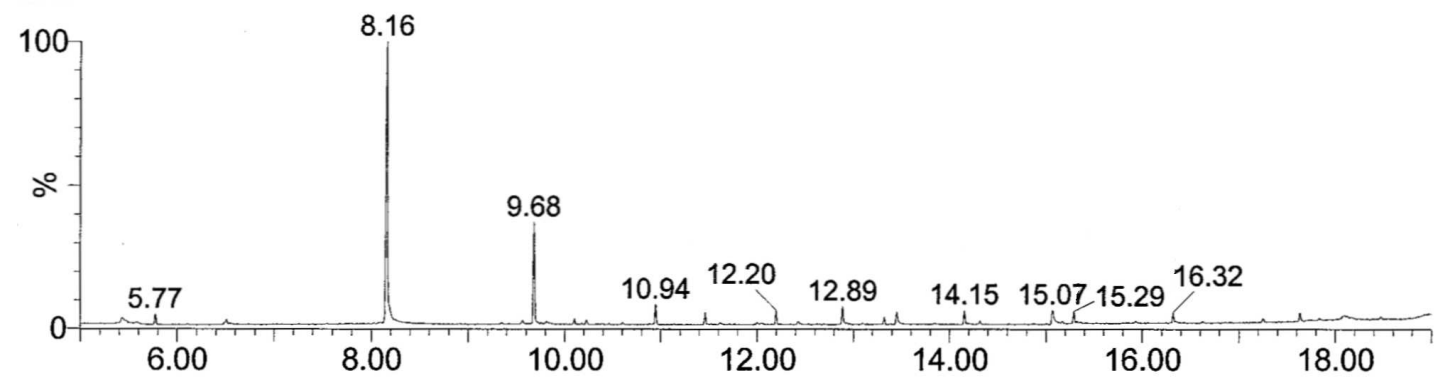

(a) at $100^{\circ} \mathrm{C}$

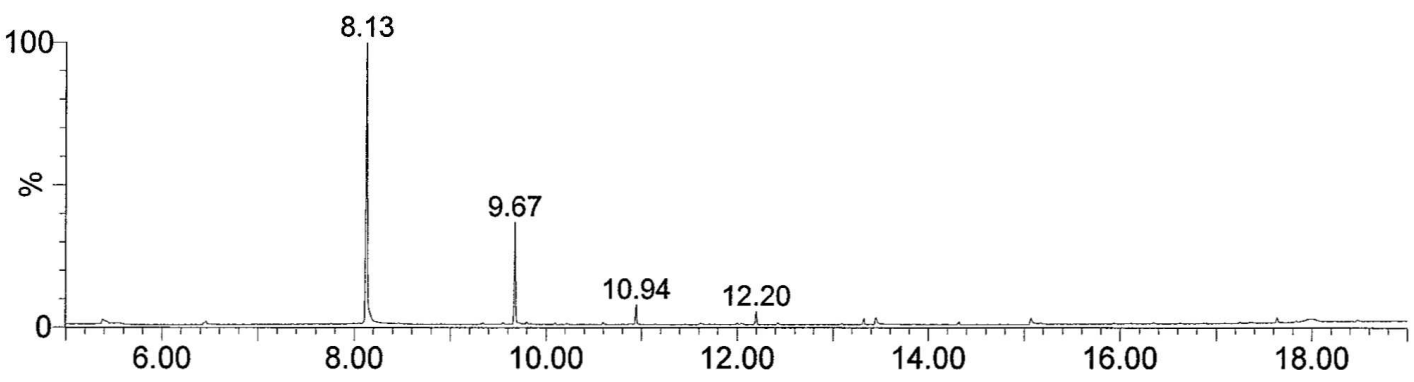

(b) at $230^{\circ} \mathrm{C}$

Figure 5.39. Gas chromatograph for thermal desorption of ultra high $\overline{\mathrm{M}}_{\mathrm{w}}$ polyethylene. (a) Peaks considerably smaller than the background were observed at $100^{\circ} \mathrm{C}$. (b) Tests at $150^{\circ} \mathrm{C}$ (not shown), $200^{\circ} \mathrm{C}$ (not shown), and $230^{\circ} \mathrm{C}$ virtually did not show any peak associated with the ultra high $\overline{\mathrm{M}}_{\mathrm{w}}$ polyethylene or any other organics. This means that either ultra high $\overline{\mathrm{M}}_{\mathrm{w}}$ polyethylene did not volatilize or the kinds and amounts of molecules produced were below the detection limit. 


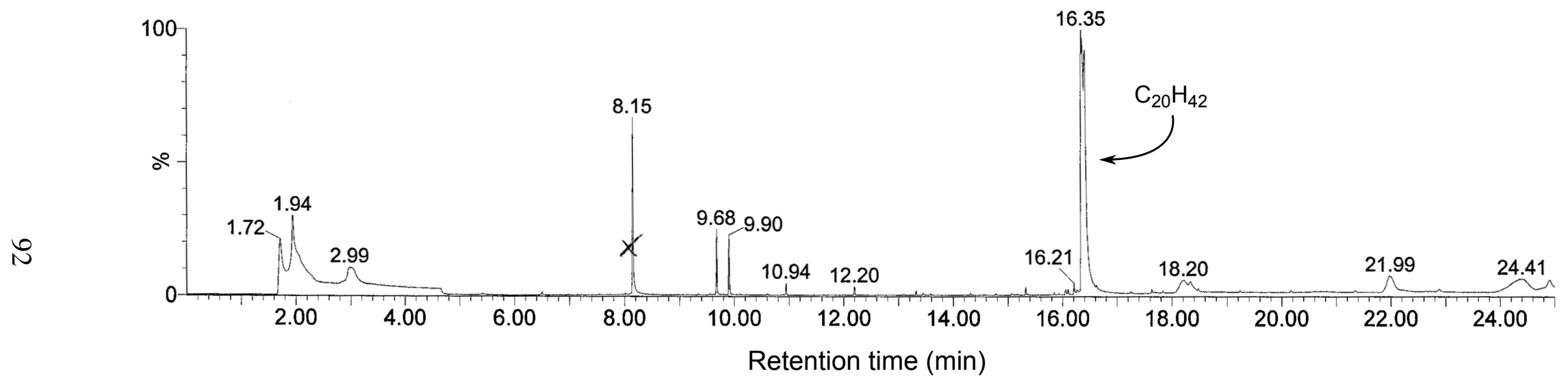

Figure 5.40. Gas chromatograph for thermal desorption of n-eicosane $\left(n-\mathrm{C}_{20} \mathrm{H}_{42}\right)$. Other than the background peaks, only one peak associated with $n-\mathrm{C}_{20} \mathrm{H}_{42}$ was observed. This means that $-\mathrm{C}_{20} \mathrm{H}_{42}$ did volatilize and yet did not undergo scission or produce shorter chain molecules. 
"Pure" polyethylene is not expected to have carbonyl groups or triple bonds, however, it is known that polyethylene does contain impurities, such as metal catalyst residues, carbonyl compounds, hydroperoxides, and double bonds [68]. Therefore, it is conceivable that these impurities were the volatiles whose peaks appeared in the gas chromatograph from the TD-GC/MS test on low $\overline{\mathrm{M}}_{\mathrm{w}}$ polyethylene. The TD-GC/MS process was done at ambient pressure but in $\mathrm{He}\left(\mathrm{O}_{2}\right.$ free $)$ atmosphere, excluding the possibility of oxidation, which means that the observed ketones, alkanes, and alkenes were impurities in the starting low $\overline{\mathrm{M}}_{\mathrm{w}}$ polyethylene.

\subsubsection{ATR-IR spectroscopy}

Background subtracted ATR-IR spectrum of processed polyethylene is seen Fig. 5.41, where bands characteristics of alkanes and ketones were identified, though the types of alkanes or ketones could not be differentiated. The processed polyethylene sample was obtained from the pre-growth polyethylene processing with low $\overline{\mathrm{M}}_{\mathrm{w}}$ polyethylene, where the polyethylene powder was mixed with starting $\mathrm{HgI}_{2}$ in an ampoule, which was subsequently sealed under vacuum and subjected to $150-230^{\circ} \mathrm{C}$, which were high enough temperatures to desorp or release impurities such as ketones, alkanes, and alkenes from the polyethylene, according to the results of TD-GC/MS (Figs. 5.37 and 5.38).

The infrared characteristics bands of ketones or alkanes could not be found on the ATRIR examination on the surface of either as-grown $\{110\}$ or cleaved (001) faces (Fig. 5.42). Because the crystal surfaces were in direct contact with the diamond ATR crystal, the absence of any infrared characteristics bands in the spectra suggests that no ketones, alkanes, or alkenes, were on the as-grown $\{110\}$ faces or that the quantities of these organics were not significant to amount any response above the background. The absence of any characteristics bands also means that no organics were incorporated into the bulk of the crystal. 


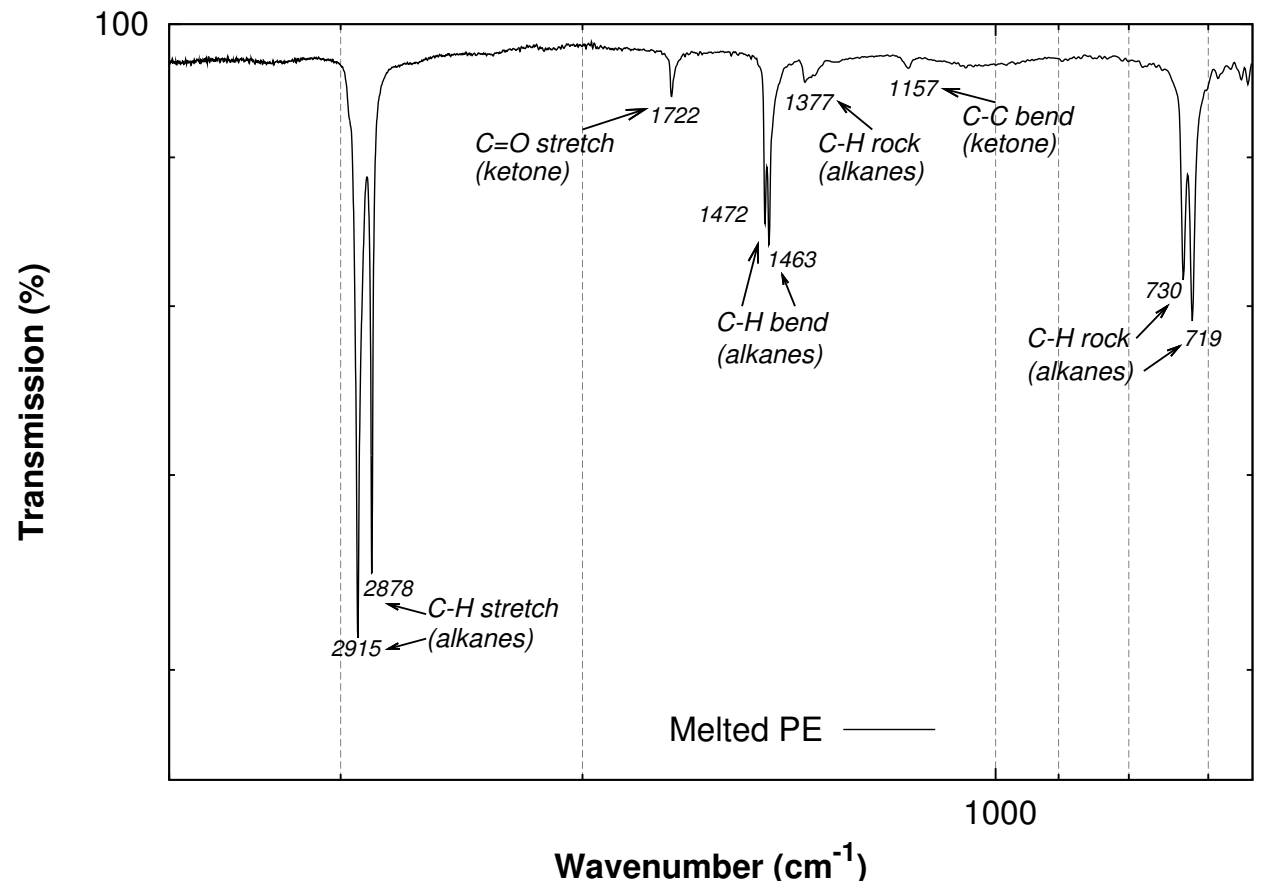

Figure 5.41. Background subtracted of ATR-IR spectrum of processed low $\bar{M}_{w}$ polyethylene.

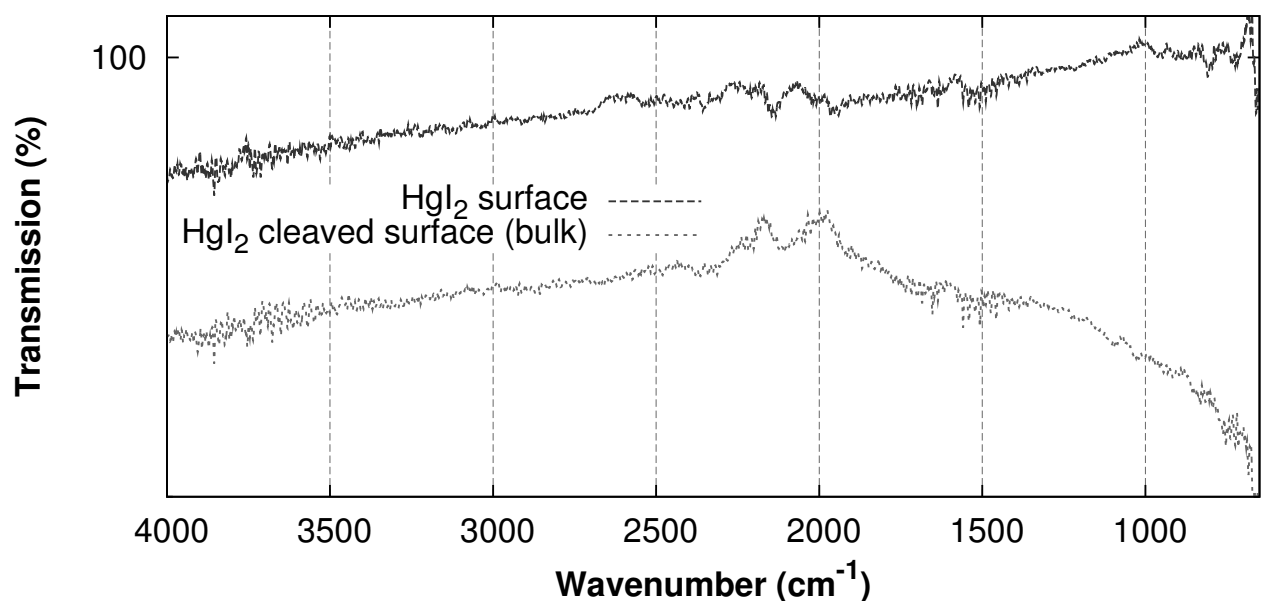

Figure 5.42. Background subtracted of ATR-IR spectra of as-grown (110) and cleaved (001) faces of $\mathrm{HgI}_{2}$ crystals. Peaks between 2500 and $2000 \mathrm{~cm}^{-1}$ are artifacts from background subtraction. 


\section{8 $\mathrm{HgI}_{2}$ Frisch Collar Detector}

For this study the pulse height spectra were collected from standard commercially available calibration sources of ${ }^{241} \mathrm{Am},{ }^{57} \mathrm{Co},{ }^{133} \mathrm{Ba},{ }^{137} \mathrm{Cs}$, and ${ }^{60} \mathrm{Co}$. A neutron activated ${ }^{198} \mathrm{Au}$

pulse height spectrum was also collected. The ${ }^{198} \mathrm{Au}$ source was activated by irradiating a $13 \mathrm{mg}$ gold foil sample in the Kansas State University TRIGA Mk II nuclear reactor core for 8 minutes at $5 \mathrm{~kW}$. The electronic settings were consistent for all measurements, and energy resolutions reported for gamma-ray full energy peaks are without any electronic corrections.

An energy resolution of $4.7 \%$ FWHM for the $59.5 \mathrm{keV}$ spectral line of ${ }^{241} \mathrm{Am}$ was obtained, shown in Fig. 5.43. Shown in Fig. 5.44 is ${ }^{57}$ Co spectrum where the energy resolution at the $122 \mathrm{keV}$ is $4.0 \% \mathrm{FWHM}$, and the $136 \mathrm{keV}$ energy line is clearly observed. An energy resolution of $4.4 \%$ FWHM was achieved for the $80 \mathrm{keV}$ spectral line of ${ }^{133} \mathrm{Ba}$ (see Fig. 5.45). The $356 \mathrm{keV}$ photopeak of ${ }^{133} \mathrm{Ba}$ exhibited an energy resolution of $2.5 \%$ FWHM (see Fig. 5.46), with the $276 \mathrm{keV}, 302 \mathrm{keV}$, and $382 \mathrm{keV}$ emission energies of ${ }^{133} \mathrm{Ba}$ are also clearly observable.

The ${ }^{198} \mathrm{Au}$ spectrum is shown in Fig. 5.47 where the $411 \mathrm{keV}$ full energy peak energy resolution is $2.6 \%$ FWHM. The $662 \mathrm{keV}$ photopeak of ${ }^{137} \mathrm{Cs}$ with $1.8 \%$ FWHM energy resolution is also shown in Fig. 5.48. The $\mathrm{Hg}$ x-ray escape peaks are observable on both spectra of ${ }^{198} \mathrm{Au}$ and ${ }^{137} \mathrm{Cs}$ (Figs. 5.47 and 5.48). Fig. 5.49 displays the energy spectrum from ${ }^{60} \mathrm{Co}$ collected with the $\mathrm{HgI}_{2}$ Frisch collar device. As shown, an energy resolution of $1.2 \% \mathrm{FWHM}$ at $1173 \mathrm{keV}$ energy line is achieved. Note that the relatively small $\mathrm{HgI}_{2}$ device was capable of detecting the high energy gamma rays emitted from ${ }^{60} \mathrm{Co}$.

The stability of the detector with time is also reported in Fig. 5.50, showing ${ }^{137} \mathrm{Cs}$ energy energy spectra over various irradiation times after initial operation. When the $\mathrm{HgI}_{2}$ Frisch collar detector had been under bias for more than 24 hours, the pulse height spectrum remained more or else the same. In other words, the detector did not show polarization beyond 24 hours of operation at high voltage.

Figs. 5.51 and 5.52 show the collected spectra with the $2.1 \times 2.1 \times 4.1 \mathrm{~mm}^{3} \mathrm{HgI}_{2}$ 


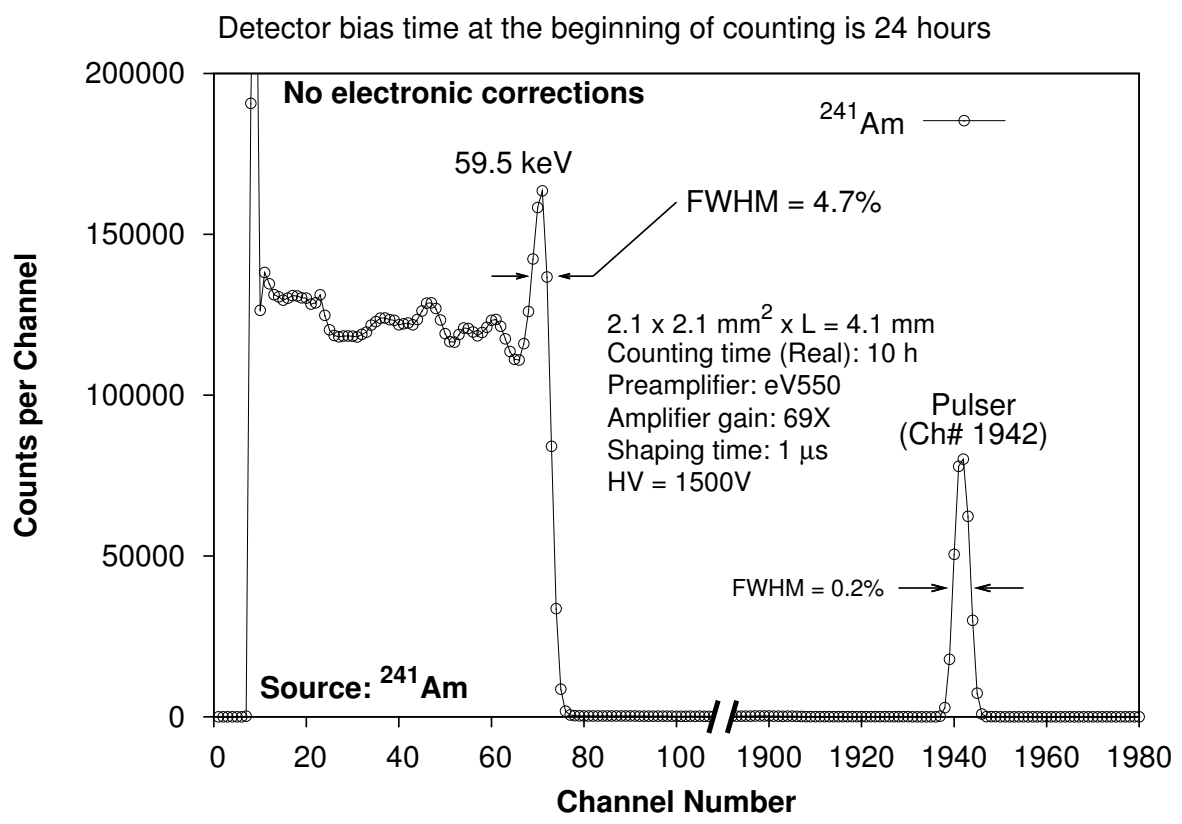

Figure 5.43. Pulse height spectrum taken with a $2.1 \times 2.1 \times 4.1 \mathrm{~mm}^{3} \mathrm{HgI}_{2}$ Frisch collar device being fully irradiated with a ${ }^{241} \mathrm{Am}$ gamma ray source positioned $3.0 \mathrm{~cm}$ away from the detector side. A $4.7 \%$ FWHM energy resolution is achieved at $59.5 \mathrm{keV}$.

Frisch collar device operated at $1500 \mathrm{~V}$ from the collimated ${ }^{137} \mathrm{Cs}$ gamma-ray source. The collected spectra show the uniformity of the device response to gamma-rays along the device length. This uniformity is further confirmed by plotting the normalized peak channel number of each spectrum versus the irradiated points(Fig. 5.53). As plotted in Fig. 5.53, the peak channel of the photo peak remained unchanged as the collimated gamma-ray source probed more than half of the device length (points 1 through 4). Applying the CCE model to the $2.1 \times 2.1 \times 4.1 \mathrm{~mm}^{3} \mathrm{HgI}_{2}$ Frisch collar device at $1500 \mathrm{~V}$, results in the estimated values of electrons and holes mobility-lifetime products $\mu_{e, h} \tau_{e, h}$ of $0.0008 \mathrm{~cm}^{2} \mathrm{~V}^{-1}$ and $0.00003 \mathrm{~cm}^{2} \mathrm{~V}^{-1}$ for the grown $\mathrm{HgI}_{2}$ crystal. The $\mu_{e, h} \tau_{e, h}$ values for the $\mathrm{HgI}_{2}$ were evaluated by trial and error to fit the best CCE profile through the peak channel of the photo peak as presented in Fig. 5.53.

The performance of the $\mathrm{HgI}_{2}$ Frisch collar device demonstrates the high quality of $\mathrm{HgI}_{2}$ tetragonal prismatic crystals grown with the low $\overline{\mathrm{M}}_{\mathrm{w}}$ polyethylene, implying that organic additives utilized in $\mathrm{HgI}_{2}$ growth with the Faile method did not pose detrimental effects to the crystal quality. The tetragonal prismatic shape of as-grown crystals is fortuitous as very 
Detector bias time at the beginning of counting is 36 hours

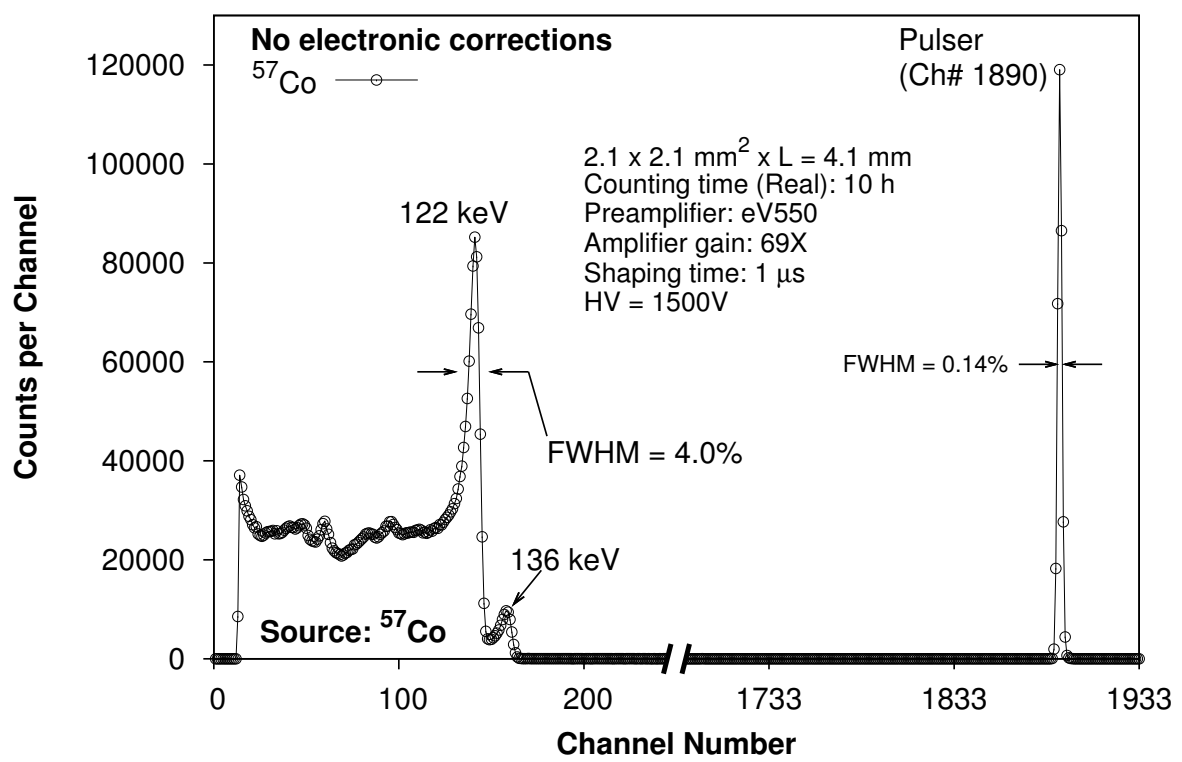

Figure 5.44. Pulse height spectrum taken with a $2.1 \times 2.1 \times 4.1 \mathrm{~mm}^{3} \mathrm{HgI}_{2}$ Frisch collar device being fully irradiated with a ${ }^{57} \mathrm{Co}$ gamma ray source positioned $3.0 \mathrm{~cm}$ away from the detector side. A 4.0\% FWHM energy resolution is achieved at $122 \mathrm{keV}$.

little post-growth processing was required (Section 4.9.1). Because $\mathrm{HgI}_{2}$ is a soft material, this minimal crystal handling also ensures the integrity of the detector materials, which appears to be another reason for good detector performance (as compared to the result in [27]). 


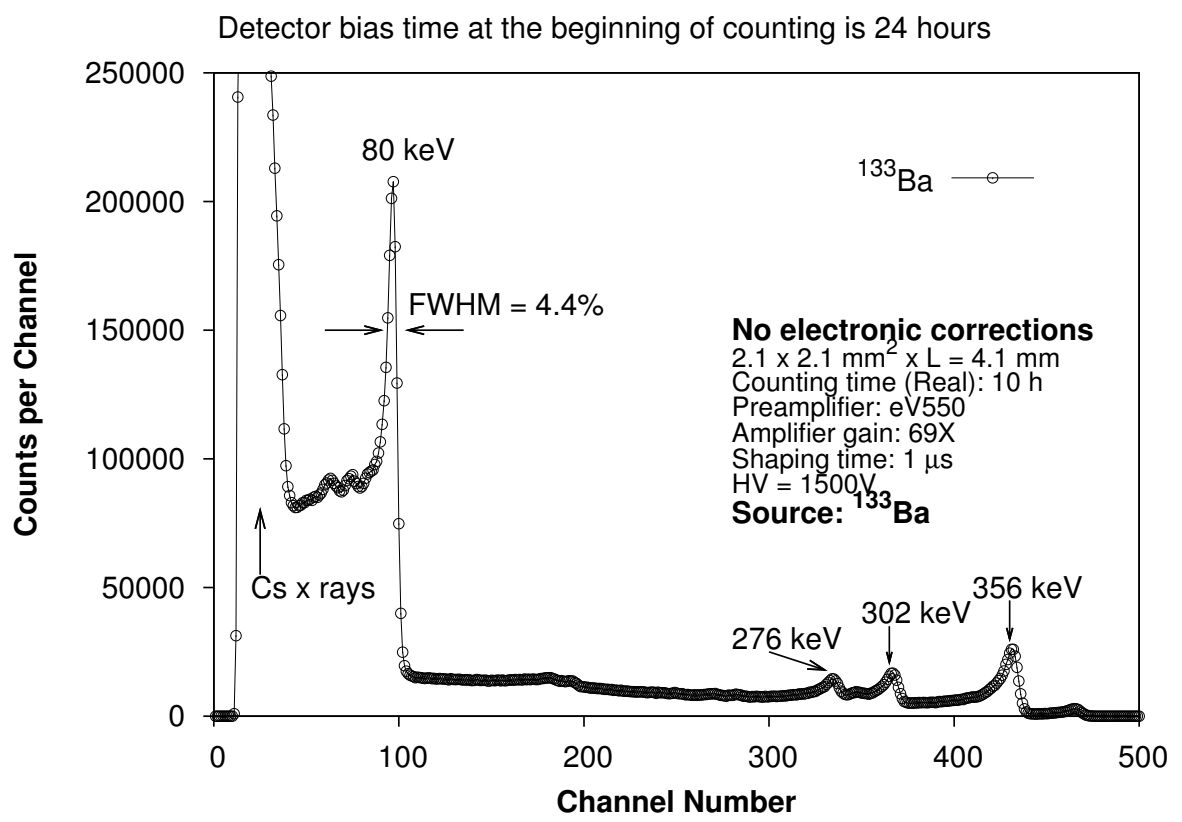

Figure 5.45. Pulse height spectrum taken with a $2.1 \times 2.1 \times 4.1 \mathrm{~mm}^{3} \mathrm{HgI}_{2}$ Frisch collar device being fully irradiated with a ${ }^{133} \mathrm{Ba}$ gamma ray source positioned $3.0 \mathrm{~cm}$ away from the detector side. A 4.4\% FWHM energy resolution is achieved at $80 \mathrm{keV}$.

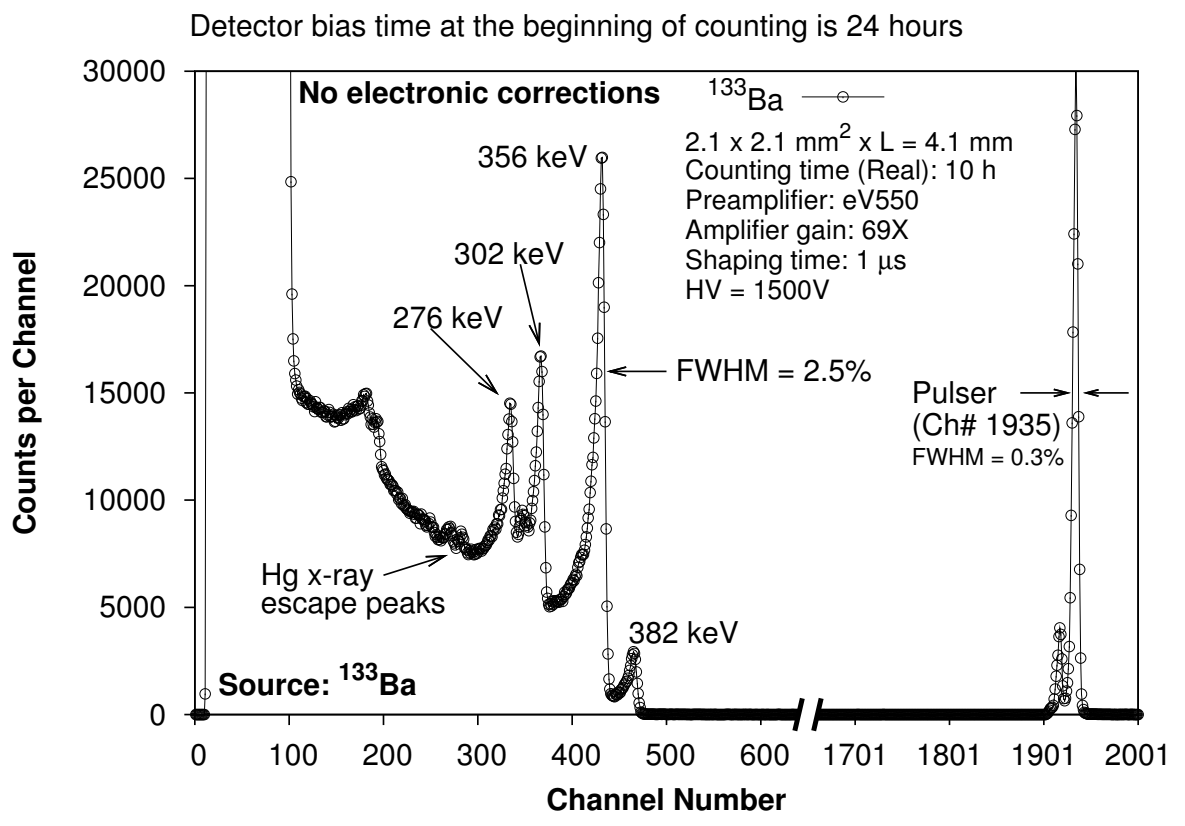

Figure 5.46. Expanded view of the 276, 302, 356, and $382 \mathrm{keV}$ full energy peaks of ${ }^{133} \mathrm{Ba}$ (see Fig. 5.45). A 2.5\% FWHM energy resolution is achieved at $356 \mathrm{keV}$. 


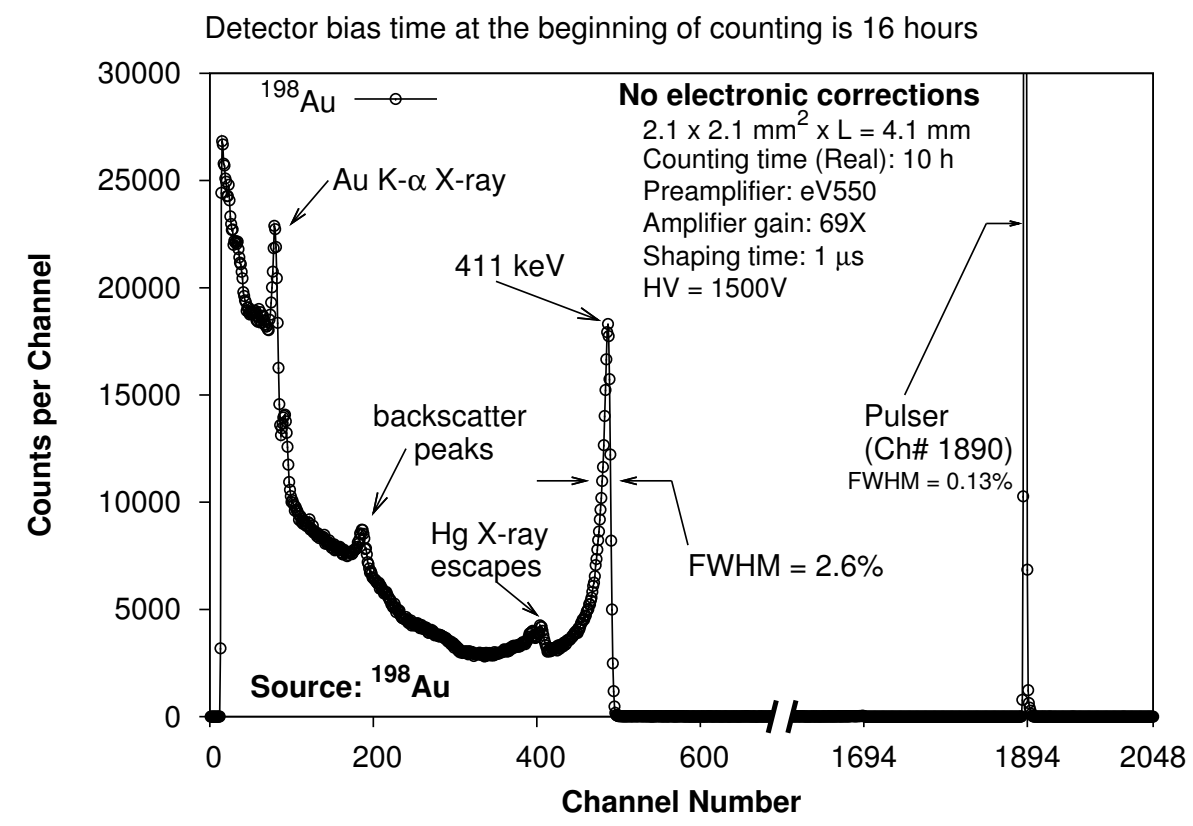

Figure 5.47. Pulse height spectrum taken with a $2.1 \times 2.1 \times 4.1 \mathrm{~mm}^{3} \mathrm{HgI}_{2}$ Frisch collar device being fully irradiated with a ${ }^{198} \mathrm{Au}$ gamma ray source positioned $3.0 \mathrm{~cm}$ away from the detector side. A $2.6 \%$ FWHM energy resolution is achieved at $411 \mathrm{keV}$.

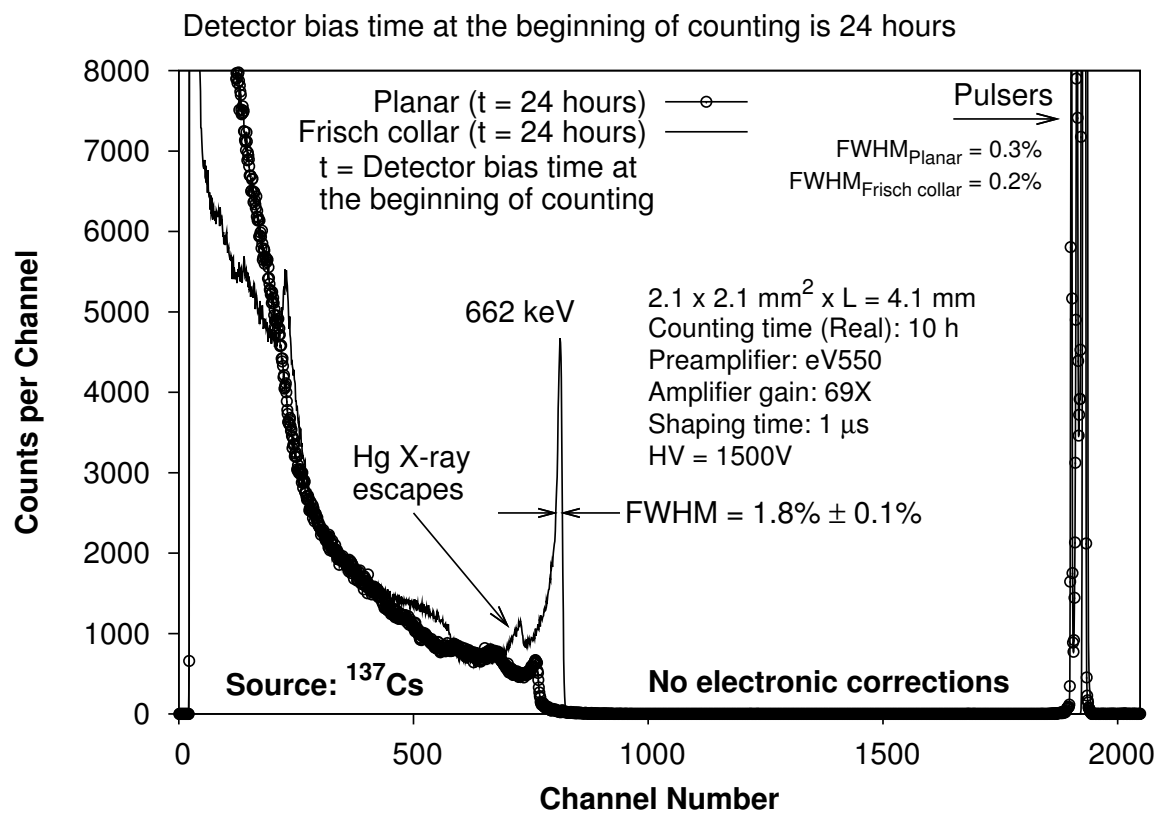

Figure 5.48. Pulse height spectra taken with a $2.1 \times 2.1 \times 4.1 \mathrm{~mm}^{3} \mathrm{HgI}_{2}$ device (Frisch collar and planar) being fully irradiated with a ${ }^{137} \mathrm{Cs}$ gamma ray source positioned $3.0 \mathrm{~cm}$ away from the detector side. A 1.8\% FWHM energy resolution is achieved at $662 \mathrm{keV}$.. 
Detector bias time at the beginning of counting is 24 hours

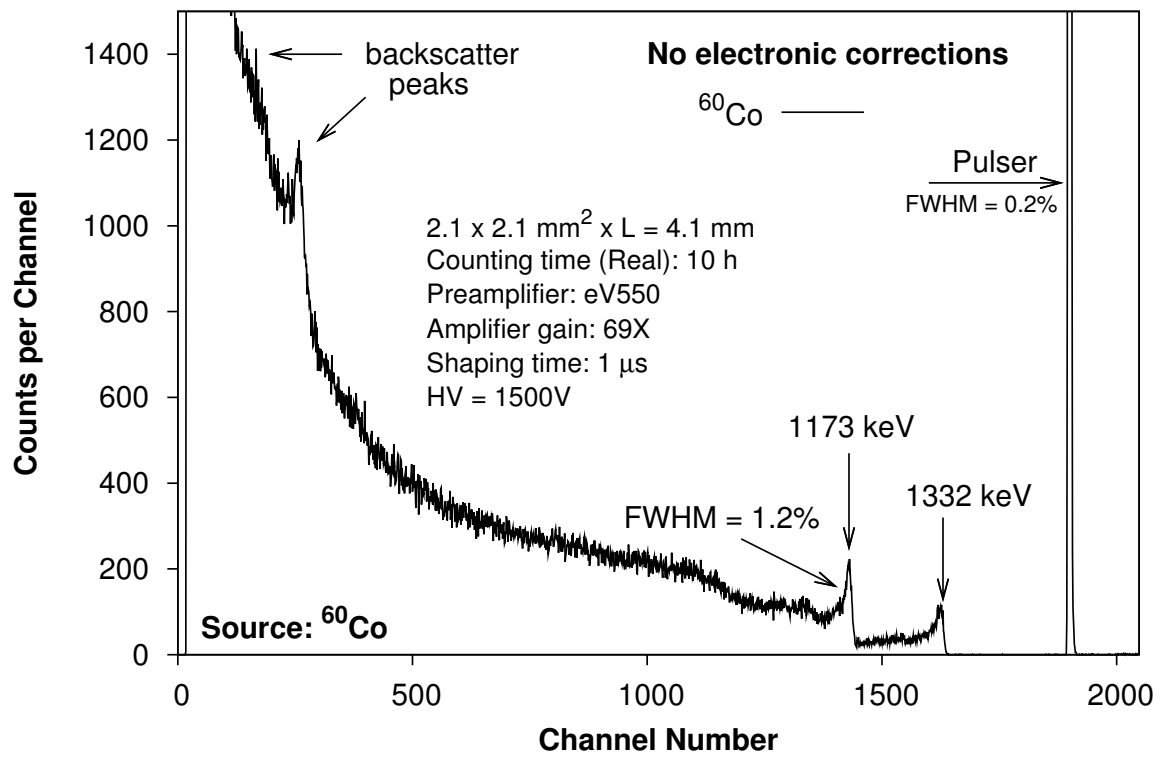

Figure 5.49. Pulse height spectrum taken with a $2.1 \times 2.1 \times 4.1 \mathrm{~mm}^{3} \mathrm{HgI}_{2}$ Frisch collar device being fully irradiated with a ${ }^{60} \mathrm{Co}$ gamma ray source positioned $3.0 \mathrm{~cm}$ away from the detector side. A $1.2 \%$ FWHM energy resolution is achieved at $1173 \mathrm{keV}$.

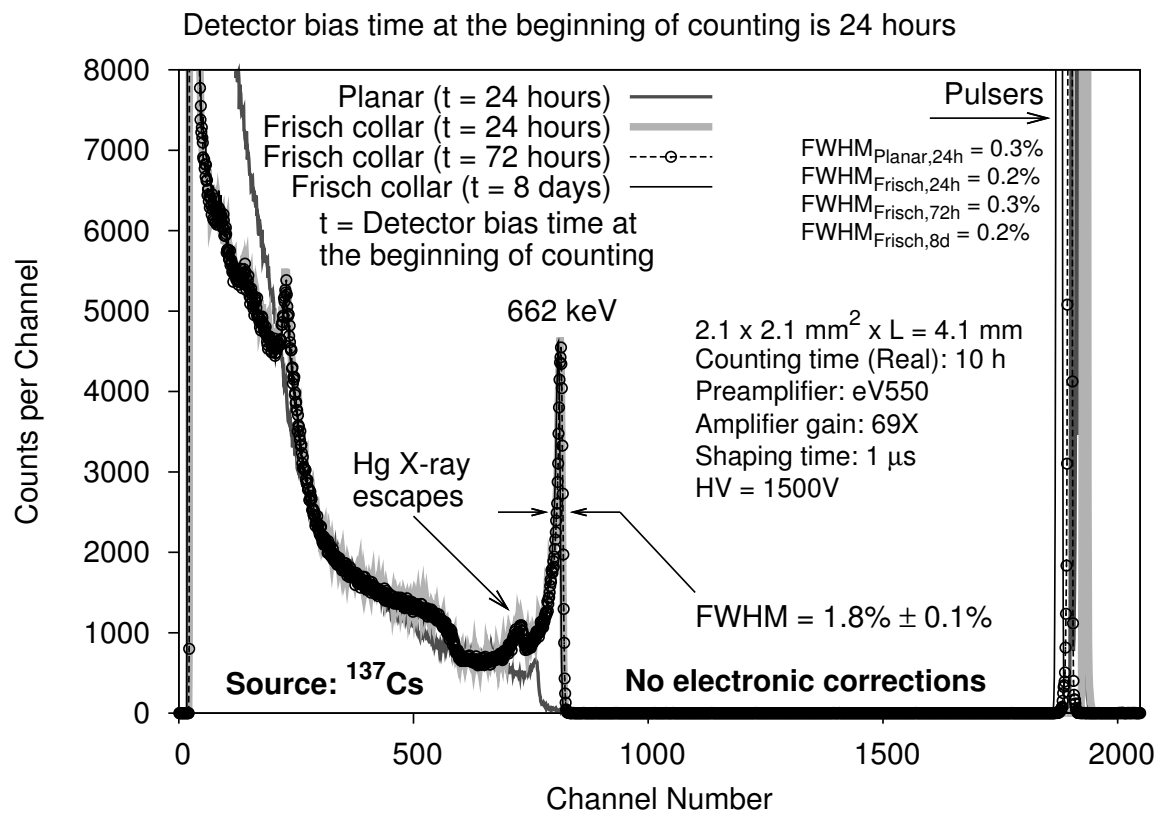

Figure 5.50. Pulse height spectra taken with a $2.1 \times 2.1 \times 4.1 \mathrm{~mm}^{3} \mathrm{HgI}_{2}$ device (Frisch collar and planar) being fully irradiated with a ${ }^{137} \mathrm{Cs}$ gamma ray source positioned $3.0 \mathrm{~cm}$ away from the detector side. A 1.8\% FWHM energy resolution is achieved at $662 \mathrm{keV}$. The detector was under bias for different periods of time before each pulse height spectrum was collected. 


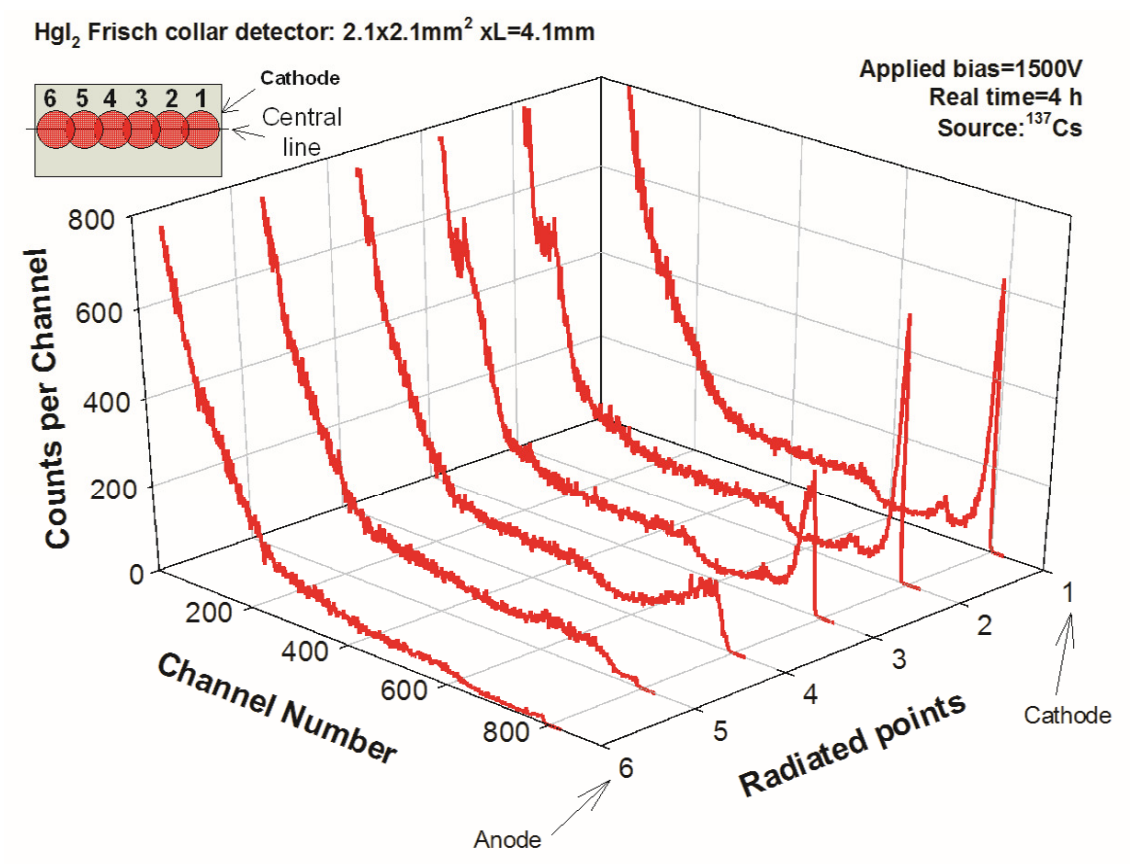

Figure 5.51. Pulse height spectra collected from a collimated ${ }^{137} \mathrm{Cs}$ gamma-ray source with the $2.1 \times 2.1 \times 4.1 \mathrm{~mm}^{3} \mathrm{HgI}_{2}$ Frisch collar device. The device was probed with a highly collimated gamma-ray source along the central line of the detector at points 1 to 6 at $1500 \mathrm{~V}$ for four hour real time at each point.

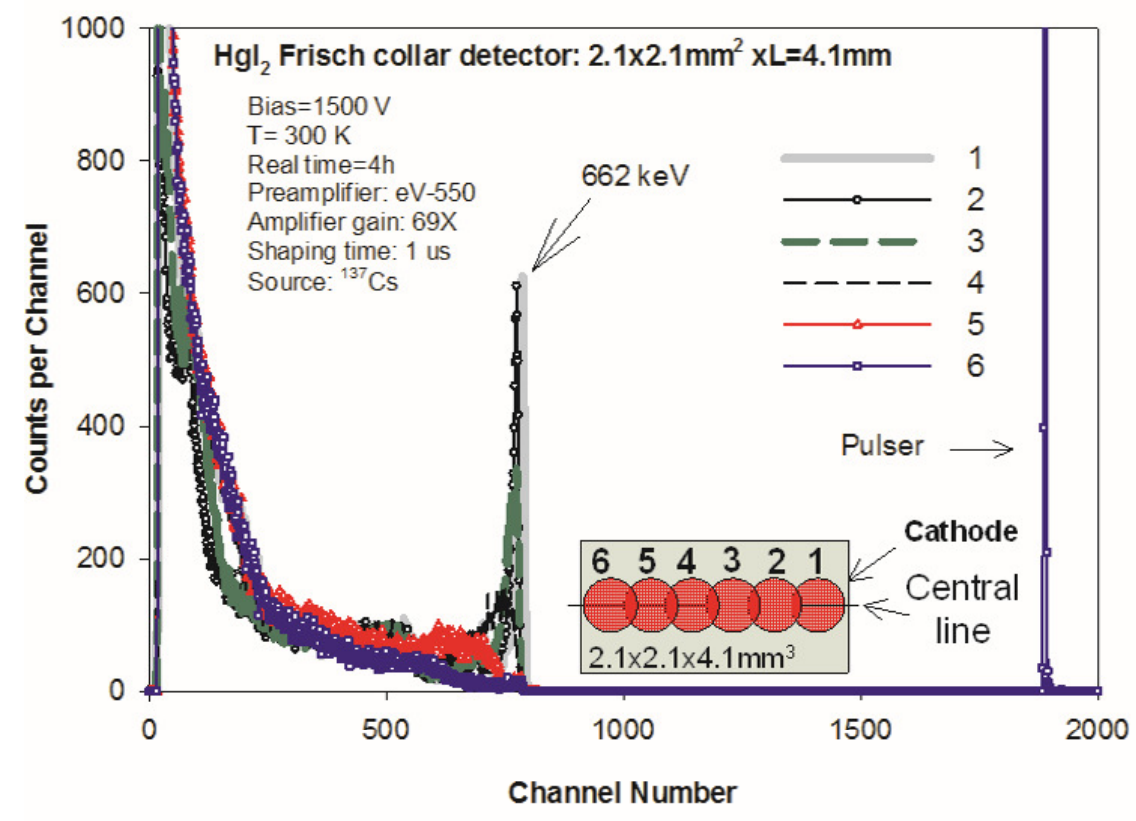

Figure 5.52. Pulse height spectra collected from a collimated ${ }^{137} \mathrm{Cs}$ gamma-ray source with the $2.1 \times 2.1 \times 4.1 \mathrm{~mm}^{3} \mathrm{HgI}_{2}$ Frisch collar device. 


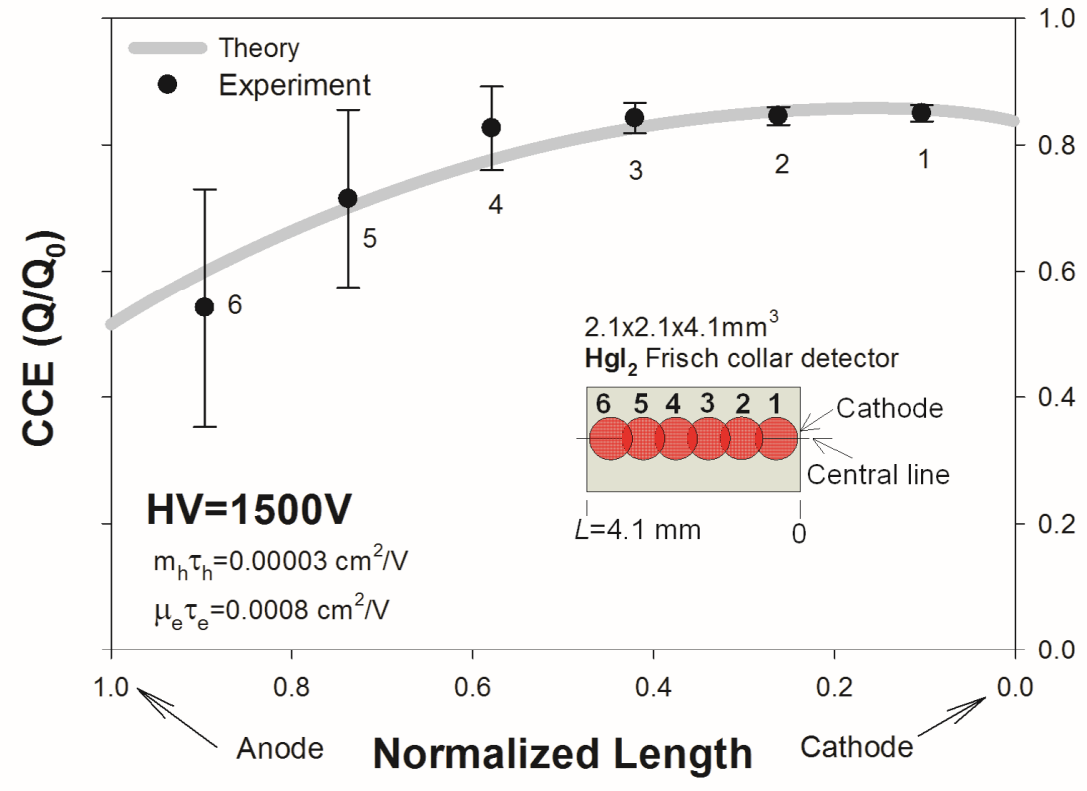

Figure 5.53. The CCE profile at $1500 \mathrm{~V}$ applied to the anode (collecting electrode) along the central line of the $2.1 \times 2.1 \times 4.1 \mathrm{~mm}^{3} \mathrm{HgI}_{2}$ Frisch collar device. The experimental data points are the normalized peak channel of the photopeak for the irradiated points ( 1 to 6) of the Frisch collar device. The error bars represent the photopeak FWHM at $662 \mathrm{keV}$. For the best CCE profile, the mobility-lifetime products $\mu_{e, h} \tau_{e, h}$ of $0.0008 \mathrm{~cm}^{2} \mathrm{~V}^{-1}$ and $0.00003 \mathrm{~cm}^{2} \mathrm{~V}^{-1}$ are estimated for the electrons and holes, respectively. 


\subsection{Observations of the Grown Crystals}

Two features of $\mathrm{HgI}_{2}$ are examined. First, the growth layers or striations on the $\{110\}$ faces are observed and analyzed in Section 5.9.1. Second, the morphology of the crystals are examined and compared with computer generated models.

\subsubsection{Growth layer: $\mathbf{H g I}_{2}$ grown without additive}

When the $\{110\}$ faces in $\mathrm{HgI}_{2}$ crystals grown without any additive are compared to the ones grown with an additive, especially with low $\overline{\mathrm{M}}_{\mathrm{w}}$ polyethylene, two different growth layer patterns or striations are observed. Fig. 5.54(a) shows a $\mathrm{HgI}_{2}$ grown in a vertical furnace (i.e., with no additive), where a $\{110\}$ face was recognized by its square-shaped striation pattern, where the angle between the (001) plane and the growth layer or striation edges conformed with approximately a $45^{\circ}$ (Fig. 5.54(b)).

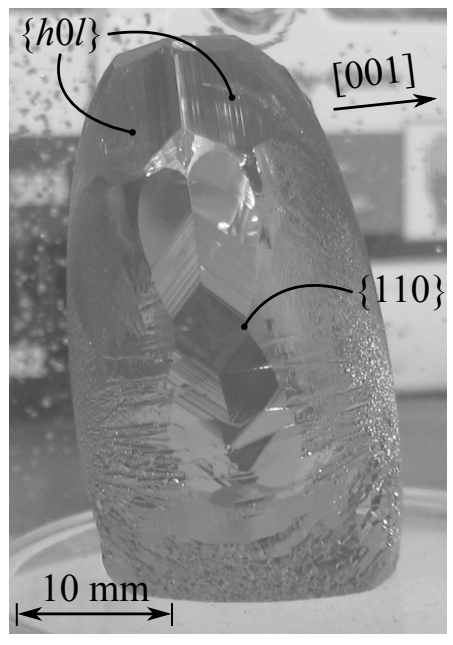

(a)

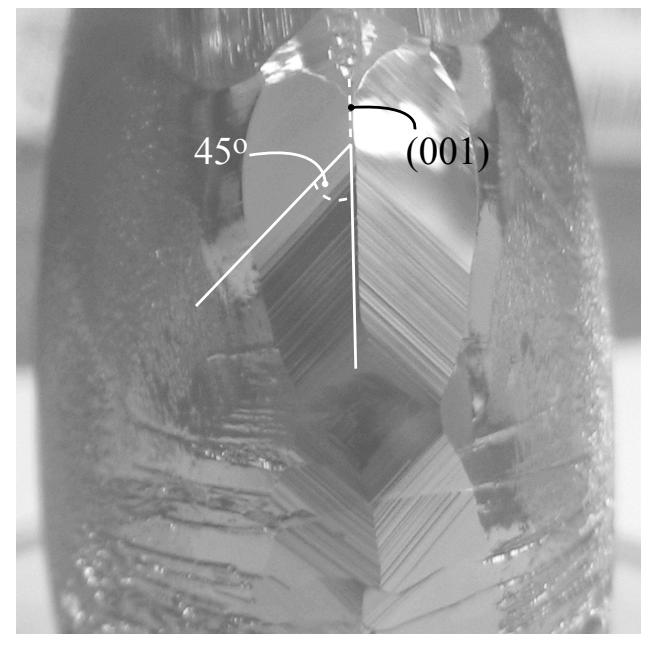

(b)

Figure 5.54. (a) $\{110\}$ face in a $\mathrm{HgI}_{2}$ crystal, with other identified faces and growth direction. (b) The angle between the (001) plane and the growth layer or striation edges was indeed approximately $45^{\circ}$.

$\mathrm{HgI}_{2}$ crystals grown in a horizontal furnace without any additive also displayed the same square-shaped growth layers or striations on the $\{110\}$ faces, as can be seen in Fig. 5.55(a). The [001] direction was found from cleaving the (001) planes off the crystal, 
and it was observed that the growth layer or striation edges in $\{110\}$ also formed approximately $45^{\circ}$ angle with the (001) (cleavage) plane (Fig. 5.55(b)), as previously observed in Fig. 5.54(b).

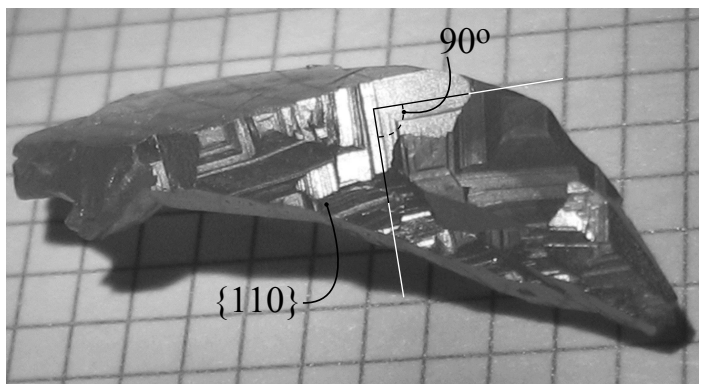

(a)

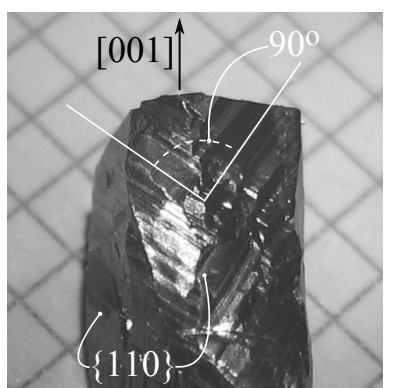

(b)

Figure 5.55. (a) $\mathrm{A} \mathrm{HgI}_{2}$ crystal grown with no additive, where square-shaped growth layers or striations observable in the $\{110\}$ faces. (b) Cleaving the crystal identifies the c-axis or the [001] direction, and the position of the striations relative to the (001) plane.

In crystallography, striations are understood to be produced by alternating growth of adjacent crystal faces [69]. Therefore, by finding what family of lines the striations belong to, the adjacent crystal face(s) can be determined. As observed in Fig. 5.55, the $\{110\}$ striations in $\mathrm{HgI}_{2}$ grown without any additive form approximately a $45^{\circ}$ angle with the (001) plane. For simplicity, determination of the striation-line was done based on Fig. 5.56(a), where the striations were represented by line $[h k l]$ forming a $45^{\circ}$ angle with a line parallel to [110]. Both lines, coplanar in the (110) plane, intersect a parallelepiped with $a=b=$ $4.361 \AA$ and $c=12.450 \AA$, or in other words, $\mathrm{HgI}_{2}$ unit cell. Thus, the line II to [ $\left.\overline{1} 10\right]$ has a magnitude or length of

$$
|[\overline{1} 10]|=\sqrt{((-1) \cdot a)^{2}+\left((1 \cdot a)^{2}\right)}=a \sqrt{2},
$$

and line $[h k l]$ is found by solving for $h, k$, and $l$, by trial and error, in

$$
\sqrt{\left(h^{2}+l^{2}\right) \cdot a^{2}+l^{2} \cdot c^{2}}=\frac{a \sqrt{2}}{\cos 45^{\circ}} .
$$

After $\mathrm{HgI}_{2}$ parameter values are substituted into Eq. 5.1, the appropriate $h, k$, and $l$ values are found to be $-1,1$, and $\frac{1}{2}$, respectively. These values, after being converted into integers, 
translate into line [221], which is coplanar with the (102) plane (Fig. 5.56(b)). ${ }^{1}$ Therefore, the striations in the $\{110\}$ faces on $\mathrm{HgI}_{2}$ crystals grown with no additive belong to the $\langle 221\rangle$ lines, which are coplanar with both the $\{110\}$ and $\{102\}$ planes. In other words, in $\mathrm{HgI}_{2}$ grown without any additive, the $\{110\}$ faces grow alternatingly with the $\{102\}$ faces.

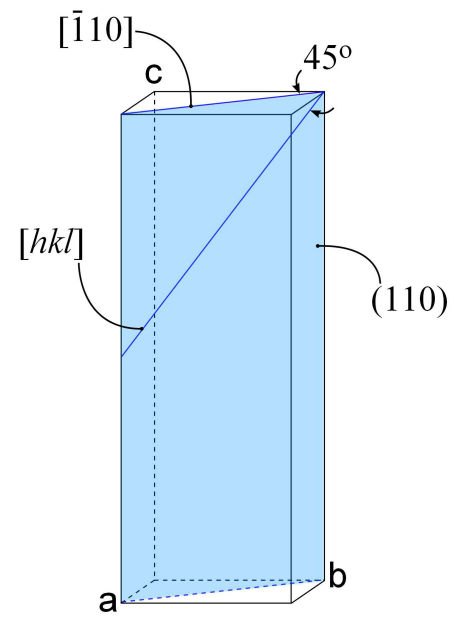

(a)

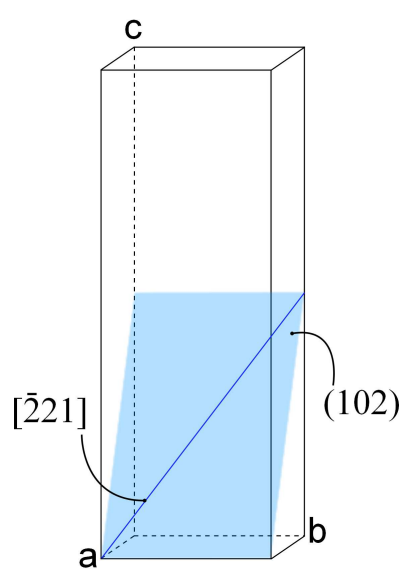

(b)

Figure 5.56. Determination of adjacent crystal face that produce striae on $\{110\}$. (a) Line $[h k l]$ forms a $45^{\circ}$ angle with a line II to [ $[110]$ with length $a \sqrt{2}$. (b) The line $[h k l]$ in (a) is found to be [2̄21], which is coplanar with both (110) and (102).

\footnotetext{
${ }^{1}$ The angle $[\overline{2} 21]$ made with $(001)$, or, more appropriately, [ $\left.\overline{1} 10\right]$, is obtained by subtituting $[h k l]=\left[\overline{1} 1 \frac{1}{2}\right]$ into Eq. 5.1, which results in $45.27^{\circ}$.
} 


\subsubsection{Growth layer: $\mathrm{HgI}_{2}$ grown with low $\overline{\mathrm{M}}_{\mathrm{w}}$ polyethylene}

As can be observed in Fig. 5.57(a), showing twin $\mathrm{HgI}_{2}$ crystals grown with low $\overline{\mathrm{M}}_{\mathrm{w}}$ polyethylene, the growth layers or striations in the $\{110\}$ faces are shaped more as rhombohedron than square. Details on the surface, as seen in Fig. 5.57(b), show that the edges of the growth layers or striations form approximately a $63^{\circ}$ angle with the (001) plane.

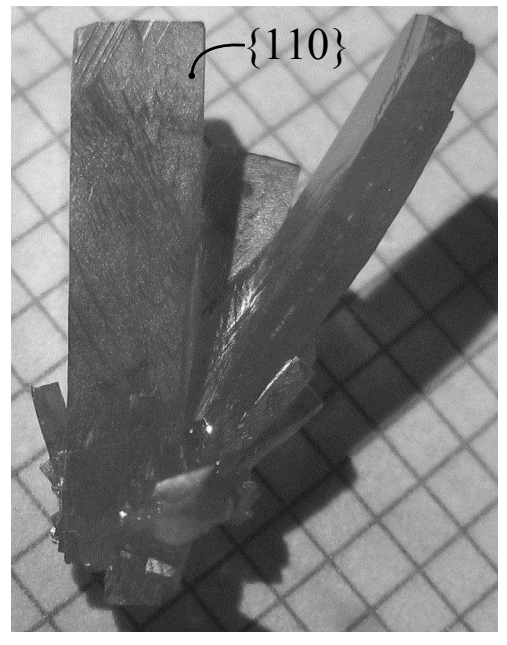

(a)

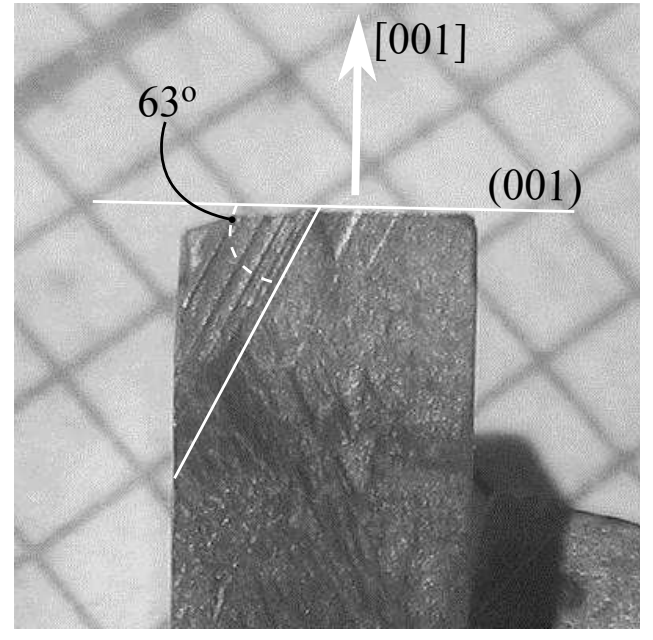

(b)

Figure 5.57. (a) $\mathrm{HgI}_{2}$ twin crystals grown with low $\overline{\mathrm{M}}_{\mathrm{w}}$ polyethylene in a horizontal furnace, showing rhombohedral-shaped growth layers or striations. (b) Edges of the growth layers or striations from approximately a $63^{\circ}$ angle with the (001) plane.

Again as in the previous analysis for $\mathrm{HgI}_{2}$ crystals grown without additive, determination of the striation-line was done based on Fig. 5.58(a), where the striations were represented by a line $[h k l]$ forming a $63^{\circ}$ angle with a line of length parallel to [1110] in the (110) plane and these lines intersect the $\mathrm{HgI}_{2}$ unit cell. The line [hkl] forming a $63^{\circ}$ angle with [110] is found by solving for $h, k$, and $l$, by trial and error, in

$$
\sqrt{\left(h^{2}+l^{2}\right) \cdot a^{2}+l^{2} \cdot c^{2}}=\frac{a \sqrt{2}}{\cos 63^{\circ}} .
$$

After $\mathrm{HgI}_{2}$ parameter values are substituted into Eq. 5.2, the appropriate $h, k$, and $l$ values are found to be $-1,1$, and 1 , respectively, which translate into line [1111], and thus the line is coplanar with the (101) plane (Fig. 5.58(b)). ${ }^{2}$ Therefore, the striations in the $\{110\}$ faces

\footnotetext{
${ }^{2}$ The angle [ $\left.\overline{1} 11\right]$ made with (001), or, more appropriately, [ $\left.\overline{1} 10\right]$, is obtained by subtituting $[h k l]=[\overline{1} 11]$
} 
on $\mathrm{HgI}_{2}$ crystals grown with low $\overline{\mathrm{M}}_{\mathrm{w}}$ polyethylene belong to the $\langle 111\rangle$ family of lines, which are coplanar with both the $\{110\}$ and $\{101\}$ family of planes. In other words, in $\mathrm{HgI}_{2}$ grown with low $\overline{\mathrm{M}}_{\mathrm{w}}$ polyethylene, the $\{110\}$ faces grow alternatingly with the $\{101\}$ faces.

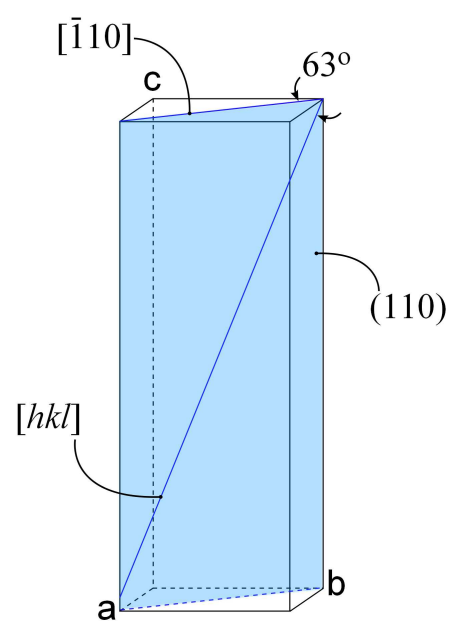

(a)

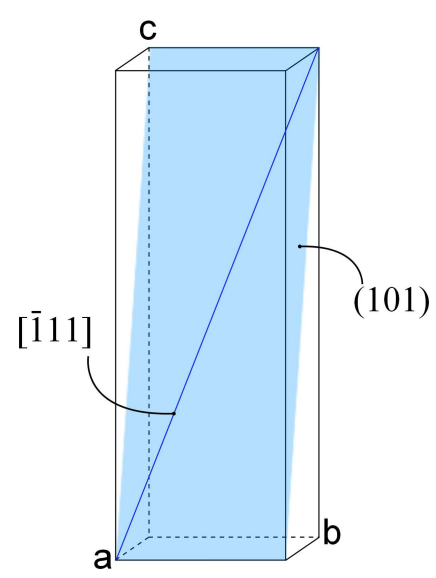

(b)

Figure 5.58. Determination of adjacent crystal face that produce striae on $\{110\}$ in $\mathrm{HgI}_{2}$ grown with low $\overline{\mathrm{M}}_{\mathrm{w}}$ polyethylene. (a) Line $[\mathrm{hkl}]$ forms approximately a $63^{\circ}$ angle with a line II to [110] with length $a \sqrt{2}$. (b) The line $[h k l]$ in (a) is found to be [111], which is coplanar with both (110) and (101).

into Eq. 5.2, which results in $63.65^{\circ}$. 


\subsubsection{Crystal morphology: computer-generated models}

As described in Chapter 3, according to the BFDH rules, the morphological importance of a crystal face, $M I_{(h k l)}$, is ascertained from its relative size and frequency of occurence, and is inversely proportional to its central distance, $r_{(h k l)}$, which is defined as the normal distance of the face to the center of the crystal. Fig. 5.59(a) shows a three-dimensional (3D) crystal model bounded by $\{001\}$ and $\{110\}$ crystal forms (i.e., family of crystal faces), with (001), (110) and (110) faces labeled. Central distances corresponding with the labeled faces, $r_{(001)}, r_{(1 \overline{1} 0)}$, and $r_{(110)}$, are defined in Fig. 5.59(b).

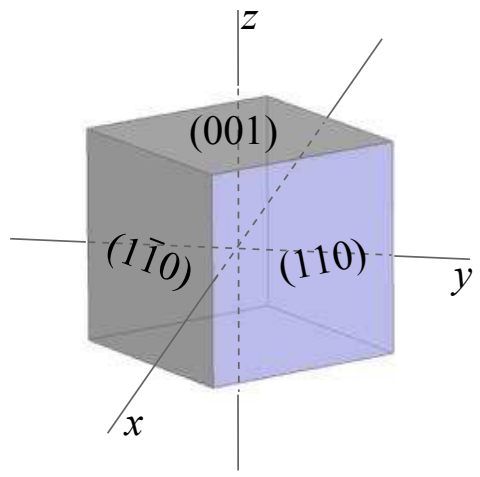

(a)

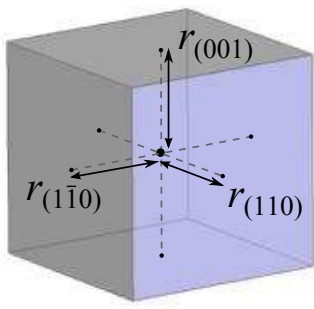

(b)

Figure 5.59. (a) A crystal model bounded by $\{001\}$ and $\{110\}$ crystal forms, with (001), (110) and (110) crystal faces labeled. (b) Definition of the central distance $r_{(h k l)}$ for the labeled crystal faces. The crystal model was created using WinXMorph [70, 71].

A few cases of growth with different values of $r_{(h k l)}$, using two-dimensional (2D) crystal models created using WinXMorph [70, 71], are listed in Table 5.8. These 2D crystal models are based on their respective 3D crystal models; for example, the crystal model in Fig. 5.60(a) is the 2D representation of the 3D crystal model in Fig. 5.59. To simplify crystal face identification, only faces in the positive quadrants are noted (Fig. 5.60(b)). Relevant inputs in WinXMorph for creation of these models are the crystal parameters $(a=b=$ 4.361( $\AA), c=12.450(\AA)$; no units are required as inputs) and point group $(4 / \mathrm{mm})$ of $\mathrm{HgI}_{2}$. Observations on $\mathrm{HgI}_{2}$ crystals show that, out of the faces predicted by the BFDH rules (Table 3.2), $\{001\},\{110\},\{101\}$ and/or $\{102\}$ occured most often and with significant sizes in vapor grown crystals, thus these faces, along with $\{103\}$, are used to create the crystal 
models.

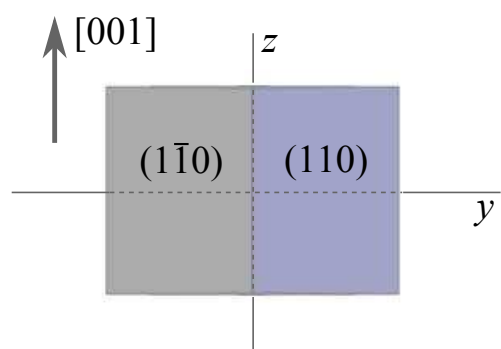

(a)

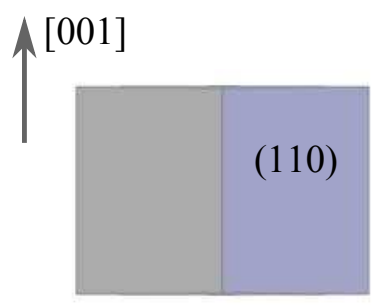

(b)

Figure 5.60. Notation for crystal face labels in Table 5.8. The models (labels excluded) were created using WinXMorph [70, 71].

A few assumptions are made in the creation of these crystal models. All crystal faces in the same crystal forms $\{h k l\}$ are assumed to be growing at the same growth rate, and the growth rate of any face $(h k l)$ is assumed to be constant over time $t$ (in arbitrary time units) and is defined as $\frac{\Delta r_{\{h k l\}}}{\Delta t}$ (with $r_{\{h k l\}}$ in arbitrary length units). For example, $r_{(1 \overline{1} 0)}$ $=r_{\{110\}}$, and $\frac{\Delta r_{\{110\}}}{\Delta t}=$ constant. These assumptions are obvious simplifications, because, first, growth factors such as the availability of growth nutrients and thermal conditions around the growing crystal, affect each crystal face individually. Each crystal face, even the ones belonging in the same crystal forms, has its own crystal growth rate, which may or may not be similar to the growth rates of other crystal faces. Depending on the growth rates of the neighboring faces, a crystal face that exists in a crystal nucleus may appear or disappear during the course of growth. The growth rate of one crystal face can also vary over time during the course of growth. Nevertheless, the simplifying assumptions do aid in placing the focus on the affects of $r_{\{h k l\}}$ and $\Delta r_{\{h k l\}}$ on crystal shapes.

The central distance of a crystal face at time $t$ can be derived as the following. Suppose $r_{\{h k l\}}$ at $t_{1}$ is known, then $r_{\{h k l\}}$ at $t_{2}$ is

$$
r_{\{h k l\}}\left(t_{2}\right)=r_{\{h k l\}}\left(t_{1}\right)+\frac{\Delta r_{\{h k l\}}}{\Delta t} \cdot\left(t_{2}-t_{1}\right)
$$


and to simplify, assuming that the observation is started at $t=0, r_{\{h k l\}}$ at any $\mathrm{t}$ is

$$
r_{\{h k l\}}(t)=r_{\{h k l\}}(0)+\frac{\Delta r_{\{h k l\}}}{\Delta t} \cdot(t)
$$

The above equations are reasonable to use for generating crystal models when the shape of the crystal nucleus or the values of $r_{\{h k l\}}$ are known. However, even without this information, and by recalling the definition of $M I_{h k l}$, the overall crystal shape can be used as an indicator of the growth rate of one crystal face relative to the rest of the faces. The ratio between the central distances turns out to be the factor that governs the appearance of certain crystal forms and the overall crystal shape. For example, the crystal in Fig. 5.61(a), with all $r_{\{h k l\}}=1$, and in Fig. 5.61(b), with all $r_{\{h k l\}}=2$, have the same crystal forms and overall crystal shape, because the ratio between the values of $r_{\{h k l\}}$ in Fig. 5.61(a) is the same as the ratio in Fig. 5.61(b). The same can be said for crystals in Figs. 5.61(c) and 5.61(d), both with the same crystal forms and shape because of the same ratio between $r_{\{h k l\}}$ values within each crystal.

The effect of $r_{\{h k l\}}$ ratio to crystal shape are shown in Cases $\mathbf{A}$ to $\mathbf{W}$ crystal models in Table 5.8, with $r_{\{h k l\}}$ values of either $1,1.5$ or 2 . In these crystal models, at least one $r_{\{h k l\}}$ is set to 1 , where the size of the corresponding crystal forms are larger than those with $r_{\{h k l\}}$ set to 1.5 or 2 . This occurence is clearly shown in Cases $\mathbf{A}$ to $\mathbf{E}$, where only one $r_{\{h k l\}}$ is set to 1 . Some of the crystal forms with $r_{\{h k l\}}=2$ are still observable (Cases $\mathbf{A}$ and $\mathbf{C}$ ), while others are not (Cases $\mathbf{D}$ and $\mathbf{E}$ ). Cases $\mathbf{B}$ to $\mathbf{E}$ essentially become Cases $\mathbf{F}$ to $\mathbf{I}$ when $r_{\{001\}}$ is set from 2 to 1.

Cases $\mathbf{J}$ to $\mathbf{L}$ are obtained by setting $r_{\{110\}}$ to 1 in Cases $\mathbf{C}$ to $\mathbf{D}$, respectively. Case $\mathbf{J}$ can also be obtained by setting $r_{\{101\}}$ to 1 in Case $\mathbf{B}$. The crystal models in Cases $\mathbf{J}$ to $\mathbf{L}$ are truncated, becoming bounded by $\{001\}$ as shown in Cases $\mathbf{M}$ to $\mathbf{O}$, when $r_{\{001\}}$ is set to 1 instead of 2. Case $\mathbf{A}$ becomes either one of Cases $\mathbf{P}$ to $\mathbf{S}$ when another $r_{\{h k l\}}$ is set to 1.5. Setting another $r_{\{h k l\}}$ to 1.5 in Case $\mathbf{B}$ alters the crystal shape into either Cases $\mathbf{T}, \mathbf{U}, \mathbf{V}$ or $\mathbf{W}$. All in all, the obvious results of altering the ratio of $r_{\{h k l\}}$ values are the increase or decrease in crystal form sizes and the appearance or disappearance of crystal 


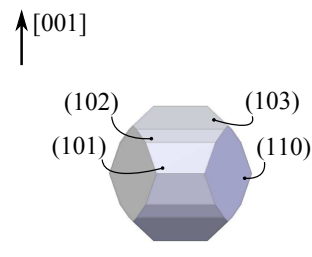

(a) all $r_{\{h k l\}}=1$

$r_{\{001\}}=1$

$r_{\{110\}}=1$

$r_{\{101\}}=1.25$

$r_{\{102\}}=1.25$

$r_{\{103\}}=1.25$

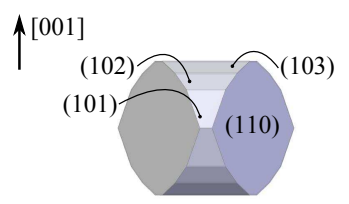

(c)

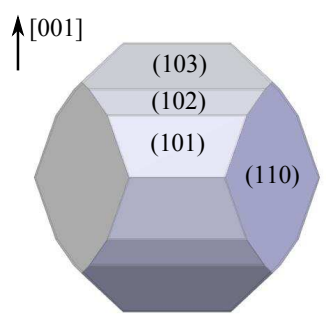

(b) all $r_{\{h k l\}}=2$

$r_{\{001\}}=2$

$r_{\{110\}}=2$

$r_{\{101\}}=2.5$

$r_{\{102\}}=2.5$

$r_{\{103\}}=2.5$

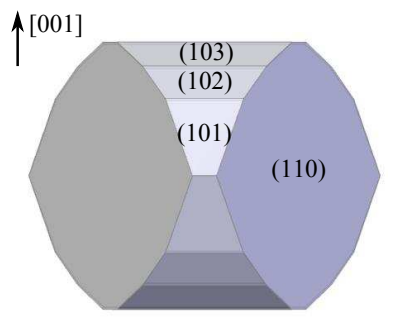

(d)

Figure 5.61. The effect of the ratio between the values of $r_{\{h k l\}}$ in governing crystal shape: All $r_{\{h k l\}}$ in either (a) or (b) have equal values, with all $r_{\{h k l\}}^{(\mathrm{a})}=1$ and all $r_{\{h k l\}}^{(\mathrm{b})}=2$ (i.e., the same ratio in either case), therefore, the results are both crystal models having the same crystal forms (i.e., family of crystal faces) and crystal shape. Both crystal models in (c) and (d) have the same ratio of $r_{\{h k l\}}$, hence, both crystal models also have the same crystal forms and shape..

forms, where crystal forms with the lowest value of $r_{\{h k l\}}$ are the bounding crystal forms. $\mathrm{HgI}_{2}$ crystals grown by vapor usually shaped as the variations of either Cases $\mathbf{A}$ or $\mathbf{B}$ (i.e., see other related Cases in Table 5.8), where $\{001\}$ or $\{110\}$ are the most prominent crystal faces, respectively. Observed crystal shapes and approximate crystal models are further described in Section 5.9.4. 
Table 5.8: $\mathrm{HgI}_{2}$ morphology models

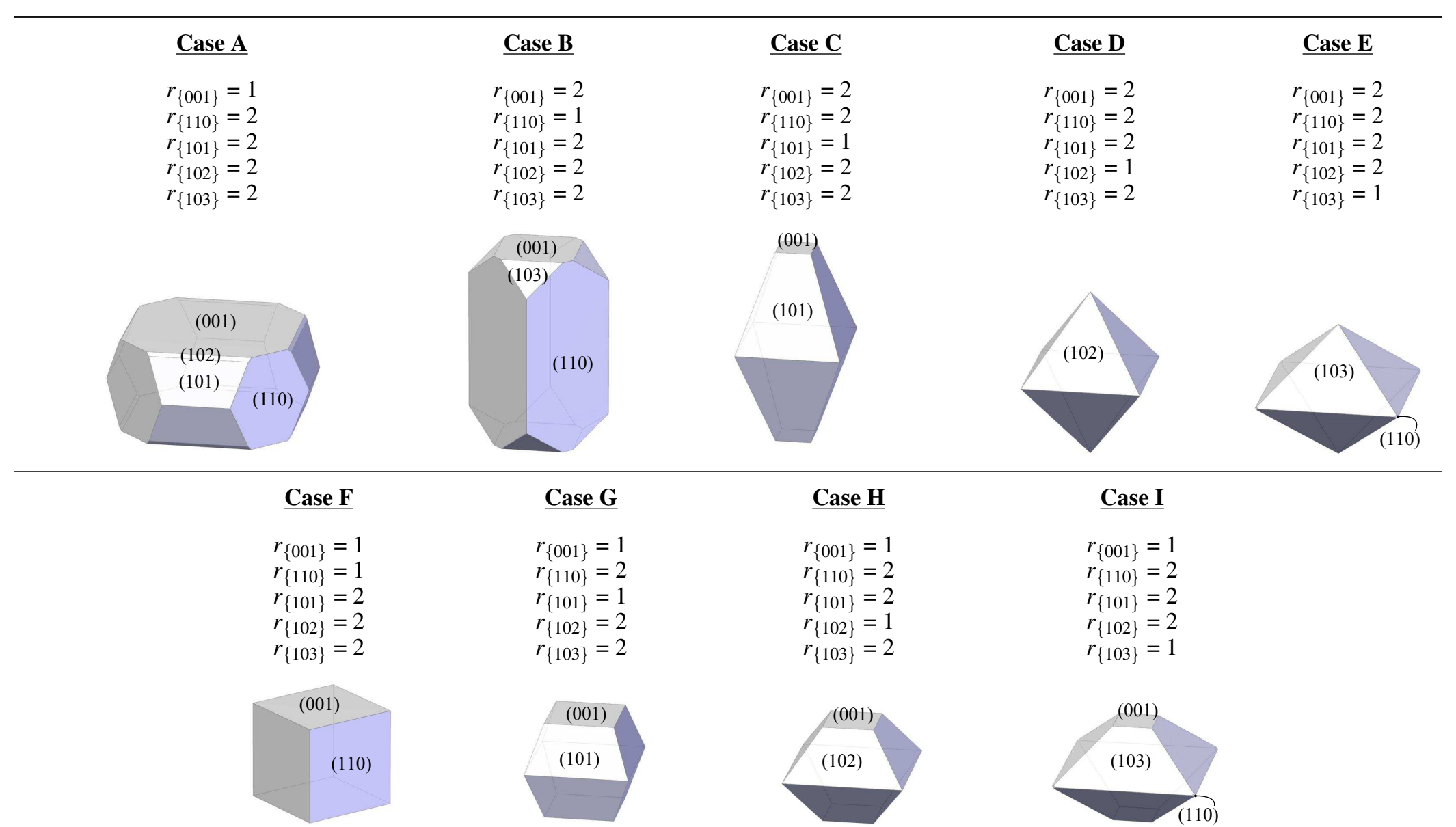


Table 5.8 Continued

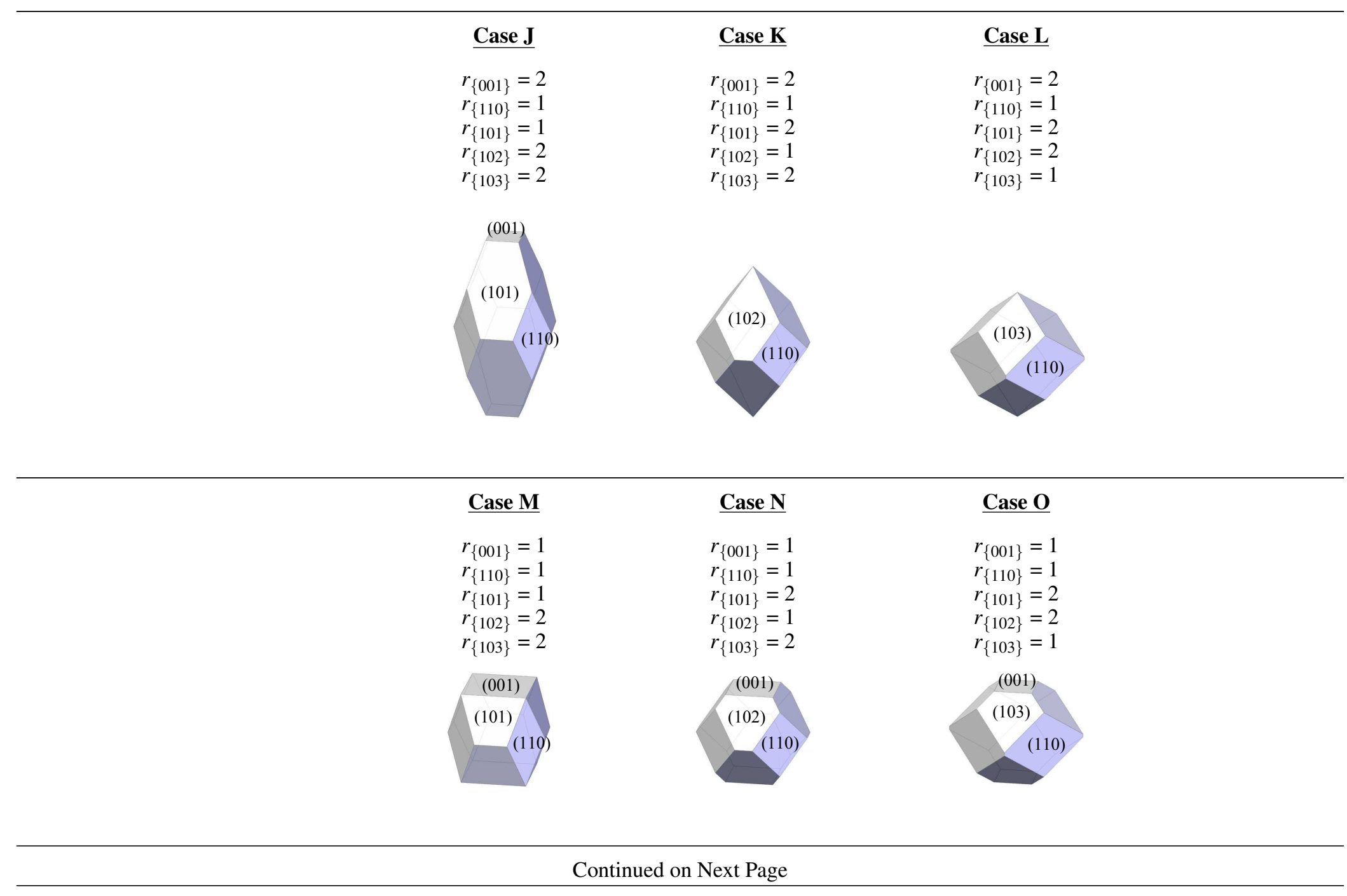


Table 5.8 Continued

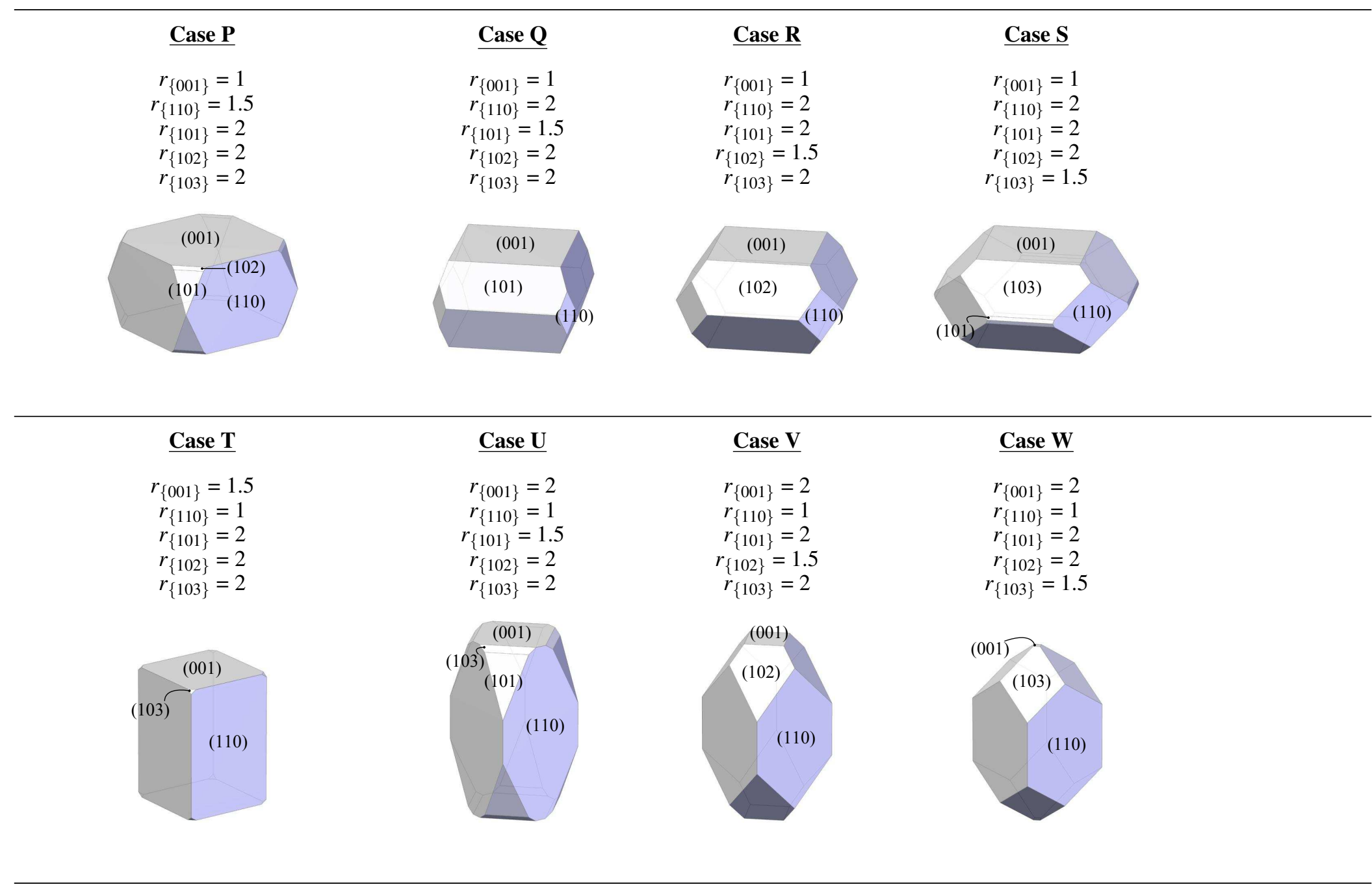


As mentioned previously, the ratio between the values of $r_{\{h k l\}}$ within a crystal is the factor that governs the presence or absence of certain crystal forms. The slowest growing crystal forms (i.e., crystal forms with the lowest (normal) growth rates) are the ones with the largest size and frequency of occurrence, eventually becoming the bounding crystal forms. Therefore, the ratio of (normal) growth rates of the observed crystal forms in one crystal can be deduced. As an illustration (Fig. 5.62), suppose at $t=0$ a crystal nucleus has the same $r_{\{h k l\}}=0.5$, but with $\Delta r_{\{001\}}=0.1$ and the rest of $\Delta r_{\{h k l\}}=0.3$. If the values of $\Delta r_{\{h k l\}}$ are assumed to be constant during growth, as $t$ progresses from 0 to 5 , the crystal shape in Case $\mathbf{A}$ is eventually obtained (Fig. 5.62(f)). Although $\Delta r_{\{110\}}=\Delta r_{\{101\}}=\Delta r_{\{102\}}$ $=\Delta r_{\{103\}}$, all of which are larger than $\Delta r_{\{001\}}$, eventually $\{102\}$ and $\{103\}$ become smaller or disappear from the crystal. Nevertheless, $\{001\}$ are comparatively larger than other faces, thus it can be inferred that $\{001\}$ has the lowest value of $\Delta r_{\{h k l\}}$ (as it is the case).

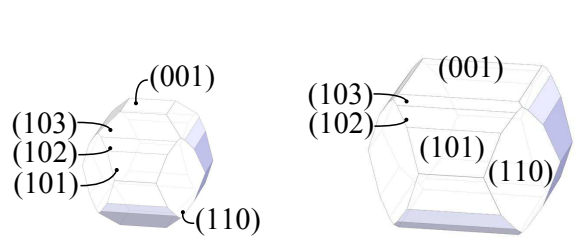

(a) $t=0$

(b) $t=1$

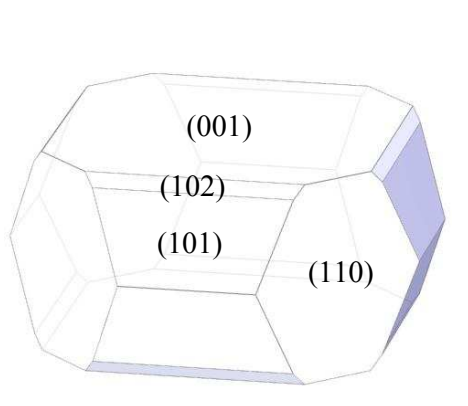

(e) $t=4$

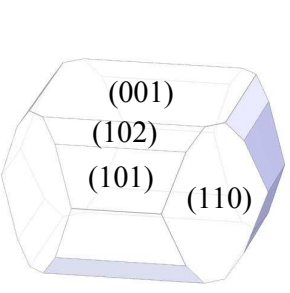

(c) $t=2$

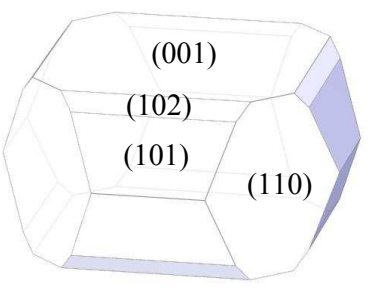

(d) $t=3$

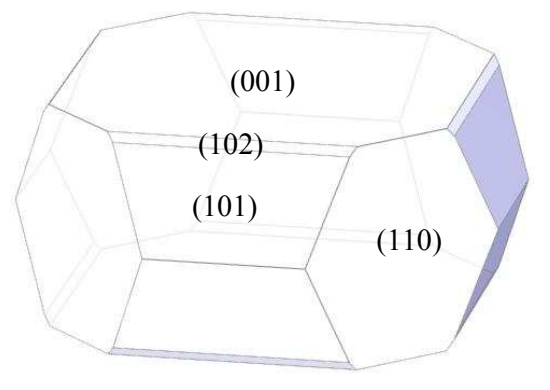

(f) $t=5$

Figure 5.62. Illustration on how $\Delta r_{\{h k l\}}$ may be implied from the presence of $\{h k l\}$. Eq. 5.4 is used to generate $r_{\{h k l\}}(t)$. The crystal models were created using WinXMorph. 


\subsubsection{Crystal morphology: observations on crystals}

Crystal models for several vapor grown $\mathrm{HgI}_{2}$ crystals are approximated and are shown in this section. A single crystal grown in a vertical furnace is shown Fig. 5.63, with a shape comparable to Case $\mathbf{A}$ in Table 5.8. The approximate crystal model (Fig. 5.63(c)) is created with WinXMorph with $r_{\{001\}}=3.6, r_{\{110\}}=8.4, r_{\{101\}}=9.4, r_{\{102\}}=8.4$, and $r_{\{103\}}=9.4$. These $r_{\{h k l\}}$ values, when normalized to $r_{\{001\}}$, which is the lowest value, becomes $r_{\{001\}}=$ $1, r_{\{110\}}=2.33, r_{\{101\}}=2.61, r_{\{102\}}=2.33$, and $r_{\{103\}}=2.61$. As observed, $\{001\}$ crystal forms have the largest area, thus presumably grow with the lowest $r_{\{h k l\}}$.

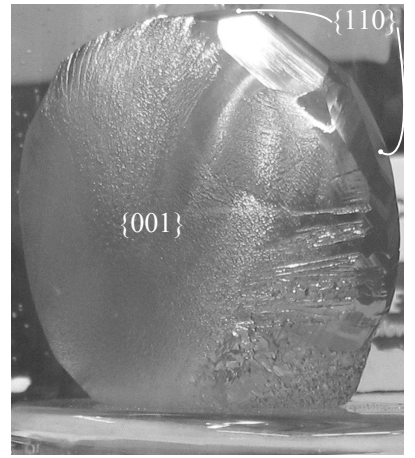

(a)

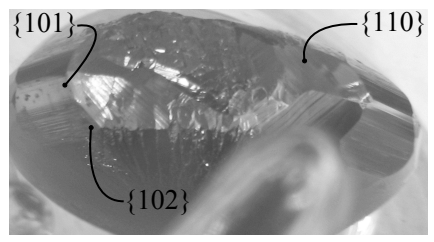

(b)

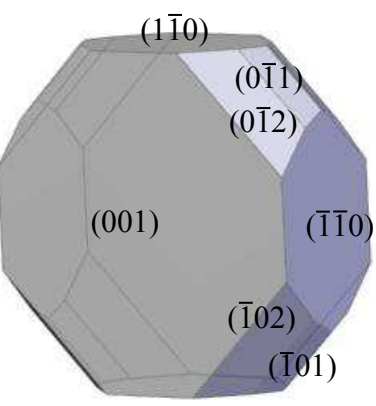

(c)

Figure 5.63. (a)-(b) The faces of a crystal, which are also shown in Fig. 5.54, can be determined from the striations in $\{110\}$. (c) A crystal model created using WinXMorph, with $r_{\{h k l\}}$ values listed in the text.

A small single crystal (Fig. 5.64(a)), grown in a horizontal furnace and without any additive, also has a crystal shape similar to the one grown in a vertical furnace, and its crystal model (Fig. 5.64(b)) is created using WinXMorph with $r_{\{001\}}=2.6, r_{\{110\}}=6.2$, $r_{\{101\}}=8, r_{\{102\}}=6.8$, and $r_{\{103\}}=6.8$. These $r_{\{h k l\}}$ values, when normalized to $r_{\{001\}}$, which is the lowest value, becomes $r_{\{001\}}=1, r_{\{110\}}=2.38, r_{\{101\}}=3.08, r_{\{102\}}=2.62$, and $r_{\{103\}}=2.62$. Similarly as observed in the case of $\mathrm{HgI}_{2}$ crystal grown in a vertical furnace, the crystal model can be approximated by Case $\mathbf{A}$ (with adjustments in some $r_{\{h k l\}}$ values), where the $\{001\}$ crystal forms have the largest area, thus presumably grow with the lowest $r_{\{h k l\}} \cdot$

Another single crystal (Fig. 5.65(a)), also grown in a horizontal furnace and without 


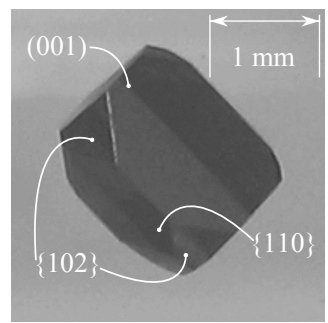

(a)

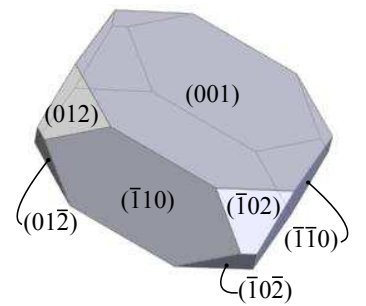

(b)

Figure 5.64. (a) A small single crystal from a growth without any additive in a horizontal furnace. (b) A crystal model created using WinXMorph, with $r_{\{h k l\}}$ values listed in the text.

any additive, shows a slightly different crystal shape and the approximated crystal model resembles Case $\mathbf{T}$, which is a variation of Case $\mathbf{B}$. The model is created using WinXMorph with most of $r_{\{001\}}=5, r_{\{110\}}=3.2, r_{\{101\}}=8, r_{\{102\}}=6.2$, and $r_{\{103\}}=6.2$. Larger $\{110\}$, $\{101\}$ and $\{102\}$ faces have the following $r_{\{h k l\}}$ values: $r_{(110)}=1.2, r_{(\overline{1} 10)}=2.25, r_{(101)}=$ $3.5, r_{(102)}=4.1, r_{(012)}=4.3, r_{(01 \overline{2})}=4.8$, and $r_{(10 \overline{2})}=4.1$. All $r_{\{h k l\}}$ values are normalized to $r_{(110)}$, which is the smallest, and are listed in Fig. 5.65.

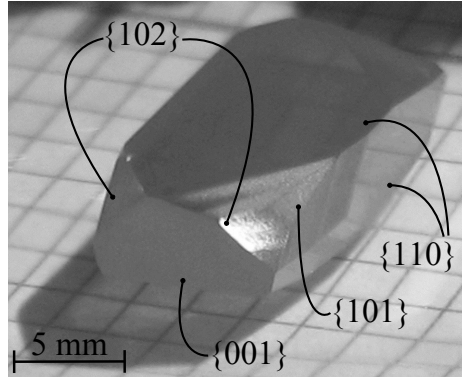

(a)

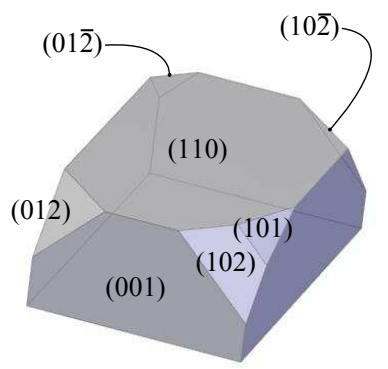

(b)

$$
\begin{array}{lllllll}
\hline \boldsymbol{r}_{(h k l)} \text { for }(\mathbf{b}) & & & & & & \\
\hline r_{(001)}=4.2 & r_{(00 \overline{1})}=4.2 & & & & \\
r_{(110)}=1.0 & r_{(101)}=2.9 & r_{(011)}=6.7 & r_{(102)}=3.4 & r_{(012)}=3.6 & r_{(103)}=6.7 & r_{(013)}=6.7 \\
r_{(\overline{1} 10)}=2.7 & r_{(\overline{1} 01)}=6.7 & r_{(0 \overline{1} 1)}=6.7 & r_{(\overline{1} 02)}=5.2 & r_{(0 \overline{1} 2)}=5.2 & r_{(\overline{1} 03)}=6.7 & r_{(0 \overline{1} 3)}=6.7 \\
r_{((1 \overline{1} 0)}=2.7 & r_{(10 \overline{1})}=6.7 & r_{(01 \overline{1})}=6.7 & r_{(10 \overline{2})}=3.4 & r_{(01 \overline{2})}=4.0 & r_{(10 \overline{3})}=6.7 & r_{(01 \overline{3})}=6.7 \\
r_{(\overline{1} \overline{1} 0)}=1.9 & r_{(\overline{1} \overline{1} \overline{1})}=6.7 & r_{(0 \overline{1} \overline{1})}=6.7 & r_{(\overline{1} 0 \overline{2})}=5.2 & r_{(0 \overline{1} \overline{2})}=5.2 & r_{(\overline{1} 0 \overline{3})}=6.7 & r_{(0 \overline{1} \overline{3})}=6.7
\end{array}
$$

Figure 5.65. (a) Another single crystal from a growth without any additive in a horizontal furnace. (b) A crystal model created using WinXMorph, with $r_{(h k l)}$ values listed above. Faces with the lowest values of $r_{\{h k l\}}$ compared to other faces in the same crystal forms $\{h k l\}$ are visible in the crystal morphology.

Two other examples of crystals grown without any additive in a horizontal furnace 
are shown in Figs. 5.67 and 5.69, while their positions or orientations in the ampoule can be assessed in Fig. 5.66. Although the shape of these crystals and the facets are not trivial to model with WinXMorph, their crystal faces can still be identified and their approximate crystal shapes (without faceting), along with their relative growth rates, can still be deduced. On one of the crystals, a symmetric or butterfly twin crystal, growth layers or striations indicative of $\{110\}$ are identified, as shown in Fig. 5.67(a). The details of the $\{110\}$ face, along with the bottom face, which is the crystal face against the ampoule wall, are shown in Fig. 5.67(b). Observation of the crystal position or orientation in the growth ampoule (top right in Fig. 5.66) shows that this particular $\{110\}$ face grows toward the ampoule wall. The existence of a twin boundary, though apparent in the opposite side of the $\{110\}$ face (Fig. 5.67(c)), is discovered after finding the (001) cleavage plane through cleaving one of the individual crystals (Fig. 5.68(b)).

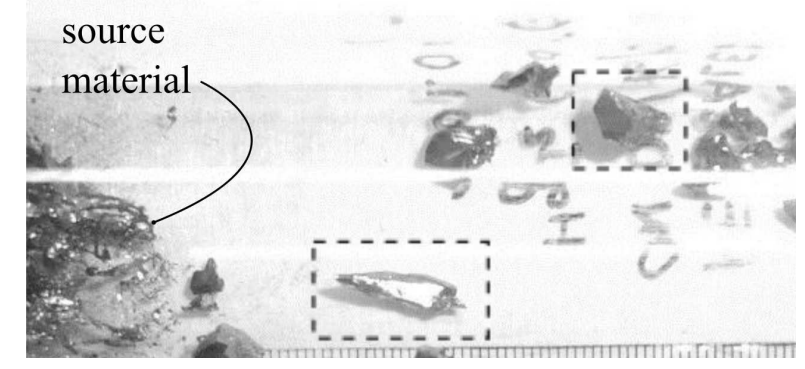

Figure 5.66. The position and orientation of the crystals in Figs. 5.67 and 5.69 in the growth ampoule.

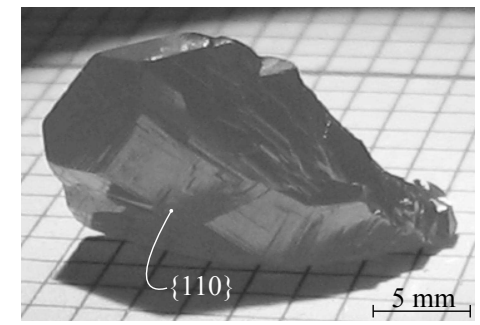

(a)

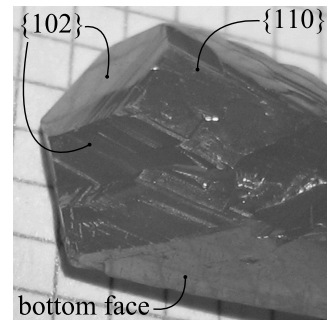

(b)

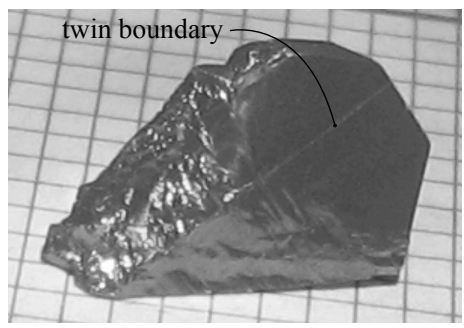

(c)

Figure 5.67. (a) A face belonging to $\{110\}$ is recognized by growth layers or striations. (b) Details of the growth layers on $\{110\}$ faces. (c) The opposite side of (a), where the twin boundary is visible.

The angle between the twin boundary with the (001) plane is around $40^{\circ}$, and with 
the assumption that the twin boundary is the intersection of a twin plane and the crystal face, the twin plane is found to belong to $\{103\}$. According to WinXMorph's calculation, the angle between the (001) and (103) planes is $43.58^{\circ}$. The visible striations and their orientation with respect to the (001) plane, which indicate that $\{102\}$ and $\{101\}$ faces grow alternatingly, also suggest that the opposite side is a $\{110\}$ face as well. A crystal model for the bottom half of the twin crystal is created using WinXMorph, where the values of $r_{\{h k l\}}$ normalized by two (the lowest value of $r_{\{h k l\}}$ ) are listed in Fig. 5.68. The approximate crystal model is a variation of Case $\mathbf{B}$ in Table 5.8, where $r_{\{110\}}$ are the lowest compared to other $r_{\{h k l\}}$, thus the size of $\{110\}$ are the largest. The value of $r_{\{10 \overline{3}\}}$ is set to the lowest value to approximate the twin plane.

The growth layers and the striations on $\{110\}$ suggest that $\Delta r_{\{102\}}$ is higher than $\Delta r_{\{110\}}$, but lower than other $\Delta r_{\{h k l\}}$, allowing $\{110\}$ and $\{102\}$ to grow alternatingly. Noticeable from the orientation of the crystal in Fig. 5.66, on the one hand, the face in Fig. 5.67(c) is exposed to more growth nutrients, being the face that grows away from the ampoule's wall, thus the growth layers appear to grow fast and less faceted. On the other hand, the more prismatic $\{110\}$ face in Fig. 5.67(a), with the face growing towards the ampoule's wall and, therefore, it is not exposed to much of the growth nutrients, allowing for a slower and faceted growth.

Fig. 5.69 shows another twin crystal (also shown in Fig. 5.55(a)) that was grown in a horizontal furnace without any additive, with its position and orientation in the growth ampoule before retrieval are shown in Fig. 5.66 (bottom left). The biggest individual crystal is bounded by $\{110\}$ faces, as indicated by the striations observed in Figs. 5.69(a)-(e), while the bottom face (the crystal face growing against the ampoule's wall) is a $\{102\}$ face. As in the previous twin crystal (Fig.5.67), the $\{110\}$ faces growing close or toward the ampoule's wall do not see much growth nutrients, resulting in slower growth that leads to larger and more prismatic or faceted crystal faces. The cleaved crystal (also shown in Fig. 5.55(b)) reveals that the apparent fast growth direction is the [001] direction. $\{102\}$ and $\{110\}$ crystal faces intersecting the (001) plane are recognizable and confirm the apparent crystal faces (Fig. 5.69(f)). Similar to earlier observations, the size of the $\{110\}$ faces in Figs. 5.69 


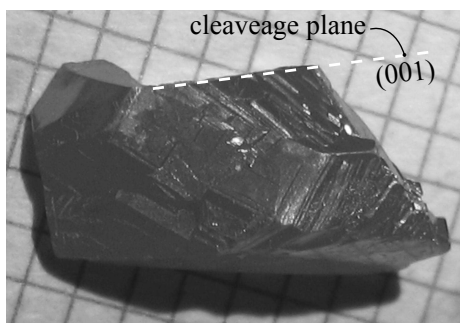

(a)

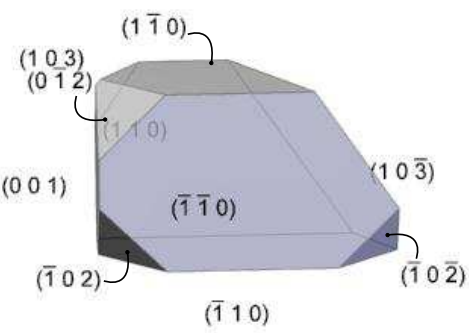

(c)

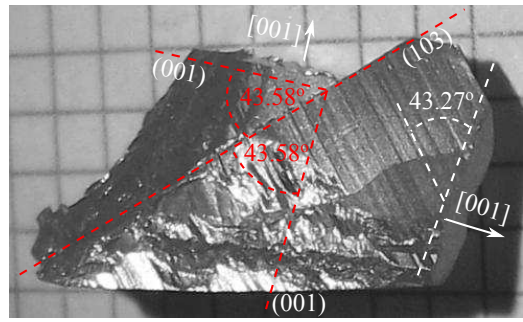

(b)

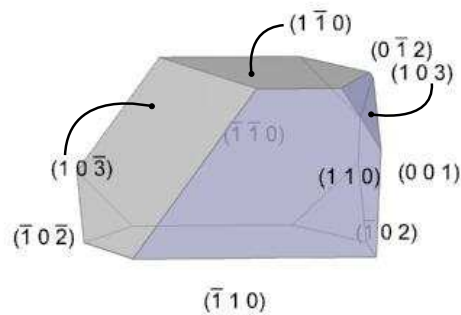

(d)

\begin{tabular}{lllllll}
\hline $\boldsymbol{r}_{(h k l)}$ for $(\mathbf{c}) \boldsymbol{\&}(\mathbf{d})$ & & & & & & \\
\hline$r_{(001)}=2.5$ & $r_{(00 \overline{1})}=3$ & & & & & \\
$r_{(110)}=1$ & $r_{(101)}=3.5$ & $r_{(011)}=3.5$ & $r_{(102)}=3.5$ & $r_{(012)}=3.5$ & $r_{(103)}=2.5$ & $r_{(013)}=3.5$ \\
$r_{(\overline{1} 10)}=1.5$ & $r_{(\overline{1} 01)}=3.5$ & $r_{(0 \overline{1} 1)}=3.5$ & $r_{(\overline{1} 02)}=2.25$ & $r_{(0 \overline{1} 2)}=2.25$ & $r_{(\overline{1} 03)}=3$ & $r_{(0 \overline{1} 3)}=3$ \\
$r_{(1 \overline{1} 0)}=1.5$ & $r_{(10 \overline{1})}=3.5$ & $r_{(01 \overline{1})}=3.5$ & $r_{(10 \overline{2})}=3.5$ & $r_{(01 \overline{2})}=3.5$ & $r_{(10 \overline{3})}=1$ & $r_{(01 \overline{3})}=3$ \\
$r_{(\overline{1} \overline{1} 0)}=1$ & $r_{(\overline{1} 0 \overline{1})}=3.5$ & $r_{(0 \overline{1} \overline{1})}=3.5$ & $r_{(\overline{1} 0 \overline{2})}=2.25$ & $r_{(0 \overline{1} \overline{2})}=3.5$ & $r_{(\overline{1} 0 \overline{3})}=3$ & $r_{(0 \overline{1} \overline{3})}=3$ \\
\hline
\end{tabular}

Figure 5.68. Crystal models for the bottom half of the crystal in Fig. 5.67. (a) The (001) cleavage plane for one individual is found. (b) The twin plane is found to be a $\{103\}$ plane, by comparing the observed angle between the (001) plane and the twin boundary with the calculated angles between the (normals of) (001) plane and other possible planes (c) A crystal model comparable to (a). (b) The opposite side of (d), comparable to (b).

implies that the values of $\Delta r_{\{110\}}$ are the lowest compared to other crystal faces, while the striations suggest that $\Delta r_{\{102\}}$ is higher than $\Delta r_{\{110\}}$, but lower than other $\Delta r_{\{h k l\}}$. The fast growth direction, as mentioned previously, is in the [001] direction, suggesting that $\Delta r_{\{001\}}$ is larger compared to either $\Delta r_{\{110\}}$ or $\Delta r_{\{102\}}$.

Besides a small single crystal in Fig. 5.64, $\{110\}$ faces in the single and twin crystals grown without any additive in horizontal furnaces (Figs. 5.65 to 5.69) is larger than the $\{001\}$, suggesting that the growth of $\mathrm{HgI}_{2}$ crystals in horizontal furnaces favors slower growth of $\{110\}$ compared to $\{001\}$. In addition, the apparent fast growth direction in the twin crystals is indeed the [001] direction, regardless the crystallographic orientation of the 


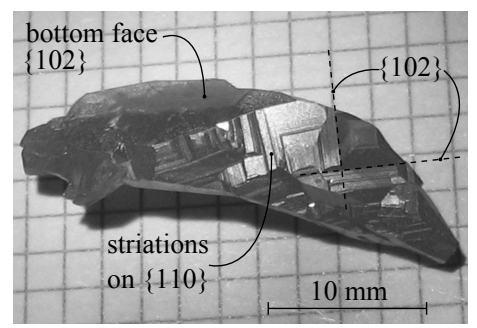

(a)

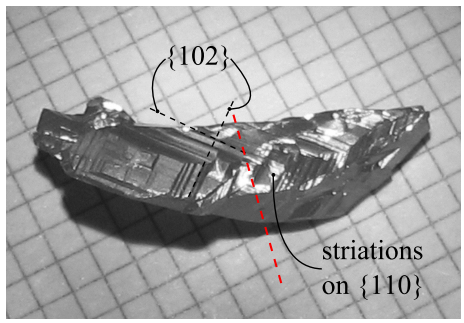

(d)

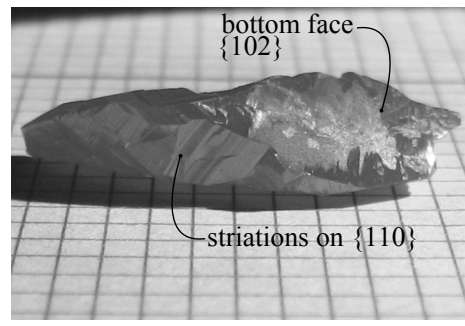

(b)

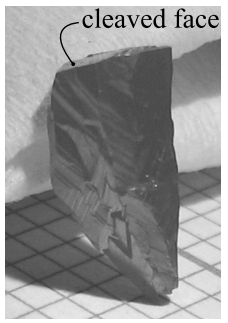

(e)

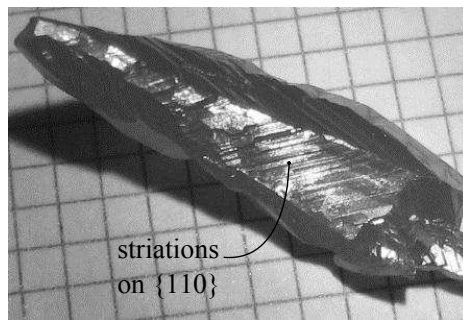

(c)

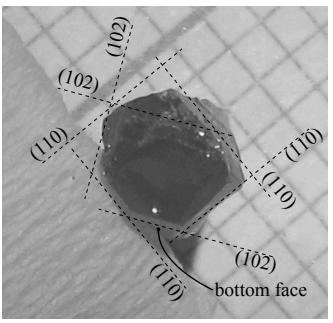

(f)

Figure 5.69. (a)-(d) Observed crystal faces on another twin crystal grown without any additive in a horizontal furnace. The slowest growing faces are the $\{110\}$ faces, identified from the striations. The red dotted line in (d) denotes where the crystal was cleaved (at the (001) cleavage plane. (e) Half of the cleaved crystal. (f) Faces intersecting the (001) plane can be identified and confirm the apparent crytal faces in the morphology.

bottom face ((110) in both Figs. 5.65 and 5.67, (102) in Fig. 5.69).

The addition of low $\overline{\mathrm{M}}_{\mathrm{w}}$ polyethylene to $\mathrm{HgI}_{2}$ growth system appears to make the $\{110\}$ faces less faceted and simultaneously reduce the growth rate of the $\{110\}$ faces closer or even lower than that of the $\{001\}$ faces, as evidenced by $\mathrm{HgI}_{2}$ crystals and their approximate crystal models in Figs. 5.70 and 5.71. Fig. 5.70(a) shows $\mathrm{HgI}_{2}$ single crystals grown with low $\overline{\mathrm{M}}_{\mathrm{w}}$ polyethylene. WinXMorph-created crystal models of several observed shapes are shown in Figs. 5.70(b) to (d), with $r_{\{h k l\}}$ shown in Fig. 5.70. All three crystal models, which approximate several crystals in Fig. 5.70(a), are bounded mainly by the $\{001\}$ and $\{110\}$ crystal forms. Fig. 5.70(b) appears to be a variation of Case $\mathbf{B}$ (or $\mathbf{T}$ ), with $r_{\{110\}}$ being much lower than the rest, whereas Fig. 5.70(c) is a variation of Case F, and Fig. 5.70(d) is a variation of Case $\mathbf{A}$, with higher values of $r_{\{101\}}, r_{\{102\}}$ and $r_{\{103\}}$ (crystal model cases can be found in Table 5.8). Most crystals feature several $(h 0 l)$ faces growing in parts of the crystals facing away from the source, and to form these faces in the crystal models, several values of $r_{(h 0 l)}$ are set lower than the rest of $r_{\{h 0 l\}}$. 
One of the individual crystals in a twin crystal pictured in Fig. 5.71 (also pictured in Fig. 5.57(a)) has a similar shape to the single crystal in Fig. 5.70(a), with a crystal model having a similar ratio of $r_{\{h k l\}}$ values to those of Fig. 5.70(b), along with a few exceptions. During the creation of the model, it was found that the values of $r_{\{101\}}$ ranged between 4.1 and 6 (or equal to $r_{\{102\}}$ and $r_{\{103\}}$ ), if $\{101\}$ faces except (101) were to be excluded from the crystal morphology. This observation reiterates that the presence or absence of a crystal form or face depends on the presence or absence of other crystal forms or faces, which can be determined from the ratio of the central distances of the faces, $r_{\{h k l\}}$. $\mathrm{HgI}_{2}$ crystals grown with 3-hexadecanone (Section 5.6.1) resemble the crystal model in Fig. 5.70(d), while crystals grown with 14-heptacosanone (Section 5.6.2) resemble the crystal model in Fig. 5.70(b).

Summary of the approximate $r_{\{h k l\}}$ values for all crystals in this section can be found in Table 5.9, with the largest $r_{\{h k l\}}$ values listed, whereas smaller individual $r_{(h k l)}$ can be found in the corresponding figures or text. The purpose of the summary is to compare trends in $r_{\{h k l\}}$ values between the overall crystal shapes for crystals grown with different growth methods and in the presence of an additive, namely low $\overline{\mathbf{M}}_{\mathrm{w}}$ polyethylene. In general, $r_{\{001\}}$ $<r_{\{110\}}<r_{\{h 0 l\}}$ for $\mathrm{HgI}_{2}$ single crystals grown without any additive in a vertical furnace, whereas $r_{\{110\}} \leq r_{\{001\}} \leq r_{\{h 0 l\}}$ for $\mathrm{HgI}_{2}$ single crystals grown without any additive in horizontal furnace. For crystals grown with low $\overline{\mathrm{M}}_{\mathrm{w}}$ polyethylene, especially tetragonal prismatic crystals, for example, in Fig. 5.71, $r_{\{110\}} \ll r_{\{001\}}$, and $r_{\{110\}}<r_{\{101\}} \leq r_{\{102\}}$ $\sim r_{\{103\}}$. Although shaped single crystals, such as Fig. 5.70(c), and platelet crystals, such as Fig. 5.70(d), grow less frequently during $\mathrm{HgI}_{2}$ growth with low $\overline{\mathrm{M}}_{\mathrm{w}}$ polyethylene, they are still bounded by the $\{001\}$ and $\{110\}$ crystal forms. 
Table 5.9. Summary of normalized $r_{\{h k l\}}$ values (to the first decimal).

\begin{tabular}{ccccc}
\hline \multicolumn{5}{c}{ No additive } \\
\hline$r_{\{h k l\}}$ & Vertical growth & $\begin{array}{c}\text { Horizontal growth, } \\
\text { single crystal } \\
\text { (Fig. 5.64) }\end{array}$ & $\begin{array}{c}\text { Horizontal growth, } \\
\text { single crystal } \\
\text { (Fig. 5.65) }\end{array}$ & $\begin{array}{c}\text { Horizontal growth, } \\
\text { twin crystal } \\
\text { (Fig. 5.68) }\end{array}$ \\
\hline$r_{\{001\}}$ & 1 & 1 & 1.6 & 2 \\
$r_{\{110\}}$ & 2.3 & 2.4 & 1 & 1 \\
$r_{\{101\}}$ & 2.6 & 3.1 & 2.5 & 2.3 \\
$r_{\{102\}}$ & 2.3 & 2.6 & 1.9 & 2.3 \\
$r_{\{103\}}$ & 2.6 & 2.6 & 2.5 & 2.3 \\
\hline & \multicolumn{5}{c}{ (Fig. 5.63) } & With low $\bar{M}_{\mathrm{w}}$ polyethylene & \\
\hline$r_{\{h k l\}}$ & Single crystal (b) & Single crystal (c) & Single crystal (d) & Twin crystal \\
& (Fig. 5.70) & (Fig. 5.70) & (Fig. 5.70) & (Fig. 5.71) \\
\hline$r_{\{001\}}$ & 4 & 1 & 1 & 4.2 \\
$r_{\{110\}}$ & 1 & 1 & 2 & 1 \\
$r_{\{101\}}$ & 4 & 2.3 & 3 & 4 \\
$r_{\{102\}}$ & 4 & 2.3 & 3 & 4 \\
$r_{\{103\}}$ & 4 & 2.3 & 4 & 4.7 \\
\hline
\end{tabular}




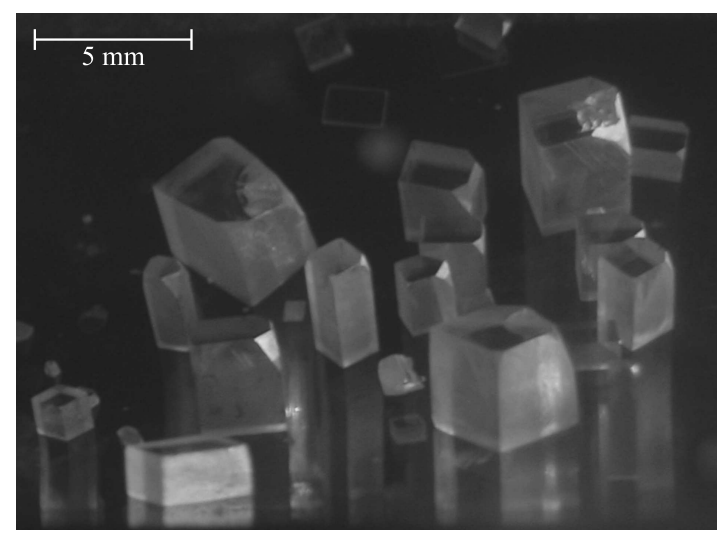

(a)

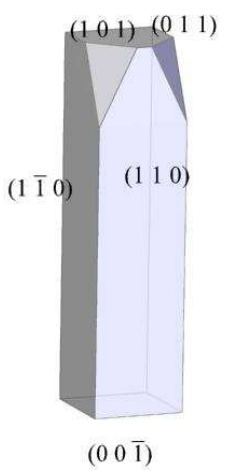

(b)

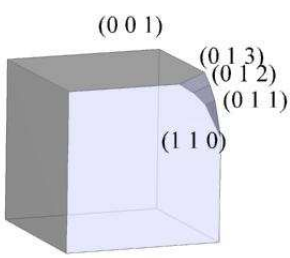

(c)

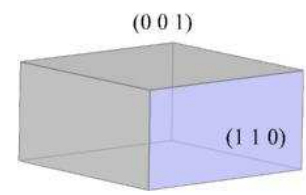

(d)

\begin{tabular}{rlllll}
\hline (b) & $r_{\{001\}}=4$ & $r_{\{110\}}=1$ & $r_{\{101\}}=4$ & $r_{\{102\}}=4$ & $r_{\{103\}}=4$ \\
exceptions: & $r_{(101)}=2.1$ & $r_{(011)}=2.1$ & & & \\
(c) & $r_{\{001\}}=1$ & $r_{\{110\}}=1$ & $r_{\{101\}}=2.3$ & $r_{\{102\}}=2.3$ & $r_{\{103\}}=2.3$ \\
exceptions: & $r_{(011)}=1.4$ & $r_{(012)}=1.4$ & $r_{(013)}=1.4$ & & \\
(d) & $r_{\{001\}}=1$ & $r_{\{110\}}=2$ & $r_{\{101\}}=3$ & $r_{\{102\}}=3$ & $r_{\{103\}}=4$
\end{tabular}

Figure 5.70. a) Single crystals grown with low $\overline{\mathbf{M}}_{\mathrm{w}}$ polyethylene in a horizontal furnace are approximated by (b)-(d) crystal models created using WinXMorph with listed $r_{\{h k l\}}$ values. 


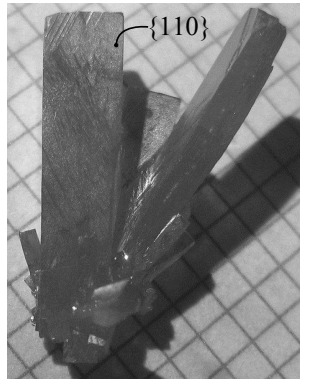

(a)

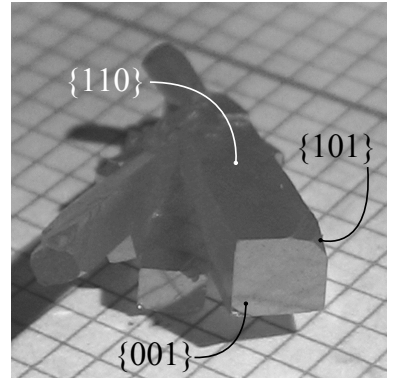

(b)

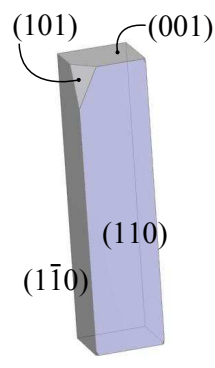

(c)

$$
\text { exceptions: } \quad r_{(101)}=3.5 \quad r_{(\overline{1} \overline{1} 0)}=1
$$

Figure 5.71. (a) A twin crystal grown with low $\overline{\mathrm{M}}_{\mathrm{w}}$ polyethylene in a horizontal furnace, also shown in Fig. (b) An alternate view of the crystal. (c) The crystal model of one of individual twins, created using WinXMorph with $r_{\{h k l\}}$ values listed above. 


\subsubsection{Proposed hypothesis}

As mentioned in Theory (Section 3), at room temperature $\alpha-\mathrm{HgI}_{2}$ exists in a tetragonal crystal structure, where $\mathrm{HgI}_{4}$ tetrahedra layers held by van der Waals forces are stacked along the [001] direction ( $c$-axis). $\mathrm{HgI}_{2}$ exists as a linear molecule in vapor within the temperature and pressure ranges used during growth, where the Hg-I bond is covalent. Therefore, it is deemed reasonable to assume that $\mathrm{HgI}_{2}$ molecules, not separate $\mathrm{Hg}$ and $\mathrm{I}$ atoms, are the crystal building blocks. Because the growth of the $\{110\}$ faces seems to be the most affected orientation by the organic additives, the atomic make-up of these faces are examined in this section.

Fig. 5.72(a) shows one $\mathrm{HgI}_{4}$ tetrahedron (for comparison see Fig. 3.3(a) in Theory) in

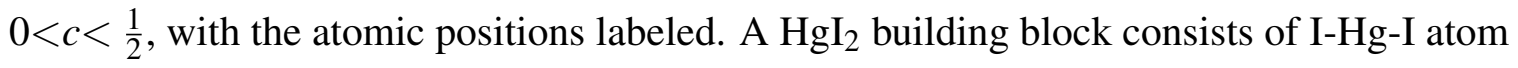
pairs labeled either $\mathrm{I}_{1}-\mathrm{Hg}-\mathrm{I}_{2}, \mathrm{I}_{1}-\mathrm{Hg}-\mathrm{I}_{4}, \mathrm{I}_{3}-\mathrm{Hg}-\mathrm{I}_{2}$, or $\mathrm{I}_{3}-\mathrm{Hg}-\mathrm{I}_{4}$, but for this analysis $\mathrm{I}_{1}-\mathrm{Hg}-\mathrm{I}_{2}$ is used to define a $\mathrm{HgI}_{2}$ building block. Two additional building blocks are added, each along either the [110] or [010] direction, such that iodine atoms $I_{3}$ and $I_{4}$ are incorporated to complete the four corners in the tetrahedron (Fig. 5.72(b). The solid lines indicate covalent bonds between mercury and iodine atoms within the same building block, while the dotted lines indicate covalent bonds between mercury and iodine atoms in adjacent building blocks. In reality there is no such difference, but this distinction is needed for analysis purposes.

Another layer of building blocks are added, completing the tetrahedra in the process, resulting in the growth of the (001) plane in the [1110] direction (Fig. 5.72(c)). To further expand the (001) plane, building blocks can also be added in the [110] direction (Fig. 5.72(d)) or other $\langle 110\rangle$ directions, resulting in the growth of (001) plane in the $\langle 110\rangle$ directions (Fig. 5.72(e)), where mercury layer is the top-most layer in the (1110) face and iodine layers are the top-most layers in (110), (110), and (( $\overline{1} \overline{1} 0)$ faces. The same explanations and derivations can be applied to another $\mathrm{HgI}_{4}$ tetrahedron in $\frac{1}{2}<c<1$ (Fig. 5.73(a)), where $\mathrm{I}_{A}-\mathrm{Hg}-\mathrm{I}_{B}$ is the building block in the pictured unit cell (Figs. 5.73(b)-(e)). In this case, a mercury layer is the top-most layer in the (110) face and iodine layers are the top-most layers in 


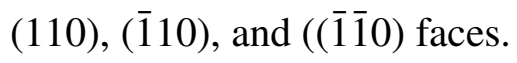

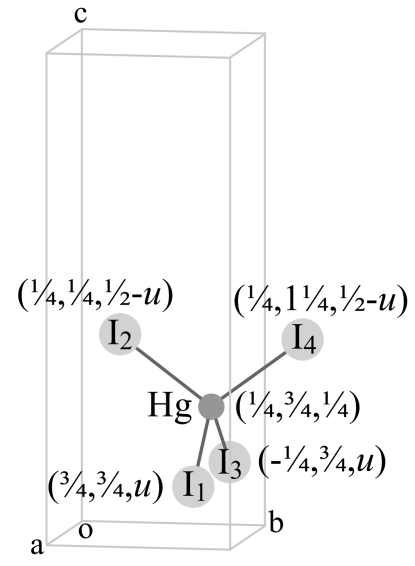

(a)

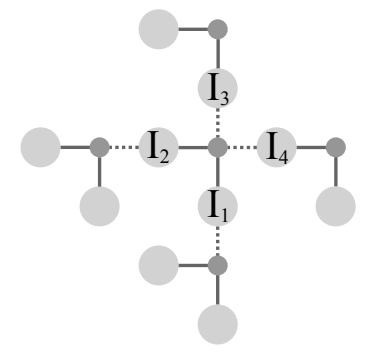

(d)

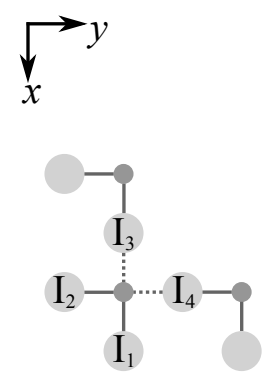

(b)

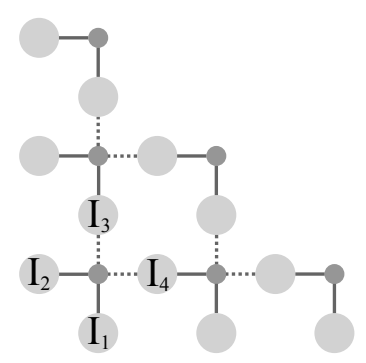

(c)

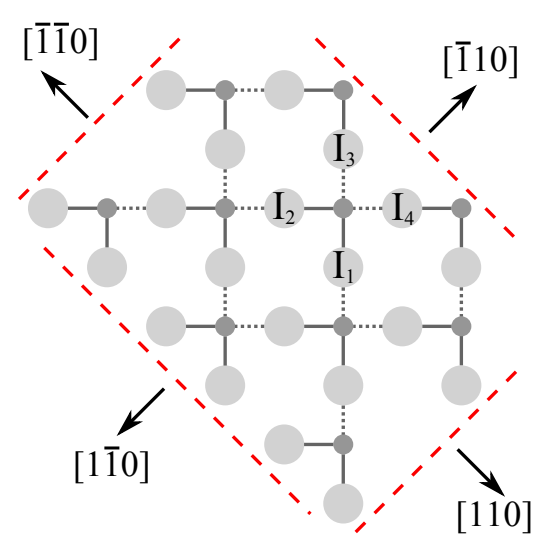

(e)

Figure 5.72. (a) $\mathrm{A} \mathrm{HgI}_{4}$ tetrahedron within $0<c<\frac{1}{2}$, where $\mathrm{I}_{1}-\mathrm{Hg}-\mathrm{I}_{2}$ is the building block in the pictured unit cell. (b) The tetrahedron is constructed by adding two more building blocks to $I_{1}-H g-I_{2}$, such that $I_{3}$ and $I_{4}$ form the other two corners in the tetrahedron. (c) Addition of another layer of building blocks, which completes the tetrahedra in the process, expands the (001) plane in the [110] direction. (d) Building blocks can also be added to expand the (001) plane in the [11 10$]$ direction. (e) Overall, adding building blocks expands the (001) layer in the $\langle 110\rangle$ directions. 


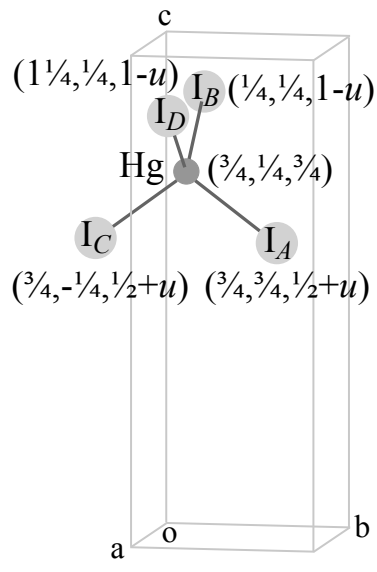

(a)

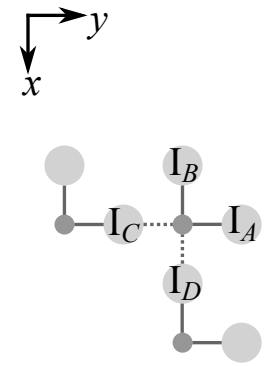

(b)

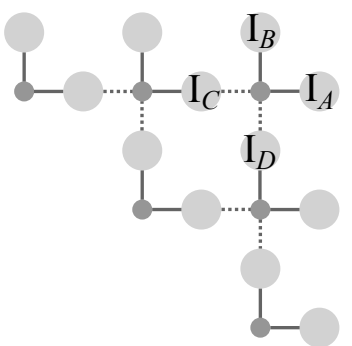

(c)

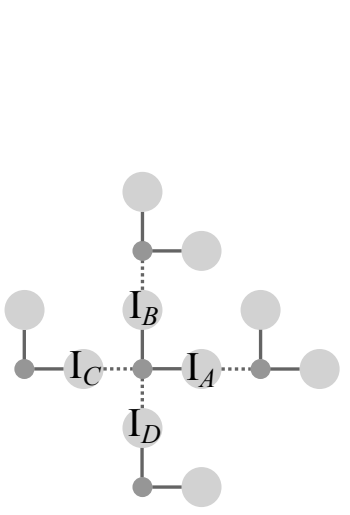

(d)

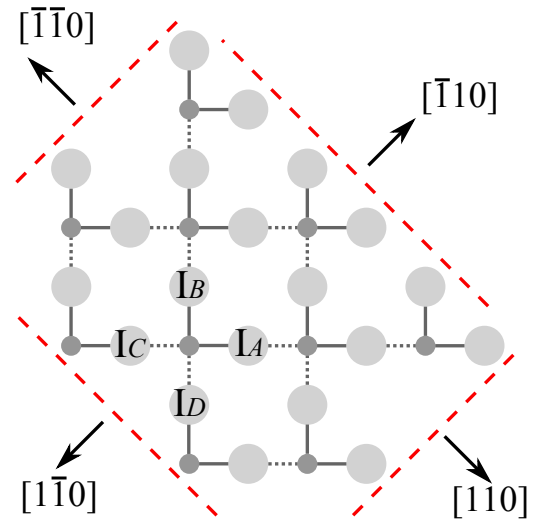

(e)

Figure 5.73. (a) $\mathrm{A} \mathrm{HgI}_{4}$ tetrahedron within $\frac{1}{2}<c<1$, where $\mathrm{I}_{A}-\mathrm{Hg}-\mathrm{I}_{B}$ is the building block in the pictured unit cell. (b) The tetrahedron is constructed by adding two more building blocks to $\mathrm{I}_{A}-\mathrm{Hg}-\mathrm{I}_{B}$, such that $\mathrm{I}_{C}$ and $\mathrm{I}_{D}$ form the other two corners in the tetrahedron. (c) Addition of another layer of building blocks, which completes the tetrahedra, expands the (001) plane in the [110] direction. (d) Building blocks can also be added to expand the (001) plane in the [110] direction. (e) As in the case in Fig. 5.72(e), adding building blocks expands the (001) layer in the $\langle 110\rangle$ directions. 
When the (001) plane-projected tetrahedral layers in $0<c<\frac{1}{2}$ (Fig. 5.72(e)) and $\frac{1}{2}<c<1$ (Fig. 5.73(e)) are observed simultaneously, the top-most layers in the (110) and (110) planes are either mercury or iodine layers, while the top-most layers in the (110) and (1̄10) planes are always iodine layers. Notice that the crystals in Figs. 5.72 and 5.73 are constructed with the assumption that $\mathrm{HgI}_{2}$ are incorporated into the crystals as complete molecules, and thus, the crystals are stoichiometric. According to [37], however, creation of $\mathrm{HgI}_{4}$ tetrahedra demands breakage and creation of (covalent) bonds, which means that at a point in time, as one $\mathrm{HgI}_{2}$ linear molecule is about to be incorporated into the crystal lattice, its $\mathrm{Hg}$-I bonds may break. As a result, $\mathrm{HgI}^{+}$and $\mathrm{I}^{-}$, or even $\mathrm{Hg}^{2+}$ and two $\mathrm{I}^{-}$, can also be incorporated into the crystal lattice with the stoichiometry intact.

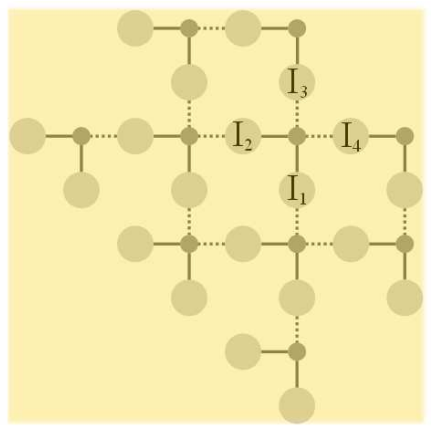

(a) (001) in $0<c<\frac{1}{2}$

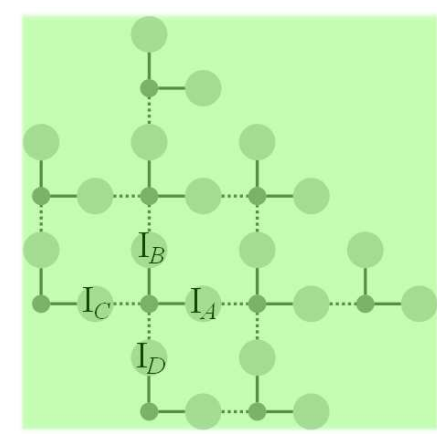

(b) (001) in $\frac{1}{2}<c<1$

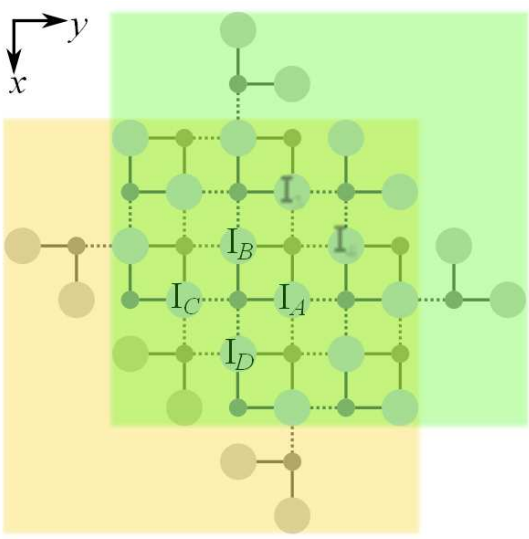

(c) Combination of (a) and (b)

Figure 5.74. Two tetrahedral layers in $0<c<1$, as projected onto the (001) plane. (a) The layer in $0<c<\frac{1}{2}$ is highlighted in yellow, while (b) the layer in $\frac{1}{2}<c<1$ is highlighted in green, for ease of identification when (c) the two layers are observed simultaneously. Iodine atom $\mathrm{I}_{A}$ is directly above $\mathrm{I}_{1}$, while $\mathrm{I}_{B}$ is directly above $\mathrm{I}_{2}$.

Examples of $\mathrm{HgI}^{+}$and $\mathrm{I}^{-}$or $\mathrm{Hg}^{2+}$ and two $\mathrm{I}^{-}$incorporation into the (001) plane, and the resulting effects on the atomic make-up of the top-most layers in the $\langle 110\rangle \mathrm{di}$ rections, are shown in Fig. 5.75. Addition of $\mathrm{HgI}^{+}$and $\mathrm{I}^{-}$(both ions in thicker outlines) to Fig. 5.72(e) results in either Figs. 5.75(a) or 5.75(b), whereas addition of $\mathrm{Hg}^{2+}$ and two $\mathrm{I}^{-}$(both ions also in thicker outlines) results in Fig. 5.75(c). The consequence of incorporation of ions and/or atoms, instead of whole molecules, into the crystal is the top-most layers in the $\langle 110\rangle$ can be either mercury or iodine layers. 


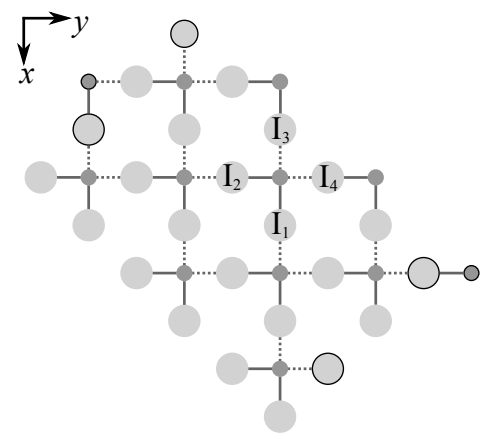

(a)

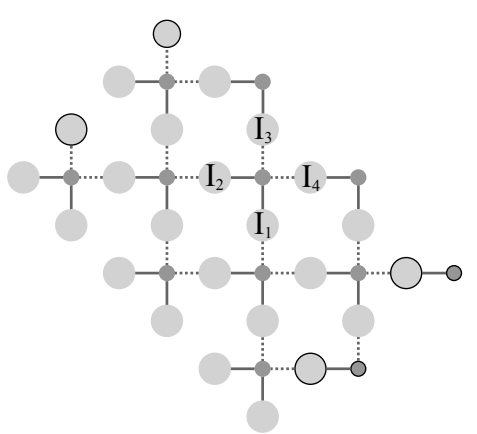

(b)

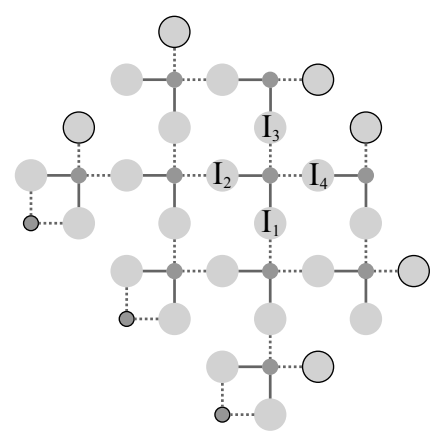

(c)

Figure 5.75. Examples of the addition of $\mathrm{HgI}^{+}$and $\mathrm{I}^{-}$or $\mathrm{Hg}^{2+}$ and two $\mathrm{I}^{-}$ions into the crystal lattice pictured in Fig. 5.72(e).

Because the difference of the electronegativity values between $\mathrm{Hg}$ and $\mathrm{I}$ is close to 0.7 $\left(\mathrm{EN}_{\mathrm{Hg}}=2.00, \mathrm{EN}_{I}=2.66\right)$, the $\mathrm{Hg}-\mathrm{I}$ bond is considered a polar covalent bond, in which the electron density is situated closer to iodine than to mercury (i.e., the electron density is not shared equally). Therefore, the $\mathrm{Hg}$-I bond has an electric dipole, symbolically defined as a vector arrow pointing from the atom with smaller electronegativity $(\mathrm{Hg}, \delta+)$ to the atom with bigger electronegativity (I, $\delta$-) (Fig. 5.76). The actual dipole moment is not calculated in this study, therefore the vector arrow is not drawn to scale and is used qualitatively to explain concepts.

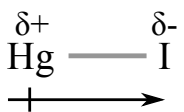

Figure 5.76. $\mathrm{Hg}$-I polar covalent bond and dipole vector.

Qualitative vector analysis of Hg-I dipoles in the (001) plane is shown in Fig. 5.77, based on the tetrahedral layer in $0<c<\frac{1}{2}$ (Fig. 5.72(e)), with a Hg-I dipole vector represented by a blue arrow, and the summation vector in a red arrow. Dipole cancellation occurs within complete $\mathrm{HgI}_{4}$ tetrahedra, whereas non-zero dipole vectors exist in incomplete tetrahedra, which are usually located in the top-most layers in the $\langle 110\rangle$ directions. Dipoles formed with electron-attracting mercury atoms are found in the top-most layer in

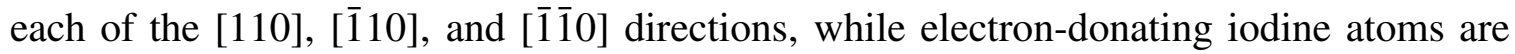
found in the top-most layer in the [1ㅣㅣㄹ direction. Similar vector analysis can be done on 
other configurations of the tetrahedral layer in $0<c<\frac{1}{2}$ (Fig. 5.75).

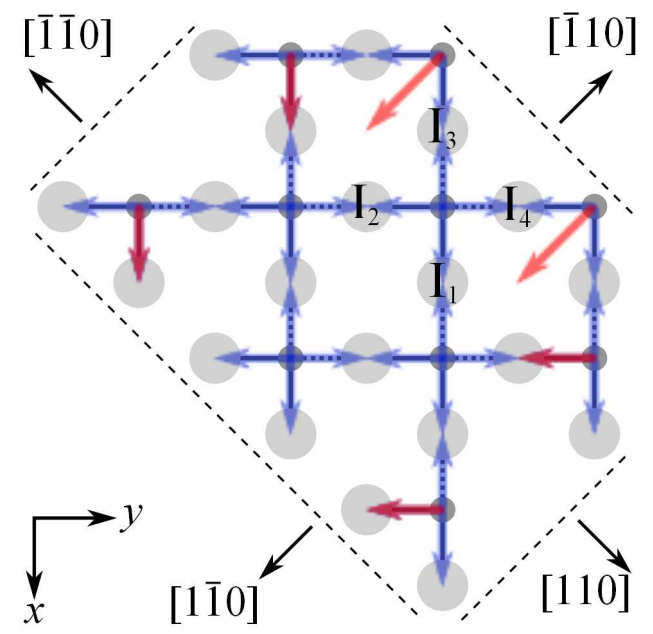

Figure 5.77. Qualitative vector analysis of $\mathrm{Hg}$-I dipoles for a tetrahedral layer in $0<c<\frac{1}{2}$.

The electron-attracting mercury atoms often found in the top-most layer of the $\{110\}$ faces then may attract dipoles, for example, in the form of molecules with polar bonds. Ketones and alkynes, which are volatiles detected in a TD-GC/MS study of low $\overline{\mathrm{M}}_{\mathrm{w}}$ polyethylene (see Section 5.7.1), have C-O double bonds and C-C triple bonds, respectively, both of which are polar bonds. The polar bonds in these molecules (dipoles) may be attracted to the exposed mercury atoms on the top layers of the $\{110\}$ faces through dipole-dipole interaction, and as a result, incoming $\mathrm{HgI}_{2}$ molecules are not able to form covalent bonds at these positions. Therefore, the growth of crystal in the $\langle 110\rangle$ directions are impeded until the foreign molecules are removed. Because the strength of a dipole-dipole interaction is weaker compared to the strength of a covalent bond, the growth rates in the $\langle 110\rangle$ directions, $\Delta r_{\{110\}}$, are decreased, but not terminated, resulting in smaller values of $r_{\{110\}}$. Crystal models in Table 5.8 show that when the ratio of $r_{\{110\}}$ to the rest of $r_{\{h k l\}}$ decreases (compare, for example, Cases A with B), the size of the $\{110\}$ faces increases.

Qualitative vector analysis of $\mathrm{Hg}$-I dipoles in a tetrahedral layer in $\frac{1}{2}<c<1$ is also shown in Fig. 5.78, where a Hg-I dipole vector is represented by a blue arrow, and the summation vector in a green arrow. When the two tetrahedral layers in $0<c<1$ are observed simultaneously, with $\mathrm{I}_{A}$ directly above $\mathrm{I}_{1}$ and $\mathrm{I}_{B}$ above $\mathrm{I}_{2}$, the mercury atoms in the incom- 


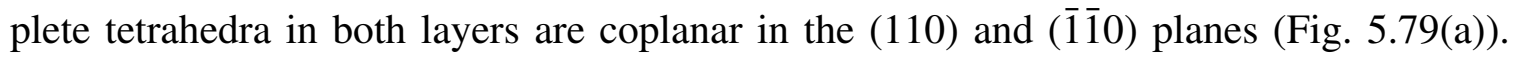
These mercury atoms are pairs of mercury atoms in unit cells along the [1110] or [1110] directions, where within a $\mathrm{HgI}_{2}$ unit cell, the mercury ions lie in one of $\langle 111\rangle$ directions. For example, in the unit cell shown in Fig. 5.79(b), the mercury atoms lie along [1̄111], which is coplanar in both (101) and (110) according to the analysis done in Section 5.9.2. This analysis implies that electron-attracting mercury atoms are also found in the top-most layer of a $\{101\}$ face, and thus, the growth of the $\{101\}$ faces can be impeded by the attraction of foreign molecules or impurities with polar bonds to the mercury atoms. Crystal models in Table 5.8 show that when the ratio of $r_{\{101\}}$ to the rest of $r_{\{h k l\}}$ decreases (compare, for example, Cases $\mathbf{B}$ with $\mathbf{U})$, the size of the $\{101\}$ faces increase.

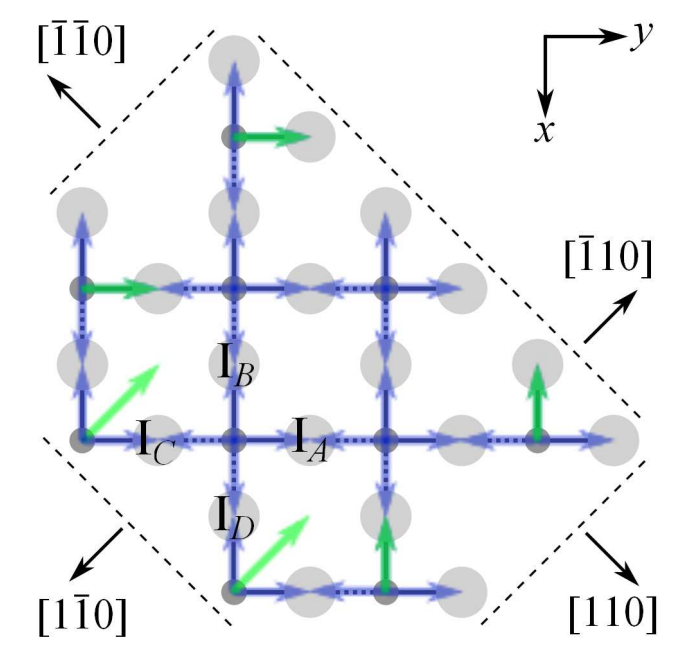

Figure 5.78. Qualitative vector analysis of $\mathrm{Hg}$-I dipoles for a tetrahedral layer in $\frac{1}{2}<c<1$.

One tetrahedral layer consists of one mercury layer sandwiched between two iodine layers, hence, the top-most layer of a (001) face is always an iodine layer. When electrondonating iodine atoms in $\mathrm{Hg}$-I dipoles are in the top-most layers of the $\{001\}$ and $\{110\}$ faces, the growth of these faces can also be impeded when molecules, with polar bonds, are attracted to the iodine atoms through dipole-dipole interactions. Also found in the TD-GC/MS study were alkanes, which have $\mathrm{C}-\mathrm{H}$ bonds that are polar but there is dipole cancellation within the molecule due to symmetry. Therefore, alkanes are not expected to affect the growth of either the $\{001\}$ or $\{110\}$ faces, which appears to be supported by the 


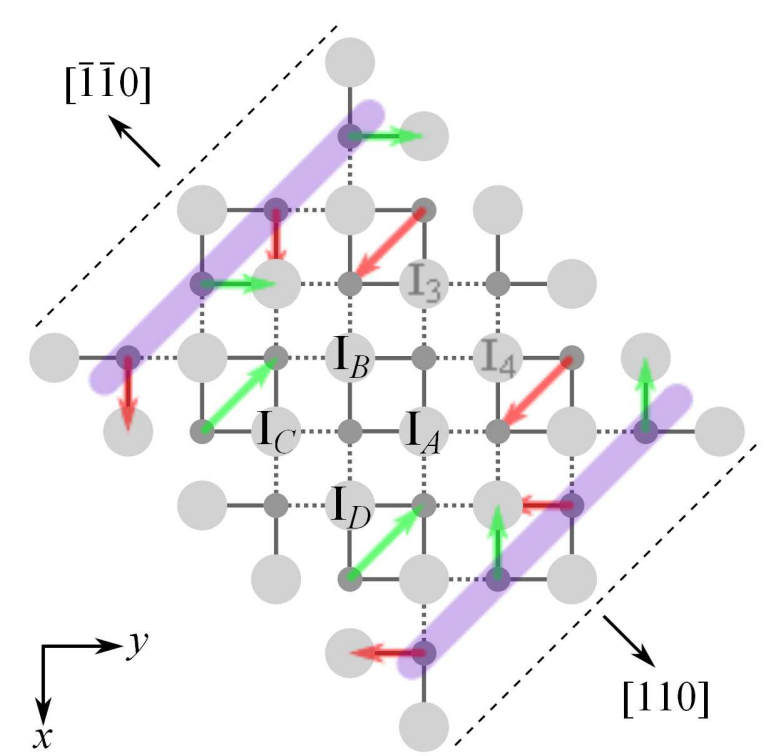

(a)

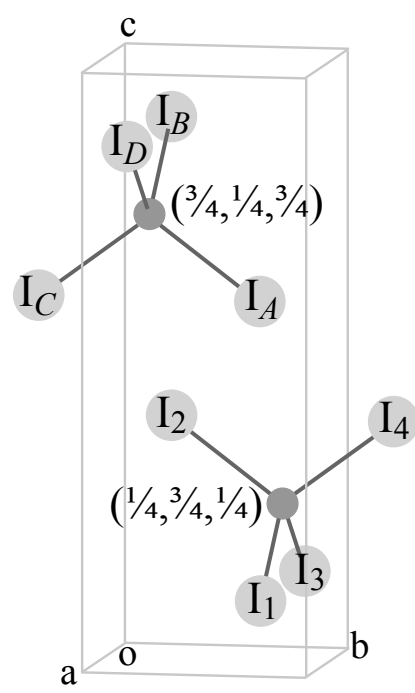

(b)

Figure 5.79. (a) When the two tetrahedral layers in $0<c<1$ are observed simultaneously, the mercury atoms in the incomplete tetrahedra in both layers are coplanar in the (110) and (1̄10) planes. (b) The tetrahedral layers are lined up with $\mathrm{I}_{A}$ right above $\mathrm{I}_{1}$ and $\mathrm{I}_{B}$ above $\mathrm{I}_{2}$.

experimental data. 


\section{Chapter 6}

\section{Conclusions}

The results of the TD-GC/MS studies on the low molecular weight polyethylene and the growth of $\mathrm{HgI}_{2}$ tetragonal prismatic crystals, with either the low molecular weight polyethylene or ketone (Section 5.7.1), indicated that the morphology of $\mathrm{HgI}_{2}$ crystals was not affected by the polyethylene itself, but rather, by polar-bond-containing impurities within the starting polyethylene. Electron-attracting mercury atoms, often found in the top-most layer of both the $\{110\}$ and $\{101\}$ faces (Figs. 5.77 and 5.78) may attract these impurities through dipole-dipole interaction, and consequently, incoming $\mathrm{HgI}_{2}$ molecules are not able to form covalent bonds at these positions. Therefore, the growth of crystal in the $\langle 110\rangle$ and $\langle 101\rangle$ directions are impeded until the foreign molecules are removed (Section 5.9.5).

The BFDH crystal models, created for $\mathrm{HgI}_{2}$ crystals grown with low molecular weight polyethylene, show that when the ratio of $r_{\{110\}}$ to the rest of $r_{\{h k l\}}$ decreases, the size of the $\{110\}$ faces increases (compare, for example, Cases $\mathbf{A}$ to $\mathbf{B}$, in Table 5.8). The presence of striations indicative of alternating growth between the $\{110\}$ and $\{101\}$ faces also suggests that the growth rate of the $\{101\}$ might also be lower than the $\{001\}$ and other $\{h 0 l\}$ faces, but still larger than that of the $\{110\}$ faces. The overall affect on the $\mathrm{HgI}_{2}$ crystal morphology is the elongation of the crystal in the $\{001\}$ directions, and the bounding of the crystal primarily with the $\{110\}$ faces, therefore, the crystal assumes a 
tetragonal prismatic shape.

Explanations on the effect of polyethylene addition to $\mathrm{HgI}_{2}$ crystallization, posed by previous studies [28-30, 47-52], have been proven inadequate. Even though the use of non-purified $\mathrm{HgI}_{2}$ was suggested [28, 47], the contents of the growth ampoule cannot be ascertained accurately. While vastly different crystal morphologies might be obtained, depending on the purity of the starting $\mathrm{HgI}_{2}[28,29,47]$, yet there is a large uncertainty in this growth procedure, which deems it unrepeatable and impractical.

There also exist many types of polyethylene that differ in production methods, densities, molecular weights, and fillers or impurities, hence, care must be taken to mention the characteristics of the polyethylene employed in the process. As shown in Section 5.3, types of polyethylene utilized do affect $\mathrm{HgI}_{2}$ crystallization and crystal morphology. In particular, the presence and the types of organic volatiles produced from each type of polyethylene seems to be the biggest influence in $\mathrm{HgI}_{2}$ growth in the horizontal furnace. From the TD-GC/MS results (Section 5.7.1), the ultra high $\overline{\mathrm{M}}_{\mathrm{w}}$ polyethylene produced little to no volatiles upon being heated (below the detection limit of the instrument). Mostly twin crystals with prismatic shapes but with no pronounced orientation along any particular crystallographic axis, were obtained. When the low $\overline{\mathrm{M}}_{\mathrm{w}}$ polyethylene was utilized, various alkanes and ketones were produced, and crystals elongated in the $c$-axis were acquired.

The presence of organic volatiles may indeed increase the total pressure in the growth ampoule, and may influence the mass transport of $\mathrm{HgI}_{2}$. However, the mass transport is not the only factor that affects the change in $\mathrm{HgI}_{2}$ crystal morphology. Shown in the growth with residual pressure (Section 5.2.2), that $\mathrm{HgI}_{2}$ crystals were smaller than the ones grown under vacuum, but they had the same crystal morphology. As deduced in Chapter 5, the addition of $1 \mathrm{wt}-\%$ of any of the alkanes (Section 5.5) did not produce as much morphology change to $\mathrm{HgI}_{2}$ as the addition of only $0.1 \mathrm{wt}-\%$ of either of the ketones (Section 5.6). Furthermore, only growth runs with ketones produced $\mathrm{HgI}_{2}$ crystals with shapes similar to those produced with low $\overline{\mathrm{M}}_{\mathrm{w}}$ polyethylene.

Therefore, the statement that polyethylene aids in the vapor transport, as claimed in [29], is inconclusive. 
Burger 1982 - Because it was assumed that polyethylene disassociated to molecules with $\overline{\mathrm{M}}_{\mathrm{w}}$ about 700 , the interpretation was that polyethylene did not aid in physical vapor transport, but rather aided chemically in vapor transport. The explanation on the aiding process of the polyethylene in the chemical transport, however, was not given.

Burger 1985 - Thus, the study concluded that polyethylene played a role in purifying the starting material by separating impurities (for example, $\mathrm{Hg}_{2} \mathrm{I}_{2}$ ) from $\mathrm{HgI}_{2}$.

Laskowski et al - The study also noticed that some of the organics decomposed during the growth, whether due to high temperature or mixing with $\mathrm{HgI}_{2}$, causing a variation in partial and total pressures were expected, as indicated by an increase in pressure attributed to the residual gases $[31,41]$. The effects of the stoichiometry and purity of the starting $\mathrm{HgI}_{2}$ were deemed inconsequential, unlike claimed previously in [29, 30]

Gases - Therefore, the organic materials seemed to behave as inert gases, prompting futher studies in $\mathrm{HgI}_{2}$ mass transport in horizontal furnaces [48-51], as well as $\mathrm{HgI}_{2}$ with inert gases $[52,53]$

Faile - Purification of the starting $\mathrm{HgI}_{2}$ was not done because the author believed that $\mathrm{HgI}_{2}$ purification was not needed to produce platelet crystals. Although the formation of $\mathrm{HgI}_{2}$ platelet was not known, and was not attempted to be proven, the author assumed that polymer transport, formation, and/or disassociation might be the factors that influenced the platelet formation.

\subsection{Future works}

Several studies on the properties of $\mathrm{HgI}_{2}$ tetragonal prismatic crystals grown in this project are described in Sections 6.1.1.1 and 6.1.1.2. As mentioned in Tables 4.4 and 4.5 in Chapter 4, the alkanes and ketones utilized in the growth experiments were not purified. The consequences of using these unpurified organics are described in Section 6.1.2. The $\mathrm{BFDH}$ rules were used to create $\mathrm{HgI}_{2}$ crystal models (Section 3), which were then used as comparisons to crystal shapes. The BFDH rules, however, were derived without including energy consideration, and they depend only on crystal geometry and symmetry (Section 
3.2.1). Other possible crystal models that include the energy consideration are described in Section 6.1.3.

\subsubsection{Examinations on crystal properties}

The integrity of the $\mathrm{HgI}_{2}$ single crytals can be examined using the X-ray diffraction by the Laue method (Section 6.1.1.1). The bandgap of the red crystals and trap levels can be ascertained by photoluminescence (Section 6.1.1.2).

\subsubsection{X-Ray diffraction by Laue back-reflection method}

X-ray diffraction can be explained the formula derived by Bragg (based on the formula derived by von Laue). Consider a parallel beam of monoenergetic x-rays that are incident on a crystal. Assuming that, for a specific set of crystallographic planes, the incident angle (called Bragg angle $\theta$ ) is equal to the reflection angle. Constructive interference from rays reflected from two consecutive planes, with interplanar distance $d$, occurs when the difference in path travelled by the $\mathrm{x}$-rays, $2 d \sin \theta$, is equal to the integral multiple $n$ of the incident wavelength $\lambda$, or

$$
n \lambda=2 d \sin \theta
$$

The above equation is also called the Bragg condition. The resulting strong reflection from each set of planes is observed as an intense scattered peak called the Bragg peak, which is captured as a dot in a receiving film or screen positioned to capture the reflected rays.

Crystal examination by X-ray diffraction can be accomplished by either keeping either $\lambda$ or $\theta$ fixed, while varying the other. For a fixed $\lambda, \theta$ can be altered by either varying the direction of the incident x-rays or varying the orientation of the crystal. For a single crystal, only certain $d$ and $\theta$ combinations fulfill the Bragg condition, hence distinct Bragg peaks can be observed. When the sample is polycrystalline, however, numerous $d$ and $\theta$ combinations exist, thus, diffraction rings are obtained, instead of distinct diffraction peaks. 
The same observations can be expected when the Laue method is utilized. This method is employs an X-ray source with multiple wavelengths (i.e., as if $\lambda$ is varied), and a fixed incident beam direction or a fixed sample position (i.e., a fixed $\theta$ ). There are two modes in the Laue method, which are the transmission mode, where forward-scattered beam may traverse the crystal before being recorded, and the back-reflection mode, where back-scattered beam are recorded. The back-reflection method allows for examination of bulk crystals, whereas thin samples must be preparred for crystal examination with the transmission mode to avoid self-absorbtion of the scattered rays. Moreover, because $\mathrm{HgI}_{2}$ has high attenuation probability for X-rays of low energy (Fig. 1.1), the back-reflection method is preferred. Detailed descriptions on both X-ray diffraction and Laue back-reflection method can be found in $[72,73]$.

\subsubsection{Material analysis with photoluminescence}

Photoluminescence refers to light emission from electronic excitation (within atoms, molecules, or ions) that are caused by absorption of light from an external source. This phenomenon is used as a non-destructive analysis method to characterize the electronic states of the material, from which information such as the bandgap energy and the trap levels can be ascertained. Photoluminescence spectroscopy has been used to analyzed bulk $\mathrm{HgI}_{2}$ bandgap energy [74-76] and material quality [77, 78], also in terms of stoichiometry and trap levels [79-81]. Therefore, this method can also be used to determine the bandgap energy the tetragonal prismatic $\mathrm{HgI}_{2}$ crystals as a means to confirm that the crystals are indeed $\alpha-\mathrm{HgI}_{2}$.

\subsubsection{Purity of the starting organic materials}

The TD-GC/MS results (Section 5.7.1) reveal that ketones and alkanes were produced when the low $\overline{\mathrm{M}}_{\mathrm{w}}$ polyethylene was heated in the absence of $\mathrm{HgI}_{2}$ at the temperatures for the pre-growth polyethylene processing (Section 4.5.1). The investigation on the origin of these compounds or how these compounds formed was not pursued in this study, however, 
one possible reason may be linked to the purity of the starting organics. The polyethylene powders, as well as the alkanes and ketones were not purified, with purity levels for the alkanes and ketones are shown in Tables 4.4 and 4.5, respectively, in Chapter 4. Moreover, these organic materials were stored in air, making surface oxidation or formation of $\mathrm{C}-\mathrm{O}$ bonds possible.

Therefore, purification of the polyethylene, alkanes and ketones (or obtaining purer organic materials), and subsequent $\mathrm{HgI}_{2}$ with these purified compounds, must be pursued for further confirmation of the proposed hypothesis (Section 5.9.5). However, the fact that tetragonal prismatic crystals were obtained with 0.1 wt- $\%$ ketone (Table 4.6 and Section

5.6), as compared to 1 - $2 \%$ polyethylene or alkane, does exhibit that ketone significantly affects $\mathrm{HgI}_{2}$ morphology, more so than polyethylene or alkane. Growth of other crystals, especially the ones with non-cubic structures, may benefit from the application of additives that can form non-permanent bonds (e.g., dipole-dipole bonds) to certain crystallographic planes or faces, for the purpose of altering crystal morphology, or encouraging growth in a specific crystallographic direction.

\subsubsection{Energy consideration for crystal models}

As mentioned in Section 3.2.1, the BFDH crytal models consider only the crytal geometry and symmetry, while disregarding any energy consideration (e.g. surface energy or otherwise). Crystal models formed from crystal morphology formulations that include energy consideration, such as the periodic bond chain (PBC, also known as the HartmanPerdok (HP) theory), may be able to explicitly describe the dipole-dipole bonds between the polar bonds in the organic materials and the $\mathrm{Hg}$-I bonds. Therefore, a (semi-)quantitative analysis on the effect may be derived. 


\section{Appendix A}

\section{Factorial Analysis}

\section{A.1 $2^{k}$ factorial design}

Factorial analysis is an experimental design suitable for experiments that consider many factors. Among a number of factorial designs, the $2^{k}$ full factorial is one of the most commonly used. In the $2^{k}$ factorial, two levels labeled high (plus sign + ) and low (minus sign -), respectively, are assigned to each of $\mathrm{k}$ number of factors. The total number of experiments required to complete the design is $2^{k}$ (hence the name). The level of each factor is varied accordingly for each experiment, as shown in the first three columns of Table A.1 for hypothetical factors $\mathbf{A}, \mathbf{B}$, and $\mathbf{C}$.

Specific experimental results on which the effects of the factors are to be determined are called observables (column $\mathbf{W}$ in Table A.1). The observables are analyzed by using an algorithm developed by Yates [82]. To use this algorithm, the observables must be placed in standard order. This order means that the first column has minus and plus sign pairs in a row, the second has two minus and two plus sign pairs in a row, the third has four minus and four plus sign pairs in a row. Table A.1 shows an example for the case of three factors. The observables $\mathbf{W}$ can now be grouped as pairs (for example $\mathrm{W}_{5}$ and $\mathrm{W}_{6}$ are in a pair).

For general $2^{k}$ designs, $\mathrm{k}$ columns of calculations are generated. In Table A.1, columns $\mathrm{X}, \mathrm{Y}$, and $\mathrm{Z}$ show the calculations for a $2^{3}$ design. Each of the first $\frac{k}{2}$ entries in each 
Table A.1. Yates' algorithm for $2^{3}$ full factorial design.

\begin{tabular}{|c|c|c|c|c|c|c|c|c|c|}
\hline No. & $\mathbf{A}$ & $\mathbf{B}$ & $\mathbf{C}$ & $\mathbf{W}$ & $\mathbf{X}$ & $\mathbf{Y}$ & $\mathbf{Z}$ & Effect & ID \\
\hline 1 & - & - & - & $\mathrm{W}_{1}$ & $\mathrm{X}_{1}=\mathrm{W}_{1}+\mathrm{W}_{2}$ & $\mathrm{Y}_{1}=\mathrm{X}_{1}+\mathrm{X}_{2}$ & $\mathrm{Z}_{1}=\mathrm{Y}_{1}+\mathrm{Y}_{2}$ & $\frac{Z_{1}}{8}$ & $(1)$ \\
\hline 2 & + & - & - & $\mathrm{W}_{2}$ & $\mathrm{X}_{2}=\mathrm{W}_{3}+\mathrm{W}_{4}$ & $Y_{2}=X_{3}+X_{4}$ & $\mathrm{Z}_{2}=\mathrm{Y}_{3}+\mathrm{Y}_{4}$ & $\frac{Z_{2}}{4}$ & $A$ \\
\hline 3 & - & + & - & $\mathrm{W}_{3}$ & $\mathrm{X}_{3}=\mathrm{W}_{5}+\mathrm{W}_{6}$ & $Y_{3}=X_{5}+X_{6}$ & $Z_{3}=Y_{5}+Y_{6}$ & $\frac{Z_{3}}{4}$ & $B$ \\
\hline 4 & + & + & - & $\mathrm{W}_{4}$ & $\mathrm{X}_{4}=\mathrm{W}_{7}+\mathrm{W}_{8}$ & $\mathrm{Y}_{4}=\mathrm{X}_{7}+\mathrm{X}_{8}$ & $\mathrm{Z}_{4}=\mathrm{Y}_{7}+\mathrm{Y}_{8}$ & $\frac{Z_{4}}{4}$ & $A B$ \\
\hline 5 & - & - & + & $\mathrm{W}_{5}$ & $\mathrm{X}_{5}=\mathrm{W}_{2}-\mathrm{W}_{1}$ & $Y_{5}=X_{2}-X_{1}$ & $\mathrm{Z}_{5}=\mathrm{Y}_{2}-\mathrm{Y}_{1}$ & $\frac{Z_{5}}{4}$ & $C$ \\
\hline 6 & + & - & + & $\mathrm{W}_{6}$ & $\mathrm{X}_{6}=\mathrm{W}_{4}-\mathrm{W}_{3}$ & $Y_{6}=X_{4}-X_{3}$ & $Z_{6}=Y_{4}-Y_{3}$ & $\frac{Z_{6}}{4}$ & $A C$ \\
\hline 7 & - & + & + & $\mathrm{W}_{7}$ & $\mathrm{X}_{7}=\mathrm{W}_{6}-\mathrm{W}_{5}$ & $Y_{7}=X_{6}-X_{5}$ & $\mathrm{Z}_{7}=\mathrm{Y}_{6}-\mathrm{Y}_{5}$ & $\frac{Z_{7}}{4}$ & $B C$ \\
\hline 8 & + & + & + & $\mathrm{W}_{8}$ & $\mathrm{X}_{8}=\mathrm{W}_{8}-\mathrm{W}_{7}$ & $\mathrm{Y}_{8}=\mathrm{X}_{8}-\mathrm{X}_{7}$ & $\mathrm{Z}_{8}=\mathrm{Y}_{8}-\mathrm{Y}_{7}$ & $\frac{Z_{8}}{4}$ & $A B C$ \\
\hline
\end{tabular}

calculation column is the sum of the first and the second numbers in a pair in the previous column (for example $\mathrm{X}_{3}=\mathrm{W}_{5}+\mathrm{W}_{6}$ ). Each of the last $\frac{k}{2}$ entries in each calculation column is the difference of the first number from the second number in a pair in the previous column (for example $\mathrm{X}_{7}=\mathrm{W}_{6}-\mathrm{W}_{5}$ ).

The entries of the last calculation column $\mathrm{Z}$ are called the contrasts. The estimated effect of the factor combination in an experiment is equal to the corresponding contrast divided by $\frac{k}{2}$ with the exception of the first effect. (The first effect is basically the average of all the observable values). The last column ID denotes which one or more factors are at high levels.

\section{A.2 Methods of analysis}

\section{A.2.1 Normal probability plot}

There are several ways to analyze the effects of the observables in a factorial designed experiment. The most common method is to plot the effects in a normal probability plot. In order to construct the normal probability plot, the effects are ordered from the smallest to the largest. The probability p of each effect is calculated: 


$$
p_{i}=\frac{i-0.5}{k}
$$

where $i=1,2, . ., k$. The normal probability plot is constructed by plotting the estimated effects vs. $z$-scores (that can be looked up in a $z$-score table). Effects that are caused by statistical fluctuations fall about a straight line. Those effects that are not statistical in nature (i.e., active effects) fall at locations away from the straight line.

The determination of the normality of a set of data is usually subjective. The rule of thumb is to include data from the $25 \%$ to $75 \%$ percentile or to ignore data points at the end. Quantitative determination of normality has also been developed. One of the methods is a graphical method developed by Mage [83]. This graphical method is based on the normality test based on the Kolmogorov-Smirnov (K-S) statistics [83, 84]. Using the K-S statistics on a sample set of $\mathrm{n}$ data, the value $D_{n}$ is defined as

$$
D_{n}=\max _{X}\left|F_{n}(X)-F_{o}(X)\right|
$$

where $F_{n}(X)$ is the sample cumulative distribution function (CDF) and $F_{o}(X)$ is the hypothetical CDF. In a normal probability plot $F_{n}(X)$ is the $z$-score value at estimated effect $X$ and $F_{o}(X)$ is the corresponding predicted value on the straight fit line at $X$. The value of $D_{n, \alpha}$ is then compared with the table values. When $D_{n, \alpha}$ is smaller than predicted, then the linear fit line can be accepted as a representative of the data distribution.

\section{A.2.2 Lenth's plot}

Experiments in factorial analysis are sometimes replicated to reduce statistical errors. For unreplicated experiments, a plot corresponding to the results of the method developed by Lenth [85] can supplement the normal probability plot in analysis. In general $2^{k}$ factorial for a group of estimated effects $c_{j}(j=1 . . k)$ value so is defined such as

$$
s_{o}=1.5 \times \operatorname{median}_{j}\left|c_{j}\right|,
$$


and the pseudo standard error (PSE) is defined as

$$
P S E=1.5 \times \operatorname{median}_{\left|c_{j}\right|<2.5 s_{o}}\left|c_{j}\right| .
$$

Margin of error $(M E)$ for $c_{j}$ with approximately $95 \%$ confidence is defined as

$$
M E=t_{0.975 d} \times P S E
$$

where $t_{0.975 d}$ is the 0.975 th quantile of a $t$-distribution on $d=\frac{2 k-1}{3}$ degrees of freedom. To account for the possibility of inferences, a simultaneous margin of error ( $S M E)$ is also defined:

$$
S M E=t_{\gamma, d} \times P S E
$$

where

$$
\gamma=0.5\left(1+0.95^{\frac{1}{2 k-1}}\right)
$$

The Lenth's plot is a bar graph showing the contrasts with ś ME and ś SME reference lines added. The bar of contrast that is beyond either SME line is an active ("real") effect. The contrast that is not beyond either ME line is inactive. The bar of a contrast that is in between SME and ME lines in either half of the graph may or may not be active and can be referred back to the results in the normal probability plot. 


\section{Bibliography}

[1] M. Berger, J. Hubbell, S. Seltzer, J. Chang, J. Coursey, R. Sukumar, D. Zucker, and K. Olsen, "XCOM: Photon Cross Sections Database: NIST Standard Reference Database 8 (XGAM)," November 2010.

[2] R. Minder, G. Majni, C. Canali, G. Ottaviani, R. Stuck, J. Ponpon, C. Schwab, and P. Siffert, "Measurement of the drift velocity of charge carriers in mercuric iodide," $J$. Appl. Phys., vol. 45, pp. $5074-6,1974$.

[3] J. Llacer, M. Watt, M. Schieber, R. Carlston, and W. Schnepple, "Preliminary studies of charge carrier transport in mercuric iodide radiation detectors," IEEE T. Nucl. Sci., vol. 21, pp. $305-14,1974$.

[4] G. Ottaviani, C. Canali, and A. A. Quaranta, "Charge carrier transport properties on semiconductor materials suitable for nuclear radiation detectors," IEEE T. Nucl. Sci., vol. 22, pp. 192-204, 1975.

[5] S. Gits and A. Authier, "Plastic defects in $\alpha-\mathrm{HgI}_{2}$ single crystals," J. Cryst. Growth., vol. 58, pp. 473-485, 1982.

[6] F. Milstein, B. Farber, K. Kim, L. Van Den Berg, and W. Schnepple, "Influence of temperature upon dislocation mobility and elastic limit of single crystal $\mathrm{HgI}_{2}$ " Nucl. Instrum. Methods, vol. 213, pp. 65-76, 1983.

[7] G. Georgeson and F. Milstein, "Theoretical study of the dislocation structure in $\mathrm{HgI}_{2}$," Nucl. Instrum. Meth. A, vol. 285, pp. 488-499, 1989. 
[8] J. Ponpon, R. Stuck, P. Siffert, and C. Schwab, "Preliminary results on mercuric iodide nuclear radiation detectors," Nucl. Instrum. Methods, vol. 119, pp. 197-8, 1974.

[9] J. Ponpon, R. Stuck, P. Siffert, B. Meyer, and C. Schwab, "Preliminary results on mercuric iodide nuclear radiation detectors," IEEE T. Nucl. Sci., vol. 22, pp. 182 - 91, 1975.

[10] M. Slapa, G. Huth, W. Seibt, M. Schieber, and P. Randtke, "Capabilities of mercuric iodide as a room temperature x-ray detector," IEEE T. Nucl. Sci., vol. 23, pp. $102-$ $11,1976$.

[11] A. Holzer and M. Schieber, "Reduction of polarization in mercuric iodide nuclear radiation detection," IEEE T. Nucl. Sci., vol. 27, pp. 266-271, 1980.

[12] V. Gerrish, "Polarization and gain in mercuric iodide gamma-ray spectrometers," Nucl. Instrum. Meth. A, vol. 322, pp. 402-413, 1992.

[13] D. McGregor and R. Rojeski, "High-resolution ionization detector and array of such detectors," US Patent No. 6,175,120, January 2001.

[14] D. McGregor, "Collimated radiation detector assembly, array of collimated radiation detectors and collimated radiation detector module," US Patent No. 6,781,132, August 2004.

[15] W. J. McNeil, D. S. McGregor, A. E. Bolotnikov, G. W. Wright, and R. B. James, "Single-charge-carrier-type sensing with an insulated Frisch ring CdZnTe semiconductor radiation detector," Appl. Phys. Lett., vol. 84, pp. 1988-90, 2004.

[16] D. S. McGregor, A. Kargar, A. M. Jones, W. J. McNeil, and M. J. Harrison, "CdZnTe Frisch collar detectors for $\gamma$-ray spectroscopy," Nucl. Instrum. Meth. A, vol. 558, pp. 497-503, 2006.

[17] A. Kargar, A. M. Jones, W. J. McNeil, M. J. Harrison, and D. S. McGregor, “CdZnTe 
Frisch collar detectors for $\gamma$-ray spectroscopy," Nucl. Instrum. Meth. A, vol. 558, pp. $497-503,2006$.

[18] A. Kargar, A. Brooks, M. Harrison, H. Chen, S. Awadalla, G. Bindley, and D. McGregor, "Effect of crystal length on CdZnTe Frisch collar device performance," in IEEE Nucl. Sci. Conf. R., pp. 2017 - 22, 2009.

[19] A. Kargar, R. B. Lowell, M. J. Harrison, and D. S. McGregor, "The crystal geometry and the aspect ratio effects on spectral performance of CdZnTe Frisch collar device," in Proceedings of SPIE - The International Society for Optical Engineering, vol. 6706, p. 67061J, 2007.

[20] I. Nicolau, "Solution growth of sparingly soluble single-crystals from soluble complexes. I. General introduction," J. Cryst. Growth, vol. 48, pp. 45-50, 1980.

[21] I. F. Nicolau, "Solution growth of sparingly soluble single-crystals from soluble complexes. 2. Growth of alpha- $\mathrm{HgI}_{2}$ single-crystals from iodomercurate complexes," $J$. Cryst. Growth, vol. 48, pp. 51-60, 1980.

[22] I. F. Nicolau and J. P. Joly, "Solution growth of sparingly soluble single-crystals from soluble complexes. iii. Growth of alpha- $\mathrm{HgI}_{2}$ single-crystals from dimethylsulfoxide complexes," J. Cryst. Growth, vol. 48, pp. 61-73, 1980.

[23] M. Schieber, R. Carlston, H. Lamonds, P. Randtke, F. Schnepple, and J. Llacer, "Purification, growth, and characterization of alpha mercuric-iodide crystals for gammaray detection," Nucl. Instrum. Methods, vol. 24-25, pp. 205-11, 1974.

[24] M. Schieber, W. Schnepple, and L. Van Den Berg, "Vapor growth of $\mathrm{HgI}_{2}$ by periodic source or crystal temperature oscillation," J. Cryst. Growth, vol. 33, no. 1, pp. 125 35, 1976.

[25] H. Lamonds, "Review of mercuric iodide development program in Santa Barbara," Nucl. Instrum. Methods, vol. 213, pp. 5-12, 1983. 
[26] H. Scholz, "On crystallization by temperature-gradient reversal," Acta Electron., vol. 17 , no. 1 , pp. $69-74,1974$.

[27] A. E. Bolotnikov, J. Baker, R. DeVito, J. Sandoval, and L. Szurbart, " $\mathrm{HgI}_{2}$ detector with a virtual Frisch ring," IEEE T. Nucl. Sci., vol. 52, pp. 468-472, 2005.

[28] S. Faile, A. Dabrowski, G. Huth, and J. Iwanczyk, "Mercuric iodide $\left(\mathrm{HgI}_{2}\right)$ platelets for x-ray spectroscopy produced by polymer controlled growth,” J. Cryst. Growth, vol. 50, no. 3, pp. 752-756, 1980.

[29] A. Burger, M. Roth, and M. Schieber, "The role of polyethylene in platelet growth of mercuric iodide," Journal of Crystal Growth, vol. 56, pp. 526 - 531, 1982.

[30] A. Burger, A. Levi, J. Nissenbaum, M. Roth, and M. Schieber, "Yield and quality of $\mathrm{HgI}_{2}$ vapor grown platelets," J. Cryst. Growth, vol. 72, pp. 643-648, 1985.

[31] J. Laskowski, H. Kuc, K. Conder, and J. Przyłuski, "Growth of $\alpha-\mathrm{HgI}_{2}$ platelets," Mater. Res. Bull., vol. 22, pp. 715-22, 1987.

[32] J. Przyłuski and J. Laskowski, " $\alpha-\mathrm{HgI}_{2}$ platelets technology,” Nucl. Instrum. Meth. A), vol. A283, pp. 144-8, 1989.

[33] B. J. Aylett, The Chemistry of ZINC, CADMIUM, and MERCURY, vol. 18 of Pergamon Texts in Inorganic Chemistry. Pergamon Press, 1973.

[34] N. Lyakh, "Composition and kinetic characteristics of vapour phase during mercuric iodide growing," Crystal Res. Technol., vol. 20, pp. 313-319, 1985.

[35] J. M. Bijvoet, A. Claasen, and A. Karssen, "Crystal structure of red mercuric iodide," Proc. Acad. Sci. Amst., vol. 29, pp. 529-546, 1926.

[36] G. A. Jeffrey and M. Vlasse, "On crystal structures of red yellow and orange forms of mercuric iodide," Inorganic Chemistry, vol. 6, no. 2, pp. 396-399, 1967. 
[37] M. Hostettler, H. Birkedal, and D. Schwarzenbach, "The yellow polymorphs of mercuric iodide $\left(\mathrm{HgI}_{2}\right)$," Helvetica Chimica Acta, vol. 86, no. 5, pp. 1410-1422, 2003.

[38] M. Hostettler and D. Schwarzenbach, "Phase diagrams and structures of $\operatorname{HgX}_{2}(\mathrm{X}=$ I, Br, Cl, F),” C. R. Chim., vol. 8, pp. 147-156, FEB 2005.

[39] J. B. Newkirk, "A crystallographic study of mercuric iodide," Acta Metal., vol. 4, pp. 316-330, 1956.

[40] M. Zha, M. Piechotka, and E. Kaldis, "Vapour growth of bulk anisotropic crystals: case study of $\alpha-\mathrm{HgI}_{2}$, , J. Cryst. Growth, vol. 115, pp. $45-51,1991$.

[41] J. Przyłuski, J. Laskowski, and M. Piechotka, "Vapor growth of $\mathrm{HgI}_{2}$ crystals in presence of organic additives," Materials Research Bulletin, vol. 19, pp. 189-96, 1984.

[42] A. Kargar, E. Ariesanti, and D. S. McGregor, "Characterization of charge collection efficiency for $\mathrm{HgI}_{2}$ spectrometer with collimated high energy gamma-rays," Nucl. Instrum. Methods A, pp. available online, http://dx.doi.org/10.1016/j.nima.2010.08.057, 2010.

[43] E. Ariesanti, A. K. Kargar, and D. S. McGregor, "Mercuric iodide crystal growth and Frisch collar detector fabrication," Nucl. Instrum. Meth. A., vol. 624, pp. 656 - 661, 2010.

[44] P. Ugorowski, E. Ariesanti, D. S. McGregor, and A. Kargar, "Compact, inexpensive, high-energy-resolution, room-temperature-operated, semiconductor gamma-ray detectors for isotope identification," in Conference Record of the IEEE International Conference on Technologies for Homeland Security, pp. 385 - 390, 2010.

[45] E. Ariesanti, A. Kargar, and D. S. McGregor, "Mercuric iodide crystal growth and Frisch collar detector fabrication," Nucl. Tech., vol. 175, pp. 124 - 130, 2011.

[46] A. Kargar, E. Ariesanti, and D. S. McGregor, "A comparison between spectroscopic 
performance of $\mathrm{HgI}_{2}$ and CdZnTe Frisch collar detectors," Nucl. Tech., vol. 175, pp. $131-137,2011$.

[47] S. P. Faile, "Vapor growth of mercuric iodide for use as high energy detectors," US Patent No. 4,282,057, August 1981.

[48] J. Laskowski, H. Kuc, K. Conder, and J. Przyłuski, "Growth of $\alpha-\mathrm{HgI}_{2}$ platelets," Cryst. Res. Technol., vol. 23, pp. 1231 - 1237, 1988.

[49] J. Laskowski, K. Conder, and J. Przyłuski, "Diffusive-convective mass transport of $\mathrm{HgI}_{2}$ in closed ampoules," Cryst. Res. Technol., vol. 23, pp. 1239 - 1244, 1988.

[50] K. Conder and J. Laskowski, "The vapour transport mechanism during crystallization of $\mathrm{HgI}_{2}$ in closed ampoules," Nucl. Instrum. Methods A, vol. 283, pp. 138 - 143, 1989.

[51] J. Laskowski, J. Olędzka, and J. Przyłuski, "Residual gases in $\mathrm{HgI}_{2}$ crystal growth ampoules," J. Mater. Sci., vol. 25, pp. 589 - 594, 1990.

[52] J. D. Phillips, M. W. Lund, J. K. Shurtleff, and W. P. Allred, "Advancements in platelet growth," Nucl. Instrum. Meth. A., vol. 380, pp. 50 - 52, 1996.

[53] E. Ariesanti, C. Frampton, S. Appelhans, M. Rudolph, and D. McGregor, "A systematic study of mercuric iodide platelet growth in horizontal furnaces," vol. 7079, pp. 70790L-70797L, 2008.

[54] E. Ariesanti, D. S. McGregor, and A. Kargar, "Vapor growth of tetragonal prismatic mercuric iodide crystals," in Conference Record of the IEEE Nuclear Science Symposium and Medical Imaging Conference and 17th International Workshop on RoomTemperature Semiconductor X-ray and Gamma-ray Detectors, pp. 3739 - 3745, 2010.

[55] H. E. Buckley, Crystal Growth. John Wiley and Sons, Inc., 1st ed., 1951.

[56] E. Ariesanti and D. S. McGregor J. Cryst. Growth, 2011. 
[57] T. Hahn, U. Shmueli, and A. Wilson, eds., Space-group symmetry, vol. A of International Tables for Crystallography. International Union of Crystallography, 5th ed., 2005.

[58] L. Finger, M. Kroeker, and B. Toby, "DRAWxtl, an open-source computer program to produce crystal structure drawings," J. Appl. Crystallogr., vol. 40, pp. 188-192, 2007.

[59] P. Hartman, Crystal Growth: an Introduction. North Holland Publishing Company, 1973.

[60] P. Bennema, H. Meekes, S. X. M. Boerrigter, H. M. Cuppen, M. A. Deij, J. van Eupent, P. Verwer, and E. Vlieg, "Crystal growth and morphology: New developments in an integrated Hartman-Perdok-connected net-roughening transition theory, supported by computer simulations," Cryst. Growth Des., vol. 4, pp. 905-913, 2004.

[61] Specialty coating systems, Model PDS 2010 LABCOTER 2 Operator ̌̌s Manual, 2006.

[62] P. S. Ho, J. Leu, and W. W. Lee, Low dielectric constant materials for IC applications. New York: Springer, 1st ed., 2003.

[63] A. Kargar, A. C. Brooks, M. J. Harrison, K. T. Kohman, R. B. Lowell, R. C. Keyes, H. Chen, G. Bindley, and D. S. McGregor, "The effect of the dielectric layer thickness on spectral performance of CdZnTe Frisch collar gamma ray spectrometers," IEEE T. Nucl. Sci., vol. 56, pp. 824-831, 2009.

[64] C. Guminski, “The Hg-I (mercury-iodine) system,” J. Phase Equi., vol. 1B, pp. 206$215,1997$.

[65] E. Benham and M. McDaniel, Polyethylene, High Density. Kirk-Othmer Encyclopedia of Chemical Technology, 2005.

[66] M. Broadhurst, "Extrapolation of the orthorhombic n-paraffin melting properties to very long chain lengths," J. Chem. Phys., vol. 36, pp. 2578-2582, 1962. 
[67] C. Leray, Waxes. Kirk-Othmer Encyclopedia of Chemical Technology, 2005.

[68] J. C. Salamone, Polymeric materials encyclopedia, vol. 10, p. 3616. CRC Press, 1996.

[69] F. C. Phillips, An introduction to crystallography. Wiley, 4th ed., 1971.

[70] W. Kaminsky, “WinXMorph: a computer program to draw crystal morphology, growth sectors and cross sections with export files in VRML V2.0-virtual reality format," $J$. Appl. Cryst., vol. 38, pp. 566-567, 2005.

[71] W. Kaminsky, "From CIF to virtual morphology using the WinXMorph program," J. Appl. Cryst., vol. 40, pp. 382 - 5, 2007.

[72] N. W. Ashcroft and N. D. Mermin, Solid State Physics. Holt, Rinehart and Winston, 1976.

[73] B. Cullity, Elements of X-Ray Diffraction. Addison-Wesley, 1956.

[74] B. V. Novikov and M. M. Pimonenk, "Exciton absorption and luminescence of tetragonal $\mathrm{HgI}_{2}$ at low temperatures," Sov. Phys. Semicond., vol. 4, pp. 1785 -, 1971.

[75] B. V. Novikov and M. M. Pimonenk, "Influence of temperature on excited luminescence of tetragonal $\mathrm{HgI}_{2}$ single-crystals," Sov. Phys. Semicond., vol. 6, pp. 671 -, 1972.

[76] A. Burger and D. Nason, "Optical determinations of the direct energy-gap in alphamercuric iodide at elevated temperatures," J. Appl. Phys., vol. 71, pp. 2717 - 2720, 1992.

[77] D. Wong, T. E. Schlesinger, R. B. James, C. Ortale, L. van den Berg, and W. F. Schnepple, " $\mathrm{HgI}_{2}$ near-band-gap photoluminescence structure and its relationship to nuclear detector quality," J. Appl. Phys., vol. 64, pp. 2049 - 2054, 1988.

[78] D. Wong, X. J. Bao, T. E. Schlesinger, R. B. James, A. Cheng, C. Ortale, and L. van den Berg, "Photoluminescence variations associated with the deposition of 
palladium electrical contacts on detector-grade mercuric iodide," Appl. Phys. Lett., vol. 53, pp. $1536-1538,1988$.

[79] Z. L. Wu, J. L. Merz, L. van den Berg, and W. F. Schnepple, "Low temperature photoluminescence of $\mathrm{HgI}_{2}$," J. Lumin., vol. 24 - 25, pp. 197 - 200, 1981.

[80] J. L. Merz, Z. L. Wu, L. van den Berg, and W. F. Schnepple, "Low temperature photoluminescence of detector-grade $\mathrm{HgI}_{2}$," Nucl. Instrum. Methods, vol. 213, pp. 51 $-64,1983$.

[81] R. B. James, X. J. Bao, T. E. Schlesinger, A. Y. Cheng, C. Ortale, and L. van den Berg, "Photoluminescence investigations of defects inroduced during processing of mercuric iodide nuclear detectors," Nucl. Instrum. Methods A, vol. 322, pp. 435 441, 1992.

[82] G. Box, W. Hunter, and J. Hunter, Statistics for experimenters: an introduction to design, data analysis, and building. Wiley, 5th ed., 1978.

[83] D. T. Mage, "An objective graphical method for testing normal distributional assumptions using probability plots," Am. Stat., vol. 36, pp. 116-120, 1982.

[84] H. W. Lilliefors, "On the Kolmogorov-Smirnov test for normality with mean and variance unknown," J. Am. Stat. Assoc., vol. 62, pp. 399-402, 1967.

[85] R. V. Lenth, "Quick and easy analysis of unreplicated factorials," Technometrics, vol. 31, pp. 469-473, 1989. 